Characteristics and recognizability of vocal expressions of emotion 

CHARACTERISTICS AND RECOGNIZABILITY

OF VOCAL EXPRESSIONS OF EMOTION 



\section{Characteristics and recognizability of vocal expressions of emotion}

Proefschrift

ter verkrijging van de graad van doctor in de letteren

aan de Katholieke Universiteit te Nijmegen, op gezag van de Rector Magnificus

Prof. Dr. J.H.G.I. Giesbers

volgens besluit van het College van Dekanen

in het openbaar te verdedigen

op 4 oktober 1984 des namiddags te 2.00 uur precies

door

RENEE ALICE MARTHA GOVERINA VAN BEZOOIJEN geboren te Kortrijk, Belgie

FORIS PUBLICATIONS

Dordrecht - Holland/Cinnaminson - U.S.A. 
Promotores: Prof. Dr. W.H. Vieregge

Prof. Dr. K.R. Scherer 


\section{CONTENTS}

1. INTRODUCTION

1.1 Introduction 1

1.2 Description 2

1.3 Recognition 3

1.4 Categorical and dimensional approach 4

1.5 Structure of the book 5

2. SPEECH MATERIAL 7

2.1 Introduction 7

2.2 Method 13

2.2.1 Speakers 13

2.2.2 Phrases 13

2.2.3 Instructions 14

2.2.4 Recordings 14

3. PERCEPTUAL CHARACTERISTICS OF DISCRETE EMOTIONS $\quad 17$

3.1 Introduction 17

$\begin{array}{ll}3.2 \text { Method } & 19\end{array}$

3.2.1 Speech material 19

$\begin{array}{ll}3.2 .2 \text { Raters } & 19\end{array}$

3.2.3 Parameters 19

3.2.4 Rating 21

3.3 Results 21

3.3.1 Reliability 22

3.3.2 Factors sex of speaker, phrase, and emotion 22

3.3.2.1 Factor sex of speaker $\quad 22$

3.3.2.2 Factor phrase $\quad 24$

3.3.2.3 Factor emotion $\quad 24$

$\begin{array}{ll}3.4 \text { Discussion } & 28\end{array}$

3.4.1 Reliability 28

3.4.2 Factor sex of speaker $\quad 29$

$\begin{array}{ll}\text { 3.4.3 Factor phrase } & 30\end{array}$

3.4.4 Perceptual characteristics of emotions 31 
4. RELATIVE IMPORTANCE OF PERCEPTUAL PARAMETERS AND THE CHARACTERISTICS OF EMOTIONAL DIMENSIONS

4.1 Introduction $\quad 39$

4.2 Method 42

4.2.1 Input data $\quad 42$

4.2.2 Analysis $\quad 42$

4.3 Results 43

4.3.1 Classification 43

4.3.2 Dimensions 46

4.3.3 Confusions 49

4.4 Discussion 51

4.4.1 Classification 51

4.4.2 Relative importance of perceptual parameters $\quad 52$

4.4.3 Dimensions 53

5. ACOUSTIC CORRELATES OF PERCEPTUAL PARAMETERS 57

5.1 Introduction $\quad 57$

5.2 Method 58

5.2.1 Perceptual ratings $\quad 58$

5.2.2 Measures of tempo 58

5.2.3 The measurement of fundamental frequency 59

5.2.3.1 Measures of central tendency of $F_{0} \quad 59$

5.2.3.2 Measures of $F_{0}$ variation $\quad 60$

5.2.3.3 Measures of $F_{0}$ perturbations $\quad 60$

5.2.4 Measures of spectral slope 61

5.3 Results 64

5.3.1 Ratings of tempo related to syllable duration 64

5.3.2 Ratings of pitch level explained by measures of central tendency of $F_{0}$

5.3.3 Ratings of pitch range explained by measures of $F_{0}$ variation 65

5.3.4 Ratings of harshness explained by measures of $F_{0}$ perturbations

5.3.5 Ratings of loudness, tension, and laxness explained by measures of spectral slope

5.3.6 A multiple regression approach $\quad 67$

5.3.7 Discriminant analysis 69

5.4 Discussion 70

6. RECOGNITION BY DUTCH ADULTS 75

6.1 Introduction $\quad 75$

6.2 Method 83

6.2.1 Subjects $\quad 83$

6.2.2 Speech material 83 
$\begin{array}{ll}\text { 6.2.3 Procedure } & 83\end{array}$

6.3 Results 84

6.3.1 Factor emotion $\quad 84$

6.3.2 Factors sex of speaker and sex of subject 86

6.3.3 Factor phrase $\quad 86$

$\begin{array}{ll}6.4 \text { Discussion } & 87\end{array}$

6.4.1 Factor emotion $\quad 87$

6.4.2 Factors sex of speaker and sex of subject 89

$\begin{array}{ll}\text { 6.4.3 Factor phrase } & 90\end{array}$

7. DEVELOPMENTAL RECOGNITION 91

7.1 Introduction 91

$\begin{array}{ll}7.2 \text { Method } & 96\end{array}$

7.2.1 Subjects $\quad 96$

7.2.2 Speech material $\quad 97$

$\begin{array}{ll}7.2 .3 \text { Instruments } & 98\end{array}$

7.2.4 Response categories $\quad 98$

$\begin{array}{ll}7.2 .5 \text { Procedure } & 100\end{array}$

$\begin{array}{ll}7.3 \text { Results } & 102\end{array}$

$\begin{array}{ll}\text { 7.3.1 Factor age } & 102\end{array}$

$\begin{array}{ll}\text { 7.3.2 Factor sex of subject } & 104\end{array}$

$\begin{array}{ll}\text { 7.3.3 Factor test } & 104\end{array}$

7.3.4 Confusions 105

$\begin{array}{ll}7.4 \text { Discussion } & 106\end{array}$

7.4.1 Factor age 106

$\begin{array}{ll}\text { 7.4.2 Factor sex of subject } & 108\end{array}$

7.4.3 Factor test 109

$\begin{array}{ll}\text { 7.4.4 Confusions } & 112\end{array}$

8. RECOGNITION BY TODDLERS 113

8.1 Introduction 113

8.2 Method 114

8.2.1 Subjects 114

8.2.2 Speech material $\quad 114$

8.2.3 Instruments 115

8.2.4 Response categories 115

8.2.5 Procedure 115

$\begin{array}{lll}8.3 \text { Results } & 117\end{array}$

$\begin{array}{ll}8.4 \text { Discussion } & 119\end{array}$

9. RECOGNITION BY DUTCH, TAIWANESE, AND JAPANESE SUBJECTS

9.1 Introduction 121

$\begin{array}{ll}9.2 \text { Method } & 124\end{array}$

9.2.1 Subjects 124 
9.2.2 Speech material $\quad 124$

9.2.3 Procedure 124

9.3 Results 125

9.3.1 Rate of recognition $\quad 126$

$\begin{array}{ll}\text { 9.3.2 Confusions } & 129\end{array}$

9.4 Discussion 137

10.INTEGRATION OF FINDINGS AND SUGGESTIONS FOR FURTHER RESEARCH 141

10.1 Introduction 141

10.2 Cross-cultural similarity of discrete emotions 141

10.3 Emotional dimensions $\quad 142$

10.4 Rate of recognition 145

10.5 Some methodological remarks and suggestions for further research

APPENDIX A. Acoustic data for ten emotions

APPENDIX B. Instructions for the recognition experiment with Dutch adults

APPENDIX C. Combinations and order of the alternatives in the developmental recognition experiment

APPENDIX D. Combinations and order of the alternatives in the recognition experiment with toddlers

REFERENCES

SAMENVATTING 


\section{Acknowledgements}

The research reported on in this thesis was funded by the Research Pool of the Catholic University of Nijmegen on the basis of a three year contract; it was carried out at the Institute of Phonetics of this University.

I would like to thank my student-assistents Wim Hoogeveen for the time he saved me in carrying out those countless little jobs that research involves and Annie van den Oever for her ever present, stimulating interest and the devotion with which she handled each activity she undertook.

I would further like to thank Hans Zondag, who made the recordings, Stan Otto and Tom Heenan, who conducted the cross-cultural experiments, the pupils and teachers of "t Stulpje" and "de Piekenstulp", and all the other children and adults who in one way or another participated in this research, either as subjects, speakers, or raters.

Finally, I wish to express my gratitude to Joe for going so patiently and carefully over my English and to the others who read through the manuscript but whose names I cannot mention for various reasons. 



\section{Introduction}

\subsection{INTRODUCTION}

Any study on emotion is expected to begin by defining what is meant by the term. However, when consulting the literature one gets the impression that there are almost as many definitions as there are studies (for an overview of definitions presented, see Plutchik, 1980, p. 81-83). As Strongman (1978) puts it, "there is no consensus of opinion; at present emotion defies definition" (p. 1-2).

Nevertheless, it is our common every-day experience that emotions such as anger, fear, and joy exist. We have all experienced them when confronted with particular objects or events, either directly in the here and now, by recalling them in the past, or by envisaging them in the future, and we have a fairly clear idea of what feelings labels such as anger, fear, and joy refer to. We also know that emotional experiences are not purely private, but that they have outward effects. We make use of these manifestations in interpreting other people's behaviour, just as they make use of them in interpreting ours. The expressive component of emotional experiences is an important aid in guiding social interaction.

Much research has been conducted into emotional expressions. However, not all channels have received equal attention. The face has been studied most extensively (see Davitz, 1964a; Ekman, Friesen, \& Ellsworth, 1972; Izard, 1971 for reviews); posture and gestures have hardly been looked at; and the voice takes an intermediate position. It is the last aspect of emotional expression that is the object of the present study. The aim of this study was to establish the characteristics of vocal expressions of emotion in Dutch and their recognizability as a function of sex, age, and culture.

The empirical study of vocal expressions of emotion - "vocal" referring to "how" things are said as opposed to "what" is being said, or, phrased differently, "vocal" referring to everything that remains present in a spoken message after lexical and syntactic information has been removed - may be traced back to the twenties, but it was not until the sixties that it really got started. A major landmark is the year 1964 when Davitz, a clinical psychologist wondering "how to go about being 'sensitive, empathic, and emotionally 
understanding"' (1964d, p. 2), published a collection of studies the variety and orientation of which is a good reflection of the kind of research that was to follow. In addition to a review of research into vocal expressions of emotion (Davitz, 1964a), the collection includes samples of descriptive research, which aims at finding the perceptual or acoustic characteristics of vocal expressions of emotion (Davitz, 1964c), of correlational research, which attempts at assessing, for example, the relationship between the abilities to express and perceive vocal expressions of emotion (Levy, 1964), and of recognition research, investigating the recognizability of vocal expressions of emotion and the way in which it is affected by various variables, such as age and sex (Dimitrovsky, 1964). In the present work the correlational area of research into vocal expressions of emotion will not be dealt with. Both the description and recognition areas, however, are covered by a number of studies.

\subsection{DESCRIPTION}

In the first part of this book, comprising Chapters 3, 4, and 5, a description is presented which aims at establishing the vocal characteristics of emotions in Dutch. The first two of these chapters deal with perceptual ratings, the last one with acoustic measures.

Although quite a few studies have been conducted which aimed at obtaining perceptual or acoustic descriptions of vocal expressions of emotion, it appears that the knowledge gathered to date is rather limited (for an overview of descriptive studies and their experimental setup, see Scherer (1981b) and Table 3.7 in the present work). The majority of studies have been based on American English expressions, and only a few emotions, namely joy, fear, sadness, and anger, and a few vocal parameters, namely pitch level, pitch range, loudness, and tempo, have been examined more or less systematically. In many cases descriptions have been made of the emotional utterances produced by one or two speakers. The relationships among the vocal parameters and their relative importance for the discrimination among emotions have rarely been considered.

In the present work, in addition to the characteristics of joy, fear, sadness, and anger, those of five other emotions as well as a neutral category were assessed. The expressions have been produced by eight Dutch adults, four males and four females, with two different phrases. Just like in most other studies to date, the parameters of pitch level, pitch range, loudness, and tempo were considered. This inventory was extended, however, to include the perceptual ratings of lip rounding, lip spreading, laryngeal tension, laryngeal laxness, creak, tremulousness, whisper, harshness, and precision of articulation, and the acoustic measurements of laryngeal tension, laryngeal laxness, and harshness. The meaning of the parameters has been carefully defined in order to allow replication of the experiments and meaningful 
comparisons of the outcomes. The reliability of the ratings has also been determined. The work reported here contributes to the development of a comprehensive system for the phonetic description of non-verbal aspects of speech. The lack of such a tool has greatly hampered research into vocal expressions of emotion.

A number of statistical and data processing techniques have been applied in order to test various effects and gain insight into the structure of the descriptive data. By doing so we were able, for example, to assess which parameters varied as a function of the emotions, the sex of the speakers, and the phrase which was used as the carrier for the expression of the emotions, to examine the relationships among the parameters, and to gain insight into the relevance of various parameters for the discrimination among the emotions expressed.

\subsection{RECOGNITION}

In the second part of this book, comprising Chapters 6, 7, 8, and 9, it is assessed how well the various emotions can be recognized as a function of sex, age, and cultural distance between speaker and subject. The subject groups comprise Dutch female and male adults, Dutch boys and girls of varying ages, and Japanese and Taiwanese adults.

The recognition of vocal expressions of emotions by adults has been studied quite extensively (see Table 6.1 of the present work for a review). Almost all research to date, however, has dealt with American English expressions. The recognition of vocal expressions of emotions by children has received considerably less attention (see Table 7.1 of the present work for a review). Again, the developmental research to date has almost exclusively been concerned with the recognizability of American English expressions. Moreover, only a few emotions, especially joy and anger, have been examined systematically. Also, the performance of children below age 7 has hardly been looked at. In the present work, the recognizability of Dutch expressions of nine emotions has been assessed for children between age 5 and 11 , and that of six emotions for children age 3 . The results of these experiments provide new information on the way in which the ability to recognize emotions develops from early childhood on.

The cross-cultural recognition experiments are of importance because, unlike information on the cross-cultural recognition of facial expressions of emotion, data on the cross-cultural recognizability of vocal expressions of emotion are scarce. Such data - combined with detailed descriptions of expressions produced by speakers from different cultures - are essential for determining to what extent the vocal characteristics of emotions are culture specific and to what extent they are cross-culturally, and possibly even universally, similar. This question is one of the main issues - and perhaps the most intriguing one - of research into emotional expression. 
In all recognition studies reported special attention has been paid to the question of which emotions are confused with one another. The outcomes of these analyses add to our knowledge of the relationships among emotions, which ones are perceptually similar and which ones are perceptually dissimilar, and the extent to which the perceived structures are shared by subjects of different ages and varying cultural backgrounds.

\subsection{CATEGORICAL AND DIMENSIONAL APPROACH}

At the base of the present work are the vocal expressions of nine emotional categories, namely, disgust, surprise, shame, interest, joy, fear, contempt, sadness, and anger, supplemented in most cases with a neutral category. These nine emotions are considered by major emotion theorists such as Izard (1971, 1977) and Tomkins (1962) to be "primary" (also "prototypical", "fundamental", "basic"). By this they mean that they consider these emotions to be discrete categories characterized by specific facial patterns which are genetically determined and triggered by built-in neural programs. Evidence in favour of this approach has been drawn from recognition research in which it was shown that people from widely differing cultures are able to keep the facial expressions of most of these emotions apart (cf. Ekman, 1973; Izard, 1977). (Of course, although it is shown that the facial patterns that characterize particular primary emotions are similar across cultures, no one denies that the stimuli by which particular emotions are provoked may vary from culture to culture, and that there are culture specific norms and conventions as to when and how particular emotions are displayed.)

The above view of the structure of emotions in terms of a small number of specific emotions which are discretely different from one another and a great number of others which are second or higher order mixtures, is generally referred to as the categorical approach to the study of emotion, and goes back to and has been heavily influenced by the ideas developed by Darwin in "The expression of the emotions in man and animals" (1872). Another angle from which emotions have been considered (e.g. Schlosberg, 1954), is the so-called dimensional approach, in which emotions are assumed to be characterized by gradual and fluent transitions, and in which differences and similarities among emotions are described in terms of distances along dimensions that run, for example, from passive to active or from negative (unpleasant) to positive (pleasant). The two approaches are not mutually exclusive. In the theory developed by Plutchik (1980), for example, and in the "hierarchical" model proposed by Frijda (1970) the concepts of discrete emotions and dimensionality have been integrated. According to Dittman (1972), the two approaches differ mainly in that the studies "from the category viewpoint tell us how high one's accuracy can be under ideal circumstances, and may thus be classified as studies about competence. The dimension studies tell us more about how the person approaches an expression, and what sorts 
of things he pays attention to first - such as whether it is pleasant or unpleasant, and so on down the line - and may thus be classified as performance studies" (p. 73).

In the present study elements of the categorical and dimensional approaches have been combined. Typical of the categorical approach is the fact that in the recognition experiments the subjects were offered expressions that represented the emotional categories chosen for study in their purest possible form, and that they were asked to respond in terms of these same pure categories. The dimensional approach would typically have led to the use of blends of emotions and responses in terms of judgments on semantic differential scales or in terms of similarities among pairs or triads of expressions.

In line with the dimensional approach is the fact, for example, that in the recognition studies, in addition to assessing the extent to which subjects were able to identify the emotional categories correctly, we have paid attention to the question of whether confusions among emotions can be explained in terms of their location on particular emotional dimensions. Likewise, in the description studies, in addition to determining the perceptual characteristics of the discrete emotional categories under study, we have tried to gain insight into the perceptual characteristics of emotional dimensions by performing a number of discriminant analyses and considering the functions extracted.

\subsection{STRUCTURE OF THE BOOK}

The structure of this book is as follows:

In Chapter 2, a description is given of the speech material which has served as a basis for the studies reported on in the subsequent chapters. Attention is paid to the way in which researchers into the vocal communication of emotions have solved the problem of how to separate the vocal from the verbal channel and to the arguments which have led to our decision to opt for the study of simulated rather than spontaneous emotional expressions.

In Chapter 3, a descriptive study is presented in which half of the material was offered to a small panel of raters with the request to score the expressions on thirteen vocal parameter scales. The aim of this study was to see whether there are specific (combinations of) vocal features that characterize the emotional categories investigated. A methodological point of interest is the reliability of the scoring.

In Chapter 4, a study is reported in which the perceptual data obtained in the study described in Chapter 3 were used as input into a number of discriminant analyses in order to gain insight into the relative importance of the various vocal parameters scored to the - statistical - separation of the ten emotions.

In Chapter 5, a description is given of an attempt to find the best possible (set of) acoustic correlate(s), from a number of plausible candidates, of the 
perceptual scores on seven of the parameters referred to in Chapter 3. Just like the perceptual scores, the acoustic correlates obtained have served as a basis for an analysis of discriminance.

In Chapter 6, it is assessed how well the emotional expressions can be recognized by Dutch adults, and to what extent recognition is affected by the sex of the subject, the sex of the speaker, and the phrase which was used as the carrier for the stimuli.

The studies reported on in Chapters 7 and 8 deal with the recognition by Dutch children. Central in the study described in Chapter 7 are the questions of how recognition develops from age 5 on, whether there is a difference between boys and girls, whether recognition depends on the way in which the subjects were asked to respond, and whether the errors made by children resemble those made by adults. In Chapter 8 , a similar recognition experiment with $3 \frac{1}{2}$-year-old toddlers is described.

In Chapter 9, it is assessed to what extent Dutch, Taiwanese, and Japanese adults differ in their abilities to identify Dutch expressions of emotion and whether there are similarities in the confusions made. The aim of this study is to gain insight into the cross-cultural recognizability of vocal expressions of emotion.

Finally, in Chapter 10, some description and recognition findings obtained in the preceding chapters are integrated, and suggestions are made for further research. 


\section{Speech material}

\subsection{INTRODUCTION}

No doubt most people would agree that the best speech material on which to base the study of both the characteristics and the recognizability of vocally expressed emotions would be speech material produced spontaneously in a real-life setting. Nevertheless, it appears that relatively few empirical studies on vocal expressions of emotion have made use of natural speech, notable exceptions being some studies conducted by Brown (1980), Huttar (1968), and Williams and Stevens $(1972,1981)$.

Brown (1980) carried out a recognition study in which tape recorded speech samples (played backwards so that it was impossible to understand what was being said) were offered, produced by a young couple upon the occasion of the birth of their first child. The recordings had been made at different stages of the event: during a visit to the doctor, in the labour room at delivery, and after delivery. Recognition was assessed by counting the number of times that subjects were able to tell during which of the three stages each speech sample had been uttered. In the same publication Brown mentions ongoing studies in which the voices of athletes before and after winning and losing were used, and recordings of reminiscences of happy, sad, difficult, etc. life experiences.

In a study in which the relationship between emotional meaning and various vocal speech parameters was assessed, Huttar (1968) offered the utterances of one adult male taken from a series of discussions and classroom lectures. Emotional meaning was established by means of ratings on semantic differential scales, the terms of which were descriptive of emotions.

Williams and Stevens (1972) acoustically analyzed the recordings of a radio-announcer describing the approach of the Hindenburg at Lakehurst, New Jersey, when the zeppelin suddenly burst into flames. The announcer continued his description (with one or two short breaks) throughout the disaster. Williams and Stevens (1969, cited in Williams \& Stevens, 1981) also analyzed the recordings of a conversation between a civilian pilot and a control tower operator. During the course of the recording the pilot encountered serious problems with his aircraft. The recording ended with the pilot losing control and crashing. 
There are also a number of studies which have made use of speech samples that, although not obtained in real-life situations, nevertheless contain some natural elements. Brown (1980), for example, carried out a recognition experiment in which descriptions of films varying in emotional impact were offered to a group of subjects who, after having seen the same films, were requested to indicate which film was being described. The descriptions were in Spanish, a language with which none of the subjects were familiar; hence, they could only rely on vocally transmitted information for their choice. A somewhat similar procedure for obtaining stimuli was employed by Markel, Bein, and Phillis (1973). They had subjects describe Thematic Apperception Test (TAT) cards. The descriptions were categorized in terms of the degree of outward directed and inward directed hostility and then related to auditory ratings of pitch, loudness, and tempo.

A common characteristic of the studies mentioned above is that the verbal content of the speech samples is not controlled for. This may be a serious disadvantage. It means, for example, that if one wants to assess the extent to which emotional meaning can be derived from nonverbal properties of speech alone, measures will have to be taken to make sure that possibly relevant information contained in the verbal channel is eliminated. The separation of the verbal channel and the vocal channel has been one of the major problems facing researchers investigating the recognition of vocal expressions of emotion. One solution to this problem has been sought in the application of different content masking techniques, i.e., techniques which render the verbal content of speech unintelligible while preserving most of the vocal information.

A simple content masking technique, which has been used, for example, in one of the above mentioned studies by Brown and in a study by Knower (1941), is to play the tape with the recorded speech backwards. In this way speech is rendered unintelligible, whereas voice quality, tempo, pause distribution, pitch range, and overall pitch level are left undisturbed. On the other hand, the technique has the drawback of distorting the original intonation contour, thus removing possible cues to emotional meaning.

Another content masking technique is random splicing, a technique developed by Scherer (1971) and utilized in a number of subsequent studies (e.g. Blanck, Rosenthal, Snodgrass, DePaulo, \& Zuckerman, 1981; Scherer, Koivumaki, \& Rosenthal, 1972). Basically, this technique consists of removing the pauses from a stretch of recorded speech, cutting the rest of the tape into pieces, and splicing these back together in random order (at first this was done by hand, later electronically). If the pieces are short enough (between 150 and $300 \mathrm{~ms}$ ), this procedure results in almost total unintelligibility even after repeated exposure, whereas voice quality, pitch, and amplitude, both in terms of average level and range, as well as rate are preserved. As a content masking device, it has the same drawback as the preceding technique, namely 
of removing various cues which may be relevant to the communication of emotional meaning, such as temporal features and intonation patterns. But then, it is exactly the fact that certain cues are no longer present which makes it possible to use this type of technique in recognition studies to gain insight into the contribution of different cues to the transmission of emotional meaning (see also Section 4.1).

A third way in which content may be masked is to pass the recorded speech through an electronic lowpass filter. In this way high frequencies, which play an important role in the distinction of speech sounds and are hence crucial to speech comprehension, are removed (for a detailed description of an electronic filtering system, see Rogers, Scherer, \& Rosenthal, 1971). Unfortunately, the higher frequencies also contribute to voice quality perception, which means that this technique too may result in the loss of emotionally relevant information. Moreover, it appears that if the same speech sample is repeated of ten enough, even at fairly low cut-off frequencies $(400-450 \mathrm{~Hz})$ subjects end up understanding what is being said (Kramer, 1964; Ross, Duffy, Cooker, \& Sargeant, 1973). Studies in which lowpass filtering has been used are Kramer (1964), Burns \& Beier (1973), Fechner (1978), McCluskey, Niemi, \& Albas (1978), Scherer, Koivumaki, \& Rosenthal (1972), and Soskin \& Kauffman (1961).

In most cases in which playing backwards, random splicing, and lowpass filtering have been used in recognition research as content masking techniques, they served to mask the content of speech obtained in a situation in which there were no constraints on what the speakers said. Another method which has been used to ensure that the verbal channel could vary freely without influencing the judgments of the listeners is to offer expressions of emotion in a foreign language, or, more precisely, in a language that the listeners cannot understand (cf. Brown, 1980; Kramer, 1964). This method can only be used with fluent bilinguals (one of the two languages preferably being a rather exotic one) who are able - and it is questionable whether this is altogether possible - to use the words and syntax of the foreign language while using the pitch, tempo, voice quality, etc. as they characterize emotions in the language of the listeners. It is essential that this be done since it is well known (see Chapter 9) that in different languages emotions may be expressed in different ways, not only verbally but also vocally.

In addition to the problem of how to separate the vocal and verbal channels a problem which is more important for recognition research than for descriptive research - there are a good many other reasons why so little use has been made of spontaneously produced emotional utterances. One reason is that such speech samples are very difficult to obtain. As Scherer states, "emotions are considered in most cultures private experiences which, at least in their extreme manifestations, are to be hidden or suppressed in public. Even if their expression is allowed, such as grief at a burial, it is usually in highly 
ritualized and ceremonial situations in which recording or filming or systematic observation are deemed inappropriate" (1981b, p. 201). (That it nevertheless is possible to find opportunities to record spontaneous instances of emotional speech has been demonstrated by Brown in a number of studies described above.)

The need for high quality recordings free of background noise, especially in those cases in which detailed acoustic analyses are to be carried out, has also discouraged the use of real-life utterances.

Another problem in using spontaneous speech is that it is hard to know what emotion the speaker was experiencing when producing it. This problem is particularly acute in cross-cultural research, since it is well known that the events which elicit a particular emotion may vary from culture to culture, and that there are different display rules, i.e., different norms regarding the expected management of emotional manifestations (for some examples and an extensive treatment of the subject, see Ekman, 1973). For recognition research this means that there is no objective criterion which can be used to determine the accuracy of listeners' responses. This problem has sometimes been avoided by giving the listeners the contexts in which the stimuli were produced as response categories. Moreover, researchers have sometimes contented themselves with establishing whether there is any system in the listeners" choice of a particular label when asked to describe the stimuli. But then, any system found might be caused by the fact that some labels are more likely to be chosen than others, for instance because they happen to be more frequently used in everyday language. Not knowing what emotion has been expressed is, of course, problematic in descriptive research as well.

A related problem, which has often been pointed out, is the fact that in everyday life "pure" emotions hardly occur; in most cases several emotions will be simultaneously present. Also, natural emotional behaviour tends to be rather mild as a result of display rules which deintensify, mask, or neutralize emotional expressions. To use Scherer's formulation again, "If the speech samples used in simulation studies are not natural enough, the samples consisting of spontaneous speech are not emotional enough" (1979, p. 512).

A final problem worth mentioning is the replication problem, the fact that many spontaneous utterances obtained may prove to be single cases, as it will be difficult to obtain other emotional expressions from the same speaker or expressions of the same emotion from other speakers.

In view of all the problems described above, it is not surprising that the vast majority of researchers have resorted to simulated expressions of emotion. It is then possible to use high quality equipment for the recordings and to exclude possible interactions between the verbal and vocal channels by having the speakers express the different emotions with speech samples with a fixed verbal content. As has been pointed out above, the use of simulated expressions of emotion is not only advantageous in recognition research - 
correct identification of the emotions expressed will then exclusively be based upon the correct interpretation of the vocal cues - but also in descriptive research since it enhances the comparability of the descriptions - any variation in the speech signal can be attributed to differences in emotional meaning. Moreover, by using the simulation method the problem of how to decide what emotion is expressed may be solved by taking the intention of the speaker as a criterion, the chance of obtaining "mixed" stimuli is made somewhat smaller, and the replication problem is taken care of.

Furthermore, it is quite reassuring that, at least for facial expressions of emotion, significant correlations have been found both between the abilities to decode posed and spontaneous stimuli, and the abilities to encode posed and spontaneous stimuli (Zuckerman, Hall, DeFrank, \& Rosenthal, 1976). Zuckerman et al. obtained the spontaneous expressions by videotaping subjects' faces while watching a number of unpleasant and pleasant videotaped scenes, and while talking about their reactions to the scenes. The facial segments were judged on a categorization measure, which required subjects to choose from among the videotaped scenes the one which they thought the sender was watching or talking about, and a pleasantness measure, which required subjects to judge how pleasant they thought the sender's feelings were. Accuracy of the judgments was defined both in terms of the original categorization of the videotaped scenes and the reaction to these as reported by the senders themselves.

As for vocal expressions of emotion, Williams and Stevens (1972) compared the original speech of the radio announcer describing the Hindenburg disaster (see above) with the speech of an actor who was asked to read a written version of the announcer's commentary as if he were in the announcer's position. Acoustic analyses revealed that in comparison to speech samples before the crash, speech samples after the crash, both in the original and simulated announcements, manifested irregular $F_{0}$ jumps, an increased $F_{0}$ median, and a wider $F_{0}$ range. In all three cases these acoustic phenomena were more clearly visible in the simulated than in the spontaneous speech. Thus, it looks as if simulated expressions have characteristics in common with spontaneous expressions. Moreover, if indeed emotional cues are more clearly present in simulated expressions - and this is not altogether unlikely in view of the purity and extremeness of simulated expressions - this could be taken as an argument to start by studying simulated expressions, and afterwards complement the findings with data derived from real-life behaviour.

Tables 3.7, 6.1, and 7.1 list, in addition to an occasional study with spontaneous speech samples or synthesized stimuli, the most relevant studies in which simulated vocal expressions of emotion have been used, and their methodology. It may be seen from these tables that a frequently used method in simulation studies is to ask a number of speakers, either professional actors, amateur actors, speech students, or persons without any dramatic or voice training, to produce a set of different emotions by counting, for 
example, from one to nine, or by reciting part of the alphabet. Other researchers have utilized meaningful, but presumably emotionally neutral speech samples of differing lengths, either single words, phrases, sentences, or paragraphs.

By keeping the speech material used as the carrier for the emotional expressions constant and assuming that its semantic meaning is emotionally unbiased, any emotional meaning inferred from the stimuli is deemed to have been transmitted via the vocal channel rather than via the verbal channel. Some standard passages have been particularly popular, such as the following, which, with slight modifications, has been used in a great number of studies: "There is no other answer. You've asked me that before. My reply has always been the same, and it will always be the same".

Often speakers have been asked to produce standard passages like these in isolation; in some instances standard passages have been inserted into different scripts each designed to fit the expression of a particular emotion, in an attempt to make the speakers' task a somewhat more natural one. Afterwards, the passages would then be taken out of their contexts again, either to be offered in a recognition experiment or to be described. Another attempt to obtain less artificial speech samples has been the mood induction technique in which the speakers are requested, for example, to first read an emotional poem or listen to selected music designed to put them in a specific emotional state before they produce the emotional utterances.

Not much is known about the relative efficacy of the different methods used in simulation studies and the consequences of the composition and the nature of the speech material for description and recognition. Problems inherent in mood induction are, in any case, that the range of different emotions which can be induced is rather limited, and that the effects of the technique may not be strong and/or long-lasting enough to show up afterwards in the voice.

With respect to the choice of the speech material, it is clear that the use of letters and numerals is very artificial. As for the different lengths of meaningful speech samples, samples consisting of one word seem to be too short to be able to contain all the different cues that might be relevant to emotional communication. On the other hand, a disadvantage of long samples, such as paragraphs, is that it may be hard for the speakers to sustain the emotional expressions throughout the speech sample. Therefore, we think that the best method would be to use a number of phrases or sentences.

As for the speakers, it seems safest to take people with at least some background in or inclination for acting since otherwise there is a fair chance that they will either be too embarrassed to fulfill their task or unable to differentiate among the expressions of various emotions.

On the basis of the arguments given above, in the present investigation we have chosen to examine the characteristics and recognizability of a set of 
simulated rather than spontaneously produced emotional expressions. The most common simulation method has been used, i.e., one in which the speakers were requested to express a number of emotions with a standard speech sample, in our case phrases. More details about the method used are given below.

\subsection{METHOD}

\subsubsection{Speakers}

A series of pilot experiments were conducted in which subjects were asked to express one or two phrases with nine different emotions, i.e., disgust, surprise, shame, interest, joy, fear, contempt, sadness, and anger, in addition to a neutral category. Five researchers from the Institute of Phonetics of the University of Nijmegen judged the expressions thus obtained on recognizability by indicating their judgment on a 5-point scale, in which $1=$ insufficient, $2=$ almost sufficient, $3=$ sufficient, $4=$ largely sufficient, and $5=$ good. Only those speakers were considered to be acceptable who, averaged over all expressions and all judges, score a 3 or higher. Twenty-three subjects were needed to obtain four males and four females who met this criterion.

The speakers selected were aged between 20 and 26 years. All were native speakers of Dutch and had attended an institute for higher education for at least one year. Most had had some experience with drama but not professionally. None of them had voice problems. The speakers were paid for their participation in the experiment.

\subsubsection{Phrases}

In the pilot experiments it appeared to be very difficult to find standard phrases which are both completely neutral with respect to their verbal content and suitable for expression with a wide range of different emotions. We considered it essential that every effort be made to ensure that the speakers would be able to differentiate between the emotions expressed, even if this meant selecting one or more phrases of which the verbal content might be associated more easily with some emotions than with others. This possibility was checked later in one of the recognition experiments (see Chapter 6).

In order to select the most suitable phrases the speakers participating in the pilot experiments were offered a set of eight phrases with the request to try and see which ones they had least problems with. There was general agreement that the following four phrases were most suitable:

1. Daar is ie weer (/dar is i ver/) ("there he is again")

2. Twee maanden zwanger (/tue manda zuanor/) ("two months pregnant")

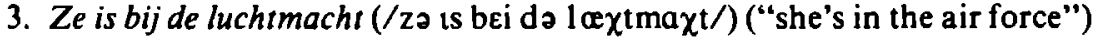

4. Zo'n grote Amerikaanse auto (/zon $\chi$ rota amerikansa oto/) ("such a big American car") 


\subsubsection{Instructions}

As it is commonly known that emotion labels, such as surprise, leave room for somewhat varying interpretations (pleasant surprise, unpleasant surprise, startled surprise, etc.), an attempt was made to narrow their meaning down by offering the speakers specific contexts in which to express the various phrases with the different emotions. This attempt failed because the suitability of the contexts appeared to be person dependent, and also because some speakers preferred not to use any context at all; they concentrated on the experience of a particular emotion and then "threw out" the phrase, or concentrated on the formal appearance of the emotional expressions. We therefore decided not to impose any constraints and to have the individual speakers themselves determine which method of expression suited them best. The only thing that was asked of them was to try and express each phrase with each emotion as unambiguously as they could, and to keep on trying until they were satisfied with the result or felt that further attempts would be futile. They were told, however, not to insert any extra words or noises, such as laughs or sobs, and to remain intelligible.

\subsubsection{Recordings}

In four different sessions with an interval of between one and seven days, the eight speakers which had been selected were asked to express the four phrases with the nine emotions mentioned above and also in a neutral way (in order to simplify further reading from now on neutral will also be referred to as an emotion). The recordings were made in an audio studio using a Sennheiser condensor microphone type 415 , a Studer audio console, and Studer tape recorders type $A$ 80/R. The distance between the mouths of the speakers and the microphone was held as constant as possible by placing a support pillow behind the heads of the speakers. It was sometimes necessary to attenuate the speech signal, particularly with loud expressions of anger and joy, in order to prevent overloading.

Immediately after each session the speakers listened to their own recordings to choose the best expression of each emotion with each phrase. The 4 (sessions) $\times 4$ (phrases) $\times 10$ (emotions) $=160$ best utterances of each speaker were spliced to one master tape which at the end of the fourth session was again presented to the speaker with the request to indicate the best expression of each emotion with each phrase. Thus, the final data base consisted of 8 (speakers) $\times 4$ (phrases) $\times 10$ (emotions) $=320$ emotional expressions.

These 320 expressions have all been used as stimuli in the recognition experiment with Dutch adults described in Chapter 6 . The other recognition experiments (Chapters 7,8, and 9), however, and the perceptual and acoustic descriptions (Chapters 3, 4, and 5) are based on different subsets of the speech material. Detailed information will be given in the respective chap- 
ters. However, in order to avoid redundancy, the reader will be referred back each time to the present chapter for information on the way in which the speech material has been obtained. 



\section{Perceptual characteristics of discrete emotions}

\subsection{INTRODUCTION}

From a survey of the literature it appears that not much is known yet about the characteristics of vocal expressions of discrete emotions, only a few emotional categories having been more or less systematically investigated, and then only with respect to a limited number of vocal parameters. Moreover, it appears that most descriptions pertain to emotional utterances produced by American English speakers; the data on other languages are very scanty and scattered (see Table 3.7).

The major objective of the study presented in this chapter was to obtain an extensive perceptual description of a large number of vocal expressions of discrete emotions in order to establish their characteristic features and to compare these with findings from other studies. We were also interested in whether the realizations of the various emotions varied as a function of the sex of the speaker and the phrase with which they were uttered. A methodological point of interest was the interrater reliability of the ratings.

One of the reasons why the study of the vocal characteristics of emotional speech has been so limited - and this holds for perceptual as well as acoustic descriptions, the choice of specific acoustic parameters often being determined by the search for correlates of specific perceptual parameters - has been the lack of a comprehensive system for the description of vocal phenomena. The available coding schemes (e.g. Crystal, 1969, 1975; Crystal \& Quirk, 1964; Trager, 1958) have been rarely used in empirical research into emotional characteristics, for a great part as a result of the fact that the categories included in them are often not defined explicitly enough to permit an unambiguous interpretation. Another reason may be that the inventory of phenomena covered often makes a somewhat ad hoc impression because of the lack of a theoretical framework within which the schemes are placed (for some critical remarks, see also Scherer, 1982, p. 146). Moreover, few data have been reported on the intra- and interpersonal reliability of the application of the categories included in the schemes developed.

However scarce the data on the characteristics of vocal expressions of discrete emotions may be, it looks as if at least some of the emotions for 
which universal facial configurations have been found are realized vocally fairly consistently also, both within and across cultures, at least with respect to the few vocal features which have been more or less systematically examined. Thus, it has been found that sadness is generally characterized by low pitch, soft voice, and slow tempo, and anger and joy by high pitch, loud voice, and fast tempo. Many more descriptive data are badly needed though, both to extend the range of features on which attention has hitherto been focused (i.e., pitch level, pitch range, tempo, and, to a lesser degree, loudness) and in order to assess whether consistent vocal patterns may be found for other than the few emotions which have been considered. More extensive descriptive research is essential to determine the extent to which the vocal characteristics of emotions are culture-specific and/or arbitrary and the extent to which they are universal and/or physiologically determined. Knowledge in this area may eventually lead to insight into the innate component of emotional manifestations.

Most of the perceptual parameters used in the present study for describing Dutch realizations of ten different emotions have been derived from one of the earlier drafts of the perceptual protocol for the auditory description of abnormal voices found in speech disorders which has been developed by Laver and his co-workers (Laver, Wirz, MacKenzie, \& Hiller, 1981). This protocol is an extension of what has been called "the first theoretically consistent and well-documented system of categories for the assessment of voice quality on the phonatory-articulatory level" (Scherer, 1982, p. 148), which Laver had originally developed for the normal voice (Laver, 1968, 1975, 1979, 1980; Laver \& Hanson, 1981; Laver \& Trudgill, 1979).

The basic unit of the scheme developed by Laver is the auditory impression rendered by a voice "setting", which is a habitual tendency towards maintaining particular muscular adjustments, of the vocal tract and/or the larynx. Examples of voice settings are the tendency to speak with lip spreading (a supralaryngeal setting) or with a creaky mode of phonation (a laryngeal setting). Each such component/setting is defined in terms of its deviation from an acoustically and articulatorily specified neutral configuration of the vocal apparatus. The perceptual product of constellations of such components/settings in the speech of a given speaker makes up his vocal profile.

The notion of setting has been developed in phonetic theory mainly to describe relatively permanent vocal effects acting as individuating markers, i.e., distinguishing the habitual voice of a particular speaker from that of other speakers, as social markers, i.e., distinguishing the habitual voice of a group of speakers from that of other groups of speakers (van Bezooijen, 1983; Esling, 1978; Trudgill, 1974), or as linguistic group membership markers, i.e., distinguishing the habitual articulatory tendencies of speakers of different languages (Honikman, 1964; Scherer, 1974). Many of the same vocal phenomena, however, which provide extralinguistic information, i.e., information on the (quasi-)permanent characteristics of speech, may also be 
employed for paralinguistic purposes, e.g. for the communication of affect (Laver \& Trudgill, 1979, p. 15-16). Therefore, it is possible to use a number of the categories proposed by Laver for the phonatory-articulatory description of relatively permanent vocal effects in a study, such as ours, of the more temporary vocal characteristics of emotional expressions.

The scheme developed by Laver for the description of the normal voice placed a strong emphasis on voice quality settings. In the more recent and revised schemes aimed at describing abnormal voices, in addition to voice quality features, a few prosodic features, related to pitch, loudness, and rate, have been included (see the analysis protocol given in Laver et al., $1981 \mathrm{p}$. 140-141). Some of the latter features have also been rated in the present study.

\subsection{METHOD}

The perceptual description was obtained by having a small panel of raters score vocal expressions of emotion in Dutch on a selected set of vocal parameter scales.

\subsubsection{Speech material}

The speech material consisted of a subset of the recorded material, namely 160 emotional expressions, consisting of 8 speakers ( 4 males and 4 females) $\times$ 2 phrases (twee maanden zwanger and zo'n grote Amerikaanse auto) $\times 10$ emotions (neutral, disgust, surprise, shame, interest, joy, fear, contempt, sadness, and anger). (For a more detailed description of the speech material, see Chapter 2.) Only half of the material was described because a description of the full set of $\mathbf{3 2 0}$ utterances would have been too time consuming.

\subsubsection{Raters}

The emotional expressions were rated by six persons, five females and one male, all between 23 and 28 years of age. One was the present author, two were graduate students of phonetics, two were graduate students of Dutch, and one was a graduate student of applied linguistics. The two students of phonetics were paid for their participation; the three other students participated in partial fulfillment of their degree requirements. None of the raters knew any of the speakers.

\subsubsection{Parameters}

The 160 emotional expressions were rated on thirteen scales, namely:

1. Lip rounding (roun) $(0=$ none, $5=$ extremely rounded)

2. Lip spreading (spre) $(0=$ none, $5=$ extremely spread) 
3. Laryngeal tension (tens): squeezed and tight quality $(0=$ none, $5=$ extremely tense)

4. Laryngeal laxness (laxn): sonorous and resonant quality $(0=$ none, $5=$ extremely lax)

5. Creak (crea): glottal or vocal fry ( $0=$ none, $5=$ extremely creaky)

6. Tremulousness (trem): trembling quality $(0=$ none, $5=$ extremely tremulous)

7. Whisper (whis) $(0=$ none, $5=$ extremely whispery)

8. Harshness (hars): rough and rasping quality $(0=$ none, $5=$ extremely harsh)

9. Pitch level (pitc): global impression of the average pitch level, related to the sex of the speaker $(1=$ extremely low, $7=$ extremely high $)$

10. Pitch range (rang): distance between the lowest and the highest pitch (1 $=$ extremely narrow, $7=$ extremely wide)

11. Loudness (loud): impression of the original absolute loudness (all recordings were brought to the same intensity level (see Section 2.2.4)) $(1=$ extremely soft, $7=$ extremely loud)

12. Tempo (temp): articulation rate, i.e., the amount of speech produced per time unit, disregarding the pauses if any $(1=$ extremely slow, $7=$ extremely fast)

13. Precision of articulation (prec): precise articulation would be characterized by the presence of less assimilation, tenser vowels, and longer and more fortis consonants than imprecise articulation ( 1 = extremely imprecise, $7=$ extremely precise)

Parameters 9 to 13 were rated on bipolar 7-point scales, going from one extreme via a neutral midpoint to the opposite extreme; these parameters are an intrinsic part of the speech signal. Parameters 1 to 8 were rated on unipolar 6-point scales, going from the paralinguistic absence of the feature in question to a high degree of presence; these parameters can only depart from the neutral point of reference into one direction. Exceptions are laryngeal tension and laryngeal laxness. These two scales were taken in their original form from Laver (1981) (we admit it would have been more logical to combine these two scales to one; in later research this has in fact been done).

All but one of the 6-point scales $(1,2,3,4,5,7,8)$ have been taken from an earlier version of the protocol used by Laver et al. (1981) for the description of abnormal voices. However, we have not adopted the division between normal and abnormal separating scalar degrees 2-4 from scalar degrees 5-7, firstly because, as Laver et al. pointed out themselves, "the normal/abnormal division is somewhat problematic" $(1981$, p. 145) and secondly because the distinction is not relevant outside a context of treatment.

At the time that we selected our parameters, parameter 6 (tremulousness) did not figure in the protocol used by Laver et al. We included it in our inventory because of indications in the literature (Fairbanks \& Pronovost, 1939) that this feature might be characteristic of some emotions in the 
stimulus material. It was later added by Laver et al. to their scheme also, in the form of "tremor". The same holds true, more or less, for four of the other parameters that we have selected for use in the present study, namely parameters 9, 10,11, and 12: At the time that we drew up our inventory these parameters were not systematically used by Laver et al., but they were later added to their scheme. In emotion research these four parameters are the vocal characteristics which have been studied most frequently, in contrast to Laver's approach generally by means of ratings on bipolar scales. We decided to follow this tradition. For a detailed acoustic and articulatory description of parameters 1, 2, 5, 7, and 8, we refer to Laver (1980); a description of parameters 3 and 4 is given by Scherer (1974, p. 287). Parameter 13 was included because it had been found by Williams and Stevens (1972) to differentiate between at least some of the emotions examined. This parameter is discussed by Scherer $(1974$, p. 286$)$.

\subsubsection{Rating}

The rating sessions were preceded by training sessions which aimed at developing a common frame of reference and common anchor points for the different scale positions on the different parameter scales. This was done by listening to expressions of emotion not belonging to the corpus of utterances to be scored in the rating sessions but which were produced by the same speakers, and by comparing and discussing the ratings of the individual raters in the group. Use was also made of a demonstration tape (Laver, 1980). The scoring of each parameter was practiced for about 10 minutes, which means that the raters must be considered as slightly trained.

The 160 utterances used in the rating sessions were randomized per speaker and per phrase. In each session the utterances were rated on two or three parameters. The utterances were repeated until all raters had recorded their scores. During these sessions the raters were not allowed to discuss their ratings. They were asked to rate the vocal characteristics of the emotional utterances in relation to the anchor points established in the training sessions and not as deviations from the habitual voice of the speakers. When rating pitch level, the sex of the speaker was taken into account. Parameters 1 and 2 (lip rounding and lip spreading) were mutually exclusive, i.e., only one of the two could be rated for any one expression. The same was the case for parameters 3 and 4 (laryngeal tension and laryngeal laxness).

\subsection{RESULTS}

We will first present the reliability of the ratings. Then, after having assessed whether there was a systematic relationship between the scores on the different parameter scales and the sex of the speakers, the phrases, and - most importantly - the emotional categories expressed, and also after having 
established to what extent the ratıngs on the various parameters vanied independently of each other, the results will be given with respect to the charactenstic vocal patterns that we have found for the different emotions

\subsection{Reliability}

In order to assess the reliability of the means of the ratıngs given by the six raters on each of the thirteen parameter scales use was made of the intraclass correlation coefficient computed according to the method given in Winer (1971, p. 287, formula 7) The coefficients are given in Table 3.1 Since the reliability data show that the agreement among the raters is quite satısfactory, the individual ratıngs were combined to means for the subsequent analyses.

Table 3 I Relıability of the ratıngs on thirteen vocal parameter scales for 160 emotıonal expressions

Lip rounding

Lup spreading

Creak

Tremulousness

Whisper

Harshness

Pitch level

Pitch range

\subsubsection{Factors sex of speaker, phrase, and emotion}

The mean ratings on each of the thirteen parameters were subjected to $2 \times 2$ $\times 10 \times 8$ analyses of variance with three fixed factors, namely sex of speaker (A), phrase (B), and emotion (C), and one random factor, namely speakers. $A$ repeated measurements design was used with speakers nested under the factor sex of speaker. A was tested against subjects within groups, $B$ and $A X$ $B$ against $B \times$ subjects within groups, $C$ and $A \times C$ against $C \times$ subjects within groups and $B \times C$ and $A \times B \times C$ against $B \times C \times$ subjects withın groups. We adopted a level of significance of $5 \%$. The results are given in Table 3.2.

\subsubsection{Factor sex of speaker}

As may be seen from Table 3.2, for only two parameters, namely laxness and harshness, a significant effect for the factor sex of speaker was found. 
Table 32 Analyses of variance based on the mean ratıngs on thirteen vocal parameter scales for ten emotions expressed with four phrases by male and female speakers

\begin{tabular}{|c|c|c|c|c|c|c|c|c|c|c|c|c|c|c|c|}
\hline \multicolumn{2}{|c|}{ Source of variation } & \multirow[t]{2}{*}{$d f$} & \multicolumn{13}{|l|}{$F$-ratio } \\
\hline & & & Roun & Spre & Tens & Laxn & Crea & Trem & Whis & Hars & Pitc & Rang & Loud & Temp & Prec \\
\hline Sex of speaker & (A) & 1,6 & 320 & 025 & 291 & $1215^{*}$ & 000 & 084 & 000 & $109 l^{*}$ & 287 & 014 & 075 & 108 & 122 \\
\hline Phrase & (B) & 1,6 & $2608^{\circ}$ & $1431^{*}$ & 000 & 010 & $629 *$ & 586 & 077 & 413 & 224 & 454 & 001 & 064 & 029 \\
\hline Emotion & (C) & 9,54 & 202 & $269^{*}$ & $2000^{\circ}$ & $2451^{*}$ & $660^{*}$ & $450^{*}$ & $494^{*}$ & $341^{*}$ & $1597^{\circ}$ & $1140^{*}$ & $2793^{*}$ & $931 *$ & $354^{*}$ \\
\hline $\mathbf{A} \times \mathbf{B}$ & & 1,6 & 011 & 027 & $616^{*}$ & 047 & 136 & 046 & 153 & 218 & 004 & 081 & 125 & 007 & 023 \\
\hline $\mathbf{A} \times \mathbf{C}$ & & 9,54 & 078 & 135 & 179 & $302^{*}$ & 056 & 195 & 177 & 160 & 219 & 146 & 168 & 082 & 066 \\
\hline $\mathbf{B} \times \mathbf{C}$ & & 9,54 & 078 & 112 & 089 & 068 & $236^{*}$ & $278 *$ & 180 & 101 & 099 & 187 & 155 & 060 & 077 \\
\hline $\mathbf{A} \times \mathbf{B} \times \mathbf{C}$ & & 9,54 & 102 & 084 & 096 & 038 & 051 & $282^{*}$ & 150 & 186 & 103 & 056 & 105 & 077 & 111 \\
\hline
\end{tabular}

* $p<05$ 
Inspection of the cell means revealed that the emotional expressions of the male speakers were perceived to be more lax and more harsh than those of the female speakers. For either parameter the lowest score for any of the four male speakers, averaged over the two phrases and the ten emotions, was higher than the highest score, averaged over the two phrases and the ten emotions, for any of the four female speakers. For the other parameters the conclusion may be drawn that the perceptual scores have not been influenced in a systematic way by the sex of the speaker. There was only one significant interaction between sex of speaker and emotion, namely in the case of laryngeal laxness, and only one significant interaction between sex of speaker and phrase, namely in the case of laryngeal tension. As for the latter interaction, it appeared that the phrase $t$ wee maanden zwanger had been perceived to have been produced with an equal amount of tension by the male and the female speakers, whereas for zo'n grote Amerikaanse auto the male realizations received much higher tension scores than the female ones.

\subsubsection{Factor phrase}

The factor phrase had a significant effect in the case of three parameters, namely lip rounding, lip spreading, and creak. As for the first two parameters, it appeared that the phrase zo'n grote Amerikaanse auto had been perceived more often and to a higher degree to have been produced with lip rounding, while the realizations of twee maanden zwanger had been perceived to be more spread. As for creak, it appeared that the former had received higher ratings than the latter.

\subsubsection{Factor emotion}

With regard to the factor emotion - the factor we are most interested in - it appears that there is a significant effect on the scores for all parameters except lip rounding, which was therefore excluded from further analysis. In order to gain insight into the source of this effect - after all, theoretically it would, for example, be possible that in all cases it was one and the same emotional category that differed from all the others - Duncan's new multiple range test (Kirk, 1968, p. 93-94) was carried out, separately for each of the twelve parameters for which a significant effect of the factor emotion had been found. Duncan's test is an a posteriori contrast test; it is a systematic procedure for comparing all possible pairs of group means (in this case the mean scores for each emotion, averaged over speakers and phrases), and for determining the significance of the differences among them.

The outcomes of the test suggest that the twelve parameters may be roughly divided into two groups. In the one group, comprising lip spreading, laryngeal laxness, creak, tremulousness, whisper, harshness, and precision of articulation, a few emotional categories differ significanty from all or most of the other categories: joy (an exceptionally clear example) in the case of lip spreading; neutral, shame, and interest in the case of laryngeal laxness; sadness and shame in the case of creak; sadness and fear in the case of 
whisper; joy, and to a minor degree, disgust and anger in the case of harshness; and sadness in the case of precision of articulation. In the other group, comprising laryngeal tension, pitch level, pitch range, loudness, and tempo, it is not so much one or two emotional categories differing significantly from the rest, but rather most categories differing significantly from at least a number of other categories. For this group it is much harder to characterize the emotional categories.

In any case, these results suggest that most or all of the twelve parameters may contain information on particular emotions expressed. In order to gain additional information on the relationships among the different parameters, and especially in order to determine to what extent the parameters vary independently of each other, product-moment correlations were computed (Table 3.3). The level of significance was set at $5 \%$.

It appears from Table 3.3 that there are quite a few significant correlation coefficients. Most of them are not very high though, especially if one thinks in terms of the amount of variance in one variable accounted for by any of the other variables $\left(r^{2}\right)$. Only in three cases, which are more or less to be expected on physiological grounds, did we find fairly high correlations between pairs of variables, namely a negative one between laryngeal tension and laryngeal laxness (-.67), and positive ones between laryngeal tension and loudness (.71) and between laryngeal tension and harshness (.65).

The fact that the factor emotion was found to have a significant effect on the scores for twelve vocal parameters, the fact that an a posteriori contrast test revealed that this effect was not due to one or two emotions being different in all respects from the rest, and the fact that very few of the parameters correlate highly with each other suggest that each of these twelve parameters might be relevant to distinguishing the ten emotional categories examined. In order to get an impression of the typical characteristics of the different emotions the scores on the twelve parameters were averaged over speakers and phrases, separately for each emotional category. The mean scores on each of the twelve parameters and the standard deviations are presented in Table 3.4.

If we try to combine - for some parameters such as laryngeal tension, pitch level, pitch range, loudness, and tempo somewhat tentatively - the data given in Table 3.4 with the results of the a posteriori test mentioned above, it appears that only one emotion is characterized by the presence of lip spreading namely joy, which is, of course, what one would expect because of the smile which often accompanies expressions of joy. Anger is characterized by great laryngeal tension, whereas neutral, shame, and interest are characterized by great laryngeal laxness. Sadness is characterized by creak; fear and sadness by tremulousness; shame and fear by whisper; and disgust, joy, and anger by harshness. It may further be seen that joy is characterized by a high pitch, and neutral and disgust by a low pitch; that surprise and interest are characterized by a wide pitch range, and sadness and shame by a narrow 
Table 3.3 Intercorrelations between twelve vocal parameters $(N=160)$

\begin{tabular}{|c|c|c|c|c|c|c|c|c|c|c|c|}
\hline & Spre & Crea & Trem & Whis & Hars & Tens & Laxn & Pitc & Rang & Loud & Temp \\
\hline Crea & -.08 & & & & & & & & & & \\
\hline Trem & .07 & .06 & & & & & & & & & \\
\hline Whis & -.09 & .14 & .05 & & & & & & & & \\
\hline Hars & .00 & -.02 & -.05 & 06 & & & & & & & \\
\hline Tens & $.17^{\star}$ & -.15 & .09 & -.02 & $.65^{*}$ & & & & & & \\
\hline Laxn & -.14 & -.03 & $-19 *$ & -.06 & $-.29^{*}$ & $-.67 *$ & & & & & \\
\hline Pitc & .24 & $-.36^{*}$ & .15 & -.07 & -.01 & $.41 *$ & $-.40^{\circ}$ & & & & \\
\hline Rang & .03 & $-.24^{*}$ & $-.20^{*}$ & -.07 & .12 & .11 & $-.16^{*}$ & $.24^{*}$ & & & \\
\hline Loud & $.18^{*}$ & $-.36^{\star}$ & -.07 & $-.34^{\star}$ & $.46^{*}$ & $.71^{*}$ & $-.45^{\star}$ & $.37^{*}$ & $.36^{*}$ & & \\
\hline Temp & .04 & $-.33^{*}$ & .00 & -.05 & $-.17^{*}$ & .01 & -.03 & $.43^{*}$ & .06 & .14 & \\
\hline Prec & .09 & -.09 & -.18 & -.12 & -.10 & .10 & .08 & -.15 & $.23^{*}$ & .05 & $-.23^{*}$ \\
\hline
\end{tabular}

$*<.05$ (two-talled test) 
Table 34 Mean scores and standard deviations (in parentheses) for ten emotions on twelve vocal parameter scales

\begin{tabular}{|c|c|c|c|c|c|c|}
\hline & Spre (0-5) & Tens $(0-5)$ & $\operatorname{Laxn}(0-5)$ & Crea (0-5) & Trem (0-5) & Whis $(0-5)$ \\
\hline Neutral & $007(033)$ & $003(023)$ & $230(156)$ & $052(078)$ & $001(010)$ & $064(0$ 10) \\
\hline Disgust & $041(078)$ & 222 (1 42) & $004(029)$ & $109(142)$ & $005(027)$ & $106(132)$ \\
\hline Surprise & $030(070)$ & 2.29 (1 31) & $026(071)$ & $031(080)$ & $004(020)$ & $104(121)$ \\
\hline Shame & $005(027)$ & $020(047)$ & $150(148)$ & $116(133)$ & $006(024)$ & $146(139)$ \\
\hline Interest & $009(054)$ & $056(079)$ & $077\left(\begin{array}{ll}1 & 13\end{array}\right)$ & $020(045)$ & $002(014)$ & $077(092)$ \\
\hline Joy & $097(148)$ & $262(147)$ & $012(057)$ & $019(065)$ & $026(074)$ & $049(086)$ \\
\hline Fear & $025(085)$ & $260(127)$ & $000(000)$ & $033(080)$ & $059\left(\begin{array}{ll}1 & 01)\end{array}\right)$ & $178(150)$ \\
\hline Contempt & $034(075)$ & $164(129)$ & $040(101)$ & $076(125)$ & $022(058)$ & $057(083)$ \\
\hline Sadness & 027 (0 57) & $150(120)$ & $018(063)$ & $210(159)$ & 057 (0 94) & $078(099)$ \\
\hline \multirow[t]{2}{*}{ Anger } & $024(070)$ & $335(100)$ & $000(000)$ & $020(064)$ & $009(044)$ & $040(069)$ \\
\hline & Hars $(0-5)$ & Pitc (1-7) & Rang (1-7) & Loud (1-7) & Temp (1-7) & Prec (1-7) \\
\hline Neutral & $004(020)$ & $346(056)$ & $371(061)$ & $398(029)$ & $409(039)$ & $442(077)$ \\
\hline Disgust & $107(148)$ & $347(094)$ & $400(088)$ & $442(078)$ & $296(092)$ & 422 (1 15) \\
\hline Surprise & $078(130)$ & $509(109)$ & $558(098)$ & $485(074)$ & $412(084)$ & $403(076)$ \\
\hline Shame & $004(029)$ & $359(076)$ & $338(0$ 85) & $290(067)$ & $368(085)$ & $398(075)$ \\
\hline Interest & $002(0$ 14) & $488(058)$ & $477(076)$ & $417(054)$ & $445(072)$ & $423(045)$ \\
\hline Joy & $116(147)$ & $556(079)$ & $436(087)$ & $527(070)$ & $432(089)$ & $393(071)$ \\
\hline Fear & $050\left(\begin{array}{ll}1 & 22\end{array}\right)$ & $524(066)$ & $388(088)$ & $407(099)$ & $454(095)$ & $381(070)$ \\
\hline Contempt & $032(067)$ & $368(134)$ & $433(130)$ & $458(061)$ & $343(099)$ & $435(082)$ \\
\hline Sadness & $027(066)$ & $446(084)$ & $306(100)$ & $377(088)$ & $374(071)$ & $356(086)$ \\
\hline Anger & $100(134)$ & $448(088)$ & $430(087)$ & $555(063)$ & $427(083)$ & $438(074)$ \\
\hline
\end{tabular}


pitch range; that joy and anger are characterized by a loud voice, whereas a soft voice is characteristic of shame; that a slow tempo is characteristic of disgust and contempt; and, finally, that sadness is characterized by imprecise articulation. These observations are presented in Table 3.5, grouped together per emotional category.

Table 35 Characteristic features of Dutch expressions of ten emotions

\begin{tabular}{ll}
\hline Neutral & Great laryngeal laxness, low pitch level \\
Disgust & Harshness, low pitch level, slow tempo \\
Surprise & Wide pitch range \\
Shame & Great laryngeal laxness, narrow pitch range, soft voice \\
Interest & Great laryngeal laxness, wide pitch range \\
Joy & Lip spreading, harshness, high pitch level, loud voice \\
Fear & Tremulousness, whisper \\
Contempt & Slow tempo \\
Sadness & Creak, tremulousness, narrow pitch range, imprecise articulation \\
Anger & Great laryngeal tension, harshness, loud voice \\
\hline
\end{tabular}

\subsection{DISCUSSION}

\subsection{Reliability}

In the present study high interrater reliability coefficients were obtained for the ratings by six slightly trained raters (on the average only 10 minutes of training per parameter) on the parameters of lip rounding, lip spreading, laryngeal tension, laryngeal laxness, creak, tremulousness, whisper, harshness, pitch level, pitch range, loudness, tempo, and precision of articulation. That pitch level, loudness, and tempo could be reliably assessed perceptually has been known for quite some tıme (Constanzo, Markel, \& Constanzo, 1969, Davitz, 1964c, Duncan \& Rosenthal, 1968; Huttar, 1968; Markel, 1965). More recently, Scherer (1978), using phonetıcians as raters, found a rather high reliability for pitch level, pitch range, loudness, precision of articulation, creak, and a number of other parameters not included in the present study, such as dynamic range and breathıness. He found a very low coefficient, however, for laryngeal tension. In the same study Scherer obtalned a high reliability with untrained undergraduate students for harshness, loudness, and pitch. Hammarberg et al (1980), using one phoniatrician and thirteen speech therapists (who had worked together for some ten years) as raters, found high reliabilities for, among other things, creak, harshness, whisper, and tremulousness. Tartter (1980) reports that non-trained high school and college students identıfied lip spreading significantly more often than chance.

Laver et al. (1981), instead of computıng reliability coefficients, assessed 
the "accuracy" of the ratings of two groups of ten speech therapists each on a wide range of settings. One group had been trained on a two-and-a-half day program and the other on an eight week, one-and-a-half hour session per week program. The stimulus material consisted of six rather deviating voices. Accuracy was defined in terms of the mean deviations of the ratings from the agreed upon judgments of a panel of three raters who had an experience of many years in the use of the system, had been closely involved in its development, and, in many cases, were informed about the physiological origin of the speech defects they described. For both groups the rating of whisper, creak, harshness, lip rounding/spreading, laryngeal tension, and many other settings not employed in the present study was found to be acceptable, i.e., on the average less than two scalar degrees removed from the panel score. It should be noted, however, that not all settings were present in the stimulus material, so that the results might be somewhat flattering (it is probably easier to agree on the absence of a feature than on the degree in which a feature is present).

In general, these data suggest that a fairly reliable perceptual description comprehending quite an extensive number of vocal characteristics may be obtained if well trained raters are used, and also if slightly trained or (large groups of) untrained raters are used. However, it should be kept in mind that the reliability of ratings may be affected by various factors. In our stimulus material, for example, covering the expressions of a wide variety of different emotions, for most parameters the range spanned by the utterances was quite wide, and this is known to raise reliability. This will also be the case with material in which both healthy and pathological voices are included. Moreover, in our material the task of the raters was facilitated by the fact that the verbal content of the speech samples was held constant, the fact that there were a number of utterances produced by one and the same speaker which were each time presented consecutively, and the fact that in each session only a few parameters were scored (in most other studies each speech sample is rated on all parameters simultaneously). The length of the utterances to be described probably has a differential effect on the rating of the different parameters. We had the impression that in our material the rating of pitch level, pitch range, loudness, and tempo may have been relatively easy because the utterances were too short to manifest much variation, whereas creak, for example, was relatively difficult to rate because the utterances were not long enough to give a good impression of the relative occurrence and strength of this parameter. It should also be noted that there are many different measures for assessing reliability, sometimes yielding quite divergent results (for a comparison of different measures, see Asendorpf \& Wallbott, 1979).

\subsection{Factor sex of speaker}

Analyses of variance carried out on the mean ratings for each of the 160 
utterances, separately for each of the thirteen parameters, hardly yielded any significant effects for the factor sex of speaker. Other researchers who have reported descriptions of vocal expressions of emotion have either not been interested in differences between the realizations of their male and female speakers, or have not tested the significance of the differences found (Skinner, 1935; Kaiser 1962). Davitz, (1964c) seems to be the only exception. He had the expressions of fourteen different emotions, expressed by four female and three male speakers, rated on four speech scales, namely loudness, pitch level, timbre, and rate. He found that these vocal characteristics varied over the fourteen emotional expressions in about the same way for males and females.

The few differences that we did find, namely greater laxness and more harshness for the male speakers, were so consistent for the individual speakers that they may be characteristic of the difference between female and male speech in general. At least for harshness, this is confirmed by the impression that harsh voice is heard much less commonly in women than in men (Laver, 1980, p. 128).

\subsubsection{Factor phrase}

The fact that a significant effect of the factor phrase on the ratings for lip rounding and lip spreading was found, may be explained by the fact that, in our experience, extra (para- or extralinguistic) lip rounding is easier to detect in rounded vowels, whereas extra lip spreading is easier to detect in unrounded vowels. As a consequence, $/$ zon $\chi$ rot $ə$ amerikans $ə$ oto/, containing four rounded vowels three of which can be stressed, would be likely to attract higher scores on the scale for lip rounding than /tve mandə zuajər/, a phrase which does not contain a single rounded vowel. Similarly, /tue mando zuanar/, containing three unrounded vowels which can be stressed would be more likely to attract higher scores on the scale for lip spreading. This is exactly what we found. It must be said, however, that in this particular case our experience does not agree with Laver's views as to the kind of key segments that would be most susceptible to the effects of lip settings; according to him, rounding would be most audible on spread vowels, especially the closed ones such as $/ \mathrm{i} /$, whereas spreading would be most easily perceived with rounded vowels such as the / $u$ / (Laver, 1981).

In any case, the conclusion seems warranted that it is not sufficient to ensure that the verbal content of the standard material used in simulation studies is emotionally unbiased or fit to be produced with a wide range of different emotions. Apparently it is also advisable to pay due attention to the phonetic properties of the speech material in order to prevent undesirable interactions between speech material and the parameters used in the investigation. One would have to take care, for example, to include an equal number of rounded and spread vowels in different phrases in order for them to be completely comparable. On the other hand, the emphasis on perfectly 
phonetically balanced speech material should not lead to the construction of texts that are so unnatural and lengthy as to make any emotional rendering of them impossible.

\subsubsection{Perceptual characteristics of emotions}

As for the characteristics of the various emotions examined in the present study (see Table 3.5), neutral was found to be characterized by a high degree of laryngeal laxness and a low pitch level; disgust by harshness, a low pitch level, and a slow tempo; surprise by a wide pitch range; shame by great laryngeal laxness, whisper, a narrow pitch range, and a soft voice; interest by great laryngeal laxness and a wide pitch range; joy by lip spreading, harshness, a high pitch level, and a loud voice; fear by tremulousness and whisper; contempt by a slow tempo; sadness by creak, tremulousness, a narrow pitch range, and imprecise articulation; and anger by great laryngeal tension, harshness, and a loud voice. Most of these characteristics emerged clearly from an a posteriori contrast test in which for each parameter a pairwise comparison was made among the mean scores for each emotion. Nevertheless, it should also be noted that for the characteristics mentioned the variance of the scores around the means was generally rather high, which means that these characteristics have not been produced very consistently.

It would of course, be interesting to see to what extent our findings with regard to the characteristics of vocal expressions of emotion in Dutch correspond with the outcomes of previous research pertaining to other languages. Unfortunately, it is impossible to make more than a partial and tentative comparison.

The comparison can only be partial because not all emotions and very few parameters have been studied in previous research. It can only be tentative because of the fact that varying speech material has been used, the fact that it has sometimes been produced by only one or two speakers, and most of all because the stimuli have been described in different ways. The last problem concerns both the interpretation of acoustic and perceptual data. Little research has been done into the relationship between acoustic and perceptual variables, particularly in running, non-neutral speech (this problem is dealt with in Chapter 5). Hence it is often not clear in what way acoustic findings should be interpreted and translated into perceptual terms. Patterns of vocal characteristics of emotional expressions stated in perceptual terms are often hard to interpret because of the lack of consistency in the terminology used breathy and whispery, for example, sometimes refer to the same phonatory and acoustic phenomenon, and sometimes to separate phenomena - and because of the absence of reliability data.

For the comparison between our findings and the outcomes of other studies we have only made use of those acoustic and perceptual measures of which it is commonly assumed that they are more or less directly related to or comparable with a specific parameter employed in our study. Some diffe- 
rences between measures, such as the fact that tempo has sometimes been defined as speaking rate (including pause time) and sometimes as articulation rate (excluding pause time), and the fact that the acoustic correlate of pitch level has sometimes been defined as $F_{0}$ mean and sometimes as $F_{0}$ median, have been disregarded. Had we been more strict, there would have been very little to compare.

With regard to most of our parameters, no or very few data are available for comparison. The only parameters on which more or less systematic data are available, either in perceptual or acoustic terms, are pitch level (average fundamental frequency), pitch range (fundamental frequency range), tempo (normalized duration), and, to a lesser degree, loudness (intensity or energy distribution in the voice spectrum). Since these data have often been presented verbally, for example in terms of high, mid, and low pitch, rather than numerically, either in $\mathrm{Hz}$, semitones, $\mathrm{dB}$, or sec, or in the form of scale positions, we were forced to divide the scales that we employed for the rating of these four parameters into similar categories in order to allow comparison. Unfortunately, these very parameters happened to be among the ones for which the a posteriori comparison test described above had yielded results which were hard to interpret in terms of categorical differences between (groups of) emotions. In the absence of formal criteria, we decided to proceed as follows: Emotions that on the average had received a score above scale position 4.2 were considered to be high (with regard to pitch level), wide (with regard to pitch range), loud, or fast, and emotions that on the average had received a score below scale position 3.8 were considered to be low, narrow, soft, or slow, respectively. Emotions for which the average score fell in between were interpreted as mid. This is, of course, a rather arbitrary procedure, but then, any other procedure would have been equally arbitrary.

Even so it is hard to compare results. The way in which researchers have assigned different emotions to different categories is far from consistent and is often not explicitly mentioned. In some cases emotional expressions have been related to each other (as in our case), in other cases to some non-defined baseline or to neutral speech. A drawback in taking neutral speech as a reference is that, as we have seen above, it does not occupy a "neutral" position on all parameter scales: In the present study, for example, neutral was characterized by the lowest pitch level and the most precise articulation of all categories examined. This finding is very intriguing - it suggests that speakers and raters may have had divergent ideas on what the term neutral stands for - but also rather annoying. It could mean, for example, that what is considered high pitch in one study may well be considered low pitch in another, depending on the point of reference used, and, if the emotions are contrasted with one another, on the specific combination of emotions that happens to be examined. In those cases in which raw data are provided, it is not always clear what categorization criteria should be used either. 
Table 3.6 Comparison of the results on pitch level, pitch range, loudness, and tempo for ten emotions. The numbers in parentheses refer to the studies included in Table 3.7.

\begin{tabular}{|c|c|c|c|c|c|c|c|c|}
\hline & \multicolumn{2}{|c|}{ Pitch level } & \multicolumn{2}{|c|}{ Pitch range } & \multicolumn{2}{|c|}{ Loudness } & \multicolumn{2}{|l|}{ Tempo } \\
\hline & $\begin{array}{l}\text { Present } \\
\text { study }\end{array}$ & $\begin{array}{l}\text { Other } \\
\text { studies }\end{array}$ & $\begin{array}{l}\text { Present } \\
\text { study }\end{array}$ & $\begin{array}{l}\text { Other } \\
\text { studies }\end{array}$ & $\begin{array}{l}\text { Present } \\
\text { study }\end{array}$ & $\begin{array}{l}\text { Other } \\
\text { studies }\end{array}$ & $\begin{array}{l}\text { Present } \\
\text { study }\end{array}$ & $\begin{array}{l}\text { Other } \\
\text { studies }\end{array}$ \\
\hline Neutral & - & $\pm(10)$ & - & $-(10)$ & \pm & $?$ & \pm & $+(10)$ \\
\hline Disgust & - & $-(7,11)$ & \pm & $-(11)$ & + & $\pm(7)$ & - & $-(11)$ \\
\hline Surprise & + & $+(11)$ & + & $+(5,11)$ & + & $?$ & \pm & $+(11)$ \\
\hline Shame & - & $?$ & - & $?$ & - & $?$ & - & $?$ \\
\hline Interest & + & $?$ & + & $+(11)$ & \pm & $?$ & + & $+(11)$ \\
\hline Joy & + & $+(2,5,6,8,9,11)$ & + & $+(5,11)$ & + & $+(2,6$ & + & $+(2,11)$ \\
\hline Fear & + & $\begin{array}{l}+(4,10) \\
\pm(5 a) \\
-(5 b)\end{array}$ & \pm & $\begin{array}{l}+(4,10,11) \\
-(5,11)\end{array}$ & \pm & $?$ & + & $+(3,10,11)$ \\
\hline Contempt & - & $-(4,5 a)$ & + & $\begin{array}{l}+(4) \\
-(5)\end{array}$ & + & $?$ & - & $-(3)$ \\
\hline Sadness & + & $\begin{array}{l} \pm(9) \\
-(2,4,6,7,8,10)\end{array}$ & - & $\begin{array}{l}-(4,11) \\
\pm(10)\end{array}$ & - & $-(2,6,7)$ & - & $-(2,3,10,11)$ \\
\hline Anger & + & $\begin{array}{l}+(2,4) \\
\pm(5 a, 10)\end{array}$ & + & $+(4,10)$ & + & $+(1,2)$ & + & $+(1,2,3,10,11)$ \\
\hline
\end{tabular}

Note. $\quad 5=$ Hungarian, German, English, French, $5 a=$ Hungarian, $5 b=$ German, English, French $+=$ hıgh, wide, loud, fast, $\pm=$ mid, $-=$ low, narrow, solt, slow 
Table 37 Survey of studies on the characteristics of vocal expressions of emotion

\begin{tabular}{|c|c|c|c|c|c|c|c|}
\hline Number & Study & Encoders & Induction & Speech sample & Emotions & Language & Description \\
\hline 1 & Bortz, 1966 & 5 males & sımulation & nonsense word & anger, neutral & German & Instrumental \\
\hline 2 & Davitz, $1964 b$ & ? & simulation & standard passage & $\begin{array}{l}\text { joy, sadness, } \\
\text { anger }\end{array}$ & Am English & perceptual \\
\hline 3 & $\begin{array}{l}\text { Faurbanks \& } \\
\text { Hoaglın, 1941 }\end{array}$ & $\begin{array}{l}6 \text { males } \\
\text { (actors) }\end{array}$ & simulation & standard passage & $\begin{array}{l}\text { fear, contempt, } \\
\text { sadness, anger }\end{array}$ & Am Englısh & Instrumental \\
\hline 4 & $\begin{array}{l}\text { Fairbanks \& } \\
\text { Pronovost, } 1939\end{array}$ & $\begin{array}{l}6 \text { males } \\
\text { (actors) }\end{array}$ & simulation & standard passage & $\begin{array}{l}\text { fear, contempt, } \\
\text { sadness, anger }\end{array}$ & Am Englısh & instrumental \\
\hline 5 & $\begin{array}{l}\text { Fónagy \& } \\
\text { Magdics, } 1963\end{array}$ & $?$ & $\begin{array}{l}\text { simulation/ } \\
\text { spontaneous }\end{array}$ & $\begin{array}{l}\text { excerpts from } \\
\text { conversations, } \\
\text { theaterplays, etc }\end{array}$ & $\begin{array}{l}\text { surprise, Joy, fear, } \\
\text { contempt, anger }\end{array}$ & $\begin{array}{l}\text { Hungarian, } \\
\text { German, } \\
\text { Englısh, } \\
\text { French }\end{array}$ & perceptual \\
\hline 6 & Huttar, 1968 & 1 male & spontaneous & $\begin{array}{l}\text { selected } \\
\text { utterances }\end{array}$ & $\begin{array}{l}\text { Joy, fear, sadness, } \\
\text { anger }\end{array}$ & Am English & $\begin{array}{l}\text { instrumental/ } \\
\text { perceptual }\end{array}$ \\
\hline 7 & Ka1ser, 1962 & $\begin{array}{l}3 \text { males and } \\
7 \text { females }\end{array}$ & sımulation & "a", "o", "e" & disgust, sadness & Dutch & instrumental \\
\hline 8 & $\begin{array}{l}\text { Sedlácek \& } \\
\text { Sychra, } 1963\end{array}$ & $\begin{array}{l}23 \text { females } \\
\text { (actrices) }\end{array}$ & $\begin{array}{l}\text { mood induction/ } \\
\text { simulation }\end{array}$ & standard sentence & $\begin{array}{l}\text { joy, fear, } \\
\text { sadness }\end{array}$ & Czech & instrumental \\
\hline 9 & Skınner, 1935 & $\begin{array}{l}9 \text { males and } \\
10 \text { remales } \\
\text { (drama students) }\end{array}$ & mood induction & “ah" & joy, sadness & Am English & instrumental \\
\hline 10 & $\begin{array}{l}\text { Williams \& } \\
\text { Stevens, } 1972\end{array}$ & $\begin{array}{l}3 \text { males } \\
\text { (actors) }\end{array}$ & playactıng & standard passage & $\begin{array}{l}\text { fear, sadness, } \\
\text { anger, neutral }\end{array}$ & Am English & instrumental \\
\hline 11 & Scherer, 1974 & - & - & $\begin{array}{l}\text { synthesized tone } \\
\text { sequences }\end{array}$ & $\begin{array}{l}\text { disgust, surprise, } \\
\text { interest, joy, fear, } \\
\text { sadness, anger }\end{array}$ & - & instrumental \\
\hline
\end{tabular}


In Table 3.6 the results of the present study, obtained by means of the categorization procedure mentioned above, and those available from other studies, either taken verbatim from the original study or categorized according to our own views, are presented with regard to pitch level, pitch range, loudness (or vocal effort), and tempo. In view of differences in experimental design, a brief summary of the characteristics of the studies from which results have been used is given in Table 3.7. In this table only those emotions are presented which coincide with the ones investigated in the present study.

It appears from Table 3.6 that researchers have concentrated on gathering information on the characteristics of only a few emotions, namely joy, fear, sadness, and anger. Neutral, disgust, surprise, shame, interest, and contempt have hardly been looked at. Neutral occupies a special position in this respect. It has been included in studies fairly often, but mostly to function as a point of reference for the other - emotional - categories examined; its characteristics are rarely explicitly reported.

There is, however, another type of research in which neutral speech has been considered, not in contrast with other - emotional - categories individually, but rather in contrast with them as a group, i.e., in the sense of non-emotional versus emotional. This type of research aims at determining the vocal characteristics of speech produced in a situation of stress, i.e., a situation in which the balance of the organism has been upset by some kind of disturbing event. Stress has been shown to involve a series of physiological and behavioural changes, characterized by some degree of activation or emotional tension, which prepare the organism for an appropriate response.

Scherer (1981a) summarizes the findings of empirical studies on vocal correlates of stress, both in real-life situations and in a laboratory setting. $\mathrm{He}$ concludes that "the vocal parameters which seem most likely to qualify as indicators of stress are [an increase in] fundamental frequency and energy concentration between 500 and $1000 \mathrm{~Hz}$, possibly due to tense voice" (p. 179). From this it may be inferred that "non-stressed" or neutral speech is characterized by a relatively low fundamental frequency and great laryngeal laxness. These outcomes agree with ours (see Table 3.4). Scherer explains the characteristics of stress - very cautiously - as a result of increased subglottal pressure, higher medial compression, and tension of the vocal folds, phenomena which in their turn would result from the deepening of respiration, the delation of the bronchi, and the increase in muscle tone under stress.

Going back to Table 3.6, it may be seen that there are not enough data on the characteristics of shame and interest to allow any statement as to possible cross-cultural differences or similarities. The findings for the other emotions agree fairly well, except for sadness, which was found to have a high pitch level in the present study whereas in all other studies to date it was found to have a low or intermediate pitch level. We have no explanation for this discrepancy.

In general, the degree of convergence of the results may look quite impres- 
sive, especially if one considers the differences in experimental setup of the studies from which they stem and the lack of criteria for, and therefore the probable inconsistency of, the categorization. If, however, one takes into account the fact that the research carried out to date pertains to few and rather closely connected cultures, only some European languages being represented, and the fact that only a restricted number of discrete emotions have been more or less systematically studied, it is clear that much more descriptive research needs to be conducted in many more cultures and with regard to many more emotions before more definite statements as to the universality of vocal pattems of emotion can be made. Especially descriptions of emotional utterances coming from "auditorily isolated" cultures, i.e., cultures with few opportunities to hear Westerners speak, would be very informative. Also, the need for detailed descriptions covering a wide range of parameters, preferably reported numerically and not verbally, cannot be stressed enough.

The importance of the latter remark may be illustrated with an example. On the basis of the data with respect to the four parameters presented in Table 3.6 it would not be possible to differentiate between the vocal patterns of joy and anger: Both are characterized by a high pitch level, a wide pitch range, a loud voice, and a fast tempo. Now it could be hypothesized that fairly excessive - expressions of joy and anger are highly similar. This could mean that their discrimination would rely very heavily on information provided via the verbal channel and on extralinguistic sources of information such as the situational context and the facial expression and posture of the speaker. This hypothesis can be tested by inspecting the results of recognition experiments in which the emotional expressions are carried by standard speech material and in which no extralinguistic information is available. If the expressions of joy and anger do indeed hardly differ, subjects will have a hard time keeping them apart, which will result in a great number of mutual confusions. And indeed, confusions between joy and anger have occasionally been reported, cross-culturally (Albas, McCluskey, \& Albas, 1976) as well as intra-culturally (Dimitrovsky, 1964). However, the confusions are not frequent enough to warrant the conclusion of a complete absence of any differences.

It could be that one or more of the four parameters presented in Table 3.6 do contain information which allows the listener to differentiate expressions of joy from expressions of anger, but that the three categories distinguished (high, mid, low, etc.) are too broad to reveal where the differences lie. There may be slight but consistent differences in pitch level, for example. (The data presented in Table 3.4 seem, indeed, to point in this direction.) Of course, these differences only show up in a detailed report, preferably numerical, of the data.

Another possibility, which does not exclude the preceding one, is that expressions of joy and anger differ with respect to some parameter(s) which is (are) not commonly examined. For our own material it appears, for example, 
that joy tends to be characterized by lip spreading, while anger is not. These and other data are bound to remain undetected unless comprehensive and detailed descriptions are available, preferably based on a well-documented system such as the one developed by Laver. The use of a standard system would also greatly enhance the comparability of the descriptive findings, and stimulate research. This has been demonstrated by the impetus given to the study of facial expressions of emotion by the recent development of comprehensive facial measurement techniques, such as Ekman and Friesen's FACS (Facial Action Coding System) (1978). Of course, acoustic descriptions may be very useful too. Acoustic measures have the advantage of being objective and well-definable. However, the value of acoustic data largely depends on what is known about their relationship to perception or physiology. This problem will be treated in more detail in Chapter 5 . 



\section{Relative importance of perceptual parameters and the characteristics of emotional dimensions}

\subsection{INTRODUCTION}

In Chapter 3 the results were reported of a number of analyses of variance and a posteriori contrast tests based on the auditory ratings of 160 emotional utterances. From these it appeared that at least twelve vocal parameters may be relevant to distinguishing the ten emotional categories examined. This assumption seems even more plausible as in general the correlations between the ratings on the different parameters were not very high. It is not likely, however, that all parameters would be equally important, nor is it necessarily true that the four parameters on which most knowledge has been gathered to date, namely pitch level, pitch range, tempo, and loudness, are also the most important ones for communicating emotional meaning.

In the present chapter an attempt will be made to establish the relative importance and specific contributions of the twelve vocal parameters to the statistical - discrimination among the ten emotions under study by examining the outcomes of a multiple discriminant analysis (Cooley \& Lohnes, 1971). In view of the nature and the possibilities of the analysis used emphasis will not be so much on the contribution of the various parameters to the identification of individual emotional categories, but rather on their contribution to dimensions of emotional meaning.

Little research has been conducted into the relative importance of different vocal features for the discrimination among vocal expressions of emotion. A few researchers have tried to gain insight into the problem by comparing the recognition of unprocessed emotional utterances with the recognition of the same stimuli but with part of the acoustic information removed. This approach was adopted by Lieberman and Michaels (1962), Pollack, Rubenstein, and Horowitz (1960), and Ross, Duffy, Cooker, and Sargeant (1973).

Lieberman and Michaels (1962) derived pitch and amplitude information from simulated vocal expressions of boredom, confidence, disbelief or doubt, fear, happiness, a question, an objective statement, and a pompous statement, produced by three adult males, and used that information to control a fixed-vowel synthesizer. The aim of the study was to examine the relative contributions of amplitude (an acoustic correlate of perceived loudness) and pitch $^{1}$ on emotion recognition. The stimuli were presented in 
six different versions, one non-manipulated and five manipulated, to separate groups of subjects for identification.

It appeared that with the non-manipulated speech the emotional and attitudinal (or syntactic) categories were correctly identified $85 \%$ of the time. With only pitch and amplitude information (articulatory and voice quality information removed) recognition dropped to $47 \%$. With only pitch information there was a slight additional drop of $3 \%$. Retaining amplitude information, but smoothing the pitch curve with a 40 ms-time constant reduced the recognition rate to $58 \%$, and $100 \mathrm{~ms}$ smoothing to $25 \%$. A 120 Mz monotone with amplitude information resulted in $14 \%$ correct responses, a score which still exceeded chance expectancy at a level of significance beyond $1 \%$.

These outcomes suggest that articulatory and voice quality information plus - and perhaps most importantly - the transition from speech to remotely speech-like stimuli, gross pitch movements (including pitch range), small pitch variations, and amplitude contributed to the transmission of emotional and attitudinal meaning in that order. It may also be concluded that a great deal of acoustic information can be removed before recognition rate drops below chance. A final finding worth mentioning is that not all categories examined depended on the various acoustic parameters to the same degree: The identification of the category question, for example, depended strongly on pitch information (final rise?), whereas the category boredom was highly resistant to confusions under all conditions (because the boredom expressions had little pitch and amplitude variation to start with?).

The finding that fairly high levels of recognition may be achieved with sharply reduced acoustical information is confirmed in the results of a study conducted by Pollack, Rubenstein, and Horowitz (1960), who compared the recognition of simulated expressions in normal and whispered (i.e., practically non-periodic) speech under varying $\mathrm{S} / \mathrm{N}$ ratios, and a study by Ross, Duffy, Cooker, and Sargeant (1973), who compared recognition of simulated emotional expressions in their original form and several filtered conditions. However, the way in which these studies have been designed does not permit any statements as to the relative importance of the various cues examined.

Scherer, Koivumaki, and Rosenthal (1972) presented vocal expressions of emotion taken from a recorded version of a play to be rated on twenty semantic differential scales representing the three major dimensions of meaning found by Osgood, Suci, and Tannenbaum (1957), i.e., activity, evaluation, and potency. The expressions were offered in four different conditions. In one condition the samples were in their original form; in the other three they were content masked by means of lowpass filtering (cut-off frequency $650 \mathrm{~Hz}$ ), random splicing, and a combination of both techniques. The mean correlation (averaged over the twenty rating variables) between the ratings of the non-manipulated samples and the spliced-plus-filtered samples was as high as .91. From these findings Scherer et al. conclude that those - few - cues 
that remain largely unaffected by both filtering and splicing, i.e., pitch level and pitch range, loudness level and loudness range, and rate of articulation constitute all, or at least an important part, of the minimal set of vocal cues communicating basic emotional meaning and that sequential speech variables (such as pitch contour, rhythm, and pauses) and formant cues are of minor importance.

More insightful is the study by Scherer and Oshinsky (1977). They synthesized tone sequences in which amplitude range, pitch level, pitch contour, pitch range, tempo, envelope (different attack/decay ratios, round envelope being comparable to drawl or slurred speech and sharp envelope to clipped speech), and cut-off point (number of harmonics filtered out, few harmonics being closer to resonant voice and many harmonics being closer to blaring or shrill voice) were independently varied. The stimuli were rated on three semantic differential scales, namely, active/passive, pleasant/unpleasant, and strong/weak. The results of a multiple regression analysis showed that about two-thirds to three-quarters of the variance in the semantic differential ratings could be accounted for by the manipulations of the acoustic parameters, and that a linear model of the judges' cue utilization seemed to be a reasonably good approximation of their response system. On the whole, tempo appeared to be the most powerful predictor: It accounted for $31 \%$ of the variance compared to $14 \%$ for the number of harmonics, and between $3 \%$ and $5 \%$ for the remaining acoustic parameters (except for amplitude range: less than $1 \%$ ). However, the rank order differed for each of the three scales.

Bortz (1966) also conducted a correlational study. He examined the relationship between fourteen acoustic variables, derived from the output of a third octave filter bank, and the ratings on twelve semantic differential scales. The data base consisted of the expressions of five different emotions with a nonsense word. It appeared that $92 \%$ of the variance in the ratings could be accounted for by the combined acoustic measures. A canonical correlation revealed that the factor intensity was the most powerful predictor and the factor rhythm the second most powerful. Both factors correlated about equally highly with the activity and evaluative dimensions emerging from the ratings.

From the above survey at least one conclusion may be drawn, namely that the vocal cues that are utilized for the inference of emotional meaning or ratings on semantic differential scales must be very redundant: Whichever (groups of) cues are removed, the attribution of emotional meaning almost always remains possible. The question of the relative importance of (sets of) cues, however, is far from being resolved, almost every study yielding different results, probably also as a consequence of the use of widely varying research methodologies and speech samples. Thus, obviously more studies on the relative importance of vocal cues are badly needed. 


\subsection{METHOD}

\subsubsection{Input data}

The input to the discriminant program (SPSS program DISCRIMINANT [Klecka, 1975]) were the ratings averaged over 6 raters for each of 160 emotional utterances on each of 12 vocal parameters. The 12 parameters were lip spreading, laryngeal tension, laryngeal laxness, creak, tremulousness, whisper, harshness, pitch level, pitch range, loudness, tempo, and precision of articulation. The 160 utterances consisted of 8 speakers ( 4 males and 4 females) $\times 2$ phrases (twee maanden zwanger and zo'n grote Amerikaanse auto) $\times 10$ emotions (neut ral [NE], disgust [DI], surprise [SU], shame [SH], interest[IN], joy [JO], fear [FE], contempt [CO], sadness [SA], and anger [AN]). (For a detailed description of the rating procedure, see Chapter 3; for a detailed description of the speech material, see Chapter 2.)

\subsubsection{Analysis ${ }^{2}$}

The ultimate statistical goal of multiple discriminant analysis is to optimally separate groups of cases (in the present study: ten emotional categories each comprising sixteen utterances) by forming one or more linear and weighted combinations of discriminating - or rather potentially discriminating - variables which measure characteristics on which the groups are expected to differ (in the present study: twelve vocal parameters). The first discriminant function is that single weighted composite that provides maximum average separation between the groups relative to the variability within the groups. The second discriminant function is that weighted composite which of all possible weighted composites uncorrelated with the first provides maximum average separation between the groups, and so on. The maximum number of potential discriminant functions is equal to the number of discriminating variables or to one less than the number of groups, whichever is smaller.

Standarized discriminating functions are of the form:

$$
D_{1}=d_{11} Z_{1}+d_{12} Z_{2}+\ldots+d_{1 p} Z_{p}
$$

where $D_{1}$ is the score on discriminant function $i$, the $d$ 's are weighting coefficients (or standardized discriminant function weights) and the $Z$ s are the standardized values of the $p$ discriminating variables used in the analysis. Thus, the discriminant score is computed by multiplying each discriminating variable by its corresponding coefficient and adding together the products. There will be a separate score for each case on each function. For a single group, the means on all functions are referred to as the group centroid, which is the most typical location of a case from that group in the discriminant function space. A comparison of the group means on each function indicates how far apart the groups are along that dimension. The adequacy of the 
discriminant functions may be tested by having the cases classified that were used to derive the functions in the first place and comparing predicted group membership with actual group membership.

\subsection{RESULTS}

First, we will present the optimal set of discriminating variables as it appeared from a number of step-wise procedures. Next, we will examine the contributions of the various parameters to separating the emotional categories by inspecting the composition of the functions extracted, and specifically the standardized weights. Further insight into the functions will be gained by examining the locations of the group centroids in the discriminant space. Finally, the relations among the emotions will also be approached by means of a hierarchical cluster analysis carried out on the classification results.

A direct test of the homogeneity of group dispersions, a condition that the data must formally fulfill in order to be amenable to multiple discriminant analysis, was made by using a generalized test of homogeneity of variance. This analysis tests the differences in dispersion between groups using what is commonly referred to as Box's HI statistic. The result obtained for this test using all twelve parameters was $M=1802, F(546,16281.6)=2.16, p<.01$. For the lower ordered variable solutions Box's $\mathrm{Hl}$ was significant at the $1 \%$ level also. From these outcomes it may be inferred that our data do not satisfy the condition of homogeneous group dispersions. However, in practice, multiple discriminant analysis is a very robust technique, and this assumption need not be strongly adhered to (Klecka, 1975, p. 435 , note 2). Therefore, we will now proceed, be it with caution, to the presentation of the actual results.

\subsubsection{Classification}

One way of obtaining an estimate of the relative importance of the different parameters for separating the emotional categories is to use a step-wise procedure, i.e., a method in which the discriminating variables are selected for entry into the analysis on the basis of their discriminating power. The rationale behind choosing a step-wise selection method instead of the direct method, in which all variables are entered into the analysis concurrently, is that in many cases the full set of discriminating variables contains redundant information about the differences among the groups; thus, it is often possible to find a reduced set of variables which is almost as good as, and sometimes even better than, the full set.

The step-wise procedure may be described as follows: At the first step the single most powerful variable is chosen, according to a pre-established selection criterion. This initial variable is then paired with each of the other variables. The variable that in combination with the initial variable produces 
the best criterion value is the second to enter the analysis. This process continues till all variables have been entered, or until no additional variables provide a minimum level of improvement. Cut-off values may be specified to limit the number of variables to be inserted.

We will present the results of six step-wise analysis runs: One in which the discrimination was based on the one, two, three, four, and five most powerful variables, and one in which the full set of twelve discriminating variables was inserted. The results of the six, seven, eight, nine, ten, and eleven variable solutions were all very similar to those of the five and twelve variable solutions, and will therefore not be presented.

Table 4.1 summarizes part of the results of the analyses, namely for each analysis run the number of utterances per group that were classified correctly, the total number of utterances assigned to each group, and the percentages of variance explained by the discriminating functions. In the one, two, and three variable solutions the maximum number of functions has been derived equaling the number of discriminating variables; in the higher ordered solutions only the first three functions have been retained. This has

Table 4.1 Number of emotional utterances correctly classified (maxımum score $=16$ ) and assigned to each group (in parentheses) in six discriminant analysis runs with an increasing number of perceptually based discrimınating variables. Order of insertion: loudness, laryngeal laxness, pitch level, pitch range, laryngeal tension, harshness, tempo, creak, tremulousness, up spreading, precision of articulation, whisper

\begin{tabular}{|c|c|c|c|c|c|c|}
\hline & \multicolumn{6}{|c|}{ Number of variables } \\
\hline & 1 & 2 & 3 & 4 & 5 & 12 \\
\hline Neutral & $10(19)$ & $12(20)$ & $12(19)$ & $13(18)$ & $15(20)$ & $16(20)$ \\
\hline Disgust & $1(10)$ & $S(23)$ & $7(13)$ & $7(17)$ & $8(17)$ & $9(17)$ \\
\hline Surprise & $2(19)$ & $2(19)$ & $2(16)$ & $8(13)$ & $7(17)$ & $6(15)$ \\
\hline Shame & $14(25)$ & $8(9)$ & $9(10)$ & $9(10)$ & $10(11)$ & $11(13)$ \\
\hline Interest & $1(7)$ & $7(17)$ & $9(17)$ & $10(15)$ & $13(19)$ & $14(18)$ \\
\hline Joy & $5(20)$ & $5(20)$ & $12(22)$ & $12(24)$ & $6(13)$ & $7(16)$ \\
\hline Fear & $0(0)$ & $2(6)$ & $6(12)$ & $10(20)$ & $12(18)$ & $10(16)$ \\
\hline Contempt & $4(20)$ & $1(1)$ & $4(11)$ & $2(9)$ & $5(9)$ & $5(9)$ \\
\hline Sadness & $3(21)$ & $8(26)$ & $9(24)$ & $9(18)$ & $10(17)$ & $10(17)$ \\
\hline Anger & $11(19)$ & $11(19)$ & $13(16)$ & $12(17)$ & $12(19)$ & $12(19)$ \\
\hline Mean (in \%) & 32 & 38 & 52 & 58 & 61 & 62 \\
\hline \multicolumn{7}{|l|}{$\begin{array}{l}\text { \% variance } \\
\text { accounted } \\
\text { for by }\end{array}$} \\
\hline Function I & 100 & 70 & 55 & 52 & 48 & 41 \\
\hline Function II & - & 30 & 27 & 22 & 24 & 22 \\
\hline Function III & - & - & 18 & 18 & 17 & 18 \\
\hline $\begin{array}{l}\text { Total of three } \\
\text { functions }\end{array}$ & 100 & 100 & 100 & 92 & 89 & 81 \\
\hline
\end{tabular}


mainly been done because the fourth and further functions, in addition to adding only a relatively small percentage of explained variance, proved to be impossible to interpret, a finding which is commonly reported in the literature (cf. Cooley \& Lohnes, 1971, p. 244).

Table 4.1 clearly shows that from the one variable solution up to the four variable solution there is a considerable and consistent progression in the overall percentage of correctly classified utterances when discriminating variables are added. However, a binomial estimate of probability revealed that even the $31 \%$ obtained in the one variable solution is far beyond chance $(p<.01)$. From the five variable solution on, adding more variables does not seem to make much of a difference in terms of overall accuracy. This means that on the whole loudness, laryngeal laxness, pitch level, pitch range, and laryngeal tension succeed in separating the utterances belonging to the various emotional categories virtually just as well as the complete set of twelve variables.

It may also be seen from Table 4.1 that the total number of utterances that are - justly or unjustly - assigned to each group (indicated in parentheses) is rather variable: Surprise and anger are the only two groups that receive fairly regularly in all six solutions approximately the sixteen utterances that one would expect; the number of utterances assigned to disgust, shame, interest, joy, fear, and contempt varies drastically from one analysis run to the other, whereas neutral and sadness consistently receive more than their due share. The total number of utterances assigned to a group should really be taken into account when examining the numbers of correct assignments.

A closer look at the data presented in Table 4.1 reveals that in the one variable solution, i.e., with loudness as the sole discriminating variable, expressions of shame and anger, and to a lesser degree those of neutral, are classified remarkably well, even if one takes the positive response biases into account (cf. the values given in parentheses). Inspection of the mean scores and standard deviations given in Table 3.4 may help to clarify this outcome. It appears that the mean loudness scores for shame and anger are respectively by far the lowest and by far the highest of all categories, with a moderate standard deviation. The mean for neutral is less extreme but then the standard deviation is exceptionally small. These data explain why, only on the basis of loudness information, these three categories have been so easy to separate from the rest.

The insertion of laryngeal laxness in the two variable solution makes no or little difference for the classification of neutral, surprise, joy, fear, contempt, and anger expressions. However, almost half of the utterances that were justly assigned to shame in the one variable solution have now been removed to be unjustly assigned to other categories: three to neutral, two to sadness, and one to interest. On the other hand, it may be observed that it is not only the number of correctly classified shame utterances that has dropped considerably, but also the number of utterances that previously were incorrectly grouped with shame. The classification of disgust, interest, and sadness 
utterances may be seen to have been affected by the insertion of laryngeal laxness in a positive way (note, however, the simultaneous increase in the total number of utterances assigned to these categories).

The insertion of pitch level in the three variable solution has a positive effect on the categorization of all categories (except neutral), but especially on that of joy and fear. The data in Table 3.4 reveal that these two emotions had the highest mean pitch levels of all categories, with a medium standard deviation.

The most striking effect of the insertion of pitch range in the four variable solution is that it enhances considerably the number of correctly classified surprise utterances. This is by no means surprising, since the mean pitch range score for this emotion lies far beyond the scores obtained for the others (Table 3.4).

Although the insertion of the fifth variable, that is, laryngeal tension, hardly affects the overall correct classification, its effect on the number of utterances that are - correctly or incorrectly - assigned to the category joy is quite dramatic. In the four variable solution the group of presumed joy utterances is a somewhat broad and fuzzy amalgam of emotions, the number of which is almost reduced to half by the introduction of laryngeal tension in the five variable solution. From the eleven utterances taken away from joy there are six that were correctly classified; three of these are now confused with anger, two with surprise, and one with interest.

As was stated above, the insertion of the higher ordered variables, i.e., harshness, tempo, creak, tremulousness, lip spreading, precision of articulation, and whisper, has only a minor effect on the classification results, not only with respect to overall accuracy but also with respect to the correct classification of the representatives of individual groups, and will therefore not be further gone into.

\subsubsection{Dimensions}

As well as by examining the outcomes of the various step-wise analysis runs, insight into the relative importance of the various parameters may also be gained by looking at the standardized discriminant function weights (the $d$ 's in the formula given above) in the twelve variable solution. When their sign is ignored, each coefficient represents the relative contribution of its associated variable to that function. The sign merely denotes whether the variable makes a positive or a negative contribution. Of course, since the functions are arranged in order of decreasing importance, a given contribution to the third function is not as important in terms of its effect on overall separation as the same contribution to the first function. As in factor analysis, the coefficients may be used to name the functions by identifying the dominant characteristics they measure. In Table 4.2 the standardized weights for the first three functions extracted from the analysis run with the full set of twelve discriminating variables are presented. 
Table 4.2 Discriminant analysis with twelve perceptually based discriminatıng varıables: Standardized discriminant function weights for the first three functions extracted

\begin{tabular}{lrrr}
\hline & \multicolumn{2}{c}{ Functions } & \\
\cline { 2 - 4 } & \multicolumn{1}{c}{1} & \multicolumn{1}{c}{3} \\
\hline Pitch level & -0.20 & 0.09 & 1.04 \\
Pitch range & 0.52 & 0.30 & 0.11 \\
Loudness & 0.40 & 0.70 & -0.37 \\
Tempo & 0.04 & 0.20 & 0.37 \\
Precision of articulation & -0.15 & 0.22 & -0.02 \\
Lip spreading & 0.14 & -0.08 & 0.02 \\
Laryngeal tension & 0.75 & -0.67 & -0.43 \\
Laryngeal laxness & -0.26 & 0.50 & -0.02 \\
Creak & -0.12 & -0.23 & 0.12 \\
Tremulousness & 0.08 & -0.20 & 0.18 \\
Whisper & -0.01 & 0.20 & 0.04 \\
Harshness & -0.44 & 0.22 & 0.63 \\
\hline
\end{tabular}

It may be seen from Table 4.2 that the first function is characterized by a high positive contribution of laryngeal tension, fairly high positive contributions of pitch range and loudness, and a fairly high negative contribution of harshness. The second function appears to have a high positive contribution of loudness, a high negative contribution of laryngeal tension, and a fairly high positive contribution of laryngeal laxness. The third function has a very high positive contribution of pitch level, a high positive contribution of harshness, and a fairly high negative contribution of laryngeal tension.

Thus, it appears that the composition of the various functions may be largely described in terms of different combinations of loudness, laryngeal tension, laryngeal laxness, and harshness, complemented with positive contributions of pitch range and pitch level. The other variables, namely, tempo, precision of articulation, lip spreading, creak, tremulousness, and whisper, hardly play a role in the formation of any of the three functions. These findings largely corroborate the outcomes obtained with the various step-wise analysis runs given above, where loudness, laryngeal laxness, pitch level, pitch range, and laryngeal tension also appeared to be the best set of discriminating variables. However, the contribution of harshness to the functions appears to be more considerable than one would have expected on the basis of its sixth rank order position in the insertion of variables in the step-wise procedure.

The fact that several variables contribute substantially to more than one function, the fact that a number of variables that correlate positively with each other, such as loudness and laryngeal tension (see Table 3.3), sometimes make opposite contributions, and the fact that, except for pitch level with respect to the third function, the relative heights of the contributions of the 
different variables are not very far apart, make it difficult to interpret the functions and give them a name.

The first function, which is mainly characterized by positive contributions of laryngeal tension and pitch range, suggests an interpretation in terms of level of activity. The second function could be related to activity as well, although the about equally high but opposite contributions of loudness and laryngeal tension are somewhat confusing in this respect. It is not implausible that the third function, with its dominant positive contribution of pitch level, may also have something to do with activity. Thus, the contributions of the variables to the functions suggest that all three may be interpreted in terms of different kinds of activity.

An alternative approach to interpreting and naming the functions is to study the values of the group means, i.e., the projections of the group centroids on the dimensions in the discriminant space. The discriminant space spanned by the first and second dimensions is depicted graphically in Figure 4.1; in Figure 4.2 the space spanned by the first and third dimensions is shown.

The positions of the different emotions on the first dimension presented in Figure 4.1 clearly suggest that this dimension be called an activity dimension, shame, neutral, and sadness, the three emotions situated on the left, being the most passive emotions, and surprise and anger, situated on the right, being the most active ones. Thus, the impression formed on the basis of the contributions of the variables to this dimension is confirmed.

The second dimension presented in Figure 4.1 extends from sadness and

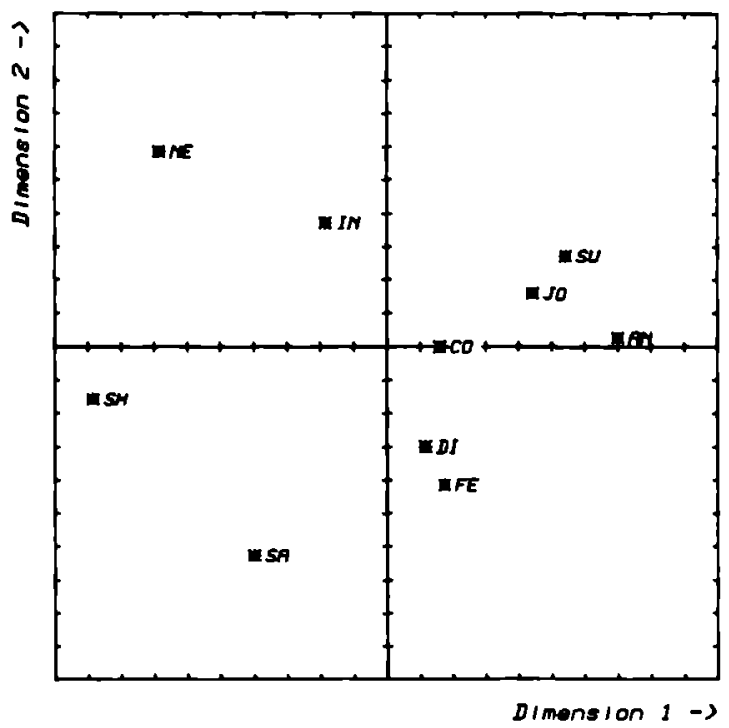

Figure 4. I Positions of ten emotions along the first and second dimensions resulting from a discriminant analysis based on the perceptual ratings for twelve vocal parameters 


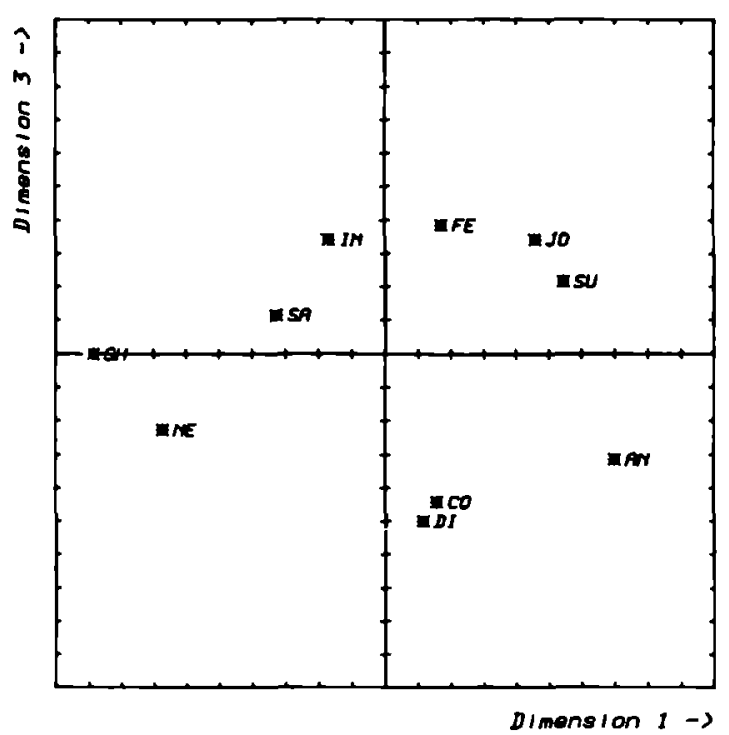

Figure 42 Positions of ten emotions along the first and third dimensions resulting from a discriminant analysis based on the perceptual ratıngs for twelve vocal parameters

fear at the one extreme via contempt and anger in the middle to neutral at the other extreme. This dimension, which was more difficult than the first to interpret in terms of the weightings of the variables, is not easy to interpret in terms of a rank ordenng of the emotions either If it were not for the extreme position of neutral and the central position of anger, it could be interpreted as an evaluative dimension, having "bad" emotions like sadness and fear at one end and the "good" emotions of joy and interest at the other For this dimension we should perhaps refrain from giving a semantic interpretation and content ourselves with stating that it runs from tense and soft to lax and loud.

The third dimension, presented in Figure 4.2, with disgust and contempt at the one extreme, shame in the middle, and interest, joy, and fear at the other extreme, we did not succeed in giving a semantic interpretation either; its dominant characterıstıc is, in any case, pitch level.

\section{Confusions}

In a final attempt to gain insight into the relationships among the emotions under study we decided to examine the classification results of the discriminant analysis based on the total set of twelve parameters and, more specifically, to have a closer look at the confusions that were made. The classification results are presented in Table 43 in the form of a confusion 
Table 4.3 Classification results of a discriminant analysis with twelve perceptually based discnminating varıables Order of insertion. loudness, laryngeal laxness, pitch level, pitch range, laryngeal tension, harshness, tempo, creak, tremulousness, lip spreading, precision of articulation, whisper Maximum score $=16$

\begin{tabular}{lrrrrrrrrrrr} 
Actual group & \multicolumn{10}{l}{ Predicted group membership } \\
\cline { 2 - 11 } & NE & DI & SU & SH & IN & JO & FE & CO & SA & AN \\
\hline Neutral & 16 & 0 & 0 & 0 & 0 & 0 & 0 & 0 & 0 & 0 \\
Disgust & 0 & 9 & 1 & 1 & 0 & 0 & 0 & 3 & 1 & 1 \\
Surprise & 0 & 0 & 6 & 0 & 2 & 5 & 0 & 0 & 0 & 3 \\
Shame & 2 & 0 & 0 & 11 & 0 & 0 & 0 & 0 & 3 & 0 \\
Interest & 0 & 0 & 0 & 0 & 14 & 1 & 1 & 0 & 0 & 0 \\
Joy & 0 & 0 & 4 & 0 & 2 & 7 & 2 & 0 & 0 & 1 \\
Fear & 0 & 0 & 0 & 0 & 0 & 3 & 10 & 0 & 3 & 0 \\
Contempt & 2 & 4 & 2 & 0 & 0 & 0 & 1 & 5 & 0 & 2 \\
Sadness & 0 & 3 & 0 & 1 & 0 & 0 & 2 & 0 & 10 & 0 \\
Anger & 0 & 1 & 2 & 0 & 0 & 0 & 0 & 1 & 0 & 12 \\
\hline
\end{tabular}

matrix. On the diagonal the number of correctly classified utterances (corresponding with the last column in Table 4.1) are given for each group:, in the off-diagonal cells the confusions may be found.

The data given in the confusion matrix presented in Table 4.3 make clear that not all emotions have been confused with one another equally often. Of course, this is what one would expect, since in the discriminant space (see Figures 4.1 and 4.2) not all groups lie equally close together. On the basis of the raw confusion data, however, it is not easy to detect what exactly the patterns of confusions are. A technique which is particularly well suited to provide insight into which groups of confused emotions may be distinguished is hierarchical cluster analysis (Johnson, 1967). Thus, this technique may be considered as another means of getting an idea of the distances between the emotions under study, and, indirectly, of the structure of the emotional space.

Since hierarchical cluster analysis cannot handle asymmetrical confusions, i.e., cannot deal with cases in which emotion $i$ is mistaken for emotion $j$ more often than emotion $j$ is mistaken for emotion $i$, the confusion matrix presented in Table 4.3 was first made symmetrical, according to the method described by Klein, Plomp, and Pols (1970), before the analysis was carried out.

Hierarchical cluster analysis provides at least two methods for defining clusters, one, the so-called minimum or nearest neighbour method, favouring the formation of new clusters and the other, the so-called maximum or furthest neighbour method, favouring the addition of elements to clusters already formed. Both methods were applied. It appeared that the correlation between the distances in the data matrix and the ultrametric distances was higher for the maximum method than for the minimum method (.78 and .65 , 
respectively), which means that the former represents the original data better than the latter. On the other hand, the structure of the dendrogram resulting from the application of the minimum method, consisting of three clearly distinguished clusters plus neutral, appeared to be more insightful than the structure of the maximum method dendrogram, which suffers from the so-called chaining effect, i.e., the phenomenon that new branches keep being added to a cluster already formed.

In Figure 4.3 both dendrograms are given. In these dendrograms the level at which emotions are connected is an indication of the strength of their relation: The higher in the tree the connection, the more of ten emotions have been confused with one another and the more confusions with particular other emotions they have in common. The structure of the minimum method dendrogram may be described as follows: The positive (or non-negative) emotions of joy, interest, and surprise are grouped together in one cluster; the negative emotions are divided over two clusters, one comprehending the more active ones, namely, disgust, contempt, and anger, and one comprehending the more passive ones, namely, sadness, shame, and, less clearly, fear. Neutral constitutes a cluster by itself. Basically, the same clusters are found in the maximum method dendrogram; only the emotions of interest and shame have changed places.

\subsection{DISCUSSION}

\subsubsection{Classification}

An examination of the order in which variables were inserted in a number of step-wise analysis runs and inspection of the standardized discriminant
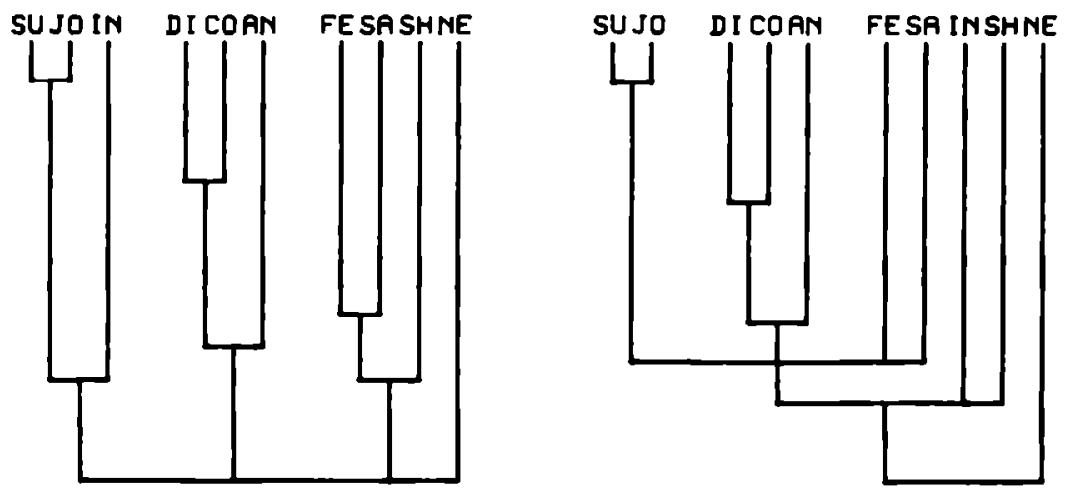

Figure 4.3 Results of a hierarchical cluster analysis based on the classification matrix of a discriminant analysıs with twelve perceptual parameters (mınımum method at the left, maximum method at the right) 
function weights in the twelve variable solution suggested that the best set of discriminating variables includes loudness, laryngeal laxness, pitch level, pitch range, laryngeal tension, and harshness. With the perceptual scores on just the first four of these vocal parameters and three discriminating functions accounting for $92 \%$ of the variance, a percentage correct classification of $58 \%$ was achieved, a percentage which hardly increases if more varables are added: With the full set of twelve discriminating variables and three discriminant functions accounting for $81 \%$ of the variance, $62 \%$ of the material was classified correctly.

It is interesting to compare these percentages with the average performance of human subjects in recognition experiments (of course, it would even be more interesting to compare the number of correct identifications obtained by means of the discriminant analysis with the recognition rate of human subjects judging the same stimuli. This comparison however, will not be presented until Chapter 10 ). In Table 6.1 a survey of twenty-four recognition studies is given. The average accuracy across all these studies was $51 \%$ after correction for chance, a correction which is, of course, necessary if data from studies using different numbers of response categories are to be combined. Thus, it seems that a relatively simple linear procedure like discriminant analysis does not need much more than the perceptual scores on some four very common parameter scales to rival humans in discriminating performance. This applies to a situation in which a single overall score was assigned to each utterance. It could be hypothesized that even better discrimination would be brought about if we were able to deal with variations in loudness, pitch, and tension within an utterance.

\subsubsection{Relative importance of perceptual parameters}

The fact that variables like tremulousness, lip spreading, precision of articulation and whisper hardly contributed to the separation of the emotions they were the last variables to be inserted in the step-wise runs and had very low weights on the functions in the twelve variable solution - does not necessarily mean that they are unimportant for a characterization of vocal expressions of emotion. An explanation of the failure of these variables to contribute to the discrimination of the emotions in our analysis could be that the variables with low contributions to the overall correct classification are of a nature that is fundamentally different from features like loudness, laryngeal tension, pitch level, and pitch range. It is possible that the latter features are used to assign emotional expressions to a not too large number of general classes and that features like tremulousness and lip spreading are used to discriminate emotions within particular classes. In cases like these, a data processing technique which leans entirely on linear combinations of variables in the total space is very unlikely to bring to light the contributions of variables that behave differently in different subspaces. From this we may conclude that it may be ill-advised to use multiple discriminant analysis if 
some of the discriminating variables only apply to a subset of the groups. As a corollary to this conclusion it is clear that one must be very cautious in drawing general conclusions as to the importance of perceptual and acoustical features for the discrimination of emotions; they should at least be viewed against the background of which emotions were used in a particular study.

An indication that one has a situation at hand in which a number of measurement variables apply to only a subset of the groups might be derived from an inspection of the correlation matrix presented in Table 3.3. The least effective variables tremulousness, lip spreading, precision of articulation, and whisper are virtually uncorrelated with any other variable, whereas the effective variables loudness, laxness, pitch level, pitch range, and tension show relatively high mutual correlations. This might be indicative of the fact that we are dealing with two kinds of variables, one that applies to all groups and another, the uncorrelated ones, that applies to some groups. The same impression arose from the a posteriori contrast tests reported on in Chapter 3. According to this line of reasoning it is the fact that the latter variables vary only within some groups but not in others that explains the low correlations for these variables.

These findings fit very well into the hierarchy model proposed by Frijda (1970). Frijda states that "it is possible to conceive of an $n$-dimensional system of emotional space with further differentation in various regions which are specific for and relevant to each region" (p. 247). Frijda speculates that this hierarchical ordering may reflect the process of recognizing an emotional expression, "placing it in a system of gross distinctions and subsequently making finer discriminations" (ibidem).

\subsubsection{Dimensions}

The three functions extracted from the twelve variable solution mainly appeared to consist of different linear combinations of loudness, laryngeal tension, laryngeal laxness, and harshness, complemented with positive contributions of pitch range and pitch level. From this we inferred that all of them represent different aspects of a more general activity dimension. As far as pitch level and pitch range go, this interpretation is in line with previously reported characteristics of the activity dimension (Davitz, 1964c; Huttar, 1968; Scherer \& Oshinsky, 1977). As for loudness, in all relevant studies to date high positive correlations between this parameter and activity ratings have been found (Davitz, 1964c; Huttar, 1968; Scherer, Koivumaki, \& Rosenthal, 1972). At least with respect to the first two dimensions emerging in the present study, these findings also agree with ours. On the other hand, our study did not reveal the consistently reported strong, positive relationship between activity and tempo (Davitz, 1964c; Huttar, 1968; Scherer et al., 1972; Scherer \& Oshinsky, 1977).

As for the first dimension, a justification for an interpretation in terms of level of activity was found in the locations of the emotions along it, going 
from shame, neutral, and sadness at the one end to surprise and anger at the other. Further justification may be found in the outcomes of previous research into the dimensionality of vocal expressions of emotion (Davitz, 1964c; Green \& Cliff, 1975).

Davitz (1964c) had twenty subjects rate vocal expressions of fourteen different emotions on nine scales of the semantic differential, three for each of the three dimensions found by Osgood, Suci, and Tannenbaum (1957). If we look at the five emotions that the present study and Davitz' study have in common, it appears that the relative positions of the emotions on the activity dimension that Davitz found correspond fairly well with the rank order of the emotions on our first dimension. In both studies anger, joy, surprise, fear, and disgust are situated on the positive half of the dimension.

Green and Cliff (1975) had one speaker express the letters of the alphabet with eleven emotions. Seventeen subjects rated the stimuli on seven bipolar scales representing four factors, and indicated the similarity between pairs of stimuli. The similarity judgments, averaged over judges, were subjected to multidimensional scaling, and the ratings on the bipolar scales were factor analyzed. A canonical correlation analysis suggested that both operations yielded the same two dimensions, an evaluation and an excitement or activity dimension. With regard to the latter the locations of the emotions that the present study and Green and Cliff's study have in common agree fairly well with each other. In both studies, anger, joy, surprise and, to a lesser extent, fear are situated towars the active end of the dimension (these results were also obtained by Davitz) and sadness towards the passive end of the dimension. The two studies differ with regard to relative positions of neutral on the one hand, and disgust and contempt on the other, neutral being passive in our study and central in Green and Cliff's study, disgust and contempt being central in our study and passive in Green and Cliff's.

The second and third dimensions emerging from our study we found more difficult to interpret than the first. They do, in any case, not resemble an evaluative dimension.

The fact that our discriminant analysis did not yield an evaluative dimension does not come as a surprise to us. Osgood, Suci, and Tannenbaum (1957) have shown that the general evaluative dimension which comes out of almost any semantic differential experiment as the factor that explains most of the variance can be divided into a number of subdimensfons like moral, aesthetical, social, and emotional evaluation when the right bipolar scales are used with the right set of objects to be rated. In our experiment almost all variables have some relation to activity, and therefore it is only natural that a number of different aspects of activity should emerge.

In fact, it has proven to be rather difficult to detect vocal features that are utilized for the inference of evaluative meaning. Davitz (1964c), for example, who studied the relationship between ratings of timbre, pitch level, loudness, and tempo on the one hand and ratings on scales representing among other 
things the evaluation dimension on the other, did not find any significant correlations. Scherer and Oshinsky (1977) did succeed in finding acoustic parameters that contributed significantly to the attribution of evaluative meaning, namely, fast tempo, few harmonics, large pitch range, sharp envelope, low pitch, pitch contour down, and small amplitude range (listed in decreasing order of predictive strength), but then the stimuli consisted of synthesized tone sequences for which it is impossible to determine whether they were responded to as speech or as music. This makes it also impossible to assess whether the evaluative ratings were related to the attribution of emotional meaning or to the more aesthetic formal qualities of the stimuli. In the latter case the outcomes would not be directly relevant to the topic of the present chapter.

Not only has it proven hard to pinpoint the vocal characteristics of the evaluation dimension, we even wonder if the previous labeling of particular dimensions in terms of evaluative meaning has always been right. It does not seem legitimate, for example, to interpret a dimension that combines joy, anger, and contempt at one extreme, as was the case in the Green and Cliff (1975) study, as an evaluative one. A comparable remark is in order for Dawes and Kramer's (1966) study, where an interpetation as evaluative of the sole dimension extracted would force us to accept sadness as a pleasant emotion. The pressure to try and find an evaluative dimension is perhaps a result of a misinterpretation of the results from Osgood et al. (1957) who consistently found such a dimension but using rating scales that were bound to lead to such a result.

We would finally like to comment on the outcomes of the hierarchical cluster analysis carried out on the classification matrix resulting from the discriminant analysis with the full set of variables. From this cluster analysis two dendrograms resulted, one of which - unfortunately the one which corresponded less well with the distances in the original data matrix - yielded a structure which corresponds very well with what one would intuitively expect, namely one cluster that consists of just neutral, and three clusters comprising the three positive, or at least non-negative emotions of interest joy, and surprise, the three passive negative emotions of shame, sadness, and, somewhat less clearly, fear, and the three active negative emotions of disgust, contempt, and anger, respectively. Thus, by using a technique that yields results in the form of groups of emotions rather than in terms of the relative positions of the emotions along dimensions, it does appear possible to obtain an intuitively plausible structure of the emotions studied, encompassing a positive/negative distinction. This could be taken as an indication that the relationships among the emotions within the groups are more stable than the relationships between the groups of emotions.

Just as we had remarked above in connection with the number of correct classifications, it would, of course, also be interesting to compare the way in which the emotional utterances were classified in the discriminant analysis 
with the classificatory behaviour of human subjects. It would, in other words, be interesting to assess to what extent the relationships among the emotions as they emerged in the present chapter from a consideration of the three dimensional space depicted in Figures 4.1 and 4.2 and from an examination of the dendrograms given in Figure 4.3 correspond with the relationships among the emotions as they appear from a recognition experiment in which the subjects are confronted with basically the same task, namely that of assigning each utterance to one of the ten categories examined. This topic will also be dealt with in Chapter 10.

\section{NOTES}

1. It would be more correct to use "fundamental frequency" or " $F_{0}$ " rather than "pitch" for the acoustic parameter. However, pitch, the term for the perceptual representation of $F_{0}$, has been used throughout the chapter since it makes for easier reading.

2 The description of the principles of discriminant analysis given in this chapter is largely based on the information provided in Chapter 23 of the SPSS manual (Klecka, 1975). 


\section{Acoustic correlates of perceptual} parameters

\subsection{INTRODUCTION}

In Chapter 3 a description was given of the ten emotional categories under study in terms of global perceptual ratings on twelve vocal parameter scales. Not much is known about the physical cues on which such ratings are based. This lack of knowledge is one of the great obstacles (for other complicating factors, see Section 3.4.4) to the integration of findings from different studies on the vocal characteristics of emotion, and we therefore think that the search for objective, physical measures to describe global characteristics of running speech deserves more attention than it has hitherto received. If it is possible to find acoustic measures, or combinations of them, which correlate highly with auditory ratings of particular relevant vocal speech characteristics and do so consistently for ratings obtained from different (groups of) raters with respect to different speech materials, we consider these measures to be meaningful and appropriate for use as standardized tools for describing emotional expressions.

In the present chapter the results will be presented of an attempt to find the best possible acoustic correlate, selected each time from a limited number of plausible candidates, for perceived tempo, pitch level, pitch range, harshness, loudness, laryngeal tension ("tension" from now on), and laryngeal laxness ("laxness" from now on). The decision to look for correlates of these specific perceptual parameters is partly motivated by the outcomes of the discriminant analyses described in Chapter 4: Except for tempo, these parameters appeared to constitute the best set of variables for separating the ten emotional categories under study. Moreover, this set of variables comprises those which in emotion research to date have been considered most often.

In those cases in which we have succeeded in finding a reasonably satisfactory correlate for one of the parameters mentioned, we will also give the numerical values for the different emotions. These numerical data will not be commented on, nor will they be compared to acoustic data reported in other investigations since that would amount to repeating to a great extent what has been done in Section 3.4.4. The data will hopefully be of use, however, to other researchers who wish to compare their acoustic findings. 
The structure of this chapter is as follows: In Section 5.2.1 a short description will be given of the nature of the perceptual ratings serving as criteria in the present chapter. In Sections 5.2.2 to 5.2.4 we will present the acoustic measures that we have considered as potential correlates of perceived tempo, pitch level, pitch range, harshness, and loudness, tension, and laxness, respectively. Then, in Sections 5.3.1 to 5.3.5, the results of the correlations between these acoustic measures and their perceptual counterparts will be given. On the basis of these results, for each perceptual parameter one acoustic correlate will be selected, except for loudness, tension, and laxness, which are represented by a composite acoustic measure. In Section 5.3.6 the results will be presented of a multiple regression analysis in which all of the perceptual parameters are correlated with the complete set of selected acoustic measures, and in Section 5.3.7 the outcome will be given of a discriminant analysis with the set of eventually retained acoustic measures as discriminating variables. Finally, in Section 5.4, the findings will be discussed.

\section{S.2 METHOD}

\subsection{Perceptual ratings}

The perceptual ratings which in the present study we tried to represent by means of acoustic measurements were the averaged values over six raters for each of 160 emotional utterances on tempo, pitch level, pitch range, harshness, loudness, tension, and laxness. The 160 utterances consisted of 8 speakers ( 4 males and 4 females) $\times 2$ phrases (twee maanden zwanger and $z 0^{\prime} n$ grote Amerikaanse auto) $\times 10$ emotions (neutral [NE], disgust [DI], surprise [SU], shame [SH], interest [IN], joy [JO], fear [FE], contempt [CO], sadness [SA], and anger [AN]). (For a detailed description of the rating procedure, see Chapter 3; for a detailed description of the speech material, see Chapter 2.)

\subsubsection{Measures of tempo}

In the rating sessions, the raters had been asked to score the parameter tempo in terms of rate of articulation, i.e., the amount of speech produced per time unit, disregarding the pauses, if any. As a physical measure to explain the ratings we took the number of syllables per second, a measure which - in contrast to total duration - is independent of the differing lengths of the two phrases measured (the realizations of twee maanden zwanger were on the average shorter than those of $z 0^{\prime} n$ grote Amerikaanse auto).

In order to check whether the raters had in fact disregarded the pauses in their judgment, the measure was computed twice, once for the integral utterances including the pauses and once after the pauses had been subtracted from the utterance durations. For the determination of the pauses use 
was made of the circumstance that during the rating sessions the raters, along with noting down their scalar judgments, had been asked to indicate for each utterance whether it contained any pauses or not, and, if they thought it did, to mark the place. For the present purpose only those acoustic silences were taken to be pauses which had been marked as such by at least five of the six raters. There were fifteen pauses that met this criterion, ranging in duration from 90 to $1,888 \mathrm{~ms}$, with a mean of $936 \mathrm{~ms}^{1}$

The measurements were carried out with the speech editing system MOSES (Buiting, 1981). This system enables one to accurately determine the boundaries of one or more segments of a speech signal on the basis of auditory and visual information. The duration of the isolated segments is displayed on a terminal screen. The two syllable-per-second measures were obtained by dividing the total duration values, once including and once excluding the pauses, by the number of syllables derived from the written version of the two phrases, i.e., five in the case of twee maanden zwanger and ten in the case of zo'n grote Amerikaanse auto.

\subsubsection{The measurement of fundamental frequency}

Perceived pitch level, perceived pitch range, and perceived harshness are commonly thought to be related to fundamental frequency $\left(F_{0}\right)$, i.e., to (variations in) the rate of vibration of the vocal folds. In the present study fundamental frequency data were derived from computer processing of the output of an analog pitch period detector described in Van Rossum and Boves (1978). This device delivers fundamental period durations with a resolution of $1 \mu \mathrm{s}$ in those cases in which a speech sound has been classified as voiced.

The sequence of fundamental period durations has been processed in a number of ways in order to produce different $F_{0}$ measures. For most of the measures an error correcting algorithm forms part of the processing, which means that obvious outliers are removed. The use of error correction is necessitated by the finding that no pitch extractor performs error-free for wide ranges of voices and speech material. The error-corrected $F_{0}$ information is additionally transformed into a representation that consists of one $F_{0}$ value per $10 \mathrm{~ms}$ time interval.

\subsubsection{Measures of central tendency of $F_{0}$}

It is known that the perceived overall pitch of a complex stationary sound depends primarily on the frequency of the fundamental (Plomp, 1976). The perceived pitch level of sounds in which the fundamental frequency varies has been investigated less thoroughly but it seems reasonable to expect that it will be related to some measure of the central tendency of the $F_{0}$. We have experimented with all three common measures of central tendency, viz. the arithmetic mean (henceforward simply referred to as the mean), the median, and the mode. The reason for considering the modal value along with the 
mean is that it might be a better indicator of the global impression of pitch level since it is the $F_{0}$ that is present most often. The median value, on the other hand, is much less affected by the presence of outliers, a property which is particularly important when the distribution consists of a relatively small number of samples as is the case in the present study.

\subsubsection{Measures of $F_{0}$ variation}

The most promising acoustic correlate of perceived pitch range is a measure of the variation in the $F_{0}$ distribution. As is the case with measures of central tendency, various measures of variation can be defined. In the choice of a measure several criteria must be taken into account, such as its statistical properties, its computational complexity, and, of course, whatever a priori knowledge is available about the human pitch perception mechanism.

To begin with the last criterion, it is well known that the human perception of pitch intervals obeys a logarithmic law, i.e., to the human auditory system a pitch interval between $100 \mathrm{~Hz}$ and $200 \mathrm{~Hz}$ is equal to the interval between $200 \mathrm{~Hz}$ and $400 \mathrm{~Hz}$, each being an octave. One way of taking into account the logarithmic properties of the auditory system is to use a measure of relative range such as the variation coefficient, defined as the quotient of the standard deviation and the mean of the $F_{0}$ distribution. This is the first acoustic measure that we considered.

Another way in which the properties of the auditory system can be accounted for is by transforming linearly scaled $\mathrm{F}_{0}$ data expressed in $\mathrm{Hz}$ into semitone values. Choosing an $\mathrm{F}_{0}$ of $50 \mathrm{~Hz}$ as the base level of 0 semitones, this transformation was effected by means of the relation $12 .{ }^{2} \log \left(\mathrm{F}_{0} / 50\right.$ ) (in semitones). After this log-transform the standard deviation of the distribution was taken as a second measure of variation. Finally, the difference between the 90th and the 10th percentile points was also considered.

Thus, three measures of $F_{0}$ variation were computed as possible correlates of perceived pitch range.

\subsubsection{Measures of $F_{0}$ perlurbations}

There are quite a few studies which suggest that the perceptual impression of roughness or harshness ${ }^{2}$ is related to cycle-to-cycle fluctuations in the duration of $F_{0}$ periods (for an overview of the literature, see Askenfelt \& Hammarberg (1981) and Laver (1980, p. $127 \mathrm{ff}$ ). However, even in speech that does not sound rough or harsh slight variations in $F_{0}$ are constantly present.

This last observation can be reflected in one measure of $F_{0}$ perturbation, defined as the proportion of $F_{0}$ periods that differ more than a given percentage from their neighbours. The threshold must be set in such a way that $F_{0}$ movements associated with intonation are prevented from contributing to the measure. $A$ way to avoid problems with $F_{0}$ variations induced by intonation is to use another $F_{0}$ perturbation measure, defined as the proportion of times that the slope of the $F_{0}$ curve as function of time changes its sign. A third way to approach the problem is to use statistical properties of the 
distribution of differences between adjacent $F_{0}$ samples as a measure of $F_{0}$ perturbation. The differences cannot be taken in an absolute way as it has been found that the same deviation will sound less harsh as it is superimposed on a fundamental of higher frequency (Wendahl, 1963).

Askenfelt and Hammarberg (1981) have compared measures of all three types described with respect to their ability to account for the perceived voice quality of forty-one patients both before and after voice therapy. They conclude that a measure based on the variation of the $F_{0}$ difference distributions is the most promising one, especially in situations in which the $F_{0}$ perturbation measure is to be derived from automatic processing of tape recordings. Measures of the first two types mentioned appear to be extremely sensitive to occasional errors of the pitch extractor used.

We have implemented two $F_{0}$ perturbation measures based on the relative differences between adjacent $F_{0}$ samples. Both measures are computed from the original period duration data before their conversion to $F_{0}$ samples at a $10 \mathrm{~ms}$ rate. The difference between the two measures lies in the presence or absence of an error correction algorithm. The relative $F_{0}$ difference $d F_{n}$ is defined by:

$$
\mathrm{dF}_{n}=\frac{\mathrm{F}_{n}-\mathrm{F}_{n-1}}{.5\left(\mathrm{~F}_{n}+\mathrm{F}_{n-1}\right)}
$$

where $\mathrm{F}_{n}, n=1,2, \ldots N$, are $\mathrm{F}_{0}$ values as read by the computer. $\mathrm{F}_{n}$ does not exist if the sample $F_{n}$ is the first sample of a voiced interval since in that case the preceding sample $F_{n-1}$ cannot be defined meaningfully. The $F_{0}$ perturbation measures are defined by:

$$
\mathrm{F}_{0} \text { perturbation }=\frac{1}{N} \stackrel{N}{N=1}_{n}^{N}\left|\mathrm{dF}_{n}\right|
$$

Since the expected value of the $\mathrm{dF}_{n}$ is very close to zero, the use of the mean of $\left|\mathrm{dF}_{n}\right|$ makes the measure very much like the standard deviation measure coming out as the best in the comparative study of Askenfelt and Hammarberg (1981).

\subsubsection{Measures of spectral slope}

If a one-parameter curve is fitted through the spectra of voiced speech sounds of normal loudness and tension, it is generally found to fall off at a rate of approximately $6 \mathrm{~dB}$ per octave. It is very likely, however, that the spectral slope is affected by variations in loudness and tension. That is why we considered spectral slope measures as possible correlates of perceived loudness, tension, and laxness. ${ }^{3}$ 
The spectral slopes were measured by means of critical bandwidth power spectra. Critical band spectra approximate the frequency resolving power of the human auditory system (Plomp, 1976) and are easy to obtain and process (Pols, 1977; van Rossum, 1981). Our analyser comprises nineteen bandpass filters, namely sixteen third-octave filters at center frequencies from $400 \mathrm{~Hz}$ up to and including $12,500 \mathrm{~Hz}$ and three filters with a fixed $90 \mathrm{~Hz}$ bandwidth and center frequencies of 122,215 , and $307 \mathrm{~Hz}$. All filters are provided with detectors with logarithmic amplitude response and time constants in the order of $10 \mathrm{~ms}$. In addition, a true Root Mean Square detector which measures the sound power in the frequency band from $80 \mathrm{~Hz}$ up to $14,000 \mathrm{~Hz}$ is provided. The twenty detector outputs are sampled at a rate of 100 sa mples per second and, combined with the fundamental frequency values and the voiced/unvoiced outputs of the pitch detector described above, fed into a minicomputer.

Each frame of twenty-two numbers in the computer memory describes properties of the speech signal during a $10 \mathrm{~ms}$ time interval. The detailed output of these measurements depends heavily on the specific phonetic identity of the phonemes to which they relate and is of no great interest if one is looking for acoustic concomitants of global auditory judgments. Therefore, some kind of time averaging is needed. Since we are mainly interested in spectral measures associated with laryngeal behaviour we decided to compute time averages over the $10 \mathrm{~ms}$ intervals pertaining to voiced segments only, separately for each of the 160 utterances. The resulting long-term average spectra (henceforward abbreviated as LTAS) were subsequently normalized by expressing the power level in all filter channels relative to the overall signal power scaled to an arbitrarily chosen fixed level of $100 \mathrm{~dB}$. This effectively removes all between-differences in total signal power.

Unlike the acoustic measures considered as correlates of tempo, pitch level, pitch range, and harshness, which result in a single number for each utterance, the LTAS comprise nineteen significant numbers, thus constituting a measure which calls for simplification. Fortunately, from the work on critical band analysis applied to speech signals it appears that the levels in adjacent filters tend to be fairly strongly correlated (Pols, 1977) so that a certain amount of data reduction should be possible without the loss of relevant information.

The data reduction on the LTAS can be achieved in two fundamentally different ways. The first way is to use a formal multivariate data processing technique that maps the original nineteen dimensional data space onto a new space of lower dimensionality while preserving the greatest possible amount of variance. The alternative approach does not attempt to use "blind" statistical techniques to save a maximum amount of variance or information in a statistical sense. Rather it considers the possibility that not all variance is equally important for the goal to be reached and consequently tries to extract that information that is expected to be relevant for reasons based on a priori 
knowledge about the phenomena under study. Such an approach is exemplified by the spectral slope measures defined by Hammarberg et al. (1980).

Boves and Cranen (1982) compare the two approaches. They conclude that the differences are too small to warrant their concurrent application. Since the spectral slope measures as defined by Hammarberg et al. are easier to interpret, this approach is preferred in the present study. The slope measures are defined in Figure 5.1. The maximum level in the bands with center frequencies 400,500 , and $630 \mathrm{~Hz}$ is taken as the reference with respect to which all remaining levels are measured. This band usually contains the first formant, the level of which contributes most to the overall power of the speech wave.

In the LTAS measure derived from Hammarberg et al. the first slope factor ${ }^{4}$ is determined by the level in the filter which contains the $F_{0}$ mode. The second slope factor is defined by the level in the filter with a center frequency of $1,600 \mathrm{~Hz}$, and the third by the level at $5,000 \mathrm{~Hz}$ (a point at which the level of the harmonics of the glottal pulses is usually well below the system noise). The fourth, and last, slope factor depends on the maximum level in the frequency region above $5,000 \mathrm{~Hz}$. Since our data are confined to voiced segments, this level differs only very slightly from the level at $5,000 \mathrm{~Hz}$. Therefore, we have limited our data processing to the first three slope factors.

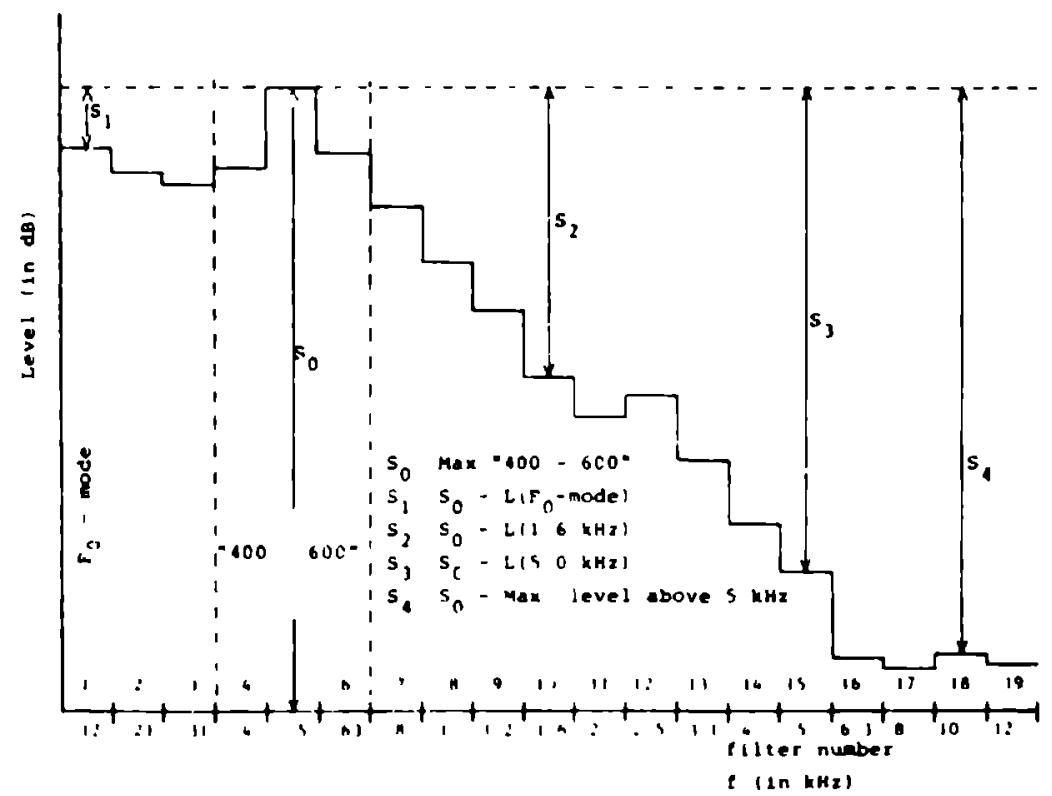

Figure 5. I Definition of LTAS slope measures 


\subsection{RESULTS}

\subsubsection{Ratings of tempo related to syllable duration}

Two measures were considered as acoustic correlates of perceived tempo:(1) the number of syllables per second with pauses and (2) the number of syllables per second without pauses. In Table 5.1 the product-moment correlations between the physical and the perceptual speech rate measures are presented.

From Table 5.1 it appears that the speech rate measure with and the speech rate measure without pauses correlate so highly with each other that one wonders whether one really has two different measures at hand. The high correlation is probably due to the fact that in our speech material only 15 out of the 160 utterances contained a pause, so that for the great majority of the phrases the duration values for the two measures were the same.

It may further be seen that of the two duration measures the one without pauses correlates more highly with tempo than the measure with pauses. This outcome suggests that the raters have indeed concentrated on rate of articulation and have paid little attention to the pauses. In fact, the correlation between the number of syllables per second without pauses and its perceptual analog may be seen to be quite high. It was therefore used as one of the acoustic predictors in the multiple regression analysis described in Section 5.3.6. (For the mean values for the different emotions, see Appendix A.)

\subsubsection{Ratings of pitch level explained by measures of central tendency of $F_{0}$}

Three measures of central tendency of $F_{0}$ were considered as possible correlates of perceived pitch level: (1) the mode, (2) the median, and (3) the mean. Since the raters had been asked to keep in mind the sex of the speaker while judging the height of the pitch - which really amounts to asking them to use a normalized rating scale - the acoustic data were converted to $z$-scores separately for the utterances within etther sex group, before being combined and correlated with the perceptual ratings. The resulting product-moment correlation matrix is given in Table 5.2.

Table $S I$ Intercorrelations between two rate measures and perceived tempo $(N=160)$

\begin{tabular}{lll}
\hline & 1 & 2 \\
\hline 2 & $87^{*}$ & \\
3 & $77^{*}$ & $85^{*}$ \\
\hline
\end{tabular}

$1=$ syllables per second with pauses $2=$ syllables per second without pauses $3=$ perceived tempo

- $p<05$ (one-talled test) 
Table 52 Intercorrelations between three measures of central tendency of $F_{0}$ and perceived pitch level $(N=160)$

\begin{tabular}{|c|c|c|c|c|}
\hline & 1 & 2 & 3 & \\
\hline 2 & $92^{*}$ & & & $1=F_{0}$ mode \\
\hline 3 & $87 *$ & $97 *$ & & $2=F_{0}$ median \\
\hline 4 & $71^{*}$ & $80^{\circ}$ & 82 & $3=F_{0}$ mean \\
\hline
\end{tabular}

From Table 52 it appears that the $F_{0}$ mode is a less effective predictor of the pitch level ratings than the $F_{0}$ median and the $F_{0}$ mean. The latter two measures do about equally well and correlate extremely highly with each other Apparently, during the $F_{0}$ processing, outliers, which tend to make the $F_{0}$ mean unstable, have been effectively removed Nevertheless, since for general use we think that the $F_{0}$ median is to be preferred above the mean, in the subsequent multiple regression analysis the $F_{0}$ median was employed. (For the mean $F_{0}$ median values for the different emotions, see Appendix A.)

Since we were interested to know whether the raters really employed two different scales in their judgments of pitch level, we also computed the correlations between the non-normalized $F_{0}$ median data and the pitch level ratings separately for the utterances produced by the male speakers, the female speakers, and the combined group of 160 utterances. The coefficients were 86,80 , and .75 , respectively, which suggests that the ratıngs of the utterances do indeed depend to some extent upon the sex of the speaker. From the fact that the correlation between the normalized $F_{0}$ median data and the perceptual ratings for the combined group of 160 utterances was 80 we may conclude that part of the difference between the pitch level scales for the male and the female speakers is confined to a linear transformation.

\subsubsection{Ratings of pitch range explained by measures of $F_{0}$ variation}

Three acoustic measures were considered as possible correlates of perceived pitch range: (1) the variation coefficient, (2) the standard deviation of the log-converted (semitone) $F_{0}$ distribution, and (3) the difference between the 90 th and the 10th percentile points in this distribution The product-moment correlations between these three measures and the pitch range ratings are given in Table 5.3

It appears from Table 53 that the correlations between the three acoustic measures are high Since the vanation coefficient does relatively best in predicting the perceptual ratıngs and does not involve the extra computational complexity of the log-transform we decided to choose it as the measure to be employed in the subsequent multiple regression analysis. (For the mean variation coefficient values for the different emotions, see Appendix A) 
Table 5.3 Intercorrelations between three measures of $\mathrm{F}_{\mathrm{o}}$ variation and perceived pitch level $(N$ $=160$ )

\begin{tabular}{llll}
\hline & 1 & 2 & 3 \\
\hline 2 & $.88^{*}$ & & \\
3 & $.95^{*}$ & $.90^{*}$ & \\
4 & $.68^{*}$ & $64^{*}$ & $.60^{*}$ \\
\hline
\end{tabular}

$-p<.05$ (one-tailed test)

$1=F_{0}$ variation coefficient

$2=$ standard deviation of the logconverted $F_{o}$ distribution

$3=$ difference between the 90 th and 10th percentile of the log-converted $F_{0}$ distribution $4=$ perceived pitch range

\subsubsection{Ratings of harshness explained by measures of $F_{0}$ perturbations}

Two acoustic measures were considered as possible correlates of perceived harshness: (1) an $F_{0}$ perturbation measure computed from error-corrected $F_{0}$ data and (2) one which was based on uncorrected $F_{0}$ data. In Table 5.4 the correlations between the two measures and the harshness ratings are given.

The data in Table 5.4 show that neither perturbation measure is a very good predictor of perceived harshness. The correlation between the corrected perturbation coefficient and the harshness ratings is not even significant and so low as to be devoid of any meaning. The correlation between the uncorrected perturbation coefficient and perceived harshness is not very impressive either: The acoustic measure accounts for only $17 \%$ of the variance in the perceptual ratings. For lack of something better we nevertheless decided to keep it as an acoustic correlate of perceived harshness.

5.3.5 Ratings of loudness, tension, and laxness explained by measures of spectral slope

The LTAS measures differ from the tempo and $F_{0}$ measures in that one may not expect to find meaningful results from simple product-moment correlations between the acoustic and the perceptual data. Rather one must have recourse to multivariate statistical techniques. We have chosen an approach based on a step-wise multiple regression analysis.

Because in Chapter 3 it appeared that the ratings for loudness, tension, and laxness were correlated (see Table 3.3), we thought it sensible to present the results for these three parameters in combination. We have tried to obtain a

Table 5.4 Intercorrelations between two $\mathrm{F}_{0}$ perturbation measures and perceived harshness $(N$ $=160$ )

\begin{tabular}{lll} 
& 1 & 2 \\
\hline 2 & $.56^{*}$ & \\
3 & $.42^{*}$ & .14 \\
\hline
\end{tabular}

$$
\begin{aligned}
& 1=\text { uncorrected } F_{o} \text { perturbation } \\
& 2=\text { corrected } F_{o} \text { perturbation } \\
& 3=\text { perceived harshness }
\end{aligned}
$$

$* p<.05$ (one-talled test) 
maximally transparent and compact presentation of the multıple regression outputs in Table 55 . The order in which the slope measures were inserted into the regression equation can be inferred from the cumulative contributions to the total amount of variance explained. The threshold for insertion was set at $5 \%$.

From Table 5.5 it may be seen that tension and laxness show the expected complementary picture: For both parameters the first two slope measures are most important, but the order in which they enter the equation and their relative contributions to the total a mount of variance explained are reversed. Comparing the results for tension and loudness, we see that they are partly different and for another part quite similar. The difference lies in the relative importance of the slope measures For the perception of loudness the spectral slope in the frequencies below the first formant is most important, followed by the slope in the frequency region around $5,000 \mathrm{~Hz}$; for the perception of tension the slope in the frequencies between the first formant and $1,600 \mathrm{~Hz}$ is most important, immediately followed by the spectral slope below the first formant The similanty lies in the signs of the regression coefficients, which suggest that higher degrees of both loudness and tension are characterized by a higher spectral level (or, in our measures, a less steep spectral slope) in the upper frequency bands.

\subsubsection{A multiple regression approach}

So far we have considered each perceptual parameter individually. This approach is motivated by the claimed analytic character of the perceptual ratıngs The fact, however, that we found some of the rating scales to be correlated (see Table 33 ) suggests the possibility that a number of different acoustic parameters might interact to determine the perceptual scores This possibility was examined by carrying out a multiple regression analysis with the eventually retained acoustic measures, 1.e., number of syllables per second without pauses, $F_{0}$ median, $F_{0}$ variation coefficient, uncorrected $F_{0}$ perturbation, and three spectral slope measures, as predictors and the seven ratıng scales as criteria The threshold for insertion was set at $5 \%$. The results are given in Table 5.6.

Table S 5 Results of a multıple regression analysıs with perceptual ratıngs as criterion and spectral slope measures as predictors The numbers denote cumulative percentages of variance explained by the slope measures The signs are borrowed from the regression coefficients

\begin{tabular}{llll}
\hline & Loudness & Tension & Laxness \\
\hline Slope 1 & 32 & 39 & $20(-)$ \\
Slope 2 & & $21(-)$ & 34 \\
Slope 3 & $51(-)$ & $46(-)$ & 59 \\
Multiple $R$ & 72 & 68 & 59 \\
\hline
\end{tabular}


Table 56 Results of a multıple regression analysis with ratıngs on seven perceptual parameters as criterion and seven acoustic measures as predictors The signs are borrowed from the regression coefficients

\begin{tabular}{llll}
\hline Criterion & Predictor(s) & Multiple $R(r)$ & $\begin{array}{l}\text { Cumulative \% } \\
\text { variance explained }\end{array}$ \\
\hline Tempo & Syllables per second & 85 & 73 \\
Pitch level & Fo median $_{0}$ & 80 & 64 \\
Pitch range & Fo variation coefficient & 68 & 47 \\
Harshness & Fo perturbation & 62 & 18 \\
& Slope 3 & & $26(-)$ \\
& Slope 1 & & 32 \\
& Syllables per second & 72 & 38 \\
Loudness & Slope 1 & & 32 \\
& Slope 3 & 76 & $51(-)$ \\
Tension & Fo median & & 28 \\
& Slope 3 & & $43(-)$ \\
& Fo perturbation & & 51 \\
& Slope 1 & 59 & $20(-)$ \\
Laxness & Slope 1 & & 34 \\
& Slope 2 & & \\
\hline
\end{tabular}

For tempo, pitch level, and pitch range the results presented in Table 5.6 are extremely easy to describe, since no acoustic measures beyond the number of syllables per second without pauses (for tempo), $F_{0}$ median (for pitch level), and the $F_{0}$ variation coefficient (for pitch range) appear to make a significant contribution The same holds for the laxness and loudness scales, where no significant contributions are found from parameters other than the slope measures.

For tension, slope 2 is removed from the equation and replaced by the $F_{0}$ median. Slopes $I$ and 3 exchange places, and $F_{0}$ perturbation comes in as a fourth predictor. The regression coefficients of both $F_{0}$ median and $F_{0}$ perturbation are positive, indicating a tendency in the direction of higher tension scores if either $F_{0}$ median or $F_{0}$ perturbation increase. This is what should be expected on physiological grounds A physiological explanation is corroborated by the regression coefficients of the slope measures which are negative for the high frequency slope 3 and positive for the low frequency slope 1 .

For harshness, $F_{0}$ perturbation remains the most important predictor, but it is joined by slope measure 3, slope measure 1 , and the duration measure, yielding a multiple $R$ of .62 compared with a Pearson $r$ of 42 for perturbation as the sole predictor Moreover, the signs of the regression coefficients of the slope measures are equal to those for tension. This finding supports the conjecture that harshness is likely to be one of the results of excessive tension of the larynx muscles (see Laver, 1980, p. 129). 


\subsubsection{Discriminant analysis}

In order to get an impression of the discriminating power of the set of seven acoustic measures used in the multiple regression described above, a discriminant analysis (for an explanation of this technique, see Section 4.2.2) was carried out. With three discriminant functions accounting for $91 \%$ of the variance, 69 of the 160 utterances, i.e., $43 \%$, were assigned to the right emotional category. The percentages correct for the different emotions are given in Table 5.7.

The heaviest contributions to the first function, which accounted for $58 \%$ of the variance, came from $F_{0}$ median and the $F_{0}$ variation coefficient (both positive); the heaviest contributions to the second function, which accounted for an additional $24 \%$ of the variance, came from syllables per second and the first slope measure (both positive) as well as from $F_{0}$ median (negative). The contributions of the other variables were low. The third function extracted will not be considered, since it accounted for only $9 \%$ of the variance.

In Figure 5.2 the positions of the group centroids in the space spanned by the first and second discriminant functions (dimensions) are depicted. It may be seen that the passive emotions neutral and shame are situated at one extreme of the first dimension and the active emotions joy and surprise at the other. The second dimension goes from disgust to interest, and is not easy to interpret.

Just as has been done with respect to the perceptual ratings (see Section 4.3.3), a hierarchical cluster analysis was carried out on the classification matrix yielded by the acoustically based discriminant analysis in order to establish which emotions were most often confused with one another. Both dendrograms, resulting from the application of the minimum method and the maximum method, are presented in Figure 5.3. Since the correlation between the distances in the data matrix and the ultrametric distances was considerably higher for the minimum method than for the maximum method (.70 and .55 , respectively), we will only consider the former. It contains four clusters of emotions, one comprising disgust and contempt, one comprising

Table 5.7 Percentages of correct classification by means of a discriminant analysis based on seven acoustic measures

$\begin{array}{ll}\text { Neutral } & 50 \\ \text { Disgust } & 44 \\ \text { Surprise } & 62 \\ \text { Shame } & 38 \\ \text { Interest } & 69 \\ \text { Joy } & 31 \\ \text { Fear } & 38 \\ \text { Contempt } & 31 \\ \text { Sadness } & 19 \\ \text { Anger } & 50\end{array}$




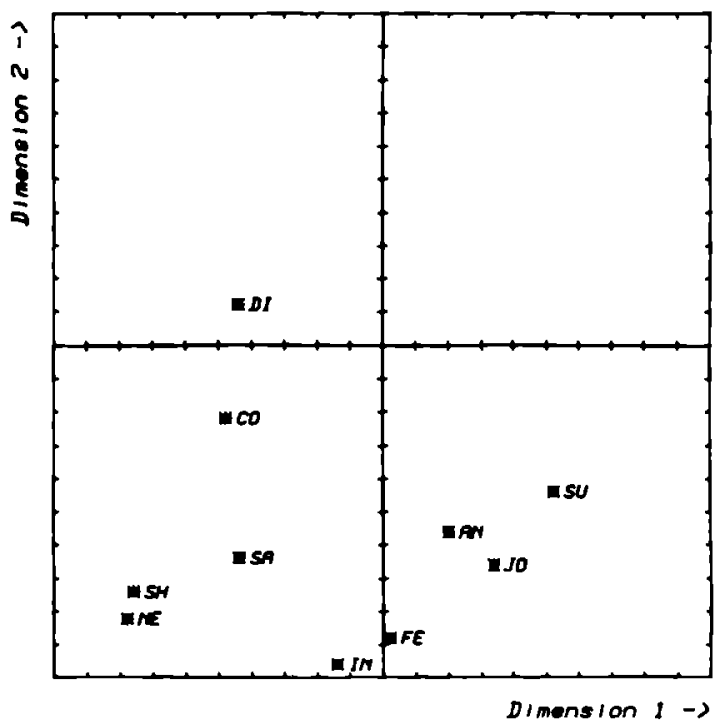

Figure 5.2 Positions of ten emotions along the first and second dimensions resulting from a discriminant analysis based on seven acoustıc measures
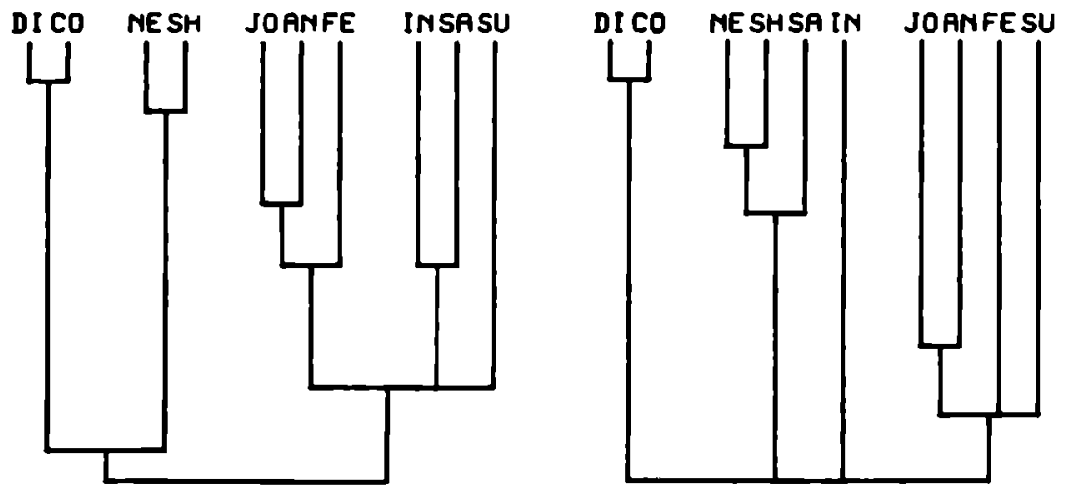

Figure 5.3 Results of a hierarchical cluster analysis based on the classification matrix of a discriminant analysis with seven acoustıc parameters (mınımum method at the left, maximum method at the right)

neutral and shame, one comprising joy, anger, and fear, and one comprising interest, sadness, and surprise.

\subsection{DISCUSSION}

The present study revealed that ratings of tempo, or, more precisely, those of rate of articulation, of single utterances can to a great extent be predicted by the number of syllables per second, calculated after subtraction of the 
pauses. In fact, of all the perceptual parameters that were examined in the present study, tempo was the one for which the search for a physical correlate proved most successful. Of the variance in the perceptual tempo ratings that is not accounted for by the physical measure ( $28 \%)$, some may be due to error on the part of the raters; another part may be related to tempo changes within utterances.

The ratings of pitch level can for the greater part be accounted for by measures of the central tendency of the $F_{0}$ distribution. Although this result was to be expected from knowledge about similar relations for stationary sounds, it is gratifying to find that they hold for highly non-stationary sounds such as our emotional utterances as well. However, here also part $(36 \%)$ of the variance in the perceptual ratings remains unexplained. Again, this may, at least partly, be a result of insecurity on the part of the raters, perhaps due to interference from spectral properties of the speech signal.

Our study suggests the presence of a sex of speaker factor in the rating of pitch level, but its importance and its exact effect are not quite clear. In a rating experiment in which five male and five female speakers were judged by means of a semantic differential which included the scale low pitch - high pitch without further reference to the sex of the speaker, Blom and Van Herpt (1976) found that the scores of untrained raters could not be sensibly scaled by means of Thurstone's Law of Categorical Judgement. This result was also taken as an indication that norms for pitch height are sex dependent.

One final remark is in place on the relation between $F_{0}$ median and perceived pitch level: Spectral properties of the signals expressed in terms of slope measures did not contribute any significant amount of explained variance. This does not prove that spectral effects have not played a part in the pitch level rating - on the contrary, as mentioned above we think it probable that some interference has taken place - but rather that the slope measures are too rough to account for these effects.

A similar remark applies to the relation between measures of $F_{0}$ variation and ratings of pitch range. There the addition of slope measures as predictors did not lead to a higher percentage of explained variance either. It furthermore appeared that the explanatory power of the $F_{0}$ variation measures was not very high: With the best of the three measures considered, not more than half of the variance in the perceptual ratings could be explained.

Again this may be due to the inaccuracy of the perceptual ratings. 't Hart (1981) investigated the precision with which (trained and untrained) subjects were able to judge which one of two $F_{0}$ movements in pairs of synthesized four-syllable number names was greater. Of the untrained subjects $16 \%$ were not able to discriminate between differences of less than four semitones in the case of $F_{0}$ rises. In judging $F_{0}$ falls, the proportion of judges who could not perform the task of discriminating below a four semitone threshold rose to no less than $61 \%$. From these results we might hypothesize that the mediocre 
explanatory power of acoustic $F_{0}$ measures of pitch range is at least partly due to uncertainty on the part of the raters in estimating the pitch range accurately, especially in those utterances in which the range is neither extremely narrow nor extremely wide.

Of the two acoustic measures considered as possible correlates of perceived harshness, the more effective one accounted for no more than $17 \%$ of the variance in the perceptual ratings. This poor result may have to do with the nature of the speech material analyzed in this experiment. After all, all utterances were produced with normal voices. The occasionally perceived harshness, which occurred especially in expressions of disgust, joy, and anger, was usually not very severe and often intermittent. Those studies in which perturbation measures proved to be more successful dealt with the discrimination between normal voices and pathological voices for which irregularity was a characteristic feature (Askenfelt \& Hammarberg, 1981). At this moment we cannot do much more than conclude that extensive research is needed regarding the perception of (the degree of) harshness in normal voices. Preferably that research should employ stretches of speech much longer than 5 seconds so that stable automatic acoustic measures of $F_{0}$ perturbation can be obtained. ${ }^{5}$

The results concerning the spectral slope measures are impossible to explain without reference to a parametric representation of glottal pulse shapes. The most important parameters of this representation are period duration (the inverse of $F_{0}$ ), the amplitude of the pulses, and the slope of the trailing edge (Fant, 1979; Titze, 1980). It can be shown that the effect of $F_{0}$ on slope measures is negligible; thus it cannot be a point in the present discussion (Boves, 1984). (This is not to say that $F_{0}$ is not related to loudness and tension; it certainly is, but this relation does not affect the slope measures per se).

Increasing pulse amplitude without changing the waveform, which, in a first approximation, could be done by increasing the subglottal pressure and/or by causing the vocal folds to make larger vibratory excursions, increases the loudness of the radiated speech signal. However, it must be assumed that a large part of this kind of loudness variations, if they were present in the signals as produced by the speakers, have been removed by level adjustments during the recording and copying processes, and are consequently not present in the LTAS obtained.

In addition to increasing pulse amplitude, tensing the laryngeal muscles may also contribute to the impression of increased loudness, and - of course to the impression of increased tension as well. Pressing the vocal folds together more firmly and increasing the longitudinal tension of the folds will both steepen the trailing edge and decrease the average pulse amplitude. It follows from standard Fourier analysis that the former effect corresponds to an increase in the level of the higher harmonics; in other words, steepening 
the trailing edge of the glottal pulse changes the spectral balance in favour of the upper part of the spectrum. For most common pulse forms, the latter effect will be reflected in the spectral level at the fundamental frequency.

From the fact that both perceived loudness and perceived tension are positively related to the first slope measure, it appears that either a decrease in the relative spectral level of $F_{0}$ (due to a decrease in pulse amplitude) or a relative increase in $F_{1}$ level (due to a steeper slope of the flow pulse at glottal closure) indeed gives rise to an increase in perceived loudness and tension. Moreover, from the negative signs of the regression coefficients for slope measures 2 and 3 it can be inferred that a rise in the spectral level at higher frequencies (showing up in the form of small values of slope measures 2 and 3 ) also corresponds with increasing scores on loudness and tension. Finally, as one would expect, increasing the spectral slope measures towards the higher frequencies, i.e., favouring the low instead of the high frequencies, leads to higher scores for laxness.

Not surprisingly, loudness and tension are very similar as far as the acoustic predictors are concerned but not identical. We will not speculate on the underlying physiological mechanisms that may be responsible for the differences. Suffice it to say that in tense utterances the spectra seem to be even flatter than in loud utterances, especially in the frequency region around $1.5 \mathrm{kHz}$. It is also of interest to note that it appears from the final multiple regression analysis, the results of which are summarized in Table 5.6, that $F_{0}$ is a better predictor of perceived tension than any slope measure, contrary to what was found for loudness. Thus either the loudness or the tension scores (or both) may depend on other acoustic parameters besides spectral slopes.

As for the discriminating power of the acoustic measures, it appeared that with syllables per second without pauses, $F_{0}$ median, $F_{0}$ variation coefficient, uncorrected $F_{0}$ perturbation, and three spectral slope measures as discriminating variables and with three discriminant functions, a discriminant analysis succeeded in assigning $43 \%$ of the emotional utterances to the right category. Of course, with ten categories to choose from, this percentage is far beyond chance, though not very impressive if compared to the classification results of the discriminant analyses based on the perceptual ratings (see Chapter 4). There it took not more than two discriminating variables, namely loudness and laxness, to yield almost the same result (38\%) as obtained here with the complete set of seven acoustic measures. With the perceptual ratings on a third variable (pitch level) added, the percentage of correct classifications was raised to $52 \%$. This differential success of the acoustic and the perceptual measures, as well as a comparison between the performance of either and the recognition by human subjects will come up for discussion in Chapter 10. There the nature of the functions extracted in the acoustically based discriminant analysis and the relationships among the emotions as they appea red from a cluster analysis on the classification matrix will also be commented on. 


\section{NOTES}

1 In fact, there was a remarkable agreement among the raters as to whether or not at a particular place in an utterance there was a pause Of the total of 19 pauses that were marked, 12 were perceived by all 6 raters, 3 by 5 raters, 1 by 3 raters, and 3 by 2 raters Equally noteworthy is the fact that of the 15 pauses that were perceived by at least 5 raters, 9 occurred in expressions of shame, which means that there were pauses in more than half of the cases in which this emotion was expressed

2 In many studies the term "roughness" is used interchangeably with "harshness"

3 Of course, intensity would have been a very likely measure as well to take as a correlate of perceived loudness and perhaps also of perceived tension However, during the recordings of the emotional expressions (see Section 224 ) and the copying of the tapes so many level adjustments had taken place that information on the original intensity of the utterances was irretrevably lost

4 Actually, the term "spectral distance" would be more accurate than "spectral slope", since "slope" implies a division by frequency distance

5 At the moment the present author is carrying out such research with the aim of assessing potential systematic differences in voice quality between speakers of different socio-economic status, regional background, and age In this research, stretches of speech with a minimum duration of 60 seconds are used 


\section{Recognition by Dutch adults}

\subsection{INTRODUCTION}

Empirical research into the recognizability of vocally expressed emotions by adults is of fairly recent date, only a few studies having been conducted before the 1950s. Since then, however, there has been a constant stream of publications devoted to the subject. Table 6.1 shows a survey of these recognition studies, listing the number and kind of encoders and decoders participating, the speech material used as the carrier for the emotional expressions, the emotional categories expressed and the rank order of recognizability, the way in which the decoders were asked to respond, and the mean percentage of correct responses obtained. Only those studies are included in the survey which examined the rate of recognition of non-manipulated emotional speech encoded by normal adults and decoded by normal adults with the same mother tongue as the encoders. Correlational studies which aimed, for example, at determining the relationship between emotional sensitivity and verbal intelligence or some other variable, have not been included. For the outcomes of studies in which synthesized or manipulated (e.g., lowpass filtered, monotonized) speech was offered for identification, we refer to Chapter 4 . The results of studies in which children served as subjects will be presented in Chapters 7 and 8 , and those of cross-cultural studies in Chapter 9.

We will only discuss here those data presented in Table 6.1 that are relevant to the setup and aims of the recognition experiment dealt with in the present chapter, i.e., the data contained in the second, third, fourth, seventh, and eighth columns; the rest of the data will come up for discussion in Section 6.4 .

From the second and third columns in Table 6.1 it appears that almost all of the recognition studies have been conducted in the United States; the recognition of vocal expressions of emotion produced and interpreted by nonEnglish speaking adults has hardly been investigated. Especially non-western languages have been neglected.

From the fourth column it appears, as has been stated in Chapter 2, that if content masking by means of random splicing, lowpass filtering, or reversed speech has not been opted for, the most common way of separating the vocal 
Table 6.1 Survey of studies on the recognition of vocal expressions of emotions by adults

\begin{tabular}{|c|c|c|c|c|c|c|c|}
\hline Study & Encoders & Decoders & Speech sample & Emotions $^{a}$ & Emotions $^{b}$ & Task & $\%$ Accuracy \\
\hline $\begin{array}{l}\text { Dusenbury \& } \\
\text { Knower, } \\
1938\end{array}$ & $\begin{array}{l}4 \mathrm{~m}, 4 \mathrm{f} \text { Am. } \\
\text { speech stud }\end{array}$ & $\begin{array}{l}\text { a) } 294 \mathrm{Am} \text { stud } \\
\text { b) } 94 \mathrm{Am} \text { stud }\end{array}$ & $\begin{array}{l}\text { letters of } \\
\text { alphabet }\end{array}$ & $\begin{array}{l}\text { contempt (3) } \\
\text { sadness (4) } \\
\text { surprise (5) } \\
\text { fear (7) } \\
\text { anger (10) }\end{array}$ & $\begin{array}{l}\text { determination (I) } \\
\text { doubt (2) } \\
\text { sympathy (6) } \\
\text { reverence (8) } \\
\text { merriment (9) } \\
\text { suffering (11) }\end{array}$ & $\begin{array}{l}\text { forced } \\
\text { judgment } \\
\text { with } 11 \\
\text { alternatives }\end{array}$ & $\begin{array}{l}\text { a) } 83 \\
\text { b) } 81\end{array}$ \\
\hline $\begin{array}{l}\text { Fairbanks \& } \\
\text { Pronovost, } \\
1939\end{array}$ & $\begin{array}{l}\text { 6m Am. } \\
\text { amateur actors }\end{array}$ & 64 Am. stud. & $\begin{array}{l}\text { standard } \\
\text { passage }\end{array}$ & $\begin{array}{l}\text { contempt (2) } \\
\text { sadness (3.5) } \\
\text { anger (3.5) } \\
\text { fear (5) }\end{array}$ & indifference (1) & $\begin{array}{l}\text { forced } \\
\text { judgment } \\
\text { with } 12 \\
\text { alternatives }\end{array}$ & 79 \\
\hline $\begin{array}{l}\text { Knower, } \\
1941\end{array}$ & $1 \mathrm{~m}$, if $\mathrm{Am}$ & Am stud & $\begin{array}{l}\text { letters of } \\
\text { alphabet }\end{array}$ & $\begin{array}{l}\text { surprise } \\
\text { lear } \\
\text { contempt } \\
\text { sadness } \\
\text { anger }\end{array}$ & $\begin{array}{l}\text { merriment } \\
\text { suffering } \\
\text { determination } \\
\text { doubt } \\
\text { sympathy } \\
\text { reverence }\end{array}$ & $\begin{array}{l}\text { forced } \\
\text { judgment } \\
\text { with } 11 \\
\text { alternatives }\end{array}$ & 89 \\
\hline $\begin{array}{l}\text { Pfaff, } \\
1954\end{array}$ & $\begin{array}{l}1 \mathrm{~m} . \text { Am speech } \\
\text { Instructor }\end{array}$ & 304 Am. stud. & numerals & $\begin{array}{l}\text { joy (2) } \\
\text { fear (2) } \\
\text { sadness (5.5) } \\
\text { disgust (5.5) } \\
\text { shame (8.5) }\end{array}$ & $\begin{array}{l}\text { hate (2) } \\
\text { doubt (5.5) } \\
\text { pleading (5.5) } \\
\text { love (8.5) } \\
\text { excitement }\end{array}$ & $\begin{array}{l}\text { forced } \\
\text { judgment } \\
\text { judgment } \\
\text { with } 10 \\
\text { alternatives }\end{array}$ & 50 \\
\hline $\begin{array}{l}\text { Davitz \& } \\
\text { Davitz, } \\
1959\end{array}$ & $\begin{array}{l}4 \mathrm{~m}, 4 \mathrm{f} \text { Am. } \\
\text { stud and fac. } \\
\text { members, } 1 \mathrm{Am} \\
\text { actress }\end{array}$ & $30 \mathrm{Am}$. stud. & $\begin{array}{l}\text { letters of } \\
\text { alphabet }\end{array}$ & $\begin{array}{l}\text { anger (1) } \\
\text { sadness (3) } \\
\text { joy (4) } \\
\text { fear (7 5) }\end{array}$ & $\begin{array}{l}\text { nervousness (2) } \\
\text { sympathy (5) } \\
\text { satısfaction (6) } \\
\text { love }(7.5) \\
\text { jealousy (9) } \\
\text { pride (10) }\end{array}$ & $\begin{array}{l}\text { forced } \\
\text { judgment } \\
\text { with } 10 \\
\text { alternatives }\end{array}$ & 37 \\
\hline
\end{tabular}




\begin{tabular}{|c|c|c|c|c|c|c|c|}
\hline Study & Encoders & Decoders & Speech sample & Emotions $^{\mathrm{a}}$ & Emotions $^{b}$ & Task & $\%$ Accuracy \\
\hline $\begin{array}{l}\text { Pollack } \\
\text { Rubenstein, \& } \\
\text { Horowitz, } \\
1960\end{array}$ & $4 \mathrm{Am}$ & $18 \mathrm{Am}$ & $\begin{array}{l}\text { standard } \\
\text { sentences }\end{array}$ & $\begin{array}{l}\text { neutral (5) } \\
\text { fear (6) } \\
\text { joy (9) } \\
\text { disgust (14) } \\
\text { surprise (15) } \\
\text { anger (16) }\end{array}$ & $\begin{array}{l}\text { pedantic (1) } \\
\text { bored (2) } \\
\text { question (3) } \\
\text { confidential (4) } \\
\text { uncertainty (7) } \\
\text { disbelıef (8) } \\
\text { impatient (10) } \\
\text { sarcasm (11) } \\
\text { threat (12) } \\
\text { approval (13) }\end{array}$ & $\begin{array}{l}\text { forced } \\
\text { judgment } \\
\text { with } \\
\text { a) } 16 \\
\text { alternatives } \\
\text { b) } 8 \\
\text { alternatives }\end{array}$ & $\begin{array}{l}\text { a) } 42 \\
\text { b) } 63\end{array}$ \\
\hline $\begin{array}{l}\text { Ljeberman \& } \\
\text { Michaels, } \\
1962\end{array}$ & $3 \mathrm{~m} \mathrm{Am}$ & $10 \mathrm{Am}$ & $\begin{array}{l}\text { standard } \\
\text { sentences }\end{array}$ & $\begin{array}{l}\text { neutral (35) } \\
\text { joy }(55) \\
\text { fear }(8)\end{array}$ & $\begin{array}{l}\text { bored (1) } \\
\text { question (2) } \\
\text { pompous (3 5) } \\
\text { disbelief (5 5) } \\
\text { confidential (7) }\end{array}$ & $\begin{array}{l}\text { forced } \\
\text { judgment } \\
\text { with } \\
8 \\
\text { alternatives }\end{array}$ & 85 \\
\hline $\begin{array}{l}\text { Beldoch, } \\
1964\end{array}$ & $3 \mathrm{~m}, 3 \mathrm{r} \mathrm{Am}$ & $\begin{array}{l}43 \mathrm{~m}, 46 \mathrm{fm} \\
\text { stud }\end{array}$ & $\begin{array}{l}\text { standard } \\
\text { passage }\end{array}$ & $\begin{array}{l}\text { disgust } \\
\text { joy } \\
\text { fear } \\
\text { anger }\end{array}$ & $\begin{array}{l}\text { love } \\
\text { admiration } \\
\text { amusement } \\
\text { boredom } \\
\text { desparr } \\
\text { impatience }\end{array}$ & $\begin{array}{l}\text { forced } \\
\text { judgment } \\
\text { with } \\
10 \\
\text { alternatives }\end{array}$ & 54 \\
\hline $\begin{array}{l}\text { Kramer, } \\
1964\end{array}$ & $\begin{array}{l}7 \mathrm{~m} \mathrm{Am} \\
\text { speech stud }\end{array}$ & $27 \mathrm{~m}$ Am stud & $\begin{array}{l}\text { standard } \\
\text { passage }\end{array}$ & $\begin{array}{l}\text { contempt (1) } \\
\text { anger (3) } \\
\text { sadness (4) }\end{array}$ & $\begin{array}{l}\text { indifference ( } 2 \text { ) } \\
\text { love (5) }\end{array}$ & $\begin{array}{l}\text { forced } \\
\text { judgment } \\
\text { with } \\
5 \\
\text { alternatives }\end{array}$ & 70 \\
\hline $\begin{array}{l}\text { Levitt, } \\
1964\end{array}$ & $\begin{array}{l}25 \mathrm{~m}, 25 \mathrm{f} \text { Am } \\
\text { stud }\end{array}$ & $\begin{array}{l}\text { 4m, 4f Am } \\
\text { stud }\end{array}$ & $\begin{array}{l}\text { standard } \\
\text { passage }\end{array}$ & $\begin{array}{l}\text { fear (1) } \\
\text { anger (2) } \\
\text { joy (3) } \\
\text { surprise (4) } \\
\text { disgust (5) } \\
\text { contempt (6) }\end{array}$ & & $\begin{array}{l}\text { forced } \\
\text { judgment } \\
\text { with } \\
6 \\
\text { alternatives }\end{array}$ & 47 \\
\hline
\end{tabular}


Table 6 I (contınued)

\begin{tabular}{|c|c|c|c|c|c|c|c|}
\hline Study & Encoders & Decoders & Speech sample & Emotions $^{2}$ & Emotions $^{b}$ & Task & $\%$ Accuracy \\
\hline $\begin{array}{l}\text { Levy, } \\
1964\end{array}$ & $3 \mathrm{~m}, 3 \mathrm{fm}$ & $\begin{array}{l}32 \mathrm{~m}, 42 \mathrm{f} \mathrm{Am} \\
\text { stud }\end{array}$ & $\begin{array}{l}\text { standard } \\
\text { passage }\end{array}$ & $\begin{array}{l}\text { disgust } \\
\text { joy } \\
\text { fear } \\
\text { anger }\end{array}$ & $\begin{array}{l}\text { love } \\
\text { admiration } \\
\text { amusement } \\
\text { boredom } \\
\text { despair } \\
\text { impatience }\end{array}$ & $\begin{array}{l}\text { forced } \\
\text { Judgment } \\
\text { with } \\
10 \\
\text { alternatives }\end{array}$ & 55 \\
\hline $\begin{array}{l}\text { Osser, } \\
1964\end{array}$ & $1 \mathrm{Am}$ actress & $80 \mathrm{Am}$ & single word & $\begin{array}{l}\text { neutral } \\
\text { joy } \\
\text { fear } \\
\text { sadness } \\
\text { anger }\end{array}$ & $\begin{array}{l}\text { love } \\
\text { boredom } \\
\text { calmness } \\
\text { enthusiasm } \\
\text { question }\end{array}$ & $\begin{array}{l}\text { forced } \\
\text { judgment } \\
\text { with } \\
10 \\
\text { alternatives }\end{array}$ & 32 \\
\hline $\begin{array}{l}\text { Hornstein, } \\
1967\end{array}$ & $\begin{array}{l}124 \mathrm{f} \text { Am } \\
\text { stud }\end{array}$ & $\begin{array}{l}124 \Gamma \text { Am } \\
\text { stud }\end{array}$ & $\begin{array}{l}\text { letters of } \\
\text { alphabet }\end{array}$ & $\begin{array}{l}\text { neutral } \\
\text { joy } \\
\text { sadness } \\
\text { anger }\end{array}$ & $\begin{array}{l}\text { affection } \\
\text { boredom } \\
\text { cheerfulness } \\
\text { impatience } \\
\text { satisfaction }\end{array}$ & $\begin{array}{l}\text { forced } \\
\text { Judgment } \\
\text { with } \\
9 \\
\text { alternatives }\end{array}$ & 59 \\
\hline $\begin{array}{l}\text { Heike, } \\
1969\end{array}$ & 10 Germans & Germans & $\begin{array}{l}\text { standard } \\
\text { sentence }\end{array}$ & $\begin{array}{l}\text { anger (2) } \\
\text { fear (3) } \\
\text { joy (4 5) }\end{array}$ & $\begin{array}{l}\text { reassuring (1) } \\
\text { Ironic (4 5) } \\
\text { indifference (6) }\end{array}$ & $\begin{array}{l}\text { free } \\
\text { choice }\end{array}$ & 68 \\
\hline $\begin{array}{l}\text { Plaikner, } \\
1970\end{array}$ & $\begin{array}{l}1 \text { German } \\
\text { actor }\end{array}$ & $\begin{array}{l}30 \mathrm{~m}, 19 \mathrm{f} \\
\text { Germans }\end{array}$ & $\begin{array}{l}\text { letters of } \\
\text { alphabet }\end{array}$ & $\begin{array}{l}\text { joy (2) } \\
\text { fear (3) } \\
\text { sadness (5) } \\
\text { anger }(65) \\
\text { disgust }(105)\end{array}$ & $\begin{array}{l}\text { bored }(1) \\
\text { hate }(4) \\
\text { Impatience }(65) \\
\text { enthusiasm (8) } \\
\text { despair }(9) \\
\text { benevolent }(105) \\
\text { recklessness }(125) \\
\text { longng }(125) \\
\text { love (14) } \\
\text { timidity (15) }\end{array}$ & $\begin{array}{l}\text { forced } \\
\text { Judgment } \\
\text { with } \\
\text { 15 } \\
\text { alternatives }\end{array}$ & 53 \\
\hline
\end{tabular}


Table 6.1 (contınued)

\begin{tabular}{|c|c|c|c|c|c|c|c|}
\hline Study & Encoders & Decoders & Speech sample & Emotions $^{\mathrm{a}}$ & Emotions & Task & \% Accuracy \\
\hline $\begin{array}{l}\text { Bluhme, } \\
1971\end{array}$ & $\begin{array}{l}\text { 6m French } \\
\text { stud. }\end{array}$ & $\begin{array}{l}\text { 9f French stud. } \\
\text { and teachers of } \\
\text { phonetics }\end{array}$ & $\begin{array}{l}\text { standard } \\
\text { sentences }\end{array}$ & $\begin{array}{l}\text { anger (1) } \\
\text { neutral (3) } \\
\text { contempt (7) } \\
\text { surprise ( } 8 \text { ) }\end{array}$ & $\begin{array}{l}\text { hesitation (2) } \\
\text { supposition (4) } \\
\text { question (5) } \\
\text { irony (6) } \\
\text { conviction (9) } \\
\text { admiration (10) } \\
\text { subj. statement (11) } \\
\text { disapproval (12) } \\
\text { tag-question (13) }\end{array}$ & $\begin{array}{l}\text { forced } \\
\text { judgment } \\
\text { with } \\
13 \\
\text { alternatives }\end{array}$ & 33 \\
\hline $\begin{array}{l}\text { Wolf, } \\
\text { Gorskı, \& } \\
\text { Peters, } \\
1972\end{array}$ & $\begin{array}{l}25 m \text { Am. } \\
\text { stud. }\end{array}$ & $25 \mathrm{~m}$ Am stud. & $\begin{array}{l}\text { letters of } \\
\text { alphabet }\end{array}$ & $\begin{array}{l}\text { joy } \\
\text { fear } \\
\text { sadness } \\
\text { anger }\end{array}$ & $\begin{array}{l}\text { Jealousy } \\
\text { love } \\
\text { nervousness } \\
\text { pride } \\
\text { satisfaction } \\
\text { sympathy }\end{array}$ & $\begin{array}{l}\text { forced } \\
\text { Judgment } \\
\text { with } \\
10 \\
\text { alternatives }\end{array}$ & 45 \\
\hline $\begin{array}{l}\text { Burns \& } \\
\text { Beier, } \\
1973\end{array}$ & $\begin{array}{l}30 \mathrm{Am} . \\
\text { stud }\end{array}$ & 21 Am. stud. & $\begin{array}{l}\text { standard } \\
\text { sentences }\end{array}$ & $\begin{array}{l}\text { anger (1) } \\
\text { sadness (2) } \\
\text { joy (5) }\end{array}$ & $\begin{array}{l}\text { anxious (3) } \\
\text { indifferent (4) } \\
\text { seductive (6) }\end{array}$ & $\begin{array}{l}\text { forced } \\
\text { judgment } \\
\text { with } 7 \\
\text { alternatives }\end{array}$ & 60 \\
\hline $\begin{array}{l}\text { Ross, } \\
\text { Duffy, } \\
\text { Cooker, \& } \\
\text { Sergeant, } \\
1973\end{array}$ & $\begin{array}{l}\text { 3m, 3f Am. } \\
\text { actors }\end{array}$ & 33 Am. stud. & $\begin{array}{l}\text { standard } \\
\text { passage }\end{array}$ & $\begin{array}{l}\text { anger (1) } \\
\text { sadness (3) } \\
\text { fear (6) } \\
\text { contempt (7.5) } \\
\text { surprise (9) }\end{array}$ & $\begin{array}{l}\text { Indifference (2) } \\
\text { amusement (4) } \\
\text { doubt (5) } \\
\text { love (7.5) }\end{array}$ & $\begin{array}{l}\text { forced } \\
\text { Judgment } \\
\text { with } \\
9 \\
\text { alternatives }\end{array}$ & 70 \\
\hline $\begin{array}{l}\text { Theyssen, } \\
1973\end{array}$ & $\begin{array}{l}2 \mathrm{~m}, 2 f \text { Dutch } \\
\text { stud. }\end{array}$ & $\begin{array}{l}4 \mathrm{~m}, 6 \mathrm{f} \text { Dutch } \\
\text { stud. }\end{array}$ & $\begin{array}{l}\text { standard } \\
\text { sentences }\end{array}$ & $\begin{array}{l}\text { sadness (3) } \\
\text { joy (4) }\end{array}$ & $\begin{array}{l}\text { question (1) } \\
\text { indigant (2) } \\
\text { conspiratory (5) } \\
\text { apologizing (6) }\end{array}$ & $\begin{array}{l}\text { a) forced } \\
\text { judgment with } \\
6 \text { alternatives } \\
\text { b) free choice }\end{array}$ & $\begin{array}{l}\text { a) } 70 \\
\text { b) } 51\end{array}$ \\
\hline
\end{tabular}


Table $6 /$ (contunued)

\begin{tabular}{|c|c|c|c|c|c|c|c|}
\hline Study & Encoders & Decoders & Speech sample & Emotions $^{a}$ & Emotions $^{b}$ & Task & $\%$ Accuracy \\
\hline $\begin{array}{l}\text { Zuckerman, } \\
\text { Lıpets, } \\
\text { Koıvumakı, \& } \\
\text { Rosenthal, } \\
1975\end{array}$ & $\begin{array}{l}27 \mathrm{~m}, 13 f \mathrm{Am} \\
\text { stud }\end{array}$ & $\begin{array}{l}\text { a) } 64 \mathrm{~m} \mathrm{Am} \text { stud } \\
\text { b) } 37 \mathrm{f} \mathrm{Am} \mathrm{stud}\end{array}$ & $\begin{array}{l}\text { standard } \\
\text { sentence }\end{array}$ & $\begin{array}{l}\text { sadness (1) } \\
\text { surprise (2) } \\
\text { anger (3) } \\
\text { disgust (4) } \\
\text { joy (5) } \\
\text { fear (6) }\end{array}$ & & $\begin{array}{l}\text { forced } \\
\text { judgment } \\
\text { with } 6 \\
\text { alternatives }\end{array}$ & $\begin{array}{l}\text { a) } 45 \\
\text { b) } 46\end{array}$ \\
\hline $\begin{array}{l}\text { Fenster, } \\
\text { Blake, \& } \\
\text { Goldstein, } \\
1977\end{array}$ & $30 \mathrm{~m} \mathrm{Am}$ & $30 \mathrm{Am}$ & $\begin{array}{l}\text { standard } \\
\text { sentence }\end{array}$ & $\begin{array}{l}\text { sadness (1) } \\
\text { anger (2) } \\
\text { fear (4) } \\
\text { joy (6) }\end{array}$ & $\begin{array}{l}\text { love (3) } \\
\text { contentment (5) }\end{array}$ & $\begin{array}{l}\text { forced } \\
\text { judgment } \\
\text { with } 6 \\
\text { alternatives }\end{array}$ & 30 \\
\hline $\begin{array}{l}\text { Brown, } \\
1980\end{array}$ & $\begin{array}{l}5 \text { Spanısh bı } \\
\text { linguals with } \\
\text { English as } \\
\text { their first } \\
\text { language }\end{array}$ & $18 \mathrm{Am}$ stud & $\begin{array}{l}\text { film } \\
\text { description }\end{array}$ & $\begin{array}{l}\text { disgust } \\
\text { surprise } \\
\text { joy } \\
\text { fear } \\
\text { contempt } \\
\text { sadness } \\
\text { anger }\end{array}$ & $\begin{array}{l}\text { depression } \\
\text { hate } \\
\text { humor } \\
\text { love } \\
\text { pity } \\
\text { reverence }\end{array}$ & $\begin{array}{l}\text { forced } \\
\text { Judgment } \\
\text { with } 13 \\
\text { alternatives }\end{array}$ & - \\
\hline $\begin{array}{l}\text { Williams \& } \\
\text { Stevens, } \\
1981\end{array}$ & $\begin{array}{l}3 \mathrm{~m} \mathrm{Am} \\
\text { actors }\end{array}$ & $12 \mathrm{Am}$ & $\begin{array}{l}\text { standard } \\
\text { sentences }\end{array}$ & $\begin{array}{l}\text { sorrow (1) } \\
\text { anger (2) } \\
\text { neutral (3) } \\
\text { happy (4) } \\
\text { fear (5) }\end{array}$ & & $\begin{array}{l}\text { forced } \\
\text { judgment } \\
\text { with } 5 \\
\text { alternatives }\end{array}$ & 45 \\
\hline
\end{tabular}

Note The numbers in parentheses indicate the rank order in which the emotions were recognized, from $(1)=$ best to $(n)=$ worst

a emotions also present in our study

b emotions not present in our study

\% Accuracy $=$ mean percentage of correct responses

$\mathrm{m}=$ male

f = female

stud $=$ students

Am = American Englısh 
from the verbal communication channel has been to keep the verbal content constant over all emotional utterances so that only vocal features of speech can vary. In this manner one can be sure that it was not what the speaker said but how he or she said it that permitted the transmission of whatever emotional meaning got across. As has been argued in Chapter 2, we think that the best type of standard speech sample is meaningful speech material in the form of phrases or sentences.

The data in the seventh column of Table 6.1 reveal that in most studies the subjects were given a fixed set of alternatives from which to select the one best fitting the emotional portrayal presented. Usually this was the same list of categories which the speakers had employed when producing the portrayals. Free choice tasks, i.e., tasks in which the subjects were asked to describe the stimuli in their own words, have been fairly rare, probably because they involve the methodological problem of deciding which responses are correct and which ones are not. Heike (1969) does not mention how this problem was dealt with in his study; he merely states that the number of correct identifications was assessed by summing the responses which were identical with or similar to the expression categories. How similarity was defined he does not say.

Theyssen (1973) used both a forced judgment and a free choice task for determining the recognizability of her stimuli. In the free choice session a total of 115 descriptive labels were used. These were reassigned to the original expression categories by an independent group of subjects. The responses obtained by the two procedures manifested similar structures, both when subjected to a hierarchical cluster analysis and when processed by means of a multidimensional scaling technique (Bouwhuis, 1974). However, it appeared that the average recognition rate was considerably lower for the free choice task than for the forced judgment task (51\% vs. $70 \%$ ), a consequence of the "noise" introduced by the reassignment of the labels used by the listeners to the labels used by the speakers.

In the eighth and last column of Table 6.1 the mean percentage of correct identifications is given for each study. The average accuracy across all studies included in the survey is $57 \%$. This is much higher than is to be expected by chance since in most studies the subjects had to choose from six to twelve alternatives, which corresponds with an accuracy to be expected by chance of between $8 \%$ and $17 \%$. The average accuracy corrected for chance is $51 \%$, a figure which should be compared with an average expected chance percentage of about $12 \%$. Thus, it appears that in general vocally expressed emotions are recognizable well beyond chance.

An equally general finding, however, and one that is not apparent from the survey, has been that the recognizability of different emotions varies considerably (a significant effect of the factor emotion is reported, for example, in Burns \& Beier, 1973; Pfaff, 1954; Reid \& Ware, 1971). Since, as we have remarked before, almost all recognition data pertain to English expressions, we thought it interesting to determine whether the same finding 
would emerge from a study of Dutch emotional expressions, and, more importantly, to see to what extent the differences in recognizability found for Dutch are comparable to those which have previously been reported for English.

It is also commonly reported that the rate of recognition may be affected by a number of subject variables. Two of these variables, namely cultural distance between speaker and listener and age, will be considered in other chapters. In the present chapter the effect of the sex of the speaker and the sex of the listener will be examined.

As for the sex of the speaker mostly nonsignificant differences have been reported (Dusenbury \& Knower, 1938; Levitt, 1964; Levy, 1964; Zuckerman, Lipets, Koivumaki, \& Rosenthal, 1975). Only Kozel and Gitter (cited in Fenster, Blake, \& Goldstein, 1977) report a female advantage. These data all pertain to American English research.

The results with respect to the sex of the listener are quite comparable. Hall (1978) summarizes the results of seventy-five - American English - studies reporting accuracy for males and females at decoding nonverbal communication in three modalities, namely facial, vocal, and facial and vocal combined. Although on the whole females do better than males, it appears that the size of the gender effect depends on the modality investigated. The superior performance of the women is most striking in the facial-plus-vocal channel, and least obvious in the vocal-only studies. Out of the ten studies in which accuracy of adult males and females in identifying vocal expressions of emotion is compared, three indicate a significant female advantage and seven show no significant differences between the two sexes.

Thus, in most American English studies on the recognition of vocally expressed emotion no significant effect of the sex of the speaker nor of the sex of the listener has been found. Very little is known, however, about the influence of both the sex of the speakers and the sex of the listeners on the identification of non-English expressions of emotion.

A problem which in our opinion has been unjustly neglected - unjustly because it may have consequences for the validity of recognition results - is the question of whether the rate of recognition varies as a function of the speech material employed. This factor will therefore also be considered. In short, the present study aimed at answering the following questions:

(1) Is there a difference in the recognizability of the different emotions examined?

(2) Is there a difference between males and females in the accuracy with which they identify vocal expressions of emotion?

(3) Is there a difference between males and females in the clearness with which they convey emotional expressions?

(4) Is the rate of recognition affected by the phrase with which the emotions are expressed? 


\subsection{METHOD}

Recognition was assessed by means of a forced judgment task in which the subjects responded to vocal expressions of emotion by choosing among emotion labels.

\subsubsection{Subjects}

Forty-eight subjects, twenty-four males and twenty-four females, took part in the experiment. All were language students attending a Teachers' Training College in Nijmegen. Their ages ranged between 18 and 28 years, with a mean of 20.6 years. The subjects were paid for their participation.

\subsubsection{Speech material}

The speech material consisted of the complete set of 320 stimuli, namely eight speakers (four males and four females) $X$ four phrases (daar is ie weer, twee maanden zwanger, ze is bij de luchtmacht, zo'n grote Amerikaanse auto) $\times$ ten emotions (neutral [NE], disgust [DI], surprise [SU], shame [SH], interest [IN], joy [JO], fear [FE], contempt [CO], sadness [SA], anger [AN]). (For more information on the stimuli, see Chapter 2.)

\subsubsection{Procedure}

The experiment took place in a language laboratory (type Tandberg Teaching System IS 6). Since the laboratory was too small to accommodate all subjects at once, they were randomly divided over two groups, either group, however, containing an equal number of males and females. After the subjects had been seated, each in a separate listening booth, they were first offered the four phrases used to express the various emotions in written form with the request to indicate with which of the ten emotions they associated their verbal content. (For the exact formulation of the instructions for the association task, see Appendix B.)

After the subjects had finished the association task, they heard six practice stimuli in order to get acquainted with the recognition task. When it was clear that the task was understood, the 320 experimental stimuli were played in four blocks of eighty stimuli which had each been put in a random order, separately for the male and the female speakers. One group of subjects started with a block of female expressions, followed by a block of male expressions, a block of female expressions, and a block of male expressions. For the other group of subjects the order was reversed. An interstimulus interval of 8 seconds allowed the subjects to note down which of the ten emotions they thought had been expressed. The subjects were told that any emotion could appear any number of times. (For the exact formulation of the instructions for the recognition experiment, see Appendix B.) 
The total duration of the experiment was two hours, including three breaks of 10 minutes each between the blocks.

\subsection{RESULTS}

In Table 6.2 the results are presented of the association task. The data in this table reveal that there are marked differences in the frequency with which the verbal contents of the different phrases evoked associations with specific emotional categories. Daar is ie weer was associated most often with disgust, surprise, joy, and contempt; twee maanden zwanger with neutral and joy; ze is bij de luchtmacht with neutral and interest; and zo'n grote Amerikaanse auto with disgust and contempt.

In order to determine whether the two orders in which the stimuli had been presented had had an effect on the recognition scores, a $t$-test was performed on the mean number of correct responses per emotion for either condition. No significant difference was found. Therefore, it was decided to make use of the combined data in the further analyses.

In order to answer the four questions formulated in the introduction, a $2 \times 4$ $\times 10 \times 2 \times 48$ analysis of variance (repeated measurements design) was carried out with four fixed factors, namely sex of speaker, phrase, emotion, and sex of subject, and one random variable, namely subjects. Subjects were nested under the factor sex of subject (for details about the design, see note 1). Input to the program were the numbers of correct identifications for each emotion, summed over the individual male and summed over the individual female speakers; the input data ranged between 0 and 4 . The level of significance was set at $5 \%$.

\subsubsection{Factor emotion}

It appeared that the first question, whether there is a difference in the recognizability of the different emotions examined, has to be answered positively, since there was a significant effect of the factor emotion $(F(9,414)$

Table 6.2 Frequency with which the verbal contents of four phrases are associated with ten emotions

\begin{tabular}{lrrrrrrrrrr}
\hline & NE & DI & SU & SH & IN & JO & FE & CO & SA & AN \\
\hline Daar is ie weer & 2 & 12 & 8 & 0 & 2 & 8 & 1 & 12 & 0 & 3 \\
Twee maanden zwanger & 14 & 0 & 6 & 2 & 6 & 14 & 2 & 3 & 1 & 0 \\
Ze is bij de luchtmacht & 22 & 5 & 4 & 0 & 11 & 0 & 0 & 6 & 0 & 0 \\
Zo'n grote Amerikaanse auto & 4 & 16 & 5 & 0 & 6 & 2 & 0 & 15 & 0 & 0 \\
Total & 42 & 33 & 23 & 2 & 25 & 24 & 3 & 36 & 1 & 3 \\
\hline
\end{tabular}


$=48.00, p<.001$ ). In the first column of Table 6.3 the uncorrected percentages of correct identification for each of the ten emotions are given. Although a binomial estimate of probability revealed that all emotional categories were recognized above chance expectancy $(p<.05)$, it may clearly be seen that some emotions, such as surprise, joy, and anger, were identified considerably better than others, such as disgust, shame, and contempt. Neutral, which is not to be considered as a real emotion, yielded by far the greatest number of correct responses.

Inspection of the values in the second column of Table 6.3 reveals, however, that not all emotional categories appear as responses equally often, i.e., the subjects have manifested a response bias: Neutral, surprise, and contempt have been chosen considerably more often than average, whereas disgust, shame, interest, and fear have been chosen considerably less often

Table 6.3 Recognition by Dutch adults: Mean percentages of correct identification, both uncorrected and corrected for chance as well as for response bias, and mean percentages of responses

\begin{tabular}{llcll}
\hline & Uncorrected & Responses & $\begin{array}{l}\text { Corrected } \\
\text { for chance }\end{array}$ & $\begin{array}{l}\text { Corrected for } \\
\text { response bras }\end{array}$ \\
\hline Neutral & $86(1)$ & 117 & 84 & $80(1)$ \\
Disgust & $49(10)$ & 84 & 43 & $54(9)$ \\
Surprise & $69(4)$ & 127 & 66 & $62(7.5)$ \\
Shame & $54(8.5)$ & 75 & 49 & $63(6)$ \\
Interest & $63(6)$ & 87 & 59 & $68(4)$ \\
Joy & $72(3)$ & 99 & 69 & $72(3)$ \\
Fear & $58(7)$ & 88 & 53 & $62(7.5)$ \\
Contempt & $54(8.5)$ & 121 & 49 & $49(10)$ \\
Sadness & $67(5)$ & 101 & 63 & $67(5)$ \\
Anger & $74(2)$ & 100 & 71 & $74(2)$ \\
Grand mean & 65 & 100 & 61 & 65 \\
\hline
\end{tabular}

Note. The numbers in parentheses indicate the rank order positions from $1=$ best to $10=$ worst recognized emotion.

a The percentages corrected for chance have been calculated by means of the formula:

$$
p=\frac{p(c)-1 / n}{1-1 / n} \text { in which }
$$

$\mathrm{p}=\%$ correct corrected for chance

$\mathrm{p}(\mathrm{c})=\%$ correct observed

$n=$ number of alternatives

b The percentages corrected for response bias have been calculated by means of the formula:

$$
p=\frac{p(c)+p(c) / p(r)}{2} \text { in which }
$$

$\mathrm{p}=\%$ correct corrected for response bias

$p(c)=\%$ correct observed

$p(r)=\%$ responses 
than average. The bias of choosing a particular category too often tends to artificially enlarge accuracy for the biased category; the bias of choosing a particular category not often enough has the opposite effect.

In the third column of Table 6.3 the percentages corrected for chance (see note a) are given and in the fourth column the percentages corrected for response bias (see note b). The former correction has the advantage of enhancing to some degree the comparability of results stemming from experiments with unequal numbers of response categories.

Finally, in Table 6.3 the rank orders of the recognition of the different emotions, based on the raw scores as well as the scores corrected for response bias, are also indicated (the rank order of the percentages which have been corrected for chance have been omitted since with this correction the original rank order of the raw scores is preserved). It may be seen that the correction for response bias has either no or not more than a minor effect on the rank order positions of most emotions. Exceptions are the rank order positions of surprise and shame, the two emotional categories which were characterized by the most extreme response biases, surprise dropping from the fourth to the seventh-and-a-half position, and shame rising from the eighth-and-a-half to the sixth position.

\subsubsection{Factors sex of speaker and sex of subject}

The results of the analysis of variance further revealed that there was no significant effect of the sex of the speakers, the sex of the subjects, and the interaction between the sex of the speakers and the sex of the subjects on the scores $(F(1,46)=2.11 p>.05, F(1,46)=2.60, p>.05$, and $F(1,46)=0.69$, $p>.05$, respectively). This means that the second and third questions have to be answered in the negative: There was no difference between males and females in the clearness with which they conveyed emotional expressions, and there was no difference between males and females in the accuracy with which they identified vocal expressions of emotion.

\subsubsection{Factor phrase}

The results of the analysis of variance finally revealed that the fourth question, whether the rate of recognition depended on the phrase with which the various emotions were expressed, has to be answered positively $(F(3,138)$ $=49.68, p<.001)$. Inspection of the cell means showed that on the average the emotions expressed with the phrase daar is ie weer were recognized considerably less well than those expressed with the other three phrases, i.e., twee maanden zwanger, ze is bij de luchtmacht, and zo'n grote Amerikaanse auto, the mean percentages of correct identification being 58,66,67, and 68 , respectively.

The second order interactions phrase $X$ sex of subject, phrase $X$ sex of speaker, and phrase $X$ emotion yielded significant effects as well $(F(1,138)=$ 
$3.04, p<.05, F(3,138)=8.68, p<.01$, and $F(27,1242)=15.86, p<.001$, respectively). In all three cases this was mainly due to the "deviant" behaviour of the phrase daar is ie weer: While for the other three phrases there was virtually no difference between male and female subjects in the number of correct responses, and while for those phrases it did not make much difference whether they had been produced by a male or a female speaker, with respect to daar is ie weer the female subjects did better than the male subjects (a mean of $60 \%$ vs. $55 \%$ ) and the male speakers did better than the female speakers (a mean of $61 \%$ vs. $54 \%$ ). As to the interaction between phrase and emotion it appeared that with daar is ie weer the recognizability of expressions of surprise, shame, interest, and sadness was affected negatively, whereas the recognizability of expressions of disgust and fear was affected positively in comparison with the rate of recognition of these emotions when expressed with the other phrases.

\subsection{DISCUSSION}

\subsubsection{Factor emotion}

The present study revealed that although all emotions examined were recognized above chance expectancy, some were considerably easier to identify than others. If the uncorrected percentages of correct identification are considered, it appears that the recognition of neutral, surprise, joy, and anger was relatively easy, whereas the recognition of disgust, shame, and contempt was relatively difficult. If the percentages corrected for response bias are considered, a slightly different picture presents itself. Then shame is identified correctly more often than surprise. This outcome makes clear that if the response bias is extreme enough, it may have a considerable effect on the number of correct identifications obtained. This factor should be taken into account when interpreting results from the literature.

It is impossible to explain the response biases found in the present study. They are not significantly correlated with the frequency with which the verbal contents of the four phrases (see bottom row Table 6.2) were associated with the various emotions $(r(8)=.47, p>.10)$. Neither can the biases we have found be accounted for by an occasionally reported tendency of subjects to interpret emotional expressions negatively (Dimitrovsky, 1964; Hornstein, 1967), the categories which in our experiment have been chosen most often being neutral, surprise, and contempt.

For determining to what extent our results as to the relative recognizability of the different emotions correspond with earlier reported data, the percentages of correct identification, either uncorrected, corrected for response bias, or corrected for chance are not a very good basis. As for the uncorrected percentages, their unsuitability for this purpose has already been argued 
above. A comparison cannot be based on percentages corrected for response bias since this correction has hardly ever been applied in other studies and since the data necessary for its computation are rarely provided. Moreover, by comparing percentages corrected for response bias, the fact, for example, that the number of response categories is an important factor in determining the significance of the number of correct responses, is not taken into account. The latter factor is taken into consideration if one compares percentages which have been corrected for chance. However, the fact that it is not only the number of alternatives but also the specific combination of alternatives which plays a role - the more the alternatives are alike the more difficult the choice of the right response - is impossible to compensate for since there is no objective criterion for assessing the degree of similarity between response categories nor an objective measure for expressing it. Moreover, there are many other variables which may be expected to affect the percentages of correct identification and therewith hamper comparison, such as the manner in which the stimuli were obtained (was there a selection or not, and if so, on the basis of what criteria), the type of encoders (trained or not), and the recognition task (free choice vs. forced choice).

By comparing the rank orders in which the emotions were recognized instead of the percentages correct identification, some but not all of the difficulties mentioned above are avoided. It remains problematic, for example, that reported rank orders are never corrected for response bias. Also, the rank order positions of particular categories may still be affected by the presence or absence of specific other categories. Thus, it is to be expected that disgust and contempt will each end up higher in the rank order if they occur alone than if they occur together in an experiment, since in the latter case their rates of recognition will probably be lowered by mutual confusions. Also, the general problem remains that in almost every study a different set of emotions has been examined (in the twenty-four studies included in the survey, a total of sixty-three different categories may be found); no study reporting rank orders or data from which rank orders may be deduced, has more than six emotions in common with the present study. Another problem is that not all emotions have been investigated equally often. Joy, fear, sadness, and anger have been studied relatively frequently, each figuring in at least fifteen studies. Neutral, disgust, surprise, and contempt have been included in at least six studies. Shame, however, has been looked at only once, and interest never. Because of all these complications not more than a partial and very rough comparison between our results and those obtained in other studies is possible.

In the fifth column of Table 6.1 the rank order positions are given separately for those emotions which other experiments and ours have in common. Inspection of the data shows that of the eight categories figuring in more than one study (as mentioned above, shame and interest have hardly ever or never been looked at) only the recognition of neutral and disgust manifests a more or less consistent pattern, neutral (four studies) generally 
occupying a high rank order position (because the distinction between non-emotional and emotional speech is of a fundamentally different nature than the way in which samples of specific emotional categories are distinguished?) and disgust (five studies) generally occupying a low rank order position. These outcomes agree with ours. There appears to be some pattern, though less consistent, in the rank order positions of joy and sadness also, joy (eleven studies) usually being situated in the middle or towards the lower end of the rank order (in our study it occupies the third position), and sadness (twelve studies) usually being situated in the middle or towards the higher end of the rank order (in our study it occupies the fifth position). The results for the other four emotions are impossible to summarize. This may be illustrated with what has been reported on the recognizability of anger, which appeared to be the best recognized primary emotion in five studies and the worst recognized primary emotion in three studies (in six studies it occupied an intermediate position). In our own study anger is the second best recognized emotion, after neutral. The situation for surprise, fear, and contempt is comparable.

It is difficult to estimate which factors are responsible for this lack of agreement. Some of the variables mentioned above have certainly played a role. Their relative importance, however, can only be guessed. In order to gain insight into this problem it would be of great help already if in future research more attention were paid to the choice of the emotional categories investigated, for example by concentrating on the so-called primary emotions. Restriction, for the time being, to a limited set of primary emotions would also facilitate the comparison with the outcomes of research into the recognition of facially expressed emotions. It would furthermore be helpful if response biases were reported so that possible consistencies in their occurrence, and their effect on recognition rates, could be better studied.

\subsubsection{Factors sex of speaker and sex of subject}

In the present study no significant difference was found between males and females in the clearness with which they conveyed emotional meaning. This finding is consistent with the outcomes of most research in which this question has been considered (Dusenbury \& Knower, 1938; Levitt, 1964; Levy, 1964; Zuckerman et al., 1975). No difference was found in the accuracy with which males and females were able to perceive emotional meaning either. This finding agrees with the outcomes of most relevant research as well (for an overview, see Hall, 1978). Thus, the traditional and prescientific idea that women would be superior in the expression and perception of emotional meaning is not confirmed by the results of empirical research into this question. 


\subsubsection{Factor phrase}

The finding that on the whole the emotions expressed with daar is ie weer were recognized significantly less well than those expressed with the other three phrases, is not easy to explain. It could be hypothesized that the relative lengths of the phrases have somethıng to do with it, daar is ie weer being the shortest one of the four, but then the finding that for two emotions this phrase yielded the highest number of correct responses remains unaccounted for.

It could also be that the verbal content of daar is ie weer was less neutral or evoked a stronger association with a few particular emotions than the other phrases, thus perhaps hindering the recognition of some emotions and facilitatıng the recognition of others. The association scores given in Table 6.2 reveal that the subjects did not consider the verbal contents of the four phrases to be equally neutral. The data suggest, however, that it is not because daar is te weer was verbally less neutral that it was recognized in a different manner, the difference in neutrality with $z 0^{\circ} n$ grote Amerikaanse auto being extremely small. In order to determine whether there was a relationship between the percentages of tımes that the verbal content of daar is ie weer was associated with a particular emotion and the percentages of times that this phrase was correctly identified as the expression of that emotion, a product-moment correlation was computed between the two. The correlation was extremely low $(r=.15)$. This outcome suggests that the answer as to the cause of the deviant recognition behaviour of daar is te weer cannot be found in a specific emotional colouring of its verbal content either. Not being able to thınk of another explanation, we are forced to admit that the problem of why this phrase behaved differently than the others remains unsolved.

Since we are not the only ones who have found an unexplainable effect of the factor phrase (see, for example, Pollack, Rubensteın, \& Horowitz, 1960), in future research the speech material used as the carrier for the emotional expressions had better not be restricted to one or two phrases. If the number is high enough, the differential effect of the varıous phrases will hopefully be averaged out; if not, one could consider discarding the deviant one(s). At the same time, the problem in itself is intriguing enough to deserve further attention.

NOTE

1 The factor sex of subject (I) was tested against subjects within groups, sex of speaker (J) against $J \times$ subj $w$ gr, phrase $(K)$ against $K \times$ subj $w$ gr, emotion $(L)$ against $L \times$ subj $w$ gr , I $\times J$ against $J \times$ subj $w$ gr,$I \times K$ against $K \times$ subj $w$ gr , I $X$ Lagainst $L X$ subj $w . g r, J \times K$ against $\mathrm{J} \times \mathbf{K} \times$ subj $w$ gr, $\mathrm{J} \times \mathrm{L}$ against $\mathrm{J} \times \mathrm{L} \times$ subj $\mathbf{w}$ gr, $\mathrm{K} \times \mathrm{L}$ against $\mathrm{K} \times \mathbf{L} \times$ subj $\mathbf{w}$. gr , I $\times \mathrm{J} \times \mathrm{K}$ against $\mathrm{J} \times \mathrm{K} \times$ subj $\mathbf{w}$ gr ; I $\times \mathrm{J} \times \mathrm{L}$ against $\mathrm{J} \times \mathbf{L} \times$ subj. $w$ gr , I $\times \mathbf{K} \times \mathbf{L}$ against $K \times L \times$ subj $w$ gr,$J \times K \times L$ against $J \times K \times L \times$ subj $w$ gr , and, finally, $I \times J \times K \times$ L against $J \times K \times L \times$ subj $w$ gr 


\section{Developmental recognition}

\subsection{INTRODUCTION}

Chapter 6 dealt with the recognition of vocal expressions of emotion by Dutch adults. In the present chapter the developmental recognition by Dutch children age $5 \frac{1}{2}$ to $11 \frac{1}{2}$ is considered.

The first and for a long time only empirical study dealing with the development of the recognition of vocally expressed emotions during childhood dates from 1927 when Gates tested the ability of 627 American children from grades 3 through 8 to identify expressions of surprise, happiness, fear, scorn, anger, defiance, pity, suspicion, and unhappiness produced by one actress while reciting the letters of the alphabet. Unfortunately, only a brief report of this study was published, so very little is known about the results. It was not until 1964 that the issue was reopened. In that year Dimitrovsky conducted an experiment with a total of 224 children. From that time on every couple of years a study on the developmental recognition of vocal expressions of emotion has been reported.

Table 7.1 shows a survey of these studies, listing the number and kind of encoders and decoders participating, the speech material used as the carrier for the emotional expressions, the number of stimuli offered, the emotional categories expressed, and the manner in which the decoders were asked to respond. Only those studies have been included in the survey which examined the rate of recognition of vocal expressions of emotion, either in their original form or content masked, encoded by adults or children with the same mother tongue as the decoders. Of those studies in which the relative recognizability of emotional expressions in different communication channels was examined, only the data pertaining to the vocal channel are given. For the - rare - developmental studies in a cross-cultural setting, we refer to Chapter 9.

It appears from the second and third columns in Table 7.1 that almost all of the developmental recognition studies have been conducted in the United States, i.e., hardly anything is known about the recognition of non-American emotional expressions by non-American children, a situation which is comparable to the state of affairs for the recognition by adults (see Section 6.1). It may also be seen that most children whose ability to identify emotional expressions was examined, were older than 7 years. 
Table $7 /$ Survey of studies on the recognition of vocal expressions of emotions by children

\begin{tabular}{|c|c|c|c|c|c|c|c|}
\hline Study & Encoders & Decoders & Speech sample & $\begin{array}{l}\text { Number of } \\
\text { stımuls }\end{array}$ & Emotions $^{\mathbf{a}}$ & Emotions $^{b}$ & Task \\
\hline $\begin{array}{l}\text { Gates, } \\
1927\end{array}$ & $\begin{array}{l}1 \text { Am. } \\
\text { actress }\end{array}$ & $\begin{array}{l}627 \text { Am children } \\
\text { age } 8 \text { to } 13\end{array}$ & $\begin{array}{l}\text { letters of } \\
\text { alphabet }\end{array}$ & 9 & $\begin{array}{l}\text { surprise } \\
\text { joy } \\
\text { fear } \\
\text { contempt } \\
\text { anger }\end{array}$ & $\begin{array}{l}\text { defiance } \\
\text { pity } \\
\text { suspicion } \\
\text { unhappiness }\end{array}$ & $?$ \\
\hline $\begin{array}{l}\text { Dimitrovsky, } \\
1964\end{array}$ & $\begin{array}{l}\text { f Am.adults, } \\
\text { m Am. adults }\end{array}$ & $\begin{array}{l}14 \mathrm{~m} \text { Am. and } 14 \Gamma \\
\text { Am. children } \\
\text { age } 5 \text { to } 12\end{array}$ & $\begin{array}{l}\text { standard } \\
\text { passage }\end{array}$ & 24 & $\begin{array}{l}\text { joy } \\
\text { sadness } \\
\text { anger }\end{array}$ & love & $\begin{array}{l}\text { forced } \\
\text { judgment } \\
\text { with } 4 \text { stick } \\
\text { figure } \\
\text { drawings }\end{array}$ \\
\hline $\begin{array}{l}\text { Fenster, } \\
1967\end{array}$ & $\begin{array}{l}\text { Am. children, } \\
\text { Am. adults }\end{array}$ & $\begin{array}{l}\text { Am. children } \\
\text { Am. adults }\end{array}$ & $?$ & $?$ & $\begin{array}{l}\text { joy } \\
\text { fear } \\
\text { sadness } \\
\text { anger }\end{array}$ & $\begin{array}{l}\text { contentment } \\
\text { love }\end{array}$ & $\begin{array}{l}\text { forced } \\
\text { judgment } \\
\text { with } 6 \text { emot- } \\
\text { lon labels }\end{array}$ \\
\hline $\begin{array}{l}\text { Fenster \& } \\
\text { Goldsteın, } \\
1971 \\
\text { Fenster, } \\
\text { Blake, \& } \\
\text { Goldstein, } \\
1977\end{array}$ & $\begin{array}{l}30 \mathrm{~m} \text { Am. adults, } \\
30 \mathrm{~m} \text { Am. child- } \\
\text { ren age } 10-12\end{array}$ & $\begin{array}{l}\text { 30m Am. adults } \\
\text { 30m Am. children } \\
\text { age } 7-9 \\
\text { 30m Am. children } \\
\text { age } 10-12\end{array}$ & $\begin{array}{l}\text { standard } \\
\text { sentence }\end{array}$ & 60 & $\begin{array}{l}\text { joy } \\
\text { fear } \\
\text { sadness } \\
\text { anger }\end{array}$ & $\begin{array}{l}\text { contentment } \\
\text { love }\end{array}$ & $\begin{array}{l}\text { forced } \\
\text { Judgment } \\
\text { with } 6 \text { emot- } \\
\text { Ion labels }\end{array}$ \\
\hline $\begin{array}{l}\text { Mattland, } \\
1978\end{array}$ & If Am. adult & $\begin{array}{l}\text { groups of } 32 \mathrm{~m} \\
\text { learning disabled, } \\
\text { emotionally dis- } \\
\text { turbed, and normal } \\
\text { Am. children age } 7-11 \\
\text { and } 12-16\end{array}$ & ? & $?$ & $\begin{array}{l}\text { joy } \\
\text { sadness } \\
\text { anger }\end{array}$ & $\begin{array}{l}\text { boredom } \\
\text { excitement } \\
\text { neutral }\end{array}$ & $?$ \\
\hline
\end{tabular}


Table 7 I (continued)

\begin{tabular}{|c|c|c|c|c|c|c|c|}
\hline Study & Encoders & Decoders & Speech sample & $\begin{array}{l}\text { Number of } \\
\text { stimuli }\end{array}$ & Emotions $^{a}$ & Emotions $^{b}$ & Task \\
\hline $\begin{array}{l}\text { Fechner, } \\
1978\end{array}$ & $\begin{array}{l}4 \mathrm{~m} \mathrm{Am}, 4 \mathrm{f} \\
\text { Am adults }\end{array}$ & $\begin{array}{l}6 \mathrm{~m} \text { Am and } 6 \mathrm{f} \\
\text { Am children age } \\
21 / 2 \text { to } 7\end{array}$ & $\begin{array}{l}\text { lowpass } \\
\text { filtered }\end{array}$ & 8 & $\begin{array}{l}\text { joy } \\
\text { fear } \\
\text { sadness } \\
\text { anger }\end{array}$ & & $\begin{array}{l}\text { forced judg- } \\
\text { ment with } 4 \\
\text { emotion } \\
\text { labels }\end{array}$ \\
\hline $\begin{array}{l}\text { McCluskey, } \\
\text { Niemı, \& } \\
\text { Alblas, } \\
1978\end{array}$ & $\begin{array}{l}6 \mathrm{~m} \text { Mexican } \\
\text { normal child- } \\
\text { ren age } 12 \text {, } \\
6 \mathrm{~m} \text { Mexican } \\
\text { disturbed } \\
\text { children age } \\
12\end{array}$ & $\begin{array}{l}20 \mathrm{~m} \text { Mexican normal } \\
\text { children age } 12, \\
20 \mathrm{~m} \text { Mexican dis- } \\
\text { turbed children } \\
\text { age } 12\end{array}$ & $\begin{array}{l}\text { lowpass } \\
\text { filtered }\end{array}$ & 48 & $\begin{array}{l}\text { joy } \\
\text { sadness } \\
\text { anger }\end{array}$ & love & $\begin{array}{l}\text { forced judg- } \\
\text { ment with } 4 \\
\text { emotion } \\
\text { labels }\end{array}$ \\
\hline $\begin{array}{l}\text { Blanck, } \\
\text { Rosenthal, } \\
\text { Snodgrass, } \\
\text { DePaulo, } \\
\text { Zuckerman, } \\
1981\end{array}$ & If Am & $\begin{array}{l}\text { a) } 121 \mathrm{~m} \text { and } 129 \mathrm{f} \\
\text { Am children age } 9 \\
\text { to } 15 \text {, } \\
46 \mathrm{~m} \text { and } 63 \mathrm{f} \text { Am } \\
\text { high school stud, } \\
32 \mathrm{~m} \text { and } 49 \mathrm{f} \mathrm{Am} \\
\text { college stud } \\
\text { b) } 24 \mathrm{~m} \text { and } 24 \mathrm{f} \mathrm{Am} \\
\text { children age } 11 \text { to } \\
14\end{array}$ & $\begin{array}{l}\text { two versions } \\
\text { lowpass } \\
\text { filtered and } \\
\text { random spliced }\end{array}$ & 40 & & $\begin{array}{l}\text { positive- } \\
\text { dominant, } \\
\text { negative- } \\
\text { dominant, } \\
\text { positive- } \\
\text { submissive, } \\
\text { negative- } \\
\text { submissive }\end{array}$ & $\begin{array}{l}\text { forced judg- } \\
\text { ment with } 2 \\
\text { situational } \\
\text { labels }\end{array}$ \\
\hline
\end{tabular}

a emotions also present in our study (joy $=$ happiness, contempt $=$ scorn)

b emotions not present in our study

$\mathrm{m}=$ male

$f=$ female

Am $=$ American Englısh

stud $=$ students 
The fourth column reveals that the speech material used as the carrier for emotional expressions has been quite varied: Both standard material, either meaningless or meaningful, and material of which the verbal content was not controlled have been employed. In the latter case, the verbal meaning was later removed by means of content masking techniques, either in the form of lowpass filtering or in the form of random splicing (for an explanation of these techniques, see Section 2.1). We think that the outcomes of studies in which these kinds of manipulated speech were used, should be interpreted with caution, in the first place because of the possibility that together with the verbal content non-verbal information relevant to the recognition of emotional meaning may have gotten lost, in the second place because it is not clear to what extent the subjects - and this may be particularly important in connection with the performance of very young children - have been confounded by the strange and uncommon sound of the manipulated stimuli.

From the fifth column it appears that there is a wide range in the number of stimuli offered in the various studies. In some studies, for example the ones reported on by Fenster an Goldstein (1971), and Fenster, Blake, and Goldstein (1977), the relatively short concentration span of young children does not seem to have been täken sufficiently into consideration.

The sixth and seventh columns show that there has been a small but stable set of emotions of which the recognizability has been examined: Joy and anger have been included in all but one study, and sadness in all but two. Fear and love have been considered in several studies as well. The choice of the other (emotional or attitudinal) categories investigated, however, makes a somewhat haphazard impression. It may also be seen that in most studies no more than six different categories have been offered, the only exception being the study by Gates (1927).

From the eighth and last column in Table 7.1 it appears that in all the studies reporting how the subjects were asked to respond a forced judgment task was used, i.e., a task in which the subjects were given a fixed set of alternatives from which to select the one best fitting the emotional portrayal presented. In most cases the alternatives consisted of emotion labels, such as "happy", "sad", etc. One reason for offering the subjects a fixed set of labels instead of asking them to describe the stimuli in their own words has to do with the limited verbal repertoire of children in comparison to that of adults (in Section 6.1 another reason for preferring the forced judgment task over the free choice task is given, a reason which is independent of the age of the subjects). However, with very young children even the choice among emotion labels might be problematic; that is why these labels have sometimes been replaced by pictorial representations of emotions.

The two main issues investigated in the studies included in Table 7.1 are the relative recognizability of negative (= unpleasant) and positive (= pleasant) emotions for children, and the developmental recognition of emotions as a 
function of age. The former issue is not considered in the present study, and will therefore not be dealt with. The interested reader is referred to the relevant studies (Dimitrovsky, 1964; Fenster, 1967; Fenster \& Goldstein, 1971; Fenster, Blake, \& Goldstein, 1977; McCluskey, Niemi, \& Albas, 1978). As for the latter issue, it appears that, at least between age 5 and 10 , there is a gradual and steady increase in the number of correct identifications with age (Dimitrovsky, 1964; Fechner, 1978; Fenster, 1967; Fenster et al., 1977; Gates, 1927). After age 10, at least for male subjects, the increase usually levels off. After age 20, in any case, no further increase in the number of correct responses takes place. On the contrary, both Turner (1964), using male subjects between the ages of 20 and 53, and Levy (1964), using male and female subjects between the ages of 21 and 51, found a significant although not very high negative correlation between age and the ability to correctly perceive emotional stimuli ( $r=-.45$ and $r=-.34$, respectively).

Very little is known about which emotions children tend to confuse with one another. Dimitrovsky (1964) is the only study reporting on confusions; she found that the confusions that her subjects made can be explained in terms of similarity in level of activity. Thus, the active emotions of anger and happiness tended to be confused, as did the passive emotions of sadness and love. A similar finding has been reported with respect to the performance of adults (Albas, McCluskey, \& Albas, 1976). It is thus suggested that both children and adults might employ the same dimensions in their categorization of vocal expressions of emotion. In view of the paucity of the available data, however, especially of data pertaining to confusions made by children, this hypothesis is strongly in need of further testing.

The question as to whether there is a difference in the performance of boys and girls has often been avoided by limiting the subjects to boys only. Those studies in which subjects of both sexes were included revealed a significant female advantage in the decoding of vocally expressed emotions, at least between age 6 and 15 (Dimitrovsky, 1964; Fechner, 1978). A female superiority was also found by Blanck, Rosenthal, Snodgrass, DePaulo, and Zuckerman (1981), who tested the ability of pre-high school and high school children at decoding lowpass filtered and random spliced speech samples. The speech samples had been derived from a film in which a female acts in twenty different emotional situations categorized with reference to four different types of emotion, each created by the crossing of two affective dimensions, namely positivity/negativity and dominance/submission.

From the above survey of the literature it appears that

(a) almost all developmental studies pertain to the recognition of American English expressions by American English children,

(b) in most studies a limited set of emotional categories has been offered,

(c) in all studies the response categories consisted either of emotion labels 
or of pictorial representations of emotions; in no study were the two types of response categories compared,

(d) no systematic comparison has been made between the specific confusions among emotions made by children and those made by adults, and

(e) little is known about the relative performance of boys and girls.

In the present chapter the recognition of vocal expressions of nine different emotions by Dutch children and a group of Dutch adults was examined. Specifically, the study aimed at answering the following questions:

(1) Can we replicate the finding that the number of correct identifications of vocal expressions of emotion increases with age?

(2) Is there a difference between girls and boys in the accuracy with which they respond to vocal expressions of emotion?

(3) Does the number of correct identifications depend on whether the subjects respond by choosing among labels or drawings?

(4) Are the confusions among emotions made by children similar to those made by adults?

\subsection{METHOD}

Recognition was assessed in two ways: by means of a forced judgment task in which the subjects responded to the stimuli by choosing among drawings ("drawing test") and by means of a forced judgment task in which the subjects responded by choosing among emotion labels ("label test").

\subsubsection{Subjects}

Fifty subjects, all native speakers of Dutch, participated in the research. There were five age groups, each consisting of five males and five females. The kindergarten which the youngest subjects attended and the primary school which the second, third, and fourth group attended, were located in Wijchen, a suburb of Nijmegen. The subjects in the oldest, fifth group were students from the University of Nijmegen. In Table 7.2 the mean age and the

Table 7.2 Mean age and range for five age groups of subjects

\begin{tabular}{llcc}
\hline Age group & Level & Mean age & Range $^{\mathrm{a}}$ \\
\hline 1 & Kindergarten & 5.8 & $56-59$ \\
2 & Second form & 78 & $76-79$ \\
3 & Fourth form & 97 & $96-910$ \\
4 & Sixth form & 117 & $113-11.9$ \\
5 & College students & 224 & $198-261$ \\
\hline
\end{tabular}

age is indicated in years and months 
ages of the youngest and oldest subject are given for each of the five age groups.

All five age groups participated in the drawing test; the label test was administered to groups 3,4 , and 5 only

\subsection{Speech material}

In both experıments use was made of eighteen tape-recorded realizations of the phrase daar is ie weer in which the nine emotions of disgust, surprise, shame, interest, joy, fear, contempt, sadness, and anger were each expressed twice, once by a female speaker and once by a male speaker. These eighteen expressions were a subset of the material which has been used in the recognition experiment with Dutch adults described in Chapter 6 (For more information on the stımuli, see Chapter 2 ) Daar is ie weer was selected for use in the present experiment despite the fact that on the whole the Dutch adults recognized the emotions expressed with this phrase significantly less well than the emotions expressed with the other three phrases employed (twee maanden zwanger, ze is bij de luchtmacht, zo'n grote Amerikaanse auto) This was done because the content of this particular phrase is comprehensible even to very young children, and because of the fact that it is the only phrase which can be relatively easily fitted into different context situations (see Section 72 3) Those realizations of the various emotions - each emotion had been produced by four different women and four different men - were selected which had been correctly identified by the adults most often: All eighteen emotional expressions which were used in the present experiment had yielded a percentage of correct responses of more than 60 (with ten response categories to choose from), except for the female expressions of disgust and surprise, and the male and female expressions of shame and interest (see Table 7.3 for the exact percentages)

Table 73 Percentages of correct identification of the stımuli attained by Dutch adults

\begin{tabular}{lll}
\hline & Male expression & Female expression \\
\hline Disgust & 92 & 58 \\
Surprise & 75 & 58 \\
Shame & 44 & 50 \\
Interest & 52 & 40 \\
Joy & 83 & 94 \\
Fear & 98 & 90 \\
Contempt & 79 & 79 \\
Sadness & 79 & 71 \\
Anger & 98 & 94 \\
\hline
\end{tabular}




\subsubsection{Instruments}

In the drawing test the subjects' ability to identify the emotional expressions was tested by asking them to respond to the stimuli by choosing among drawings which were provided with a brief explanatory text. In this explanation the use of the labels employed in the label test (see Section 7.2.5) was avoided. Each drawing depicted a daily event to which an adult could be imagined to react by realizing the phrase daar is ie weer with one of the nine emotions examined. There were two sets of nine drawings, each related to one of the nine emotional categories expressed on the tape. The two sets were identical except for the fact that in the one the main figure was a female (corresponding with the female voices) while in the other it was a male (corresponding with the male voices). The drawings were designed to carry as much information as possible (through facial expression, posture, gestures, etc.) about the emotion represented. In Figure 7.1 the "female" set is given as an example, with underneath each drawing the English translation of the matching explanation.

In order to determine whether the drawings were a clear representation of the various emotions, they were offered to a group of ten students, evenly divided over both sexes, from the University of Nijmegen before using them in the present experiment. This group did not include any of the students participating in the present recognition experiment. For the drawing identification task the subjects received a booklet with on each page one of the drawings and accompanying explanations as given in Figure 7.1. For each drawing plus explanation they were asked to indicate which one of the three emotion labels, combined and ordered in the way described in section 7.2.4, fitted best. The students reached a level of correct identification of $100 \%{ }^{1}$

\subsubsection{Response categories}

Both the drawing and the label test were multiple choice tests with three alternatives (we thought it would be too difficult for young children to handle the complete set of possible alternatives). Each item consisted of one key (the right emotion) and two distractors. The choice of the distractors was based on the outcomes of a hierarchical cluster analysis performed on the symmetrized version of the full data matrix based on the expressions with four phrases, which resulted from the recognition experiment with Dutch adults; for the - identical - outcomes of a hierarchical cluster analysis performed on the symmetrized version of the data matrix for one phrase, see Figure 9.4). From this cluster analysis it appeared that the nine emotions which the adults' study and the present developmental study had in common could be subdivided into three groups of three "related" emotions each, one group comprised of disgust, contempt, and anger; one group comprised of shame, sadness, and fear; and one group comprised of surprise, interest, and joy. In view of the nature of the emotions contained in each group, one could 
(disgust)

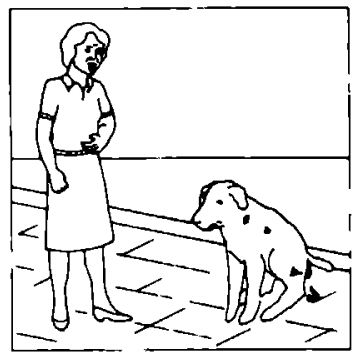

Mother thinks it very dirty of the filthy dog to shit on the sidewalk again

\section{(interest)}

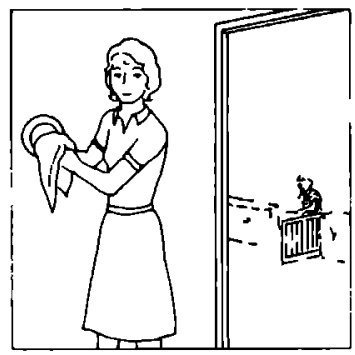

Mother would like to know whether Steve has returned (surprise)

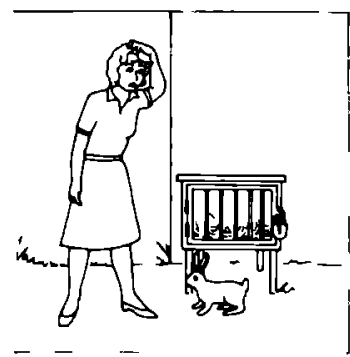

Mother doesn't understand how the rabbit managed to get out of its hutch again

(joy)

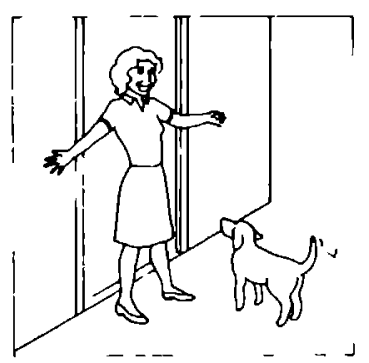

Mother is very glad that the dog has come back (shame)

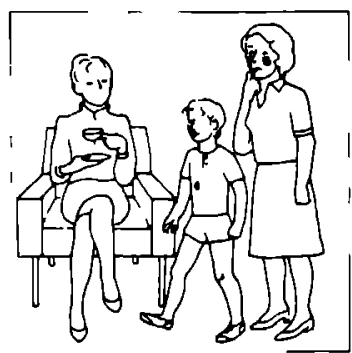

Mother really feels uncomfortable when Johnny comes in again with a snotty nose, especially since a classy aunt has called around

(fear)

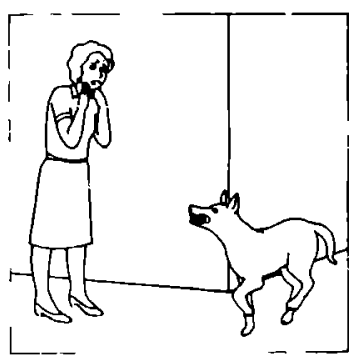

Mother finds the big mean dog very frightening (contempt)

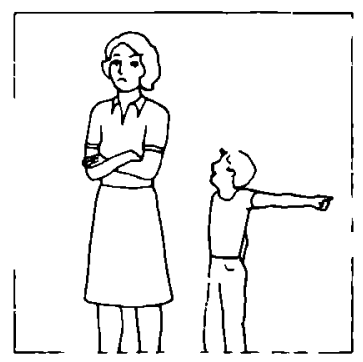

Mother thinks it is very low and mean of the boy from next-door to tell tales again (sadness)

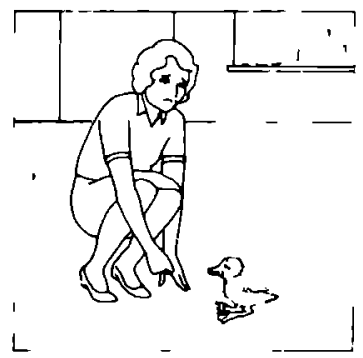

Mother feels very bad when she sees the sick duckling agaun (anger)

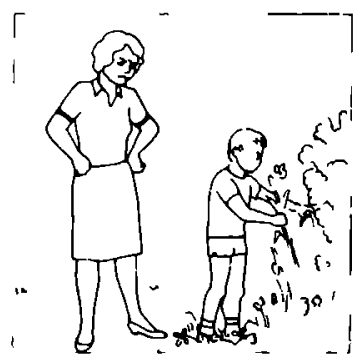

Mother is really mad at the boy for trampling her garden plants again 
say that the cluster analysis yielded a group of negative active emotions, a group of negative passive emotions, and a group of positive (or non-negative) emotions.

The distractors were selected in such a way that one was always an emotion related to the key, whereas the other one was not related to the key. Whenever the key was a positive emotion the non-related distractor was either a negative passive one $(2 X)$ or a negative active one $(1 X)$; whenever the key was a negative active emotion the non-related distractor was either a positive one $(2 X)$ or a negative passive one (1X); whenever the key was a negative passive emotion the non-related distractor was either a negative active one $(2 X)$ or a positive one (1X). Each single key had a fixed set of distractors, i.e., every time the key was, for example, disgust, whether in the drawing test or in the label test, the one distractor would be anger (an emotion related to disgust) and the other one surprise (an emotion unrelated to disgust). The order into which the alternatives were put was randomized, but again fixed for each single key throughout the two tests. (For more detailed information concerning the particular combinations and the order of the alternatives, see Appendix C.)

\subsubsection{Procedure}

In age groups $1,2,3$, and 4 the experiments took place in a quiet room of the school which the children attended. The tests were administered by two female experimenters to each child individually. The emotional expressions to be judged were played on a portable tape recorder (Uher 4200 Report). In age group 5 the experiments took place in a lecture room of the university. The tests were administered to the subjects as a group. For the presentation of the stimuli use was made of a tape recorder (Revox A77) and loudspeakers (Kef RR 104).

\section{Drawing test}

Each subject judged the same eighteen emotional expressions. The stimuli had been randomized, separately for the male and the female speakers. For half of the subjects in each of the age groups 1 to 4 the recordings of the female voices were played first and those of the male voices second; for the other half of the subjects in these groups the order was reversed. The subjects in age group 5 all started with the female voices.

The oral instructions, the style of which varied slightly for the different age groups, were about as follows:

We are very anxious to know how well you are able to tell from someone's voice how that person feels. You know that someone sounds completely different when he or she is angry than when he or she feels, for example, happy. You are going to hear different men and women saying the same thing, namely daar is ie weer, in different ways. We would like you to listen very carefully to the way in which these words are said. Each time you will see 
three drawings. We ask you to point to (age groups 1 to 4), mark (age group 5) the drawing which fits best the sentence you have heard.

The procedure was practiced by means of three test stimuli. When it was clear that the task was understood each subject received a booklet with as many pages as there were stimuli. On each page three drawings were presented, the male version with the male voices, and the female version with the female voices, combined and ordered in the way described in Section 7.2.4. With the subjects in the age groups 1 to 4 the explanations that went with the drawings were presented orally by one of the experimenters, while the other one operated the taperecorder and noted down which drawing the subject pointed at. With the subjects in age group 5 the explanations were presented in written form underneath the drawings that they accompanied. This group responded by marking themselves the drawing of their choice on an answer sheet. In all five age groups each stimulus was played four times before the next one was presented.

The test took about 20 minutes.

\section{Label test}

As an informal test had revealed that a number of emotion labels, such as shame, interest, and contempt, are generally not known before age 9, the label test was carried out in the three oldest groups only. The task was administered prior to the drawing test - this means that in age groups 3, 4, and 5 the instructions for the drawing test could be offered in an abbreviated form - and took about 10 minutes. The procedure followed and the instructions given were the same as in the drawing test - the subjects in age groups 3 and 4 took the test individually and the subjects in age group 5 as a group except for the fact that the response categories consisted of labels instead of drawings, and the fact that the subjects in age groups 3 and 4 instead of pointing at the right response category now marked the answers themselves.

The labels used were walging ("disgust"), verrast ("surprised"), schaamte ("shame"), belangstelling ("interest"), blij ("happy"), bang("afraid"), minachting ("contempt"), bedroefd ("sad"), and boos ("angry"). The nouns were combined with the verb voelen ("to feel") and the adjectives with the copula zijn ("to be"). Thus, the subjects would be asked to respond to a stimulus by marking one of the following three alternatives: "Does father feel contempt?" "Is father happy?" "Is father angry?"

After the task had been explained by means of three test stimuli, the tape recorder was switched on, and the subjects were left to themselves to complete the scoring of the eighteen experimental stimuli. Those subjects in age groups 3 and 4 which were to start with the female voices in the drawing test now heard the male voices first; for the other subjects in these groups the opposite was the case. All subjects in age group 5 started with the male voices. 


\subsection{RESULTS}

In order to answer the first two questions formulated in the introduction, i.e., whether or not there was an increasing number of correct identifications with age, and whether or not there was a difference between males and females in the accuracy with which they responded, two analyses of variance (repeated measurements design) were carried out, one for the drawing test and one for the label test, with three fixed factors, namely age ( 5 for the drawing and 3 for the label test), sex (2), and emotion (9). A factorial design was used with one observation per cell. The error term was the residual variance age $\times \operatorname{sex} \times$ emotion. Input to the program were the mean numbers of correct responses in each age group, separated for emotion and sex of subject.

\subsubsection{Factor age}

The results of the analyses of variance showed that for both the drawing test and the label test there was a significant overall effect for the factor age $(F(4$, $32)=10.46, p<.01$ and $F(2,16)=5.20, p<.05$, respectively). Inspection of the mean number of correct responses in the various age groups (Table 7.6) reveals that with chronological age there is a gradual, steady increase in the ability to identify emotional expressions. Thus, the first question may be answered positively. For both tests the increase up to age 11 is relatively smooth. For the label test the increase after age 11 is negligible. For the drawing test the curve continues to rise, but only due to the performance of the males; the females have stopped making any progress.

In order to assess which age groups differed significantly from each other Tukey's HSD test, an a posteriori comparison test (Kirk, 1968, p. 88 - 90), was carried out on the cell means. We adopted a level of significance of $5 \%$. It appeared that for the drawing test the differences between age group 2 and age group 5, between age groups $I$ and 4 , and between age groups 1 and 5 were significant. For the label test there was a significant difference between age group 3 and age group 5 .

Inspection of the number of correct identifications in the different age groups per emotion shows that for the drawing test the increase with age is mainly due to the growing ability to identify expressions of surprise, shame, interest, and contempt (Table 7.4). A binomial estimate of probability, the outcomes of which are indicated with an asterisk in the table, revealed that surprise, for example, was only identified beyond chance in age group 5 , and shame only in age groups 4 and 5 .

Nevertheless, it may be seen from the bottom row in Table 7.4 that the number of correct scores averaged over emotions is significant for all age groups. That, on the whole, even the five-year-olds did better than chance is largely due to the fact that with respect to the recognition of joy, fear, sadness, and anger the youngest subjects did almost or just as well as the older ones. In fact, across all age groups hardly any mistakes were made in the identification of these four emotional categories. The data thus suggest clear effects of the factor emotion and the interaction between emotions and 
Table 74 Recognition by means of drawings: Number of correct identufications of nine emotions in five age groups

\begin{tabular}{lccccc}
\hline & Age group & & & \\
\cline { 2 - 6 } & 1 & 2 & 3 & 4 & 5 \\
\hline Disgust & $15^{* * *}$ & 9 & $14^{* * *}$ & $16^{* * *}$ & $17^{* * *}$ \\
Surprise & 1 & 7 & 4 & 9 & $10^{*}$ \\
Shame & 3 & 2 & 2 & $11^{* *}$ & $11^{* *}$ \\
Interest & 9 & $10^{*}$ & $16^{* * *}$ & $13^{* *}$ & $14^{* * *}$ \\
Joy & $17^{* * *}$ & $20^{* * *}$ & $20^{* * *}$ & $20^{* * *}$ & $20^{* * *}$ \\
Fear & $16^{* * *}$ & $20^{* * *}$ & $17^{* * *}$ & $18^{* * *}$ & $18^{* * *}$ \\
Contempt & 4 & $10^{*}$ & $15^{* * *}$ & $14^{* * *}$ & $18^{* * *}$ \\
Sadness & $18^{* * *}$ & $20^{* * *}$ & $17^{* * *}$ & $19^{* * *}$ & $19^{* * *}$ \\
Anger & $19^{* * *}$ & $15^{* * *}$ & $20^{* * *}$ & $15^{* * *}$ & $18^{* * *}$ \\
Mean & $11.3^{* *}$ & $12.6^{* *}$ & $13.9 * * *$ & $150^{* * *}$ & $16.2^{* * * *}$ \\
\hline
\end{tabular}

Note. Maxımum score $=20$

- $p<05$

$* p<.01$

$\cdots * p<.001$

age. This was confirmed by the outcomes of the analysis of variance $(F(8,32)$ $=42.26, p<.01$ and $F(32,32)=2.49, p<.01$, respectively).

The data in Table 7.4 finally reveal that although the curve of the mean number of correct scores in the drawing test continues to rise after the age of 11 , this is due to the increase in correct responses for a very few specific emotions, especially contempt and anger. And for these emotions the in-

Table 75 Recognition by means of labels: Number of correct identilications of nine emotions in three age groups

\begin{tabular}{llll}
\hline & Age group & & \\
\cline { 2 - 4 } & 3 & 4 & 5 \\
\hline Disgust & $15^{* * *}$ & $18^{* * *}$ & $19^{* * *}$ \\
Surprise & $11^{* *}$ & $16^{* * *}$ & $16^{* * *}$ \\
Shame & 9 & $14^{* * *}$ & $12^{* *}$ \\
Interest & 6 & $13^{* *}$ & $13^{* * *}$ \\
Joy & $14^{* * *}$ & $15^{* * *}$ & $19^{* * *}$ \\
Fear & $12^{* *}$ & $18^{* * *}$ & $18^{* * *}$ \\
Contempt & $15^{* * *}$ & $11^{* * *}$ & $15^{* * *}$ \\
Sadness & $20^{* * *}$ & $19^{* * *}$ & $18^{* * *}$ \\
Anger & $20^{* * *}$ & $19^{* * *}$ & $16^{* * *}$ \\
Mean & $13.7^{* * *}$ & $159^{* * *}$ & $16.2^{* * *}$ \\
\hline
\end{tabular}

Note. Maximum score $=20$

- $p<05$

$* \quad p<.01$

$* * * p<.001$ 
crease from age group 4 to age group 5 is mainly caused by the fact that age group 4 did worse than age group 3.

The results for the label test offer a similar although not completely identical picture (Table 7.5) While the scores for disgust, contempt, sadness, and anger are roughly comparable for both tests, the expressions of surprise and shame yielded a considerably higher number of correct identifications in the label test than in the drawing test, for the expressions of interest, joy, and fear the opposite was the case, especially in age group 3.

\subsubsection{Factor sex of subject}

The factor sex of subject did not have a significant effect on the scores, neither in the drawing test nor in the label test $(F(1,32)=3.47, p>.05$ and $F$ $(1,16)=0.18, p>.05$, respectively) This means that from a statistical point of view the second question should be answered in the negative, 1.e., there is no difference between males and females in the accuracy with which they responded to the stimuli. Inspection of the mean scores separated for the two sexes (Table 76 ), however, shows that while for the label test the scores for males and females do indeed hardly differ, for the drawing test there is a slight but consistent difference in favour of the females. The female super1ority is most notable for the children; in the adult group it has been reduced to almost nothing. In order to make sure that the absence of a significant sex effect for the drawing test was not due to the inclusion of the scores of the group of adults, an analyıs of vanance was carned out on the scores of age groups $1,2,3$, and 4 only. The factor sex of subject still farled to reach significance $(F(1,24)=384, p>.05)$.

\subsection{Factor test}

A third analysis of variance, with the fixed factors of age (3), test (2), and emotion (9), was carried out in order to determine whether the number of correct identifications depended on whether the subjects responded by

Table 76 Mean number of correct identifications in different age groups, males and females separately and combined, for two tests

\begin{tabular}{|c|c|c|c|c|c|c|}
\hline \multirow[t]{2}{*}{ Age group } & \multicolumn{3}{|c|}{ Drawing test } & \multicolumn{3}{|c|}{ Label test } \\
\hline & Males & Females & Males +Females & Males & Females & Males+Females \\
\hline 1 & 98 & 106 & 102 & - & - & - \\
\hline 2 & 108 & 118 & 113 & - & - & - \\
\hline 3 & 122 & 128 & 125 & 124 & 122 & 123 \\
\hline 4 & 126 & 144 & 135 & 146 & 140 & 143 \\
\hline 5 & 144 & 146 & 145 & 146 & 146 & 146 \\
\hline
\end{tabular}

Note Maxımum score $=18$ 
choosing among drawings or labels A factorial design was used with one observation per cell. The error term was the residual variance age $X$ test $X$ emotion. Input to the program were the mean number of correct scores for each emotion in the drawing and in the label test, separately for each of the three oldest age groups. The factor test was not significant $(F(1,16)=001$, $p>.05$ ). This means that the third question must be answered in the negative. This is true, of course, for age groups 3,4 , and 5 only. The relative performance of the younger subjects could not be compared, since the label test had not been admınıstered in age groups 1 and 2 . It should be noted, moreover, that the negative answer to the third question is only a global one, as appears from the significant interaction between test and emotion $(F(8,16)=466$, $p<01$ ); we had already observed that some emotions were identified correctly more often in one test than in the other.

\subsubsection{Confusions}

A chi square test was used to answer the fourth question, i.e., whether or not the confusions made by the children were similar to the ones made by adults. The chi squares were computed separately for the two tests and the various age groups, by subtractıng the number of correct responses, summed over all subjects and stımul, from the total number of responses, and by comparing the spread of the incorrect scores over the two categories of distractors (one of emotions related to the key (=confusions within clusters) and one of emotions not related to the key (= confusions between clusters)) with the spread based on chance expectancy In all cases Yates' correction for cont1nuity was applied (Ferguson, 1976, p. 201-202). The outcomes are presented in Tale 7.7.

Table 77 Number of confusions within and between clusters of emotions for various age groups in two tests

\begin{tabular}{llll}
\hline Age group & Within & Between & Chi square \\
\hline & & Drawing test & \\
\hline 1 & 56 & 22 & $1396^{*}$ \\
3 & 52 & 15 & $1934^{*}$ \\
4 & 48 & 5 & $3328^{*}$ \\
5 & 38 & 7 & $2000^{*}$ \\
\hline & 30 & 5 & $1786^{*}$ \\
\hline 3 & & Label test & \\
4 & 48 & 9 & $2533^{*}$ \\
5 & 28 & 9 & $86^{*}$ \\
\hline
\end{tabular}

$d f=1$ for each group

$p<01$ 
All eight chi squares computed were significant at the $1 \%$ level. Thus, the fourth question has to be answered in the affirmative: For both tests the confusions made by the children were similar to the ones made by adults, both when the performance of the children (age groups 1 to 4) is compared with the performance of the adults (age group 5) in the present study, and when the performance of the children is compared with the performance of the adults in the recognition experiment on which the distinction between the two kinds of distractors was based. Both children and adults confused positive emotions far more often with one another than with active negative or passive negative emotions; active negative emotions were confused with one another far more often than with passive negative or positive emotions; and passive negative emotions were confused with one another far more often than with active negative emotions or positive emotions.

Inspection of the confusions further revealed that, for the drawing test, of the total of forty-nine confusions which were made in age groups 1,2,3, and 4 among emotions not belonging to the same cluster twenty-two concerned a confusion between a positive and an active negative emotion, seventeen a confusion between an active negative and a passive negative emotion, and ten a confusion between a passive negative and a positive emotion. A chi square showed that the distribution of these confusions did not differ significantly from chance $\left(\chi^{2}(2)=3.72, p>.10\right)$. The summed data for the label test were 10,7 , and 1 , respectively. For this distribution chi square was not significant either $\left(\chi^{2}(2)=5.45, p>.05\right)$. For the combined data of the drawing test and the label test, however, chi square did prove to be significant $\left(\chi^{2}(2)=9.09, p<.02\right)$. Since all positive emotions included in the present study may be considered to be active rather than passive, this means that there is a tendency for confusions by children to be characterized by similarity in level of activity rather than by similarity in evaluative meaning. It may further be noted that confusions among (active) positive emotions and passive negative emotions occurred by far the least often.

\subsection{DISCUSSION}

\subsubsection{Factor age}

The results of the present study showed a gradual but steady increase in the mean number of correct identifications with age, both when the subjects responded by choosing among drawings and when they responded by choosing among labels. The finding of a growing sensitivity to vocal expressions of emotion as a function of age is consistent with the results of all developmental research into emotions in which this question has been considered to date (Dimitrovsky, 1964; Fenster, 1967; Fenster, Blake,\& Goldstein, 1977; Gates, 1927; Maitland, 1978; McCluskey et al., 1975; Scherer \& Oshinsky, 1977). This general finding also parallels what is known about the development of 
sensitivity to facial expressions of emotion (Dickey \& Knower, 1941; Gates, 1923: Gitter, Mostofsky, \& Quincy, 1971; Odom \& Lemond. 1972: Maitland. 1977; Wiggers, 1977).

The fact that the mean number of correct identifications in the group of the five-year-olds already exceeded chance, a finding also reported by Dimitrovsky (1964), suggests that the recognition of vocally expressed emotions starts at a very early age (see Chapter 8 ). Our findings are not conclusive about whether or not the sensitivity after age 11 further increases, since it did so for the males but not for the females, at least in the drawing test. The label test showed virtually no further progress after this age at all.

However, the data with respect to the average performance in each age group are not very informative and possibly even misleading, the increase in the mean number of correct responses being due for a large part to a growing sensitivity to a few specific emotions, particularly surprise, shame, interest, and - in the drawing test - contempt. With respect to the expressions of disgust, joy, fear, sadness, and anger the five-year-olds did almost just as well as the adults. In fact, it appears that it is largely the stimuli that had been relatively hard for the group of adults to identify (see Table 7.3) that made it possible in the present experiment for the age effect to show up. It could be that after age 11 the increase in the number of correct responses would have continued, or, in other words, that there would have been less of a ceiling effect, if the realizations of the other emotions had been more ambiguous too, or if more difficult emotional categories had been selected for study. Of course, in that case there would have been the risk that the youngest subjects would not have been able to demonstrate their abilities.

It is probably very difficult to develop a test which can discriminate over the complete age range from early childhood to adulthood. Perhaps a solution should be sought not so much in adapting the stimuli but rather in adapting the kind and number of alternatives among which to choose. In the present study the rationale behind opting for a three choice test was based on the - limited - cognitive capacities of the youngest subjects. And inspection of the number of "between" confusions (see Table 7.7) reveals that indeed for the five- and seven-year-olds the test has functioned as such. The older subjects, however, hardly ever responded by choosing an emotion which was not related to the key; for them the test may have functioned as a two choice test or as a two-step two choice test in which the chance of success in the first step was much greater than $50 \%$. In fact, the data in Table 7.7 suggest that much of the growing sensitivity of the younger subjects may be traced back to learning to identify the unrelated distractor as an unlikely label for the stimulus offered. Once this capacity has been acquired - in our experiment somewhere between the seventh and ninth year of age - discrimination is further refined to distinguish between the related alternatives. More insight into this process could be gained by conducting parallel experiments in which the same stimuli were offered with differing numbers and combinations of response categories. 
It is difficult to compare our findings with those obtained in previous developmental research into vocal expressions of emotion, firstly because the emotional categories examined in those studies coincide only partially with the ones examined in the present study, and secondly because only in a few cases data with respect to individual emotions have been reported. It appears, in any case, that in the study by Fenster et al. (1977), in which a significant age effect was found in a combined analysis of variance, the increase in the mean number of correct identifications was mainly due to a growing sensitivity to a few specific emotions as well. Of the separate analyses of variance performed on the scores of each of the six emotions studied (happiness, contentment, love, fear, sadness, and anger) only two revealed a significant age effect, namely fear and sadness. For the other four emotions the performance of the youngest age group (age 7-9) was comparable to that of the adults. With regard to the emotions of anger and happiness these outcomes are consistent with ours. But then, the findings for fear and sadness are not.

Dimitrovsky (1964), who studied happiness, love, sadness, and anger, also found some evidence of interaction between age and emotion. However, in her study a possible ceiling effect, which was most obvious for happiness, sadness, and anger, was only apparent from age 9 on. The fact that for these emotions in the present study a ceiling effect was found at an earlier age may have to do with the extensive selection procedure to which our speakers and stimuli had been submitted (see also Sections 2.2.1 and 2.2.4). In Dimitrovsky's study there had been a selection too, but it is not clear according to which criteria. In the study by Fenster et al. (1977) the stimuli had not been edited. Moreover, in the latter experiment the speakers had been asked to express a mild degree of the respective emotions. Thus, it could be that the expressions in the present experiment were more extreme and less ambiguous than those in the studies by Dimitrovsky (1964) and Fenster et al. (1977), thus making it easier for all age groups to identify the emotions correctly.

It is interesting to note that the relative recognizability of the various emotions as appearing in the present study is in accord with the results of developmental studies on the recognition of facially expressed emotions. In those studies it was also found that joy, fear, sadness, and anger are recognized at an earlier age than, for example, shame and contempt (Gates, 1923; Izard, 1971; Wiggers, 1977).

\subsubsection{Factor sex of subject}

In the present study no significant effect was found of the factor sex of subject, neither in the drawing test nor in the label test. This finding is inconsistent with findings reported earlier of a significant female advantage in the decoding of vocal expressions of emotion during childhood (Dimitrovsky, 1964; Fechner, 1978). From this it follows that our findings do not confirm certain hypotheses which have been put forward to explain the 
supposedly greater sensitivity of girls, such as the hypothesis formulated by Dimitrovsky (1964) and the one formulated by Blanck et al. (1981).

Dimitrovsky (1964) speculates that "one might expect preschool boys and girls to show little or no difference in a variable such as emotional sensitivity, but gradually with age, as the cultural expectations that a girl is sensitive and intuitive and a boy is active and 'objective' play an increasingly important role in development, one would expect greater differences in the degree to which boys and girls attend to emotional stimuli and thus acquire skill in discriminating emotional expressions" (p. 84). Dimitrovsky bases her ideas on the fact that in her experiment the five-year-olds were the only group in which the boys did better than the girls, and the fact that after age 10 the curve for the girls continued to rise while that for the boys did not.

Blanck et al. (1981) formulated a different hypothesis. They claim that with regard to the variable sex of subject a distinction should be made between different channels of communication. They therefore examined the recognition of four types of nonverbal cues (face, body, voice, mixed), arranged from the most controlable channel to the least controllable (most "leaky") one, in three age groups, namely pre-high school students, high school students, and college students. The outcomes confirmed their hypothesis that as age increases females lose more and more of their advantage for the more leaky channels (voice and mixed), while gaining more and more of their advantage for the less leaky channels (face and body). Blanck et al. believe that as women grow older they politely refrain from decoding effectively the less controllable cues of emotional communication because they learn through experience that it damages social relationships if one is too skillful at decoding nonverbal messages that one is not intended to receive. Blanck et al. thus agree with Dimitrovsky that during childhood girls will do better than boys; unlike Dimitrovsky, however, they predict a decrease in the ability of females to decode vocal expressions of emotion with age.

Since in our study no significant difference in favour of the girls was found, neither of the two above mentioned hypotheses is confirmed. Moreover, we find both hypotheses very vague and speculative. We have the impression that one is sometimes too quick to use sex role standards, which themselves are still in need of further investigation, as a panacea for the explanation of results which otherwise might be difficult to interpret.

\subsubsection{Factor test}

Our study did not reveal a significant overall difference between the performance of age groups 3,4, and 5 on the drawing test and their performance on the label test. This outcome suggests that, generally speaking, for these age groups the emotion labels had a clear meaning and that the concrete situatio$\mathrm{n}$-al cues contained in the drawings did not provide any extra information.

There are several reasons to assume that a different outcome would have been obtained if the performance of the younger subjects had been compar- 
ed. In the first place, an informal test had revealed that certain emotion labels, such as shame, interest, and contempt - labels for which a simpler synonym is hard to find - were generally not known to the five- and sevenyear-olds. It may be confidently assumed that this deficit would have negatively affected their performance on the label test, had it been administered to them. In the second place, there have been a number of developmental studies on the recognition of facially expressed emotions which have shown that younger children do better with the drawing method than with the label method (Dashiell, 1927; Wiggers, 1977). Interestingly enough, Wiggers' finding that up to age 8 his subjects did better with the drawing method whereas the nine-year-olds did better with the label method, suggests that age 9 is indeed crucial in this respect, and that from that age on, at least in a formal recognition task, situational information is redundant.

On the other hand, although there was no overall difference between the performance on the drawing test and the label test, the significant interaction between test and emotion revealed that some emotions, particularly interest, joy, and fear in age group 3, yielded a higher score with the drawing method whereas other emotions, particularly surprise and shame, yielded a higher score with the label method. The former outcome suggests that, at least for the three emotions in question, the nine-year-olds still inferred a more complete picture of which emotion was represented from the drawings than from the labels. The latter outcome seems harder to explain since in the drawing identification task (see Section 7.2.3) administered to adults the drawings had been matched correctly with the labels $100 \%$ of the time. It thus does not seem very likely that it was unclear to the subjects which emotions the drawings represented.

Perhaps some remarks made by Brown (1980) are relevant in this context. Brown argues that a number of recognition studies did not really measure judgment accuracy, especially those in which the speakers were offered a context of situation (text or scenario) - a context of situation designed by the experimenter - in which to produce a certain emotion, while the listeners were asked to attach emotion labels to the utterances thus produced. He states that "in the strictest terms, a true accuracy study would allow judges to read the scenarios and then judge which utterance is the result of portraying which scenario, since the ultimate definition of what emotion each actor is portraying is in the content of the scenario, not in the emotion label applied to it by the experimenter" (p. 240).

Following the same line of reasoning in the present experiment, the label test measured true judgment accuracy since both speaker and listener proceeded on the basis of emotion labels. For the drawing test, however, true judgment accuracy would only have been assessed if the utterances offered had been realized with a view to matching the drawings. As it is now, speaker and listener may have had rather diverging ideas in mind while carrying out their task, since the former was offered an emotion label which he or she was free to interpret the way he or she wanted whereas the interpretation of the 
latter was determined and probably restricted by the context of situation represented by the drawings, a context which had been designed to fit the particular meaning of the phrase daar is ie weer. There is, in other words, a good chance that for some emotional categories the meaning expressed in the utterances did not completely match the meaning expressed by the drawings. However, why this would have affected the expressions of surprise and shame more than those of the other emotions remains unclear.

The above considerations indicate that in order for the results of developmental recognition experiments to be valid - and this is especially relevant to studies in which very young children serve as subjects - careful attention should be given to the construction of the response categories. It seems that in research into vocally expressed emotions one has a choice between three types of response categories: emotion labels, stylized drawings or pictures of facial expressions, and situational representations. As for emotion labels, it has been reported (Amen, 1941, cited in Gilbert, 1969) that "simple" labels, such as "happy", "scared", "sad", and "mad", already occur in children's vocabulary at age 4 . With respect to other labels we know of no systematic observations. If any of these more "complex" labels are used, it seems wise, therefore, to check the subjects' knowledge before the recognition experiment is conducted. It has to be admitted that with very young children who are not able to read or write this is in itself a difficult problem.

If, in one way or another, the subjects' knowledge of (some of) the emotion labels should prove to be insufficient, the researcher could opt to use stylized drawings or pictures of facial expressions. Again, the appropriateness of these would have to be tested independently. Apart from the practical problem that research into the recognition of facial expressions has convincingly shown that for a number of emotions it will be almost impossible to find unambiguous representations, we envisage the problem of deciding which testing method to use, the choice of emotion labels as response categories not being available at this point.

A third alternative is the use of situational representations, such as the ones used in the drawing test in the present experiment. If this alternative is chosen, it should be realized that the possibility of constructing appropriate situational response categories is heavily dependent upon the nature of the verbal content of the speech material used as the carrier for the emotional expressions. The more specific this emotional content, the more restrictions will operate on the concrete context of situation in which it may fit, and the greater the chance that the emotional meaning inferred from the context constructed is narrowed down to only one or a few emotional aspects, or biased towards a particular degree of emotional intensity. Morover, if the remarks made by Brown are to be taken into account, the speakers would have to produce their expressions with a view to matching the situational representations. Of course, then the results of the subsequent recognition experiment could no longer be interpreted as information about the accuracy of identification of broad emotional categories. 


\subsubsection{Confusions}

The outcomes of the present study finally revealed that the confusions made by children are similar to the ones made by adults: Both tend to make confusions within each of the three clusters distinguished, namely a positive one, comprising surprise, interest, and joy; an active negative one, comprising disgust, contempt, and anger; and a passive negative one, comprising shame, fear, and sadness. These findings suggest that the categorization of the stimuli may be accounted for by the functioning of at least two distinctions, namely passive vs. active and negative vs. positive.

For both tests, in those cases in which confusions among emotions not belonging to the same cluster are made, (active) positive and active negative emotions are confused with one another slightly more often than are passive negative and active negative emotions. This could perhaps be regarded as an indication that in the categorization similarity in level of activity is assigned a somewhat heavier weight than similarity in evaluative meaning. Our findings would then be consistent with the outcomes of the developmental study conducted by Dimitrovsky (1964), which revealed that the emotions of happiness and anger, and those of love and sadness, were confused more often with one another than happiness and love, and anger and sadness. However, in our experiment the numerical data in support of this interpretation are not very strong - not by far as strong as in Dimitrovsky's study -and relate to active emotions only, passive positive emotions not being included. The fact that (active) positive emotions and passive negative emotions were hardly ever confused suggests that in the categorization of the various emotions both distinctions have played an important, and fairly independent role.

\section{NOTE}

1. An optimal test of the clearness of the drawings plus explanations would have involved the use of children as subjects (this would only have been possible with older children, the meaning of a number of the emotion labels generally not being known before age 9). On the other hand, on the basıs of the $100 \%$ correct identification attanned by the adults, we think it may confidently be assumed that for children also the drawings plus explanations must have been fairly easy to interpret. 


\section{Recognition by toddlers}

\subsection{INTRODUCTION}

In the developmental recognition study reported on in Chapter 7, a binomial estimate of probability revealed that the emotions of disgust, joy, fear, sadness, and anger were identified by five-year-olds significantly $(p<.01)$ more often than chance (at least fourteen out of twenty times with three response categories to choose from). It further appeared that the recognition of the expressions of interest failed to reach significance at the $5 \%$ level by one score, and that the expressions of surprise, shame, and contempt yielded very low numbers of correct responses. These findings indicate that, by the age of five, with respect to the recognition of at least a number of emotional categories children must already have undergone a fair amount of development. Dimitrovsky (1964), who examined the developmental recognition of happiness, sadness, love, and anger, came to the same conclusion on the basis of her finding that the five-year-olds as a group did significantly better than chance.

Dimitrovsky's suggestion that the question of sensitivity to vocally expressed emotions before the age of five be investigated, was not taken up until 1978 when Fechner set up a study to investigate the development of the recognition of happiness, fear, sadness, and anger in three modalities, namely the face, gestures, and the voice. Among the subjects, twelve three-yearolds (six males and six females) and twelve $2 \frac{1}{2}$-year-olds (six males and six females) were included. As for the vocally exptessed emotions, each subject heard the lowpass filtered versions of four male and four female expressions of each of the four emotional categories investigated. The instructions used were, "Tell me if this person is happy, scared, sad, or mad".

The information provided on the outcomes of the study is scanty. Fechner reports that the $2 \frac{1}{2}$-year-old children did not understand the task, without specifying what exactly caused the problems. It could be that the labels among which the children had to choose were not clear; it could also be that the subjects were confounded by the sound of the filtered stimuli. With regard to the age at which the emotional expressions were correctly identified, Fechner states at one point that "the children recognized voice tones above chance at age 4" (p. 2568) and at another that "voice tones [were] not [recognized] till age 6" (ibid.). A few lines further it then appears that the girls 
recognized the vocal expressions of fear at age 3 , two years sooner than the boys. It is finally observed that the girls did significantly better than the boys.

However vague and even contradictory these statements may be, it seems justified to conclude that in Fechner's study at age $2 \frac{1}{2}$ and 3 most of the emotional expressions were not correctly identified, and that there was a gender-difference in favour of the girls. The latter finding agrees with the hypothesis put forward by Blanck et al. (1981); it does not fit in with the hypothesis put forward by Dimitrovsky (see Section 7.4.2).

In the present chapter a recognition study is presented in which a group of $31 / 2$-year-olds, evenly divided over both sexes, served as subjects; it differs from Fechner's study in that the stimuli were not manipulated, that the subjects had to respond by choosing among drawings instead of labels, and that six instead of four emotional categories were offered.

The questions which the present study tried to answer may be summarized as follows:

(1) Are $3 \frac{1}{2}$-year-old children able to identify vocal expressions of emotion beyond chance expectancy?

(2) Is there a difference between $3 \frac{1}{2}$-year-old girls and $3 \frac{1}{2}$-year-old boys in the accuracy with which they identify vocal expressions of emotion?

(3) Are the confusions made by $3 \frac{1}{2}$-year-old children similar to the ones made by adults?

\subsection{METHOD}

Recognition was assessed by means of a forced judgment task in which the subjects responded to the stimuli by choosing among drawings.

\subsubsection{Subjects}

Ten children, five girls and five boys, attending three different day-nurseries in and around Nijmegen, participated in the experiment. All were native speakers of Dutch. The mean age was 3.6 years, the youngest child was 3.3 years and the oldest one 3.8 years.

\subsubsection{Speech material}

Use was made of twelve tape-recorded realizations of the phrase daar is ie weer, with which the six emotions of disgust, interest, joy, fear, sadness, and anger were each expressed twice, once by a female speaker and once by a male speaker. These twelve expressions were a subset of the eighteen expressions which served as stimuli in the developmental study described in Chapter 7. (For the motivation behind the choice of the phrase and the eighteen stimuli, see Section 7.2.2; for general information on the stimuli, see Chapter 
2.) The number of stimuli was reduced from eighteen to twelve because of the shorter concentration span of the subjects used in the present study. The choice of the particular emotional categories to be omitted was based on the responses of the five-year-olds participating in the developmental study. In this group the number of correct identifications of surprise, shame, and contempt was so low that the presentation of the expressions of these emotions to even younger children did not seem to make much sense.

\subsubsection{Instruments}

The response categories consisted of two sets of drawings, each related to one of the six emotions studied. In one set the main figure was a male (corresponding with the male voices) and in the other one a female (corresponding with the female voices). The drawings were identical with the ones used in the drawing test of the developmental study reported on in Chapter 7, except, of course, for the fact that the emotions of surprise, shame, and contempt were not represented. The explanations that went with the drawings were extended and changed in order to match the terminology and style commonly adopted when speaking to three-year-olds. In Figure 8.1 the "male" version of the drawings is presented with underneath the English translation of the matching explanations.

\subsubsection{Response categories}

For each stimulus the subjects responded by choosing among three drawings plus explanations. These drawings were combined according to the method used in the developmental study (see Section 7.2.4). Thus, there would always be one drawing which was the key (the right emotion), one which represented an emotion related to the key, and one which represented an emotion not related to the key. Of course, since the number of emotional categories had been reduced from nine to six, the specific combinations were different. The other conditions, that each single key had a fixed set of distractors and that the order in which the alternatives were put was random but again fixed for each single key throughout the test, were maintained. In short, there were six combinations of alternatives (see Appendix D), which each occurred twice in the test, once for the female expressions and once for the male expressions.

\subsubsection{Procedure}

The test was administered by two female experimenters in a quiet room of the day-nursery which the subjects attended. Prior to the test all children present in the day-nursery were taken to the experimentation room where the functioning of the tape recorder (Uher 4200 Report) was demonstrated by playing some emotional expressions realized by the same speakers as used in 
(dısgust)

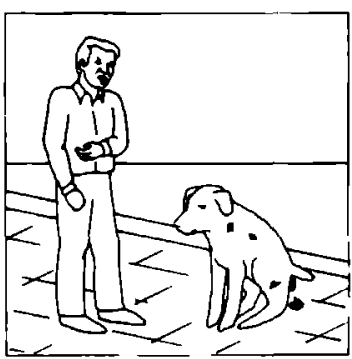

Daddy thinks it verty dirty that the filthy dog is shitting again Look at daddy's face Yich, such a dirty dog
(Interest)

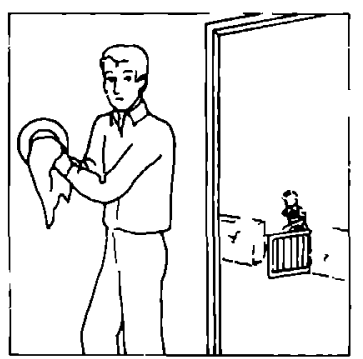

Daddy hears the garden gate and wonders if that's Johnny coming back from school Look at daddy's face ' $\mathrm{Mm}$, I wonder if that's Johnny", father thinks (joy)

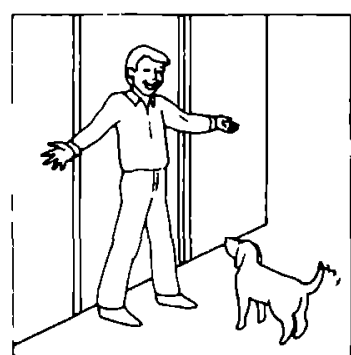

Daddy is really glad that the dog has come back home Look at daddy's face, how happy he feels Hıp, hip, hurrah, the dog has come back

(fear)

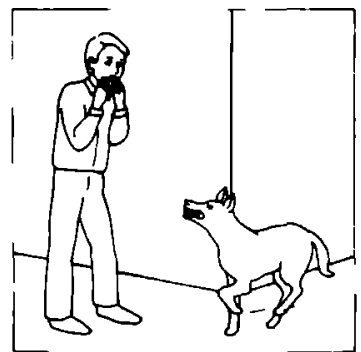

Daddy sees the big mean wolf coming around the comer again Look at daddy's face, how scared he is Oh my god, there's the wolf Look at his teeth (sadness)

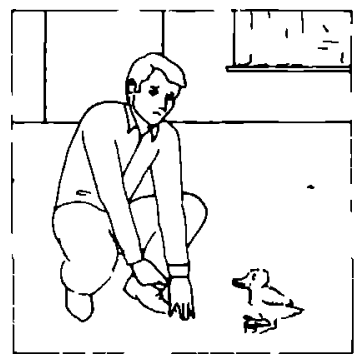

Daddy feels like crying when he sees the poor duckling again Its foot is badly hurt Look at daddy's face, how sad he feels Oh, what a poor ducklıng (anger)

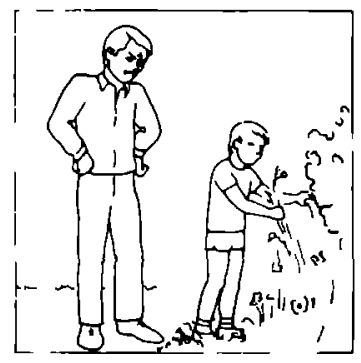

Daddy finds it very naughty that Steve is trampling on his plants He does it all the tume Look at daddy's face, how angry he is Humph, such a naughty boy

Figure 8 I Drawings with explanations representıng six emotions 
the experiment proper but not comprised in the test material. The drawings were also shown. Thus, the whole group had some idea of what was going to happen to the children which, on the basis of their date of birth, had been selected as subjects.

The ten subjects were tested individually. The stimuli had been put in random order, separately for the male and female speakers. For half of the subjects the recordings of the female voices were played first and those of the male voices second; for the other half of the subjects the order was reversed. Since too much theoretical information on the task beforehand would have been confusing, the preliminary instructions, which were given by one of the experimenters while being seated next to the subject, were kept very short. They were as follows, "Look at these drawings. In each of them you see a mommy and a daddy. They are the same mommy and daddy you just heard on the tape recorder. Now they are going to say some more things. Listen very carefully and try to find out how they feel, whether they are happy, or sad, or perhaps scared ..."

The task was practiced by means of three test stimuli. It soon appeared that some subjects experienced problems if the experimenter tried to force a choice among the drawings by asking, for example, "Is it this mommy, or is it this mommy, or is it this mommy?" (pointing at the different drawings). In other words, the subjects seemed to be confused by or-or questions. The strategy which worked best, and which was consistently applied during the scoring of the twelve experimental stimuli, was the following: First, one experimenter explained the three drawings. After the stimulus had been played three times she then asked, "Guess which mommy/daddy it is" or "Point at the mommy/daddy on the tape" or "Which drawing is it?" Finally, the stimulus was played a fourth time, after which the child was prompted to make a choice.

When it was clear that the task was understood - much to our surprise all subjects that had been selected for participation in the experiment seemed to grasp what was expected of them - the twelve experimental stimuli were played. One experimenter kept repeating the explanations with each drawing. However, after the first six stimuli had been presented a reduced form was used in order to avoid boredom and fatigue (by then the subjects knew the explanations almost by heart). The other experimenter operated the tape recorder and noted down which drawing the subjects pointed at. The test took 20 minutes.

\subsection{RESULTS}

In Table 8.1 the total number of correct identifications is given for each of the six emotions studied, for the girls and the boys separately, and for the girls and the boys together. A binomial estimate of probability was used to determine whether the various emotions were identified beyond chance 
expectancy. It may be seen from Table 8.1 that for the total group each of the six emotional categories was correctly identified at a level of significance beyond $5 \%$. Thus, the first question formulated in the introduction, as to whether $3 \frac{1}{2}$-year-old children are able to identify vocal expressions of emotion beyond chance, has to be answered positively.

The data in Table 8.1 further reveal that on the whole the difference in recognition rate between girls and boys is negligible, except, perhaps, for the emotion of anger, which was recognized by the boys twice as often as by the girls. The outcome of a $t$-test on the mean number of correct scores per emotion separated for the two sexes was not significant. Thus, the second question, as to whether there is a difference between $31 / 2$-year-old girls and $31 / 2$-year-old boys in the accuracy with which they identify vocal expressions of emotion, has to be answered negatively.

In order to answer the third question, as to whether the confusions made by the toddlers were similar to the ones made by adults, use was made of a chi square test to which Yates' correction for continuity was applied (Ferguson, 1976, p. 201-202). It appeared that when making a mistake the children chose significantly more often $\left(\chi^{2}(2)=6.88, p<.01\right)$ the distractor related to the key than the distractor not related to the key (thirty and twelve times, respective1y). Since the relationships among the emotions had been based on the classificatory behaviour of adults, it may therefore be concluded that the third question has to be answered positively: $3 \frac{1}{2}$-year-olds tend to make confusions within the same clusters as adults.

Inspection of the confusions further revealed that out of the twelve times that an emotion was mistaken for one not belonging to the same cluster, eight related to confusions among positive and active negative emotions, three to confusions among positive and passive negative emotions, and one to a

Table 8. I Number of correct identıfications of six emotions by $3 \frac{1}{2}$-year-old children

\begin{tabular}{lccl}
\hline & Males & Females & Males+Females \\
\hline Disgust & 4 & $6^{*}$ & $10^{*}$ \\
Interest & $6^{*}$ & 5 & $11^{* *}$ \\
Joy & $7^{* *}$ & $6^{*}$ & $13^{* *}$ \\
Fear & $6^{*}$ & $6^{*}$ & $12^{* *}$ \\
Sadness & $10^{* * *}$ & $10^{* * *}$ & $20^{* * *}$ \\
Anger & $8^{* *}$ & 4 & $12^{* *}$ \\
Mean & $6.8^{*}$ & $6.2^{*}$ & $13^{* * *}$ \\
\hline
\end{tabular}

Note. The maxımum score for the left and middle columns $=10$, the maximum for the right column $=20$

- $p<05$

* $p<.01$

$* * * p<.001$ 
confusion among a passive negative and an active negative emotion $\left(\chi^{2}=(2)\right.$ : $4.68, .05<p<.10$ ). Since the positive emotions included in the present study may be considered to be active rather than passive, this means that if confusions between clusters are made there is a tendency for them to be characterized by similarity in level of activity rather than by similarity in evaluative meaning.

\subsection{DISCUSSION}

The present experiment revealed that $3 \frac{1}{2}$-year-olds are able to correctly identify the vocal expressions of disgust, interest, joy, fear, sadness, and anger beyond chance expectancy. The expressions of sadness were even identified correctly $100 \%$ of the time. This means that Dimitrovsky's (1964) assumption that children have already undergone a fair amount of development in the recognition of vocally expressed emotions before age 5 is confirmed. It may even be stated that the ability to distinguish vocal expressions of at least the six emotions studied in the present experiment must be acquired before age $3 \frac{1}{2}$. With respect to vocal expressions of emotion this is the earliest age reported to date.

With respect to facial expressions of emotion, Fechner (1978) found that happy, sad, mad, and scared expressions were fully recognized at age 3. There are even indications that four-month-old infants are able to distinguish happy from angry and neutral expressions (LaBarbera, Izard, Vietze, \& Parisi, 1976), and that three-month-old infants are able to distinguish surprise from happy and, sometimes, from sad expressions (Young-Browne, Rosenfeld, \& Horowitz, 1977). In the last two experiments discrimination was assessed by using some kind of visual fixation measure. Of course, in research into vocally expressed emotions this method is ineffective. This might be one of the reasons why experimental studies in this area have been limited to older children.

No significant overall difference was found between the performance of the $31 / 2$-year-old girls and the $31 / 2$-year-old boys. This finding does not agree with the finding reported by Dimitrovsky (1964) that in her experiment the five-year-old boys did better than the girls, nor with the finding reported by Fechner (1978) that between age $2 \frac{1}{2}$ and 7 the girls did significantly better than the boys. The inconsistency of the results and the fact that in each study only a few subjects were included make it hazardous to draw any conclusions. The data being so scanty, we also think it premature to evaluate the theories which have been put forward in the past either to account for the absence of a female advantage (Dimitrovsky, 1964) or the presence of a female advantage (Blanck et al., 1981) among preschool children. (For more details with respect to this controversy, see Section 7.4.2.) 
It finally appeared that $3 \frac{1}{2}$-year-olds tend to make the same confusions as adults, i.e., tend to confuse interest and joy, disgust and anger, and sadness and fear with one another. It was shown, in other words, that confusions tended to occur within the clusters of positive, active negative, and passive negative emotions, respectively. It thus looks as if the categorization of the stimuli can be accounted for by the functioning of at least two distinctions, namely passive vs. active and negative vs. positive. The same findings were obtained in our developmental study (Chapter 7 ) with children between age 5 and 11.

The fact that in the case of confusions between clusters (active) positive and active negative emotions were confused most often suggests that in the categorization of emotions similarity in level of activity is assigned a somewhat heavier weight than similarity in evaluative meaning. This finding is again consistent with the results of our developmental study and the results of Dimitrovsky's (1964) study. In the latter, however, the tendency was far more pronounced. 


\section{Recognition by Dutch, Taiwanese, and Japanese subjects}

\subsection{INTRODUCTION}

In Chapter 6 the recognition of Dutch vocal expressions of emotion by Dutch adults was examined. In the present chapter their performance is compared with the performance of two foreign groups of subjects from Taiwan and Japan.

In contrast to vocal expressions of emotions, quite a few studies have been conducted which aimed at determining to what extent facial expressions of emotion are culture specific and to what extent they are characterized by features which are cross-culturally or even universally recognizable. Experiments in which carefully selected photographs were shown to literate as well as visually isolated, preliterate people of widely varying cultural backgrounds suggest that although there are differences in the kind of events that will elicit particular emotions and culturally determined display rules, i.e., cultural differences in the way in which emotional expressions are managed and controlled in given social settings, the facial expressions of at least some emotions seem to be universally similar and universally recognizable (Boucher \& Carlson, 1980; Ekman, 1973; Izard, 1971). Among these emotions are happiness, sadness, anger, and probably also disgust, fear, surprise, interest, shame, and contempt. Ekman explains these findings by postulating a "facial affect program", located within the nervous system of all human beings, linking particular facial muscular movements with particular emotional experiences. Izard's ideas are along the same lines. However, while Izard appears to equate "cross-cultural" with "innate" ("the data [with respect to cross-cultural recognition] ... provide a sound basis for inferring that the fundamental emotions are subserved by innate neural programs" (1977, p. 6)), Ekman is more careful. He states that "universality requires only the postulate that whatever source is responsible for the origin of facial expressions, it must be constant, not variable, for mankind. Inheritance is one such source, and a likely one; but other constants that all human beings experience in their interactions with their environment may possibly be the source of universal facial expressions" (1973, p. 171). Ekman thinks that both the innate and the environmental explanations are valid, depending on the particular expression at hand. However, he claims that more data are needed, derived, for example, from the facial expressions found among the - 
congenitally - blind, before the question as to the origin of facial expressions of emotion can be answered more definitely and in greater detail.

The extremely limited data available on the cross-cultural recognizability of vocal expressions of emotion are less conclusive. Albas, McCluskey, and Albas (1976) had forty Anglo-Canadians and forty Cree-Indians identify emotional expressions of happiness, sadness, love, and anger, produced by six Anglo-Canadian and six Cree-Indian adult males. The speakers were allowed to speak any two sentences that came to mind (in their native language) in an attempt to express each emotion. Afterwards, the utterances were rendered unintelligible by means of a lowpass filter (for an explanation and the effect of this content masking technique, see Section 2.1). The Anglo-Canadian subjects identified the Anglo-Canadian expressions more accurately than did the Indian subjects ( $80 \%$ vs. $58 \%$ ), whereas the Indian subjects identified the Indian expressions more accurately than did the Anglo-Canadian subjects ( $79 \%$ vs. $58 \%$ ). This finding corresponds to the general trend which emerged from the cross-cultural research into facial expressions of emotion, namely that cross-cultural recognition is possible, but that the recognition rate will drop. (Unfortunately, the analysis of variance carried out by Albas et al. did not contain the factor emotion, so that, the raw scores per emotion not being reported either, it is not possible to know whether there were any significant emotion $\times$ culture of speaker or emotion $X$ culture of listener interactions.)

The outcomes of other studies on the recognition of vocally expressed emotions (Beier \& Zautra, 1972; McCluskey, Albas, Niemi, Cuevas, \& Ferrer, 1975), however, show that cross-cultural distance between encoder and decoder does not necessarily lead to a loss of emotional information. Beier and Zautra (1972) compared the ability of American English, Polish, and Japanese students to correctly identify American English expressions of six emotions and attitudes (happiness, fear, sadness, anger, indifference, and firt) produced with standard speech samples of four different lengths ("hello", "good morning", "how are you", and a sentence). It was found that the Polish and Japanese subjects increased their accuracy as the length of the expressions increased until their scores were comparable to the American English ones. Thus, in this study "foreigners" performed just as well as "compatriots" provided the emotional expressions were long enough.

The results obtained by McCluskey et al. (1975) go even further than this. They compared the performance of Canadian and Mexican children (ranging from 6 to 11 years) when asked to identify vocal expressions of happiness, sadness, love, and anger produced by Canadian and Mexican actresses. It was found that the Mexican children were significantly more accurate than their Canadian counterparts, not only in the identification of the Mexican expressions but in the recognition of the Canadian expressions as well. This shows that "foreigners" may even exceed the performance of "compatriots" in the identification of vocal expressions of emotion. 
Although it thus appears that the amount of emotional information preserved in a cross-cultural setting varies from one experiment to another, in general it has been found that the vocal expressions of most emotions investigated so far are recognizable beyond chance expectancy. This outcome suggests that there may be universal vocal patterns of emotion in addition to a number of universal facial patterns of emotion.

Moreover, there is some evidence that there may be common patterns of errors in the identification of vocal expressions of emotion across cultures, a finding that is of interest in connection with the possible existence of universal emotional dimensions. Thus, Albas et al. (1976) found that both AngloCanadian and Cree-Indian subjects often confused the active emotions of happiness and anger on the one hand, and the passive emotions of sadness and love on the other, regardless of the culture of the speakers. This suggests, as has been reported before for American English (Dimitrovsky, 1964; Davitz, 1964c), that in general errors in the identification of vocal expressions of emotion seem to be a function of similarity in level of activity of the emotions concerned rather than, for example, similarity in evaluative meaning (see also Chapters 7 and 8). Another aspect of confusions which has been stated for different cultures is the fact that confusions sometimes tend to be asymmetrical. Kramer (1964), for example, reports that American English as well as Japanese expressions of anger were mistaken for contempt much more often that the other way round. A similar finding was reported in connection with love and indifference.

Unfortunately, the above findings with regard to the cross-cultural recognition of vocal expressions of emotion are based on quite limited data. Thus, it seems evident that much more research should be conducted in many more cultures with respect to a greater variety of emotions.

In the present chapter the performance of groups of Dutch, Taiwanese, and Japanese subjects in decoding Dutch vocal expressions of emotion is compared. The data pertaining to the Dutch subjects have been derived from the experiment reported on in Chapter 6. The two groups of foreign subjects were chosen on the one hand on theoretical grounds, because the Japanese and Taiwanese languages are unrelated to one another as well as to Dutch, and on the other on pragmatic grounds, because the present author at the time happened to have connections in Taiwan and Japan who were interested in and able to participate in the research.

The aim of the study was to answer the following questions:

(1) Do Dutch, Taiwanese, and Japanese adults differ in their ability to identify Dutch vocal expressions of emotion?

(2) Are there similar patterns in the confusions made by Dutch, Taiwanese and Japanese subjects?

Doing so, we hoped to gain insight into the question of whether or not vocal expressions of discrete emotions are cross-culturally recognizable, and to collect evidence on the universality of emotional dimensions. Data in this 
area are essential in determining whether the vocal characteristics of emotions, just as has been claimed for their facial characteristics, could be physiologically determined and, perhaps, be innate.

\subsection{METHOD}

Recognition was assessed by means of a forced judgment task in which the subjects responded to vocal expressions of emotion by choosing among emotion labels.

\subsubsection{Subjects}

Forty-eight language students (twenty-four males and twenty-four females) attending a Teachers' Training College in Nijmegen took part in the Dutch recognition experiment. The mean age was 20 years and 6 months varying from 18 to 28 years. The subjects were paid for their participation.

Forty students from National Cheng Kung University of Tainan took part in the Taiwanese recognition experiment: ten males studying English, ten females studying English, ten males studying Chinese and ten females studying Chinese. The mean age of the students was 22 years and 6 months, ranging from 21 years to 28 years. The subjects were not paid for their participation. In general the inhabitants of Taiwan may be said to have little exposure to spoken Western languages produced by native speakers. The teaching of English in middle and high schools is mainly restricted to translation into Chinese, and there are few opportunities, especially in the South of Taiwan, where Tainan is located, to meet foreigners.

Forty-one male high school students from Tokyo took part in the Japanese recognition experiment. The mean age was 16 years and 9 months, ranging from 16 to 17 years. The subjects were not paid for their participation. They had had little exposure to spoken English and none to other western languages.

\subsubsection{Speech material}

The speech material consisted of eighty emotional expressions, namely eight speakers (four males and four females) $X$ one phrase ( $t$ wee maanden zwanger) $X$ ten emotions (neutral [NE], disgust [DI], surprise [SU], shame [SH], interest [IN], joy [JO], fear [FE], contempt [CO], sadness [SA], and anger [AN]). (For a detailed description of the speech material, see Chapter 2.)

\subsubsection{Procedure}

The procedure followed in the Dutch, Taiwanese, and Japanese experiments was similar: In all three cases the subjects were seated in a language laborato- 
ry, where they listened to the recordings via headphones, and in all three cases a forced judgment task was used with ten alternatives. The experiments differed in that the Taiwanese and Japanese subjects were offered 80 emotional realizations of one standard phrase (twee maanden zwanger), whereas the Dutch subjects responded to 320 utterances of which the 80 twee maanden zwanger were a subset (we thought we could not possibly ask our friends in Taiwan and Japan to run a two-hour-experiment; judgment of the 80 stimuli took 25 minutes).

Before listening to the experimental stimuli the subjects received a booklet containing rating sheets and instructions. The Dutch, Taiwanese, and Japanese instructions were the same except, of course, for the language in which they were written and the fact that the differences in setup mentioned above were taken into account. For the preparation of the Taiwanese and Japanese translations care was taken to involve several native speakers and linguists in the selection of the best possible equivalents of the Dutch emotion labels. The translation did not prove to be a problem. For the English translation of the Dutch version, see Appendix B. The English translation of the Taiwanese and Japanese version was as follows:

If you want to know how someone feels you can derive this not only from what someone says but also from how he or she says it. Thus, the way in which an utterance is spoken contains information about the emotion one is experiencing. By means of this experiment we want to find out to what extent people, solely on the basis of intonation, are able to determine the emotion with which an utterance has been emitted. By the term "intonation" we do not only refer to pitch or changes in pitch, but also to such speech characteristics as tempo or voice quality. In a moment you are going to hear a number of recordings of women and men producing the same phrase with ten different emotions, namely disgust, surprise, shame, interest, joy, neutral, fear, contempt, sadness, and anger. We ask you to listen very carefully to the recordings - you will not be able to understand what is being said, so only pay attention to how the phrases are being said - and determine which emotion the speaker has tried to express. You indicate your choice by encircling one and only one emotion for each utterance on your score sheet (the numbers on the sheet correspond with the numbers preceding the utterances on the tape). You will first hear six utterances to practice. After you have rated these practice utterances on your form there will be time for you to ask questions in case something is not clear. After that you will hear the proper set of eighty utterances.

\subsection{RESULTS}

First, the percentages of correct responses of the three subject groups were compared. Subsequently, an analysis of the incorrect responses was undertaken by examining symmetrical and asymmetrical confusions among emo- 
tions, and by carrying out a hierarchical cluster analysis and multidimensional scaling. Hierarchical cluster analysis was used to extract information about groupings of emotions and their internal structure. Multidimensional scaling was used to represent confusions among emotions in terms of distances in a Euclidean space.

Before the results are presented, it should be noted that analyses of variance (repeated measurements design) were carried out on the scores of the three subject groups in order to assess whether there was an effect of the main factor of sex of speaker (for all three subject groups) and the subject variables of sex (for the Dutch and Taiwanese subjects) and study (for the Taiwanese subjects: students of English/students of Chinese). No significant effects $(p<.05)$ were found. Therefore, use was made of the summed scores in the subsequent analyses mentioned below.

\subsubsection{Rate of recognition}

The first question formulated in the introduction, as to whether Dutch, Taiwanese, and Japanese subjects differ in their ability to identify Dutch expressions of emotion, was answered positively. In Tables 9.1, 9.2, and 9.3 the mean percentages of each of the ten emotional categories are given separately for each of the three subject groups on the diagonals of each of the three matrices. It may be seen that for all emotions the Dutch subjects did best, although it must also be noted that even they scored hardly $50 \%$ correct for the categories of fear and contempt. The difference in rate of recognition between the Dutch and the Taiwanese subjects, and that between the Dutch and the Japanese subjects, both tested by means of a $t$-test, was significant ( $t$

Table 9. I Dutch recognition experiment: Confusion matrix for ten emotions (in \%)

\begin{tabular}{lcrrrrrrrrr}
\hline Stimuli & \multicolumn{1}{l}{ Responses } & & & & & & & \\
\cline { 2 - 10 } & NE & DI & SU & SH & IN & JO & FE & CO & SA & AN \\
\hline Neutral & 87 & 0 & 0 & $4^{*}$ & 1 & 0 & 0 & 2 & $4^{*}$ & 2 \\
Disgust & 1 & 55 & 1 & 3 & 1 & 3 & 1 & $24^{*}$ & 6 & 4 \\
Surprise & 0 & 3 & 68 & 0 & $8^{*}$ & $7^{*}$ & 2 & $6^{*}$ & 0 & 5 \\
Shame & $12^{*}$ & 1 & 0 & 61 & 0 & 0 & $8^{*}$ & 2 & $15^{*}$ & 0 \\
Interest & 2 & 0 & $24^{*}$ & 0 & 69 & 0 & 0 & 5 & 0 & 0 \\
Joy & 1 & 1 & $13^{*}$ & 0 & 1 & 76 & 1 & 3 & 0 & 3 \\
Fear & 0 & 2 & $14^{*}$ & 5 & 2 & 2 & 51 & 2 & $20^{*}$ & 3 \\
Contempt & 0 & $20^{*}$ & 0 & 3 & 1 & 0 & 0 & 48 & 1 & $27^{*}$ \\
Sadness & 0 & 2 & 0 & $15^{*}$ & 0 & 1 & 4 & $5 *$ & 73 & 0 \\
Anger & 1 & $8^{*}$ & 5 & 1 & 1 & 2 & 2 & $11^{*}$ & 0 & 70 \\
Total & 104 & 92 & 126 & 94 & 83 & 92 & 69 & 109 & 119 & 113 \\
\hline
\end{tabular}

$p<.05$ (see text) 
Table 9.2 Taiwanese recognition experıment: Confusion matrix for ten emotions (in \%)

\begin{tabular}{lcccccccccc}
\hline Stımult & \multicolumn{1}{l}{ Responses } & & & & & & & & \\
\cline { 2 - 10 } & NE & DI & SU & SH & IN & JO & FE & CO & SA & AN \\
\hline Neutral & 53 & $9^{*}$ & 2 & 6 & 4 & 2 & 1 & $11^{*}$ & $8^{*}$ & 3 \\
Disgust & 10 & 26 & 2 & 5 & 2 & 4 & 8 & $17^{*}$ & 10 & $16^{*}$ \\
Surprise & 2 & 4 & 53 & 1 & $17^{*}$ & $12^{*}$ & 1 & 6 & 0 & 4 \\
Shame & $23^{*}$ & 8 & 2 & 30 & 2 & 0 & 8 & 6 & $21^{*}$ & 0 \\
Interest & $13^{*}$ & 3 & $29^{*}$ & 2 & 31 & 4 & 2 & $12^{*}$ & 1 & 2 \\
Joy & 1 & $12^{*}$ & $13^{*}$ & 2 & 10 & 24 & $13^{*}$ & 6 & 3 & $14^{*}$ \\
Fear & 4 & 6 & $20^{*}$ & 7 & 5 & 5 & 36 & 4 & 6 & 7 \\
Contempt & 10 & $24^{*}$ & 3 & 10 & 4 & 8 & 2 & 19 & 6 & $13^{*}$ \\
Sadness & 7 & $8^{*}$ & 0 & $12^{*}$ & 1 & 1 & $10^{*}$ & 4 & 53 & 3 \\
Anger & 2 & $11^{*}$ & $10^{*}$ & 1 & 4 & 7 & 6 & $11^{*}$ & 3 & 47 \\
Total & 125 & 112 & 134 & 76 & 80 & 68 & 88 & 96 & 112 & 110 \\
\hline
\end{tabular}

$* p<05$ (see text)

Table 9.3 Japanese recognitıon experıment: Confusion matrix for ten emotions (in \%)

\begin{tabular}{|c|c|c|c|c|c|c|c|c|c|c|}
\hline \multirow{2}{*}{ Stımulı } & \multicolumn{10}{|c|}{ Responses } \\
\hline & NE & $\mathrm{DI}$ & SU & SH & IN & Jo & FE & $\mathrm{CO}$ & SA & AN \\
\hline Neutral & 56 & $15^{*}$ & 1 & 4 & 8* & 1 & 1 & $10^{4}$ & 3 & 1 \\
\hline Disgust & 10 & 26 & 4 & 8 & 4 & 3 & 6 & $18 *$ & 13* & 9 \\
\hline Surprise & 6 & 3 & 29 & 2 & $17^{*}$ & $22^{*}$ & 3 & $14^{\circ}$ & 2 & 2 \\
\hline Shame & $23^{*}$ & $20^{*}$ & 2 & 14 & 3 & 3 & 5 & 7 & $24 *$ & 0 \\
\hline Interest & $24^{*}$ & 4 & 7 & 3 & 38 & 8 & 1 & $15^{*}$ & 1 & o \\
\hline Joy & 3 & 5 & $20^{*}$ & 3 & 7 & 20 & $18^{*}$ & 6 & 8 & 11 \\
\hline Fear & 4 & 6 & $19^{*}$ & 4 & 5 & 5 & 19 & 4 & $28^{*}$ & 7 \\
\hline Contempt & $21^{*}$ & $16^{*}$ & 3 & 6 & 6 & 4 & 3 & 22 & 9 & 11 \\
\hline Sadness & $6 *$ & $6^{*}$ & 3 & 4 & 2 & 0 & 3 & 2 & 70 & 3 \\
\hline Anger & 2 & 7 & $12^{*}$ & 2 & 6 & 3 & $10^{*}$ & $13^{*}$ & 4 & 40 \\
\hline Total & 155 & 108 & 99 & 50 & 96 & 70 & 67 & 110 & 162 & 83 \\
\hline
\end{tabular}

$-p<05$ (see text)

(9) $=8.01, p<.001$ and $t(9)=7.34, p<.001$, respectively). The difference between the Taiwanese and Japanese subjects was not significant $(t(9)=0.93$ $p>$.10). Although it thus appears that in the cross-cultural setting a fair amount of emotional information got lost, a binomial estimate of probability revealed that the Taiwanese were still able to identify all, and the Japanese all but one of the emotions beyond chance at a $5 \%$ level of significance. This one exception pertains to the recognition of shame.

It may further be seen that there are substantial differences in the percentages 
of correct identification for the different emotions. Some emotions occupy roughly the same rank order position in all three experiments. Thus, it appears that neutral, sadness, and anger were generally fairly easy to recognize whereas disgust, shame, and contempt were generally rather difficult. Interest occupies a mid position for all groups. The relative recognizability of the other emotions, however, appears to be less consistent. Joy, for example, which came out as the second best recognized emotion in the Dutch experiment, came out as the second worst recognized emotion in the Taiwanese experiment and the third worst recognized emotion in the Japanese experiment.

On the other hand, a look at the column totals in Tables 9.1, 9.2, and 9.3 reveals that not all response categories have been chosen equally often. It may well be that the low score for joy by the Taiwanese and Japanese subjects can, at least partly, be accounted for by the fact that for them joy was one of the least favoured response categories, thus diminishing the chance of a correct response. Similarly, the high recognition scores for neutral and sadness by the Japanese may be partly due to the fact that these response categories have been chosen far more often than chance expectancy, thus increasing the probability for a response to be correct. In order to gain insight into the influence of the response biases on the number of correct responses, a formula was applied to the raw percentages which takes into consideration the number of times a particular emotional category has been chosen as a response (for the exact computation, see footnote b to Table 6.3). The resulting percentages are given in Table 9.4, in addition to the uncorrec-

Table 9.4 Mean percentages of correct identıfications of ten emotions by three subject groups, uncorrected (Unc), corrected for chance (CfC), and corrected for response bias (CFB)

\begin{tabular}{|c|c|c|c|c|c|c|c|c|c|}
\hline & \multicolumn{3}{|c|}{ Holland } & \multicolumn{3}{|l|}{ Taiwan } & \multicolumn{3}{|l|}{ Japan } \\
\hline & Unc & $\mathrm{CrC}$ & CIB & Unc & $\mathrm{CfC}$ & $\mathrm{CtB}$ & Unc & $\mathrm{CfC}$ & $\mathrm{CfB}$ \\
\hline Neutral & $87(1)$ & 86 & $85(1)$ & $53(2)$ & 48 & $48(2)$ & $56(2)$ & 51 & $47(2)$ \\
\hline Disgust & $55(8)$ & 50 & $57(9)$ & $26(8)$ & 18 & $25(9)$ & $26(6)$ & 18 & $25(6)$ \\
\hline Surprise & $68(6)$ & 64 & $61(8)$ & $53(2)$ & 48 & $46(3)$ & $29(5)$ & 21 & $29(5)$ \\
\hline Shame & $61(7)$ & 57 & $63(6)$ & $30(7)$ & 22 & $35(6.5)$ & $14(10)$ & 4 & $21(95)$ \\
\hline Interest & $69(5)$ & 66 & $76(3)$ & $31(6)$ & 23 & $35(6.5)$ & $38(4)$ & 31 & $39(4)$ \\
\hline Joy & $76(2)$ & 73 & $79(2)$ & $24(9)$ & 16 & $30(8)$ & $20(8)$ & 11 & $24(7.5)$ \\
\hline Fear & $51(9)$ & 46 & $62(7)$ & $36(5)$ & 29 & $38(5)$ & $19(9)$ & 10 & $24(7.5)$ \\
\hline Contempt & $48(10)$ & 42 & $46(10)$ & $19(10)$ & 10 & $19(10)$ & $22(7)$ & 13 & $21(9.5)$ \\
\hline Sadness & $73(3)$ & 70 & $67(4)$ & $53(2)$ & 48 & $50(1)$ & $70(1)$ & 67 & $57(1)$ \\
\hline Anger & $70(4)$ & 67 & $66(5)$ & $47(4)$ & 41 & $45(4)$ & $40(3)$ & 33 & $44(3)$ \\
\hline Grand mean & 66 & 62 & 66 & 37 & 30 & 37 & 33 & 26 & 33 \\
\hline
\end{tabular}

Note. The numbers in parentheses indicate the rank order positions from $1=$ best to $10=$ worst recognized emotion

For the formulas used to calculate the percentages corrected for chance and the percentages corrected for response bias, see notes a and b to Table 6.3 
ted percentages and the percentages corrected for chance (for the rationale behind the latter correction, see Section 6.3.1; for its computation, see footnote a to Table 6.3).

In Table 9.4 the rank orders of the recognition of the different emotions, both on the basis of the uncorrected scores and on the basis of the scores corrected for response bias, are also given (the rank order of the scores corrected for chance is not indicated, since it is - by nature - identical with the rank order of the uncorrected scores). It may be observed that correction for response bias does not result in drastic changes in the rank order positions of the various emotions: In only four cases was there a difference of two or more than two positions, namely with respect to surprise, interest, and fear in the Dutch experiment (the first one going down and the latter two going up), and with respect to contempt in the Japanese experiment. It may be observed that the rank order position of joy in Taiwan, and those of neutral, joy, and sadness in Japan - positions which we thought might have been confounded rather strongly by response bias - hardly changed as a result of the correction.

\subsubsection{Confusions}

The second question, as to whether the confusions made by the Dutch, Taiwanese, and Japanese subjects are similar, cannot be answered with a simple yes or no. Inspection of the confusion data given in the off-diagonal cells of Tables 9.1, 9.2, and 9.3 shows that within each of the three subject groups there seem to be fairly systematic tendencies for some emotions to be mistaken for one or two particular other emotions. It is also apparent that these confusions are often not symmetrical. A very clear example of such an asymmetrical confusion - and one which seems to be stable across cultures - is that between surprise and fear, the percentages of scores with surprise taken for fear being $2 \%, 1 \%$, and $3 \%$ for Holland, Taiwan, and Japan respectively, and the percentages of scores with fear taken for surprise being $14 \%, 20 \%$, and $19 \%$. A similar trend may be observed for shame and neutral.

In Figures 9.1, 9.2, and 9.3 we have tried to capture the confusions among emotions as clearly as possible, separately for the three subject groups. In these diagrams a single arrow (for example anger - surprise), means that anger was mistaken for surprise more often than surprise was mistaken for anger; it indicates, in other words, an asymmetrical relationship. Symmetrical confusions are indicated by a double arrow $(-)$. The decision to call a confusion symmetrical or asymmetrical was based on the following criterion: For each emotion in each of the three data sets the number of incorrect scores was assessed by subtracting the number of correct scores from the total number of scores for that emotion. The null-hypothesis was that the subjects, when making a mistake, did not have a preference for particular alternatives, i.e., that the incorrect scores were randomly spread over the nine alternatives from which they had to choose. By means of a binomial estimate of probabi- 


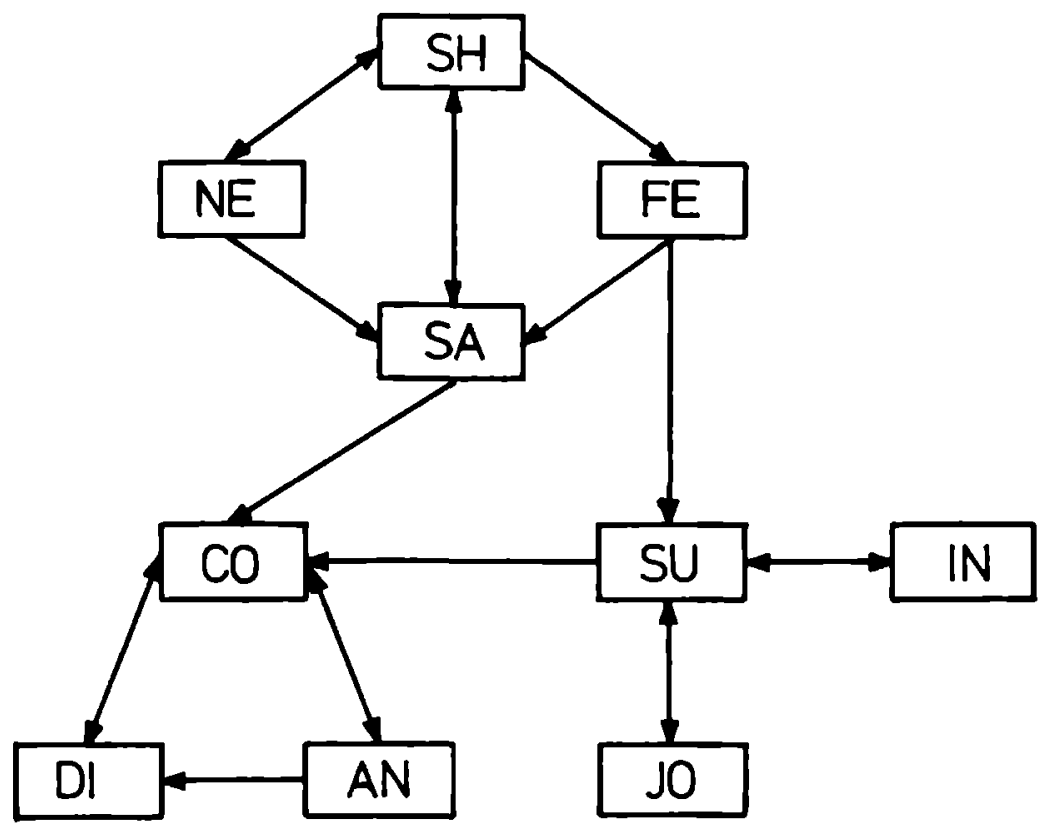

Figure 9 / Dutch recognition experiment Nature of the confusions among ten emotions (and $\longrightarrow=$ asymmetrical confusions, $\longrightarrow$ = summetrical confusion)

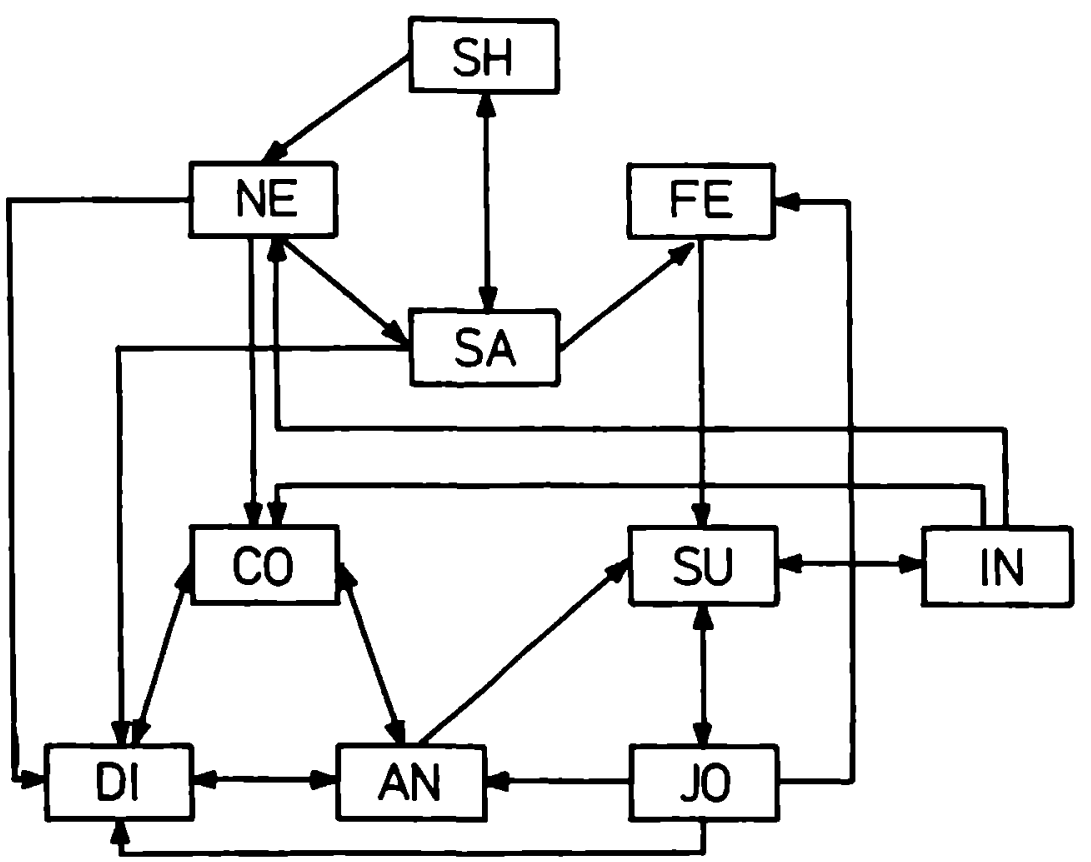

Figure 92 Taiwanese recognition experiment Nature of the confusions among ten emotions $(\longleftrightarrow$ and $\longrightarrow=$ asymmetrical confusions, $\longrightarrow \longrightarrow=$ symmetrical confusion) 


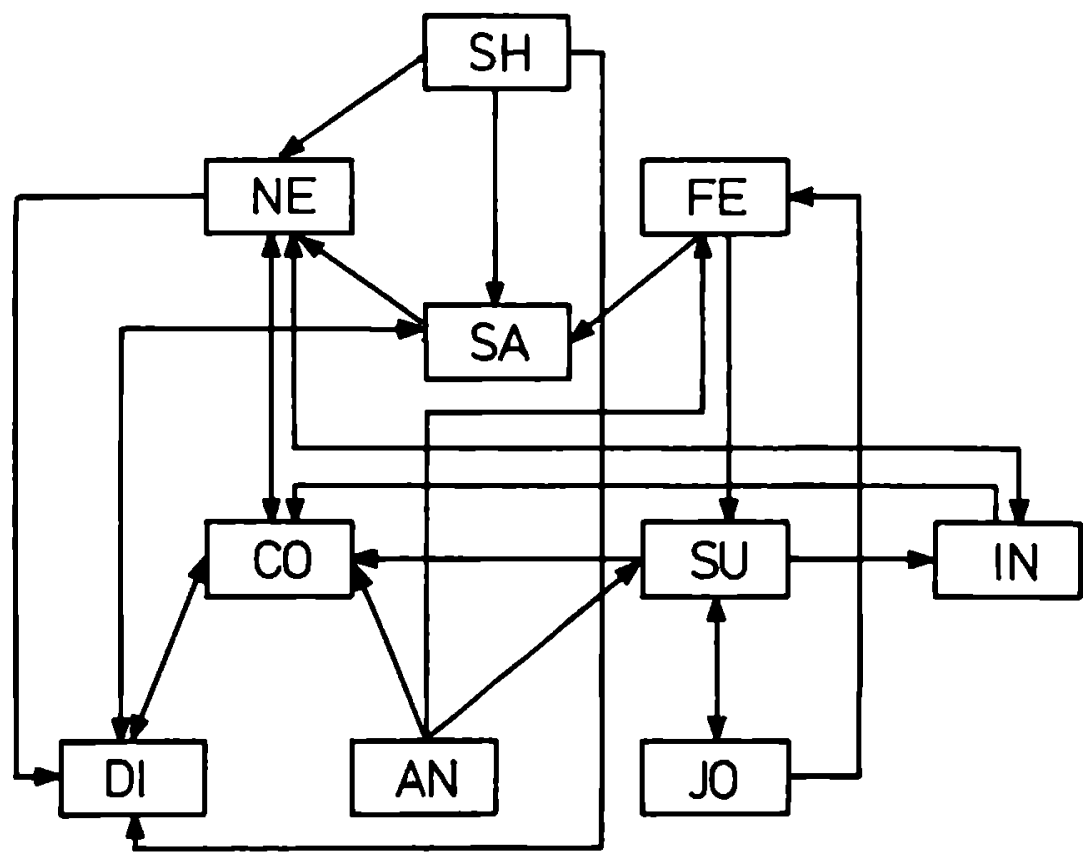

Figure 9.3 Japanese recognition experıment: Nature of the confusions among ten emotions $\longleftrightarrow$ and $\longrightarrow=$ asymmetrical confusions, $\longleftrightarrow$ symmetrical confusion)

lity it was assessed which off-diagonal cells in each row contained a number of scores exceeding chance expectancy at the 5\% level. In this way the confusion scores indicated with an asterisk in Tables 9.1, 9.2, and 9.3 were retained. A confusion was called symmetrical if both the frequency with which emotion $i$ was mistaken for emotion $j$ and the frequency with which emotion $j$ was mistaken for emotion $i$ were significant; it was called asymmetrical if only one of the two frequencies exceeded chance expectancy.

Since Dutch was the language in which the emotions were expressed, confusions were first grouped according to the Dutch subjects' responses. This layout was then preserved for the Taiwanese and Japanese responses in order to compare their confusion patterns with those of the Dutch.

It may be seen from Figures 9.1,9.2, and 9.3 that the structure of the Dutch diagram looks a lot simpler than either that of the Taiwanese or that of the Japanese diagram. This is due to the fact that the Dutch experiment yielded a smaller number of significant and a relatively greater number of symmetrical confusions than the other two experiments, thus limiting the number of connections between emotions in the diagram. In addition, the confusions yielded in the Dutch experiment look less dispersed than those yielded in the other two experiments. The Dutch subjects appear to have made most confusions within three, fairly independent clusters of emotions, the first one 
comprising neutral, shame, fear, and sadness; the second one comprising surprise, interest, and joy; and the third one comprising disgust, contempt, and anger. In fact, each of these clusters is connected to each of the others by only one asymmetrical relation. Although the structures of the Taiwanese and Japanese diagrams manifest some systematic features too - still resembling those of the Dutch diagram - the number of inter-group connections for them is larger. Still, a careful look at the diagrams reveals that most intergroup connections for the Taiwanese and Japanese groups are shared. It may further be seen that there is only one asymmetrical confusion, namely fear $\rightarrow$ surprise, and only two symmetrical confusions, namely surprise - joy and disgust - contempt, which occur in all three diagrams.

A statistically better founded technique for analyzing confusions is hierarchical cluster analysis (see Section 4.3.3). It has the drawback, however, of not being able to deal with asymmetrical relations among elements. Therefore, the confusion matrices had to be made symmetrical - this was done according to the method described by Klein, Plomp, and Pols (1970) - before the analysis could be carried out. For all three confusion matrices both the minimum method and the maximum method were applied (see Figures 9.4, 9.5, and 9.6).

The Dutch data are represented considerably better by means of the maximum method than by means of the minimum method (.77 and .63, respectively). The two Taiwanese dendrograms represent the original data equally well (.63). For the Japanese experiment, the correlation between the distances in the data matrix and the ultrametric distances is .65 for both the minimum and the maximum methods; however, the latter suffers from the so-called chaining effect.

It appears from Figures 9.4 and 9.5 that the structures of the Dutch and Taiwanese dendrograms have quite a lot in common. For both groups of
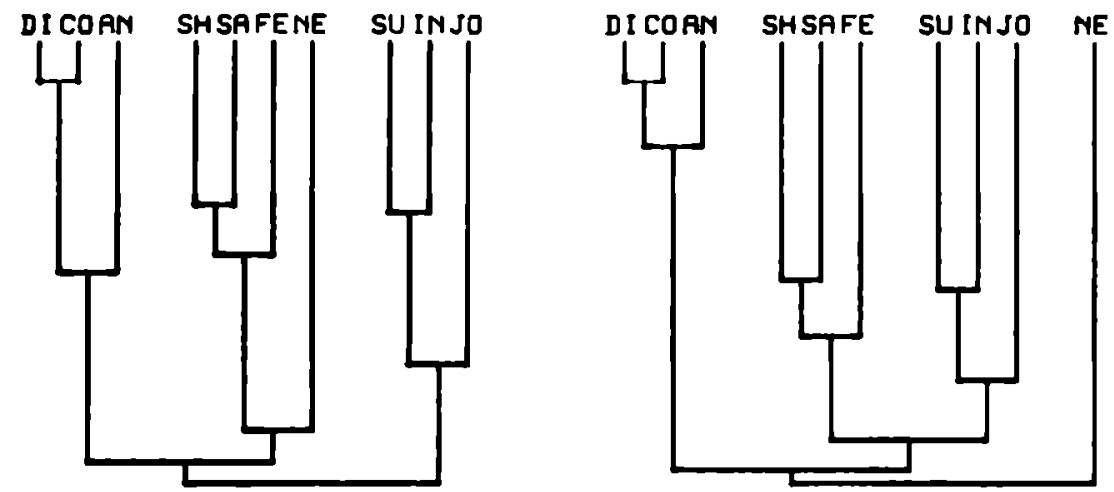

Figure 9.4 Dutch recognition experiment: Hierarchical cluster analysis for ten emotions (minimum method at the left, maximum method at the right) 

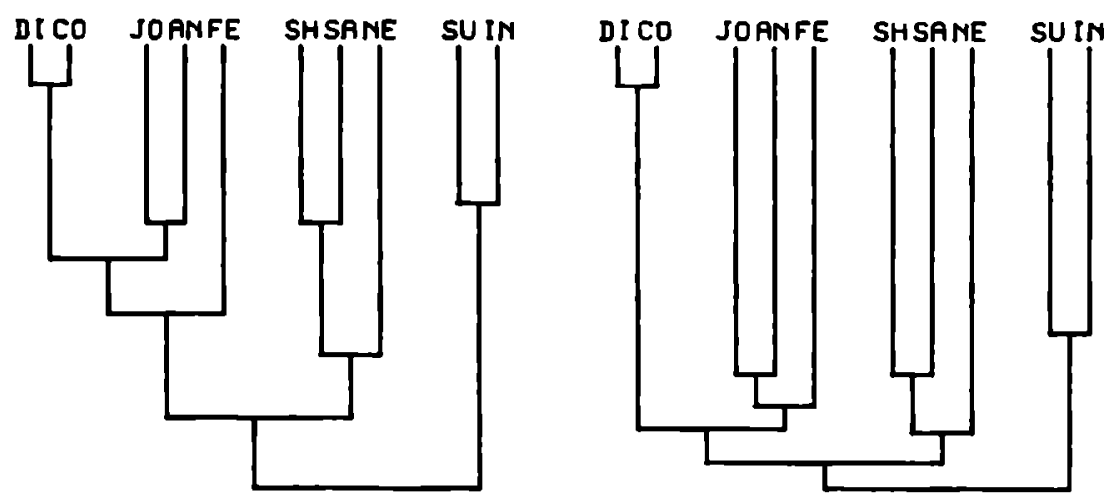

Figure 95 Taıwanese recognition experıment Hierarchıcal cluster analysıs for ten emotions (minimum method at the left, maximum method at the right)

subjects disgust and contempt are branched together, as well as shame and sadness, and surprise and interest. Also, in both pairs of trees, neutral is connected at a low level with the other emotions, which means that in neither experiment it has been confused systematically with any of the others. The only notable differences are the positions of fear and joy, which in the Dutch dendrograms are clustered together with shame and sadness, and surprise and interest respectively, whereas in the Taiwanese experiment fear and joy are clustered together with anger.

The similarity between the Dutch and the Japanese dendrograms (Figures 9.4 and 9.6) is smaller. The only clusters which they share - clusters which were also present in the Taiwanese dendrogram and are therefore common to all three experiments - are that of disgust and contempt and that of surprise and interest (the latter for Japan only in the minimum method tree). The low branching of neutral is also present in the Japanese trees. The other emotions
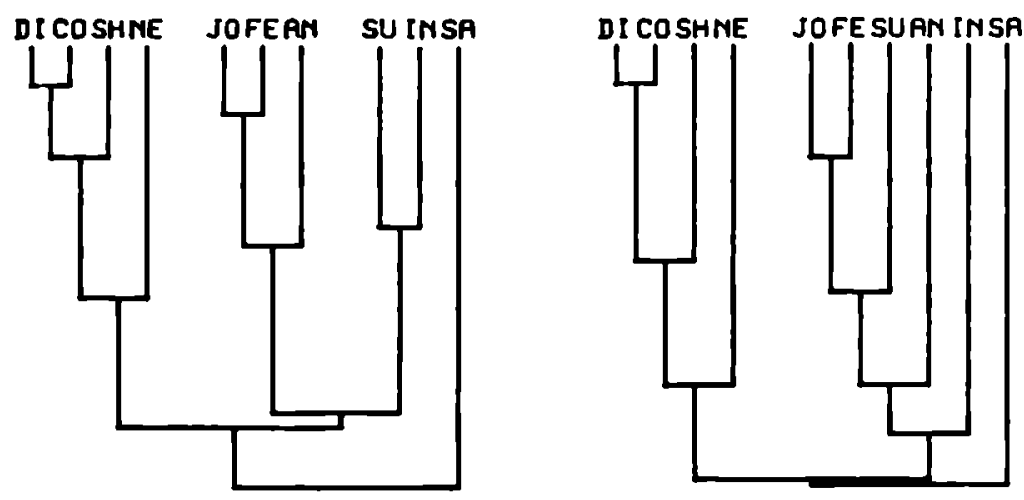

Figure 96 Japanese recognition experiment: Hierarchical cluster analysis for ten emotions (minımum method at the left, maxımum method at the right) 
are arranged differently. Note that in the Japanese tree sadness occupies an independent position.

The Taiwanese and Japanese dendrograms (Figures 9.5 and 9.6) look pretty much alike. In addition to the cluster of disgust and contempt, and that of surprise and interest, they also share the cluster of joy, fear, and anger. The Taiwanese and Japanese dendrograms differ in the position of sadness and the relation between the pair of disgust and contempt and the pair of shame and neutral.

It thus appears that there are both similarities in and differences between the confusions which the Dutch, Taiwanese, and Japanese subjects have made. Assuming that the confusions among the emotional expressions are a function of their being similar in some respect, these outcomes suggest that across the three subject groups some characteristics of the stimuli have received equal attention or have been interpreted in the same way, but others not. Or, stated in dimensional terms, it looks as if some of the dimensions along which the emotional expressions have been discriminated are common to all three subject groups, but that there are others which are specific to each of them.

A data processing technique which has been especially designed to represent the judged similarity between objects - which in our case may be derived from the number of times the emotional categories have been confused with one another (cf. Strongman, 1978, p. 215) - in terms of proximity in an $n$-dimen-

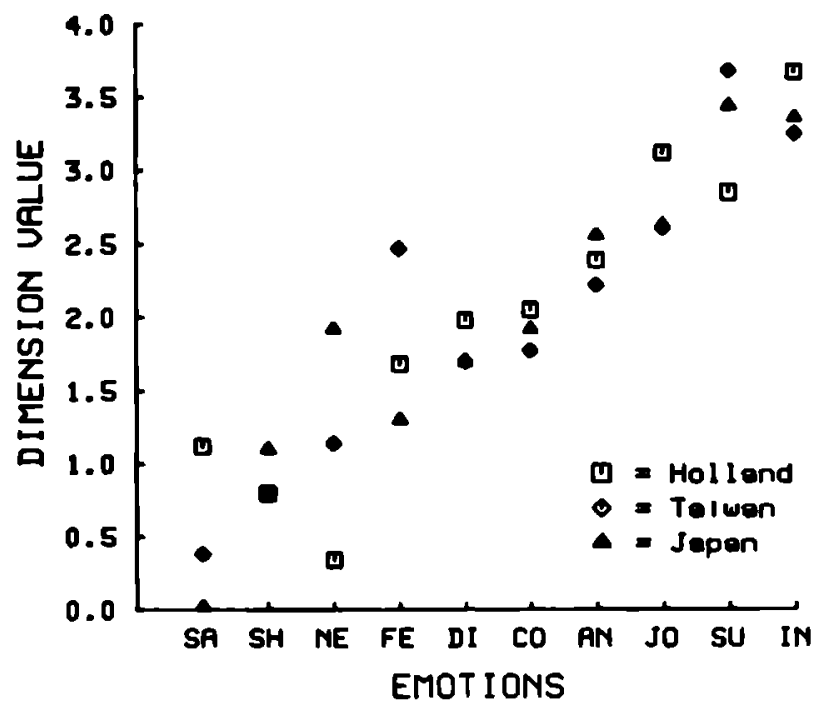

Figure 9.7 Positions of ten emotions in a one-dimensional space for three recognition experiments 
sional space, and might thereby provide insight into the nature of the dimensions or the stimulus characteristics that underlie the similarity judgments, is multidimensional scaling. Input to the program were the same symmetrized confusion data on which the hierarchical cluster analyses were carried out.

The combination of the Euclidean metric and one dimension resulted in stresses of $.21, .18$, and .32 , and the combination of the Euclidean metric and two dimensions in stresses of $.07, .00$, and .07 for Holland, Taiwan, and Japan, respectively. According to Wagenaar and Padmos (1971), this means that for all three experiments the correspondence between the one-dimensional configuration and the symmetrized confusion data as well as the correspondence between the two-dimensional configuration and the symmetrized confusion data are acceptable at a level of significance of $5 \%$. The addition of a third dimension only resulted in a slight lowering of the stress. Therefore, the three-dimensional solution will not be considered.

In Figure 9.7 the positions of the emotions in the one-dimensional solution are graphically presented. It may be seen that except for one or two emotions such as neutral and fear, all of the ten emotions occupy highly similar positions for all three experiments along the one dimension extracted. And indeed, product-moment correlations between the Dutch and Taiwanese, Dutch and Japanese, and Taiwanese and Japanese dimension values yielded coefficients of $r(8)=.84, p<.01, r(8)=.73, p<05$, and $r(8)=.87, p<.01$, respectively.

At first sight the dimension presented in Figure 9.7 may look like an evaluative dimension, with interest, surprise, and joy at the positive extreme, and shame and sadness at the negative extreme. However, the position of neutral amidst the negative emotions and the position of anger next to joy do not seem to fit in with this interpretation. More convincing is a labeling in passive/active terms, with interest, surprise, joy, and anger as the most active emotions, with contempt, disgust, and fear occupying an intermediate position, and with neutral, shame, and sadness as the most passive emotions. The positions of the emotions in the two-dimensional solution are graphically presented in Figures 9.8,9.9, and 9.10. The axes of the three configurations have been rotated orthogonally in such a way as to attain maximum similarity in the positioning of the emotions along the horizontal dimension. It may be seen that the distribution of the emotions along the dimension thus obtained has much in common with the distribution of the emotions along the dimension which emerged from the one-dimensional solution. Again, roughly three blocks of emotions may be distinguished, namely shame, sadness, and neutral on one side, disgust, contempt, and fear in the middle, and anger, interest, joy, and surprise on the other side. Thus, in the two-dimensional solution for all three subject groups an activity component appears to be present too.

The positioning of the emotions along the vertical dimension is very different for each of the three experiments, so that a common label is 


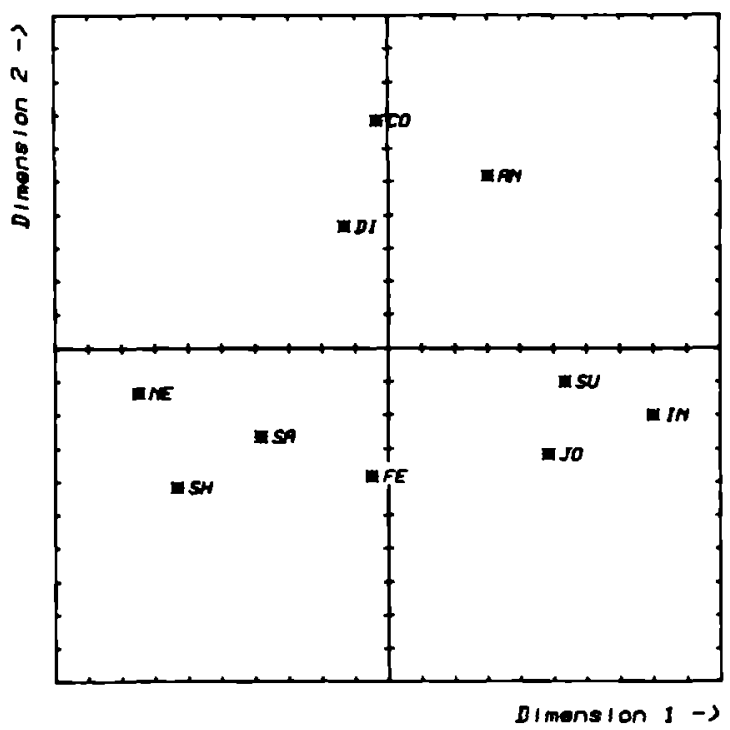

Figure 9.8 Dutch recognition experiment: Positions of ten emotions in a two-dimensional space

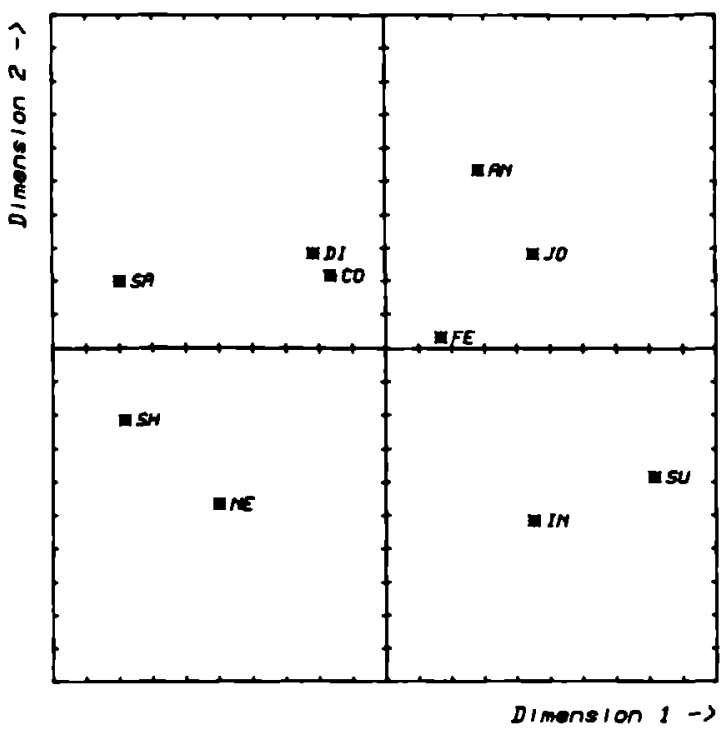

Figure 99 Taiwanese recognitıon experıment Positions of ten emotions in a two-dimensional space 


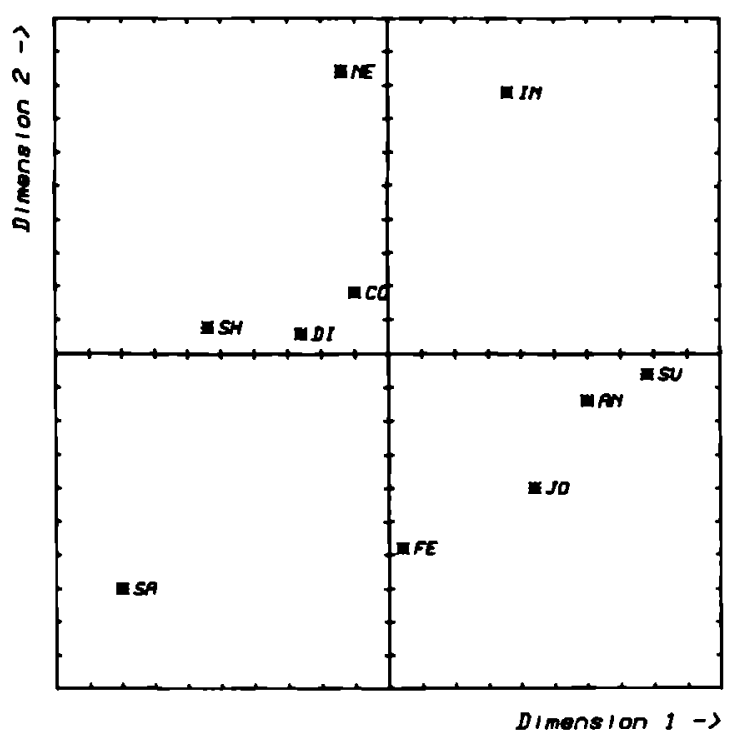

Figure 910 Japanese recognition experiment Positions of ten emotions in a two-dimensional space

impossible to give. Even when the three configurations are considered separately it is not apparent which emotional aspect this dimension represents It is, in any case, not evaluative in nature.

\subsection{DISCUSSION}

The present study revealed that although Dutch subjects on the whole did about twice as well as either Talwanese or Japanese subjects in identifying Dutch vocal expressions of emotion, the latter two subject groups were still able to identify the great majority of the ten emotions examined beyond chance expectancy. Since it is very improbable that either the Taiwanese or Japanese subjects had had any prior contact with speakers of Dutch, and since, more generally, their exposure to Westem languages produced by native speakers was very limited, these findings suggest that they have responded to charactenstics of vocal expressions of emotion which are cross-culturally, and perhaps even universally, recognizable

The two emotions which the Taiwanese and Japanese subjects identified most accurately were sadness and anger, a finding which parallels what has been found for the cross-cultural recognition of facially expressed emotions. Vocal expressions of neutral - a category which for practical reasons we have been referring to as an emotion too, but which is usually not considered as such - appeared also to be relatively easy to identify for the two foreign 
groups. On the other hand, joy, which because of the universal meaning of the smile came out as by far the best recognized emotion in cross-cultural research into facial expressions, was the emotion which in the present study lost most of its recognizability. Beier and Zautra (1972), who offered American English expressions of six emotions to groups of American English, Polish, and Japanese subjects, obtained a similar result: While in the group of American English subjects joy was the emotion identified correctly most often, in both the foreign subject groups it yielded the second lowest number of correct responses. On the basis of these findings it could be hypothesized that in contrast with facial expressions, vocal expressions of joy, in addition to cues which are cross-culturally similar, possess important cues which are culture specific.

This hypothesis is made more plausible by the outcomes of the hierarchical cluster analyses and multidimensional scaling which were carried out on the symmetrized confusion matrices. Both techniques yielded results which may be interpreted as indications that for all three subject groups, but most clearly for the Taiwanese and Japanese subjects, confusions among emotions were primarily a function of level of activity. This outcome is consistent with the findings of Davitz (1964c), Davitz and Davitz (1959), Dimitrovsky (1964), and Fairbanks and Pronovost (1939) with respect to American English subjects and with the findings of Albas et al. (1976) with respect to Anglo-Canadian white and Cree-Indian subjects: The confusions found in these studies can also be more easily explained in terms of emotions being similar in level of activity than in terms of their being similar in, for example, evaluative meaning or strength. This finding is also in accordance with the results of our own recognition experiments with children, reported on in Chapters 7 and 8.

Dawes and Kramer's study on vocal expressions of emotion (1966) is the only one in which the confusions are reported to be most readily interpretable in terms of a single evaluative dimension, running from love at the one extreme via grief, indifference (in the middle), and contempt to anger at the other extreme. However, in view of the position of grief next to love, we think that an interpretation in terms of activity is just as plausible, to say the least. As we have demonstrated in Section 4.4.2, there are more cases in which the labeling of a dimension as evaluative is not very convincing.

On the other hand, Uldall (1967), interpreting the results of a judgment study using semantic differential scales, rightly stated the evaluative dimension to be the strongest; It accounted for $50 \%$ of the variance against $20 \%$ for the activity dimension. This outcome cannot be generalized to the judgment of vocal expressions of emotions in general though, since in her experiment resynthesized stimuli were offered of which only the intonation contour was varied. Thus, features that are essential to the transmission of level of activity, such as loudness and tension, remained constant.

Furthermore, it is interesting to note that it has proven easier to find the vocal cues transmitting information on level of activity than those transmit- 
ting information on evaluative meaning or strength. This holds for our own descriptive study reported on in Chapter 4 and for other studies as well (see Section 4.4.2).

There thus seems to be fairly strong evidence that both within and across cultures the dominant dimension along which vocal expressions of emotion are discriminated is the activity one and that other dimensions, such as the evaluative one, are secondary. This would lend support to he hypothesis which was formulated very tentatively by Davitz (1964c) that "characteristics of emotional expressions directly associated with the activity dimension of emotional meaning are ... probably unlearned, while the more subtle cues ... which carry emotional meaning in terms of valence and strength are likely to reflect culturally determined patterns of learning" (p. 111). This might be another difference with facial expressions of emotion, since in the discrimination of those the evaluative dimension has almost invariably emerged as the most prominent one: In eleven out of the thirteen facial studies included in the overview presented by Dittman (1972, p. 68-69), the first dimension found was an evaluative one, whereas in only two was it level of activity. This dominance of the evaluative dimension seems to be independent of whether still photos were offered or live and filmed "moving" expressions.

It thus looks as if the face is more fit to express evaluative meaning, while the voice is better equipped to transmit information on level of activity (for the perceptual and acoustic cues, see the remarks with respect to the characteristics of stress in Section 3.4.4 and those pertaining to the correlates of the activity dimension in Section 4.4.2). It must be admitted that the results of the study conducted by Williams and Sundene (1965), a study which was especially designed to compare dimensions of recognition in the facial, vocal, and combined channels, do not confirm this hypothesis: For all three channels the same three dimensions emerged from a factor analysis of the ratings of each time twelve stimuli on sixty-one scales, the first one, accounting for almost $40 \%$ of the variance, being clearly evaluative in nature since it received high loadings from scales such as pleasurable-painful and pleasantunpleasant. The second dimension, which accounted for about $20 \%$ of the variance, was interpreted in terms of "social control" and the third one, accounting for an additional $1 \%$ of the variance, in terms of activity.

The present study further revealed that in addition to symmetrical confusions the subjects made a great number of asymmetrical confusions. As for the former, there were only two which occurred in all three experiments, namely the one between surprise and joy, and the one between disgust and contempt. Both of these are intuitively plausible, since the members of either pair are fairly similar in level of activity as well as evaluative meaning. Moreover, both have been reported with respect to the recognition of facial expressions also (e.g. Schlossberg, 1941; Thompson \& Meltzer, 1964). In fact, many investigators have considered the similarity in facial appearance 
of disgust and contempt to be so great that they did not offer the two emotions separately for recognition (see, for example, the studies included in Table 1.1 in Izard $(1977$, p. 7$)$ ). Interestingly enough, disgust and contempt were the two emotions which in the present research the speakers also declared having most problems with keeping apart.

As for the asymmetrical confusions, there was only one which was made by all three subject groups. This one exception, namely the much more frequent interpretation of fear as surprise than the interpretation of surprise as fear is, for that matter, a very intriguing asymmetrical confusion, since it has been reported very consistently in cross-cultural research into facial expressions of emotion as well (Boucher \& Carlson, 1980; Ekman, 1973). In the present experiment the fact that in all three experiments the category surprise was generally chosen much more often as a response than the category fear might at least partly be responsible for this result. Of course, the question why certain categories are much more favoured than others also remains to be investigated. 


\section{Integration of findings and suggestions for further research}

\subsection{INTRODUCTION}

This book comprises two series of studies, the first related to the description of emotions (Chapters 3, 4, and 5) and the second to the recognition of, or discrimination among, emotions (Chapters 6, 7, 8, and 9). Both types of studies are based upon (parts of the same material so that it is possible to relate the outcomes of one type to the outcomes of the other. This is a fairly unique situation since in most studies to date only one of the two approaches has been adopted: Attention was either focussed on finding the perceptual and acoustic characteristics of emotional expressions or on assessing the extent to which subjects are able to infer emotional meaning from expressions offered. The relationships between the two domains have rarely been established.

In the first part of this chapter we will combine descriptive and recognition results. First we will consider the question of the cross-cultural similarity of the expressions of discrete emotions; next, we will discuss the nature and role of emotional dimensions; and finally, we will pay attention to the success with which the ten emotions considered in this research can be discriminated from one another, either statistically on the basis of descriptive data or by human subjects. We will not give any detailed information on the methods which have been used to obtain the different results since this would mean repeating what has been extensively described in the preceding chapters. In the second part of this chapter some conlusions will be drawn from this research which are more methodological in nature, and some suggestions will be made for further research.

\subsection{CROSS-CULTURAL SIMILARITY OF DISCRETE EMOTIONS}

In Chapter 3, a partial comparison was made between the perceptual characteristics of vocal expressions of ten discrete emotions as they appeared from our own description for Dutch with those reported for a number of other European languages (data with respect to non-European languages are lacking). The data for shame and interest were too scarce to permit any statement as to possible cross-cultural similarities or differences. The cross- 
cultural findings for the emotions on which more information was available agreed fairly well. For joy and anger there was even almost total agreement. These descriptive data may be compared to the results of our cross-cultural recognition experiments reported on in Chapter 9.

In general, the conclusion of cross-cultural similarity of the expressions of most of the emotions examined was confirmed: Japanese as well as Taiwanese subjects were able to identify the great majority of the ten emotions expressed by Dutch speakers beyond chance expectancy (in fact, there was only one exception, namely the recognition of shame by the Japanese). However, some emotions proved to lose more of their recognizability in the cross-cultural setting than others. A clear example is joy, which was identified correctly by the Dutch subjects $76 \%$ of the time, whereas the percentages for the Taiwanese and Japanese subjects were $24 \%$ and $20 \%$, respectively.

The seemingly contradictory findings for joy - the descriptive data suggest cross-cultural similarity (or even identity), the recognition results cross-cultural differences - may be explained in various ways. First, it must be noted that the descriptive data were derived from studies restricted to a few European languages (Dutch, French, English, German, Hungarian, and Czech). Thus, it could well be that the similarity in characteristic features cannot be generalized to non-European languages such as Taiwanese and Japanese (even the generalization to all European languages is unwarranted). Second, the descriptive data that agreed with one another pertained to global estimates of pitch level, pitch range, loudness, and tempo only. We think it is probable that this description is too rough. We also think that it is incomplete and that joy, in addition to these four parameters, is characterized by more subtle cues which may vary in different cultures or which may be difficult to perceive for someone who is not familiar with the linguistic properties of the language in which the emotions are expressed.

The question of the extent to which vocal expressions of emotion are specific for one culture or a restricted number of related cultures and the extent to which they are culture independent cannot be given a definite answer until both detailed descriptions and recognition results have been collected for a wide variety of different languages.

\subsection{EMOTIONAL DIMENSIONS}

In Chapter 3, a non-dimensional approach was adopted: Perceptual scores on twelve vocal parameters were used to gain insight into the characteristic patterns of discrete emotions. In Chapter 4 , these scores served as a basis for a number of discriminant analyses in order to establish the characteristics of the functions (dimensions) which statistically yielded an optimal separation of the various emotional categories, i.e., attention was focussed on emotional dimensions. The first and most important dimension emerging could easily be labeled as a dimension of activity. This interpretation was based upon the 
way in which the different emotions were positioned along it (shame, neutral, and sadness at one extreme, joy, surprise, and anger at the other, and interest, contempt, disgust, and fear in the middle) as well as upon the fact that the heaviest positive contributions to this function came from laryngeal tension, pitch range, and loudness, and the heaviest negative contribution from harshness. The importance of the parameters related to activity appeared also from the fact that the single most powerful discriminating parameter was loudness. The positions of the emotions along the first dimension emerging from a discriminant analysis based on acoustic measurements also suggested an interpretation in terms of level of activity.

In order to assess whether the vocal characteristics related to the activity dimension may have played an important role in the discrimination among the emotions by human subjects as well, the results of the discriminant analyses may be compared with the outcomes of the multidimensional scaling carried out on the confusion matrices resulting from the Dutch, Taiwanese, and Japanese recognition experiments reported on in Chapter 9. For all three recognition experiments, two solutions were considered: One in which one dimension was extracted and one in which two dimensions were extracted. The dimensions emerging from the one-dimensional solutions had much in common: Along all three roughly three blocks of emotions could be distinguished, namely shame, neutral, and sadness at the one extreme, anger, interest, joy, and surprise at the other, and disgust, fear, and contempt in the middle. The same dimension emerged from the three two-dimensional solutions.

This grouping looks much like the grouping of the emotions along the first dimension emerging from the discriminant analyses carried out on the perceptual ratings and acoustic measurements. On the basis of this outcome it could be hypothesized that level of activity has been one of the main sources of information which the subjects have used to structure the emotional expressions, and that the vocal parameters related to activity, such as loudness, laryngeal tension, and pitch range, are not only important for the separation of emotional expressions in a statistical sense, but also in connection with the classificatory behaviour of human subjects. As was shown in Section 9.4, the conclusion of the primary importance of the activity dimension in the recognition of, or rather the discrimination among, vocal expressions of emotion - both within and across cultures - is confirmed by many research findings to date and lends support to the hypothesis formulated very tentatively by Davitz (1964c) that the vocal parameters reflecting level of activity could be universal. These parameters may be physiologically linked to the raising and lowering of overall tension which accompanies the expression of active and passive emotions, respectively.

The second dimensions emerging from the two dimensional solution of the multidimensional scaling were very different from one another as well as from the second and third dimensions emerging from the discriminant analyses. In none of the cases did we find a dimension which was unequivo- 
cally evaluative in nature. The evaluative dimension has proven more difficult to detect than the activity dimension in other dimensional research into vocal expressions of emotions as well, both with respect to its characteristics and with respect to its role in perception. Perhaps vocal expressions of emotion do not possess any properties which can be arranged on a negative/positive continuum.

That, nevertheless, the emotional expressions do contain information which permits a separation between negative and non-negative emotions, appears from the results of a number of hierarchical cluster analyses. In the minimum method dendrogram resulting from the cluster analysis carried out on the classification matrix yielded by the discriminant analysis based upon the perceptual scores and in the minimum and maximum method dendrograms resulting from the cluster analysis carried out on the recognition data obtained with Dutch adults (see Figures 4.3 and 9.4), the three positive emotions of surprise, interest, and joy were grouped together into one cluster. In both these dendrograms there were, in addition, two negative clusters, an active one comprehending disgust, contempt, and anger and a passive one comprehending fear, sadness, and shame, and a fourth cluster, constituted by neutral. Thus, at least in two cases a non-dimensional approach did suggest a structure in which the emotions were grouped not only according to their level of activity but also according to their evaluative meaning.

However, a separation into groups of negative and positive emotions did not appear to be present in the dendrograms resulting from the cluster analyses carried out on the Japanese and the Taiwanese recognition data and the one carried out on the classification matrix resulting from a discriminant analysis based on acoustic measurements (in fact, the "foreign" dendrograms are quite similar to the "acoustic" dendrograms, see Figures 5.3, 9.5, and 9.6). This outcome suggests that the vocal cues carrying evaluative meaning must be very subtle. In addition, our results could be taken as a confirmation of the second part of the hypothesis formulated by Davitz (1964c) that, in contrast to the characteristics of vocal expressions of emotion directly associated with the activity dimension, the cues which carry, for example, evaluative meaning may be culture dependent.

The dendrograms resulting from the cluster analysis carried out on the recognition data of the Dutch adults served as a basis to gain insight into the classificatory behaviour of Dutch children (age $31 / 2$ to 11 ). Just like the Dutch adults, the Dutch children manifested a strong tendency to make confusions within the cluster of the positive emotions, that of the negative passive emotions, and that of the negative active emotions. Thus, they also appear to be able to make use of cues carrying information not only on level of activity but also on the evaluative meaning of emotions. In those cases in which emotions belonging to different clusters were confused, there appeared to be a weak tendency to confuse emotions similar in level of activity rather than emotions similar in evaluative meaning. This suggests that for the children 
also the activity dimension may have played a more important role than the evaluation dimension, a finding which is consistent with our other results and with what is reported in the literature.

\subsection{RATE OF RECOGNITION}

In several chapters of this study, data have been presented with respect to the extent to which the emotions examined can be distinguished from one another, either statistically on the basis of perceptual ratings (Chapter 4) or acoustic measurements (Chapter 5), or by different groups of human subjects. The relative performance of Dutch adults compared to Dutch children, and that of Dutch adults compared to foreign subjects has been dealt with in Chapters 7 and 8 , and Chapter 9 , respectively. Here we will only compare the percentages correct identification yielded by the perceptually based classification, those yielded by the acoustically based classification, and those attained by Dutch adults.

As for the classification on the basis of perceptual ratings, we take as our point of departure the percentages correct yielded by the discriminant analysis with three functions and five discriminating variables, namely loudness, laryngeal laxness, pitch level, pitch range, and laryngeal tension. The reason for taking the five variable solution, and not one of the higher ordered solutions, is that adding more variables did not really increase the number of utterances correctly classified. The five variable solution yields, in other words, the optimal result that can be accomplished with the minimum amount of perceptual information. As for the classification on the basis of acoustic measurements, we take as our point of departure the discriminant analysis with three functions and seven (i.e., all available) discriminating variables, namely syllables-per-second without pauses (a correlate of tempo), $F_{0}$ median (a correlate of pitch level), $F_{0}$ variation coefficient (a correlate of pitch range), uncorrected $F_{0}$ perturbation (a correlate of harshness), and three spectral slope measures (a composite correlate of laryngeal laxness, laryngeal tension, and loudness).

Since both the perceptually based and the acoustically based classifications pertain to the emotions expressed with twee maanden zwanger and $z 0^{\prime} n$ grote Amerikaanse auto, it was necessary to calculate the percentages correct for the Dutch adults for these two phrases as well (these percentages have not been presented in any of the preceding chapters). In order to facilitate comparison, the percentages correct identification yielded by the three procedures have been put together in Table 10.1, separately for each of the ten emotions and averaged over the ten emotions.

It may be seen from Table 10.1 that the mean percentages correct yielded by the perceptually based discriminant analysis and attained by the Dutch adults are fairly close together. It appears possible, in other words, to obtain an overall correct identification of emotional expressions with an automatic 
Table 10. I Percentages of correct identifications of ten emotions yielded by automatic procedures based on acoustic and perceptual data and attained by Dutch adults

\begin{tabular}{|c|c|c|c|c|c|c|c|c|c|c|c|}
\hline \multirow[t]{2}{*}{ Classification } & \multicolumn{11}{|c|}{ Emotions } \\
\hline & NE & DI & SU & SH & IN & JO & FE & $\mathrm{CO}$ & SA & AN & Mean \\
\hline Acoustically based & 50 & 44 & 62 & 38 & 69 & 31 & 38 & 31 & 19 & 50 & 43 \\
\hline Perceptually based & 94 & 50 & 44 & 62 & 81 & 38 & 75 & 31 & 62 & 75 & 61 \\
\hline By human subjects & 89 & 48 & 69 & 57 & 72 & 76 & 58 & 52 & 72 & 74 & 67 \\
\hline
\end{tabular}

procedure based upon the perceptual ratings for five parameters which is virtually just as good as that which is reached with human subjects. Of course, this is in a situation in which a priori knowledge about the group memberships of the utterances were part of the input to the analysis. The weightings of the variables were based, in other words, on the specific properties of the emotional utterances that were later to be classified. It would be interesting to compare the performance of the human subjects with the performance of a discriminant analysis in which the same functions were used to classify a new set of emotional expressions. This would be a better approximation of what is requested of the human subjects: They also have to apply the "weightings" of variables derived from previous experiences with emotional expressions to a set that they have not heard before.

The acoustically based discriminant analysis is considerably less successful than either the perceptually based analysis or the performance of human subjects. From this it could be inferred that a considerable part of the variance in the acoustic measurements that is not shared with the variance in the perceptual data which they represent cannot be used to separate the emotional utterances.

A comparison of the percentages correct for the individual emotions shows that there are two emotions for which the three procedures do about equally well, namely disgust and, somewhat less convincingly, interest. If the perceptually and acoustically based classifications are compared, it appears that for the other eight emotions the former does usually considerably better than the latter. Exceptions are surprise, for which the opposite is the case, and contempt, for which there is no difference. A comparison of the perceptually based classification results with those obtained with human subjects reveals that there are about as many emotions for which the former does better than the latter as there are emotions for which the opposite is the case. The most conspicuous difference is found in the identification of joy, with a difference of $38 \%$ in favour of the human subjects. Again an indication that joy is characterized by some cues which are very difficult to grasp descriptively. It is our impression that these cues might be spectral in nature.

The above results pertain directly to the nature and structure of vocal expressions of emotion. Now we will present some findings which are interesting from a methodological point of view. 


\subsection{SOME METHODOLOGICAL REMARKS AND SUGGESTIONS FOR FURTHER RESEARCH}

As for the description, we have shown that reliable perceptual judgments may be obtained for many more than the four parameters commonly examined. Although statistically parameters such as tremulousness, creak, and lip spreading did not seem to contribute very much to the separation of the emotions (Chapter 4), we have argued that this may reflect the properties of the type of analysis used rather than their true value. It seems plausible, for example, that, especially in an intra-cultural setting, the auditory effect of lip spreading may be of help to a human listener to distinguish joy from other emotions (this is not to say that we think that lip spreading is always a component of the vocal expression of joy). Moreover, the fact that certain parameters did not seem to be very important for the separation of the Dutch expressions does not necessarily mean that they could not be of relevance for the separation of expressions in other languages. We therefore think that in future research, intra-cultural as well as cross-cultural, the range of parameters described should be extended.

Although we are convinced of the value and feasibility of detailed perceptual descriptions, we think that there are a few conditions the fulfilment of which would greatly enhance the comparability and trustworthiness of perceptual data obtained with different (groups of) raters. A first condition is the standardization of the terminology and method used to describe particular auditory effects. The vocal profile analysis protocol developed by Laver (1981) could serve as a basis in this respect, especially since it is accompanied by a tape on which a number of vocal parameters are demonstrated. However, in its present form, it is specifically aimed at the description of pathological voices. This appears not only from the categories it comprehends but also from the way in which the scales have been set up: Most scales are divided into two parts, one which is to be rated in the case of the normal presence and the other which is to be rated in the case of the abnormal presence of a particular feature. This means that if the protocol is to be used for the description of non-pathological emotional utterances the normal/abnormal distinction should disappear and that the categories which are relevant to pathology only, such as audible nasal escape and diplophonia, should be excluded. In fact, there are a number of other categories which could be excluded as well, because they almost certainly are not relevant to the description of emotional expressions (of course, there is always a small chance that in some exotic culture a particular feature is being used to express emotional or attitudinal meaning).

Moreover, in Laver's protocol heavy emphasis is placed on laryngeal and supralaryngeal settings, prosodic features being less extensively covered (the latter are even completely absent from the demonstration tape). Thus, in order to be able to fully grasp the characteristics of emotional utterances, the protocol will have to be supplemented with categories relative to pitch, 
loudness, and tempo variations. Examples of scales which could be added are tempo variability and amount of emphasis (specific pitch contours that may characterize certain emotional categories are impossible to be rated on scales and have to be described separately). Of the prosodic parameters added, and also of the prosodic parameters already included, clear examples will have to be recorded in order to clarify their meaning.

Obtaining a standardized and comprehensive tool for making perceptual descriptions is very important. It is no guarantee, however, that the ratings resulting from its use are reliable and that ratings obtained with different (groups of) raters are comparable. In other words, data are needed with respect to intra- and intergroup reliabilities. If certain categories, even after repeated attempts, would fail to yield sufficiently high reliability coefficients, these parameters should be discarded from the protocol. Intergroup reliabilities, especially those obtained with groups of raters with varying linguistic backgrounds, are useful to determine to what extent perceptual judgments are language dependent. Insight into this question is not only of theoretical interest but also relevant in connection with the quest for acoustic correlates: If particular judgments would appear to be dependent on the linguistic background of the rater it would, of course, still be possible to develop separate acoustic correlates. The acoustic measures would lose, however, a part of their claimed objectivity and value for cross-cultural comparison.

In our own study on acoustic correlates (Chapter 5), there were only two perceptual parameters that were satisfactorily explained by acoustic measures, namely tempo and pitch level. The correlations between the other acoustic measures and perceptual parameters considered were rather low. The relationships between the acoustic and perceptual domains are clearly in need of further research, preferably with longer stretches of speech than those used in the present study (we are presently tackling this problem ourselves with non-emotional speech samples of minimally 60 seconds). The search for acoustic correlates, especially those of prosodic characteristics, would greatly profit from the results of experiments in which the nature of perceptual judgments was examined by offering (re)synthesized stimuli in which different parameters were systematically varied.

Relevant to future recognition studies are our findings that the number of correct identifications may be affected by response bias and, in the case of a simulation approach, by the verbal material which has been selected as the carrier for the emotional expressions (in our own study there was one phrase which was identified significantly worse than the other three, for no clear reason). Both potentially disturbing factors are in need of further investigation. Of course, in spite of the methodological problems involved, efforts to find ways to study non-simulated emotional expressions should be stimulated. Various content masking techniques, such as random splicing and lowpass filtering, are available to eliminate possibly confounding effects of emotional meaning contained in the verbal channel of communication, retaining different vocal cues transmitting emotional meaning. 


\section{APPENDIX A}

\section{ACOUSTIC DATA FOR TEN EMOTIONS (Chapter 5)}

$\mathrm{F}_{0}$ variation coefficient $(s / m \times 100$, in which $s=$ the standard deviation and $m=$ the mean of the $\mathrm{F}_{0}$ distribution $(N=16)$

\begin{tabular}{lrrrr}
\hline & Mean & $s$ & Minımum & Maximum \\
\hline Neutral & 3498 & 1113 & 155 & 551 \\
Disgust & 5418 & 1935 & 195 & 897 \\
Surprise & 11346 & 3446 & 777 & 1871 \\
Shame & 3932 & 2138 & 81 & 752 \\
Interest & 6492 & 1736 & 306 & 1084 \\
Joy & 6407 & 2270 & 245 & 1155 \\
Fear & 4839 & 2048 & 207 & 1099 \\
Contempt & 4744 & 2045 & 127 & 832 \\
Sadness & 4896 & 3464 & 60 & 1106 \\
Anger & 5414 & 2085 & 182 & 817 \\
\hline
\end{tabular}

Fo median (in $\mathrm{Hz})(N=8)$

\begin{tabular}{llrlllllll}
\hline & Females & \multicolumn{7}{c}{ Males } \\
\cline { 2 - 9 } & Mean & \multicolumn{1}{c}{$s$} & Min & Max & Mean & $s$ & Mın & Max \\
\hline Neutral & 190 & 12 & 178 & 211 & 103 & 7 & 96 & 117 \\
Disgust & 178 & 33 & 151 & 245 & 121 & 22 & 98 & 157 \\
Surprise & 332 & 104 & 178 & 512 & 152 & 53 & 84 & 212 \\
Shame & 188 & 15 & 158 & 205 & 108 & 17 & 92 & 144 \\
Interest & 307 & 42 & 248 & 373 & 176 & 35 & 132 & 212 \\
Joy & 313 & 122 & 191 & 537 & 246 & 64 & 190 & 364 \\
Fear & 312 & 73 & 242 & 456 & 203 & 35 & 154 & 255 \\
Contempt & 206 & 62 & 118 & 284 & 109 & 19 & 78 & 132 \\
Sadness & 247 & 32 & 216 & 309 & 155 & 37 & 100 & 223 \\
Anger & 271 & 40 & 206 & 320 & 222 & 30 & 194 & 276 \\
\hline
\end{tabular}

Number of syllables per second (after subtraction of the pauses) $(N=16)$

\begin{tabular}{lllll}
\hline & Mean & $s$ & Minımum & Maxımum \\
\hline Neutral & 502 & 073 & 364 & 643 \\
Disgust & 280 & 092 & 139 & 455 \\
Surprise & 435 & 138 & 221 & 835 \\
Shame & 408 & 105 & 202 & 609 \\
Interest & 533 & 109 & 334 & 706 \\
Joy & 418 & 102 & 236 & 587 \\
Fear & 506 & 143 & 297 & 818 \\
Contempi & 324 & 101 & 198 & 523 \\
Sadness & 399 & 111 & 173 & 594 \\
Anger & 435 & 113 & 221 & 626 \\
\hline
\end{tabular}


INSTRUCTIONS FOR THE RECOGNITION EXPERIMENT WITH DUTCH ADULTS

(translation from Dutch) (Chapter 6)

If you want to know how someone feels, you can derive this not only from what someone says but also from how he or shesaysit. Thus, the way in which an utterance is spoken contains information about the emotion which the speaker is experiencing. By means of this experiment we want to find out to what extent people, solely on the basis of intonation, are able to determine with which emotion an utterance has been emitted. By the term "intonation" we do not only refer to pitch and changes in pitch, but also to such speech characteristics as tempo and voice quality.

In a moment you are going to hear a number of recordings of women and men producing four phrases (daar is ie weer, twee maanden zwanger, ze is bij de luchtmacht, zo'n grote Amerikaanse auto) with ten different emotions (disgust, surprise, shame, interest, joy, neutral, fear, contempt, sadness, anger), but first we would like to know with which emotion you associate the verbal content of the different phrases. Please indicate your associations below by encircling one emotion for each of the four phrases.

1. Daar is ie weer

2. Twee maanden zwanger

\begin{tabular}{|l|l|l|l|l|}
\hline disgust & shame & joy & fear & sadness \\
\hline surprise & interest & neutral & contempt & anger \\
\hline
\end{tabular}

\begin{tabular}{|l|l|l|l|l|}
\hline disgust & shame & joy & fear & sadness \\
\hline surprise & interest & neutral & contempt & anger \\
\hline
\end{tabular}

3. Ze is bij de luchtmacht

\begin{tabular}{|l|l|l|l|l|}
\hline disgust & shame & joy & fear & sadness \\
\hline surprise & interest & neutral & contempt & anger \\
\hline
\end{tabular}

4. Zo'n grote Amerikaanse auto

\begin{tabular}{|l|l|l|l|l|}
\hline disgust & shame & joy & fear & sadness \\
\hline surprise & interest & neutral & contempt & anger \\
\hline
\end{tabular}


We will now start the recognition experiment. We ask you to listen very carefully to the recordings and determine which emotion the speaker has tried to express. You indicate your choice by encircling one and only one emotion for each utterance on your score sheet (the numbers on the sheet correspond with the numbers preceding the utterances on the tape).

You will first hear six utterance to practice. After you have rated these practice utterances below there will be time for you to ask questions in case something is not clear.

\begin{tabular}{|c|c|c|c|c|c|c|c|c|c|}
\hline disgust & surprise & shame & interest & joy & neutral & fear & contempt & sadness & anger \\
\hline disgust & surprise & shame & interest & joy & neutral & fear & contempt & sadness & anger \\
\hline disgust & surprise & shame & interest & joy & neutral & fear & contempt & sadness & anger \\
\hline disgust & surprise & shame & interest & joy & neutral & fear & contempt & sadness & anger \\
\hline disgust & surprise & shame & interest & joy & neutral & fear & contempt & sadness & anger \\
\hline disgust & surprise & shame & interest & joy & neutral & fear & contempt & sadness & anger \\
\hline
\end{tabular}

We will now continue with the proper set of utterances. There are four blocks of 80 utterances each, alternately produced by female and male speakers. Within the blocks individual speakers, phrases, and emotions have been put in random order. The different emotions do not necessarily occur the same number of times; it is, in other words, possible that some emotions will be presented more frequently than others. After each block you will have a break of ten minutes to relax. The total duration of the experiment is two hours. 
APPENDIX C

COMBINATIONS AND ORDER OF THE ALTERNATIVES IN THE DEVELOPMENTAL RECOGNITION EXPERIMENT (Chapter 7)

\begin{tabular}{lll}
\hline Disgust (1) & Anger (2) & Surprise (3) \\
Joy (2) & Surprise (1) & Shame (3) \\
Contempt (3) & Shame (1) & Fear (2) \\
Disgust )3) & Surprise (2) & Interest (1) \\
Joy (1) & Interest (2) & Fear (3) \\
Sadness (2) & Anger (3) & Fear (1) \\
Contempt (1) & Sadness (3) & Disgust (2) \\
Interest (3) & Sadness (1) & Shame (2) \\
Contempt (2) & Joy (3) & Anger (1) \\
\hline
\end{tabular}

(1) = key

(2) = distractor related to the key

(3) = distractor not related to the key

\section{APPENDIX D}

COMBINATIONS AND ORDER OF THE ALTERNATIVES IN THE RECOGNITION EXPERIMENT WITH TODDLERS (Chapter 8)

\begin{tabular}{lll}
\hline Disgust (1) & Anger (2) & Interest (3) \\
Anger (3) & Interest (1) & Joy (2) \\
Fear (3) & Joy (1) & Interest (2) \\
Sadness (2) & Joy (3) & Fear (1) \\
Fear (2) & Disgust (3) & Sadness (1) \\
Anger (1) & Disgust (2) & Sadness (3) \\
\hline
\end{tabular}

(1) = key

(2) = distractor related to the key

(3) = distractor not related to the key 


\section{References}

Albas, D C., McCluskey, K.W , \& Albas, C A. (1976) Perception of the emotional content of speech: A comparison of two Canadian groups. Journal of Cross-cultural Psychology, 7, 481-490.

Askenfelt, A \& Hammarberg, B. (1981) Speech waveform perturbation analysis revisited. Speech Transmission Laboratory - Quarterly Progress Status Reports, 4, 49-68.

Asendorpf, J. \& Wallbott, H.G. (1979) Masse der Beobachteruebereınstımmung. Eın systematischer Vergleich. Zettschrift fuer Socialpsychologie, 10, 243-252.

Beier, E.C. \& Zautra, A J. (1972) Identification of vocal communication of emotions across cultures. Journal of Consuling and Clinical Psychology, 39, 166

Beldoch, M. (1964) Sensitivity to expression of emotional meaning in three modes of communication. In J.R. Davitz (Ed), The communication of emotional meaning. Greenwood Press, Westport, Ct., 31-42.

Bezooıjen, R. van (1983) Een vergelıjkende stemkwaliteitbeschrıjving van vier groepen Amsterdammers Spektator, 3, 182-192

Blanck, P D , Rosenthal, R., Snodgrass, S.E , DePaulo, B M , \& Zuckerman, M. (1981) Sex differences in eavesdropping on nonverbal cues Developmental changes. Journal of Personality and Social Psychology, 41, 391-396.

Blom, J.G \& Herpt, L W.A. van (1976) The evaluation of jury judgments on pronunciation quality. Proceedings of the Institute of Phonetic Sctences, University of Amsterdam, 31-46.

Bluhme, T (1971) L'identıficatıon de différentes attıtudes émotıonelles par l'ıntonatıon Travaux de l'institut de phonétıque de Strasbourg, 3, 248-260.

Bortz, J (1966) Physıkalısch-akustische Korrelate der vokalen Kommunikation. Arbetten aus dem psychologischen Institut der Universitaet Hamburg, 9.

Boucher, J.D. \& Carlson, GE (1980) Recognition of facial expressions in three cultures. Journal of Cross-cultural Psychology, 11, 263-280

Bouwhuis, D G (1974) The recognition of attitudes in speech. IPO Annual Progress Report, 9, 82-86.

Boves, L (1984)The phonetic basis of perceptual ratings of running speechs. Foris Publications, Dordrecht-Holland/Cinnaminson-U.S.A.

Boves, L. \& Cranen, B (1982) On long-term average spectra and their representation. Proceedings of the Institute of Phonetics, University of Nijmegen, 6, 20-30.

Brown, B L (1980) The detection of emotion in voice qualities In H Giles, W.P Robinson, \& P M. Smith (Eds.) Language. Social psychological perspectives. Pergamom Press, Oxford, 237-245

Buitıng, H.J.A G (1981) Moses: A simple speech editing facility Proceedings of the Instituse of Phonetics, University of Nimegen, 5, 58-63.

Burns, L. \& Beier, G. (1973) Significance of vocal and visual channels in the decoding of emotional meaning. Journal of Communication, 23, 118-130

Constanzo, F S , Markel, N N , \& Constanzo, P R. (1969) Voıce quality profile and perceived emotion Journal of Counseling Psychology, 16, 267-270. 
Cooley, W.W \& Lohnes, P.R. (1971) Multivariate data analysis Wiley, New York. Crystal, D. (1969) Prosodic systems and intonation in Enghsh. Cambridge University Press, Cambridge.

Crystal, D. (1975) The English tone of volce. Arnold, London.

Crystal, D. \& Quirk, R. (1964) Systems of prosodic and paralinguistıc features in English. Mouton, The Hague.

Darwin, C. (1872) The expression of the emotions in man and animals. John Murray, London (Reprinted, University of Chicago, Chicago, 1965).

Dashiell, J.F. (1927) A new method of measuring reaction to facial expressions of emotion. Psychological Bulletin, 24, 174-175.

Davitz, J R. (1964a) A review of research concerned with facial and vocal expressions of emotions. In J R. Davitz (Ed.), The communication of emotional meaning. Greenwood Press, Westport, Ct., 13-31.

Davitz, J.R. (1964b) Personality, perceptual, and cognitive correlates of emotional sensitivity. In J.R. Davitz (Ed.), The communication of emotianal meaning. Greenwood Press, Westport, Ct., 57-68.

Davitz, J.R. (1964c) Auditory correlates of vocal expressions of emotional meaning. In J.R. Davitz (Ed.), The communication of emotional meaning. Greenwood Press, Westport, Ct., 101-112.

Davitz, J.R. (1964d) The communication of emotional meaning. Greenwood Press, Westport, $\mathrm{Ct}$..

Davitz, J.R. \& Davitz, L.J. (1959) The communication of feelings by content-free speech. Journal of Communication, 9, 6-13.

Dawes, R.M. \& Kramer, E. (1966) A proximity analysis of vocally expressed emotion. Perceptual and Motor Skills, 22, 571-574.

Dickey, E.C. \& Knower, F.H. (1941) A note on some ethnological differences in recognition of simulated expressions of the emotions. American Journal of Socio$\log y, 47,190-193$.

Dimitrovsky, L. (1964) The ability to identify the emotional meaning of vocal expressions at successive age levels In J.R Davitz (Ed.), The communication of emorional meaning. Greenwood Press, Westport, Ct., 69-86.

Dittman, A.T. (1972) Interpersonal messages of communication. Springer Publishıng Company, New York.

Duncan, S.D. \& Rosenthal, R. (1968) Vocal emphasis in expenmenters' instruction reading as unıntended determınant of subjects' responses. Language and Speech, 2, 20-26.

Dusenbury, D. \& Knower, F H. (1938) Experımental studies of the symbolism of action and voice II: A study of the specificity of meaning in abstract tonal symbols. Quarterly Journal of Speech, 25, 67-75.

Ekman, P. (1973) Cross-cultural studies of facial expression. In P. Ekman (Ed.), Darwin and facial expressions: A century of research in review. Academic Press, New York, 169-222.

Ekman, P. \& Friesen, W.V. (1971) Constants across cultures in the face and emotion. Journal of Personality and Social Psychology, 17, 124-129.

Ekman, P. \& Friesen, W.V. (1978) The Facial Action Coding System. A technique for the measurement of facial movement. Consulting Psychologists Press, Palo Alto, Calıf..

Ekman, P., Friesen, W.V., \& Ellsworth, P. (1972) Emotion in the human face. Pergamom Press, New York.

Esling, J.H. (1978) Voice quality in Edinburgh. A sociolinguistic and phonetsc study. Doctoral dissertation, University of Edınburgh.

Faırbanks, G \& Hoaglın, L.W. (1941) An experımental study of the durational characteristics of the voice during the expression of emotion. Speech Monographs, 8, 85-90. 
Faırbanks, G. \& Pronovost, W. (1939) An experimental study of the pitch characteristics of the voice dunng the expression of emotion. Speech Monographs, 6, 87-104.

Fant, G. (1979) Glottal source and excitation analysis. STL-QPSR 1, Speech Transmission Laboratory, KTH, Stockholm, 85-105.

Fechner, E.H. (1978) Children's understanding of the nonverbal communication of emotion in the visual, vocal and gestural. Dissertation Abstracts International, 39. 2568-2569.

Fenster, A. (1967) Vocal communication of emotional meaning among adults and children. Dissertanion Abstracts International, 28, 1694-1695.

Fenster, C.A., Blake, L.K , \& Goldstein, A.M. (1977) Accuracy of vocal emotional communications among children and adults and the power of negative emotions. Journal of Communication Disorders, 10, 301-314.

Fenster, C.A. \& Goldstein, A.M (1971) The emotional world of children vis à vis the emotional world of adults: An examination of vocal communication. Journal of Communication, 21, 353-362.

Ferguson, G.A (1976) Statıstical analysis in psychology \& education. McGraw-Hıll Kogakusha, Tokyo.

Fónagy, I. \& Magdics, K. (1963) Emotional patterns in intonation and music. Phonetlca, 16, 293-326.

Frıda, N.H. (1970) Emotion and recognition of emotion. In M.L. Arnold (Ed.) Feelings and emorions: The Loyola Symposium. Academic Press, New York, London, 241-250.

Gates, G.S (1923) An expenmental study of the growth of social perception. Journal of Educational Psychology, 14, 449-461.

Gates, G.S. (1927) The role of the auditory element in the interpretation of emotions. American Psychological Association, 24, 175.

Gilbert, D C. (1969) The young child's awareness of affect Child Development, 40, 629-640.

Gitter, A.G., Mostofsky, D I., \& Quincy, A.J. (1971) Race and sex differences in the child's perception of emotion. Child Development, 42, 2071-2075.

Green, R.S. \& Cliff, N. (1975) Multıdımensıonal comparısons of structures of vocally and facially expressed emotion. Perception and Psychophysics, 17, 429-438

Hall, J A (1978) Gender effects in decoding nonverbal cues. Psychological Bulletin, $85,845-857$.

Hammarberg, B., Frıtzell, B., Gauffin, J., Sundberg, J , \& Wedın, L. (1980) Perceptual and acoustic correlates of abnormal voice qualities Acta Otolaryngal, 441-451.

Hart J. 't. (1981) Differential sensitivity to pitch distance, particularly in speech. Journal of the Acoustical Society of America, 69, 811-821.

Heıke, G. (1969) Sprachliche Kommunikation und linguistische Analyse Carl Winter Unıversitaetsverlag, Heidelberg

Honikman, B (1964) Artıculatory settıngs In D. Abercrombie, D.B. Fry, P.A.D. MacCarthy, N C Scott, \& J.L.M. Trim (Eds.), In honour of Daniel Jones. Longmans, London, 73-84.

Hornstein, M.G (1967) Accuracy of emotional communication and interpersonal compatibility. Journal of Personality, 35, 20-30

Huttar, G.L. (1968) Relations between prosodic varıables and emotions in normal American English utterances Journal of Speech and Hearing Research, 11, 481-487. Izard, C.E. (1971) The face of emotion Appleton-Century-Crofts, New York.

Izard, C.E (1977) Human emotions. Plenum Press, New York.

Johnson, S.C (1967) Hierarchical clustenng schemes. Psychometrica, 32, 241-254. Kaiser, L. (1962) Communication of affects by single vowels. Synthese, 14, 300-319. Kırk, R. (1968) Experimental design' Procedures for the behavioral sciences. Brooks, Belmont, Calif .

Klecka, W.R. (1975) Dıscrımınant analysıs In N.H. Nie, C.H. Hull, J.G. Jenkıns, K. 
Steınbrenner, \& D H. Bent (Eds), SPSS · Statistical Package for the Social Sciences (2nd ed ) McGraw-Hıll, New York, 434-467

Kleın, W., Plomp, R., \& Pols, L.C.W. (1970) Vowel spectra, vowel spaces, and vowel identification. Journal of the Acoustical Society of America, 48, 999-1009

Knower, F H (1941) Analysis of some experimental variations of simulated vocal expressions of the emotions. Journal of Social Psychology, 14, 369-372.

Kramer E. (1964) Elımınation of verbal cues in judgements of emotion from voice Journal of Abnormal and Social Psychology, 68, 390-396.

Labarbera, J D , Izard, C E., Vietze, P., \& Parısı, S A. (1976) Four- and six-monthold infants' visual responses to joy, anger and neutral expressions. Child Development, 47, 535-538

Laver, J. (1968) Voice quality and indexical information. Britısh Journal of Disorders and Communication, 3, 43-54.

Laver, J. (1975) Individual features in voice qualty. Unpublished doctoral dissertation, University of Edinburgh.

Laver, J. (1979) The description of voice quality in general phonetic theory. Work in progress, Dept of Linguistics, University of Edinburgh, 12, 30-52

Laver, J. (1980) The phonetic description of voice quality Cambridge University Press, Cambridge

Laver, J. (1981) Users' manual for vocal profile analysis protocol: A perceptual guide. Unpublished paper, University of Edinburgh.

Laver, J \& Hanson, R. (1981) Describıng the normal voice. In J.K Darby (Ed.), Speech evaluation in psychiatry. Grune \& Stratton, New York, 51-78

Laver, J. \& Trudgill, P (1979) Phonetic and linguistic markers in speech In K R. Scherer and H Giles (Eds.), Social markers in speech Cambridge Unıversity Press, Cambridge, 1-32.

Laver, J., Wirz, S., MacKenzie, J, \& Hıller, S (1981) A perceptual protocol for the analysis of vocal profiles. Work in progress, Dept of Linguistics, University of Edinburgh, 14, 139-155

Levitt, E.A. (1964) The relationship between abılitıes to express emotional meanıngs vocally and facially In $J$. Davitz (Ed ), The communication of emottonal meaning. Greenwood Press, Westport, Ct., 87-100

Levy, P K (1964) The ability to express and perceive vocal communications of feeling. In J R. Davitz (Ed.), The communication of emotional meaning Greenwood Press, Westport, Ct., 43-55

Lieberman, P \& Michaels, S B (1962) Some aspects of fundamental frequency and envelope amplitude as related to the emotional content of speech. In D Bolinger (Ed), Intonation Penguin Books, Harmondsworth, 235-249

Maitland, G.E. (1978) The perception of facial and vocal expressions of emotions by learning disabled, emotionally disturbed, and normal children. Dissertation Abstracts International, 38, 5396

Markel, N N. (1965) The relıabilıty of coding paralanguage: Pitch, loudness, and tempo. Journal of Verbal Learning and Verbal Behavior, 4, 306-308

Markel, N.N., Beın, M.F., \& Phıllıs, J A. (1973) The relatıonshıp between words and tone-of-voice Language and Speech, 16, 15-21.

McCluskey, K W , Albas, D.C., Niemı, R R., Cuevas, C , \& Ferrer, C A (1975) Cross-cultural differences in the perception of the emotional content of speech: $A$ study of the development of sensitivity in Canadian and Mexican children. Developmental Psychology, 11, 551-555

McCluskey, K.W., Niemı, R.R., \& Albas, D C. (1978) Vocal communication of emotional meaning among normal and disturbed children. Journal of Special Education, 12, 443-449.

Odom, R D \& Lemond, C.M (1972) Developmental differences in the perception and production of facial expressions. Child Development, 43, 359-369 
Osgood, C E , Sucl, G.J., \& Tannenbaum, P.H (1957) The measurement of meaning. University of Illınois Press, Urbana.

Osser, H A. (1964) A "distınctive features" analysis of the vocal communication of emotion. Dissertation Abstracts, 25, 3708.

Pfaff, P.L. (1954) An experimental study of the communication of feeling without contextual material. Speech Monographs, 21, 155-156.

Plaıkner, D. (1970) Die Veraenderungen der menschlichen Stimme unter dem Einfluss psychischer Belastung Unpublished doctoral dissertation, Philosophische Fakultaet der Universitaet Innsbruck

Plomp, R. (1976) Aspects of tone sensation. A psychophysical study. Academic Press, London.

Plutchik, R. (1965) What is an emotion? Journal of Psychology, 61, 295-303.

Plutchik, R. (1980) Emotion: A psychoevolutionary synthesis. Harper \& Row, New York.

Pollack, I., Rubenstein, H., \& Horowitz, A. (1960) Communication of verbal modes of expression. Language and Speech, 3, 121-130.

Pols, L.C.W (1977) Spectral analysis and identification of Dutch vowels in monosyllabic words. Academic Press, Amsterdam.

Reid, D W \& Ware, E E. (1971) A factorial study of judgements of vocally expressed emotion and meaning. Journal of Social Psychology, 84, 161-162.

Rogers, P.L., Scherer, K.R., \& Rosenthal, R (1971) Content filtermg human speech: A simple electronic system. Behavioral Research Methods and Instrumentation, 3 , 16-18.

Rosenthal, R. \& DePaulo, B.M. (1979) Sex differences in eavesdropping on nonverbal cues. Journal of Personality and Social Psychology, 37, 237-285.

Ross, M., Duffy, R.J., Cooker, H S , \& Sargeant, R.L (1973) Contribution of the lower audible frequencies to the recognition of emotions. American Annals of the Deaf, 118, 37-42.

Rossum, N. van (1981) Parallel power density measurement system. Proceedings of the Institute of Phonetics, University of Nijmegen, 5, 142-159.

Rossum, N. van \& Boves, L. (1978) An analog pitch-period extractor. Proceedings of the Institute of Phonetics, University of Nijmegen, 2, 1-17

Scherer, K R. (1971) Randomized splicing. A note on a simple technique for maskıng speech content. Journal of Research in Personality, 5, 155-159.

Scherer, K R (1974) Voice quality analysis of American and German speakers. Journal of Psycholingutstic Research, 3, 281-290.

Scherer, K R. (1978) Personality inference from volce quality: The loud volce of extraversion European Journal of Social Psychology, 8, 467-487.

Scherer, K.R. (1979) Nonlinguistic vocal indicators of emotion and psychopathology. In C.E. Izard (Ed ), Emotions in personaluy and psychopatbology Plenum Press, New York, 495-529.

Scherer, K R (1981a) Vocal indicators of stress. In J.K. Darby (Ed ), Speech evaluatuon in psychiatry Grune \& Stratton, New York, 171-187.

Scherer, K R (1981b) Speech and emotional states In J K. Darby (Ed.), Speech evaluation in psychiatry. Grune \& Stratton, New York, 189-220.

Scherer, K.R (1982) Methods of research on vocal communication: Paradigms and parameters In K R Scherer \& P. Ekman (Eds.), Handbook of methods in nonverbal behavior research. Cambridge University Press, Cambridge, 136-198

Scherer, K R., Koıvumakı, J., \& Rosenthal, R (1972) Minımal cues in the vocal communication of affect: Judging emotions from content-masked speech. Journal of Psycholinguistic Research, 1, 269-285.

Scherer, K R. \& Oshınsky, J S (1977) Cue utılization in emotion attribution from auditory stımulı Motivation and Emotion, 1, 331-346.

Schlosberg, H (1941) A scale for the judgment of facial expressions. Journal of Expertmental Psychology, 29, 497-510. 
Schlosberg, H (1954) Three dimensions of emotion Psychological Review, 61, 81-88.

Sedlácek. K. \& Sychra, A (1963) Die Melodie als Faktor des emotıonellen Ausdrucks. Folia Phoniatrica, 15, 89-98.

Skınner, E.R (1935) A calıbrated recording and analysis of the pitch, force and quality of vocal tones expressing happiness and sadness and a determination of the pitch and force of the subjectıve concepts of ordınary, soft and loud tones. Speech Monographs, 2, 81-137

Soskın, W \& Kauffman, P. (1961) Judgment of emotion in wordfree voice samples. Journal of Communtcation, 11, 73-80

Strongman, K.T (1978) The psychology of emonion (2nd ed) John Wiley \& Sons, Chichester, New York.

Tartter, V.C. (1980) Happy talk: Perceptual and acoustic effects of smiling on speech. Perception and Psychophysics, 27, 2427.

Theyssen, $M$ (1973) Overdracht van attitudes in spraak Verslagen van de voorjaarsvergadering van de Nederlandse Vereniging voor Fonetısche Wetenschappen, 21-49.

Thompson, D F. \& Meltzer, L. (1964) Communication of emotional content by facial expression. Journal of Abnormal and Social Psychology, 68, 129-135.

Titze, I (1980) Comments on the myoelastic-aerodynamic theory of phonation. Journal of Speech and Hearing Research, 23, 495-510.

Tomkıns, S S. (1962) Consciousness, imagery and affect. Vol 1. Springer, New York.

Trager, G.L. (1958) Paralanguage: A first approximation. Studies in Linguistics, 13, $1-12$.

Trudgill, P (1974) The social differennation of English in Norwich. Cambridge Unıversity Press, Cambridge.

Turner, J le B. (1964) Schizophrenics as judges of vocal expressions of emotional meanıng. In J R Davitz (Ed.), The communication of emotional meaning. Greenwood Press, Westport, Ct., 129-142.

Uldall, E E (1967) Attitudinal meanings conveyed by intonation contours. Language and Speech, 3, 223-234.

Wagenaar, W A. \& Padmos, P. (1971) Quantitative interpretation of stress in Kruskal's multıdımensıonal scalıng technıque. Britısh Journal of Mothematical and Statistical Psychology, 24, 101-110

Wendahl, R.W (1963) Laryngeal analog synthesis of harsh voice quality. Folia Phoniatrica, 15, 241-250

Wiggers, M (1977) De ontwikkeling van herkenning van emoties vanaf gezichtsexpressies. Unpublished M.A. thesis, Department of Psychology, University of Nijmegen.

Williams, C E. \& Stevens, K N. (1972) Emotion and speech' Some acoustical correlates. Journal of the Acoustical Society of America, 52, 1238-1250

Willıams, C E \& Stevens, K.N. (1981) Vocal correlates of emotional states. In J.K. Darby (Ed), Speech evaluation in psychiatry. Grune \& Stratton, New York, 221-240.

Wıllıams, F. \& Sundene, B (1965) Dimensions of recognition: Visual vs. vocal expression of emotion. Audiovisual Communication Review, 13, 44-52.

Winer, B.J. (1971) Siatısitical principles in experimental design (2nd ed.). McGrawHill Kogakusha, Tokyo

Wolf, G, Gorskı, R., \& Peters, S. (1972) Acquaintance and accuracy of vocal communication of emotions Journal of Communication, 22, 300-305.

Young-Browne, G , Rosenfeld, H.M., \& Horowitz, F.D. (1977) Infant discrimınation of facial expressions. Child Development, 48, 555-562

Zuckerman, M., Hall, J.A , DeFrank, R S , Rosenthal, R (1976) Encoding and decoding of spontaneous and posed facial expressions. Journal of Personality and Social Psychology, 34, 966-977

Zuckerman, M , Lipets, M S , Koıvumakı, J.H , \& Rosenthal, R. (1975) Encoding and decoding nonverbal cues of emotion Journal of Personality and Social Psychology, 32, 1068-1076 


\section{Samenvatting}

In dit proefschrift worden de kenmerken van vocale expressies van emotie in het Nederlands onderzocht en hun herkenbaarheid als een functie van sekse, leeftijd en cultuur. De kenmerken worden voornamelijk in perceptieve termen beschreven, maar er wordt ook aandacht besteed aan de vraag in hoeverre perceptieve scores door akoestische maten kunnen worden vertegenwoordigd. De herkenbaarheid wordt bepaald voor Nederlandse mannen en vrouwen, voor Nederlandse jongens en meisjes, variërend in leeftijd van 3 tot 11 jaar, en voor volwassenen uit Taiwan en Japan. De beschrijvings- en herkenningsgegevens worden aan elkaar gerelateerd.

In Hoofdstuk 1 worden de twee inhoudelijke componenten van het onderzoek, de beschrijvings- en herkenningsexperimenten, ingeleid. Tevens wordt kort ingegaan op de in het onderzoek gecombineerde categoriale en dimensionale benadering van emotionele expressies. Tenslotte wordt de structuur van het boek gegeven.

Hoofdstuk 2 bevat een beschrijving van het spraakmateriaal waarop het onderzoek is gebaseerd, namelijk de gesimuleerde expressies van tien emoties (neutraal, walging, verrassing, schaamte, belangstelling, blijdschap, angst, minachting, verdriet en boosheid) geproduceerd door vier mannen en vier vrouwen met vier zinnen (daar is ie weer, twee maanden zwanger, ze is bij de luchtmacht en zo'n grote Amerikaanse auto). Speciale aandacht wordt besteed aan het probleem van de scheiding van het verbale en vocale communicatiekanaal en aan de argumenten die tot de beslissing hebben geleid om in het hier gepresenteerde onderzoek gesimuleerde expressies te gebruiken in plaats van spontane.

In Hoofdstuk 3 wordt een studie gepresenteerd waarin de helft van het materiaal door zes personen is gescoord op dertien schalen, en wel met betrekking tot lipronding, lipspreiding, laryngale spanning, laryngale ontspanning, "creak" (= krakerigheid), "tremulousness" (= trillerigheid), "whisper" (= heesheid), "harshness" (= schorheid), toonhoogteniveau, toonhoogterange, luidheid, tempo en precisie van articulatie.

Nadat was vastgesteld dat voor alle parameters de betrouwbaarheid van de scores hoog was, zijn op de scores een aantal variantie-analyses en a 
posteriori contrasttoetsen uitgevoerd. De uitkomsten wezen erop dat alle parameters behalve lipronding relevant kunnen zijn voor het onderscheiden van de bestudeerde emoties. Dit is des te aannemelijker aangezien de correlaties tussen de scores op de verschillende schalen niet erg hoog waren.

De opvallendste perceptieve kenmerken van de verschillende emoties zijn ontspanning en een laag toonhoogteniveau voor neutraal; schorheid, een laag toonhoogteniveau en een langzaam tempo voor walging; een grote toonhoogterange voor verrassing; ontspanning, heesheid, een kleine toonhoogterange en een zachte stem voor schaamte; ontspanning en een grote toonhoogterange voor belangstelling; lipspreiding, schorheid, een hoog toonhoogteniveau en een luide stem voor blijdschap; trillerigheid en heesheid voor angst; een langzaam tempo voor minachting; krakerigheid, trillerigheid, een kleine toonhoogterange en onpreciese articulatie voor verdriet; en spanning, schorheid, en een luide stem voor boosheid.

Aangezien alleen toonhoogteniveau, toonhoogterange, tempo en luidheid en slechts enkele emoties in eerder onderzoek min of meer systematisch zijn bestudeerd, was er slechts een gedeeltelijke vergelijking mogelijk tussen de vocale eigenschappen van discrete emoties zoals die in het onderhavige onderzoek voor het Nederlands zijn gevonden en de kenmerken die voor andere talen zijn gerapporteerd. Het ziet ernaar uit dat in ieder geval een aantal emoties, waaronder blijdschap en boosheid, met betrekking tot de vier parameters in verschillende talen op analoge wijze worden gerealiseerd.

Hoofdstuk 4 heeft betrekking op een studie waarin de scores die in Hoofdstuk 3 zijn beschreven als input zijn gebruikt voor een aantal discriminantanalyses teneinde inzicht te krijgen in het relatieve belang en de specifieke bijdragen van de verschillende perceptieve parameters voor de statistische scheiding van de tien bestudeerde emoties. Zowel de volgorde waarin de variabelen zijn opgenomen in een aantal step-wise analyses als de gewichten van de variabelen in de drie functies die in de oplossing met twaalf variabelen zijn geëxtraheerd, wijzen erop dat de scheiding van de emoties voornamelijk teweeg wordt gebracht door luidheid, ontspanning, toonhoogteniveau, toonhoogterange, spanning en schorheid. Met luidheid als de enige voorspeller werd al $32 \%$ van de emotionele expressies aan de juiste categorie toegekend; de analyse met de vier krachtigste variabelen leverde een percentage correcte classificatie van $58 \%$ op, hetgeen slechts $3 \%$ minder is dan met de volledige set van twaalf variabelen werd bereikt. Hoewel tempo, krakerigheid, trillerigheid, lipspreiding, precisie van articulatie en heesheid niet veel aan de classificatie leken bij te dragen, hebben wij gesteld dat dit niet noodzakelijkerwijs betekent dat deze parameters niet van belang zijn voor de karakterisering van emotionele expressies, maar veeleer dat discriminantanalyse er niet in slaagt hun rol aan het licht te brengen.

De drie functies die in de oplossing met twaalf variabelen zijn geëxtraheerd, lijken allemaal iets met activiteit te maken te hebben. Het blijkt over het algemeen erg moeilijk te zijn in vocale expressies van emotie een evaluatieve dimensie aan de oppervlakte te brengen. 
Een hierarchische clusteranalyse die werd uitgevoerd op basis van de gesymmetriseerde classificatieresultaten van de oplossing met twaalf variabelen resulteerde, ten minste by toepassing van de minımummethode, in groepen emoties die we intuitief erg aannemelıjk vonden: neutraal werd apart gegroepeerd, verrassing, blijdschap, en belangstelling vormden samen een groep; walging, minachtıng, en boosheid vormden samen een groep; en angst, verdriet, en schaamte vormden samen een groep.

In Hoofdstuk 5 wordt een poging beschreven om (combinatıes van) akoestısche correlaten te vinden voor zeven van de perceptieve parameters die in Hoofdstuk 3 zıjn genoemd, namelıjk tempo, toonhoogtenıveau, toonhoogterange, schorheid, luidheid, (laryngale) spanning en (laryngale) ontspanning. Voor elke parameter is een beperkt aantal plausibele akoestische maten bekeken.

Enkelvoudige en multıple regressie liet zien dat waargenomen tempo het beste voorspeld kan worden door het aantal lettergrepen per seconde berekend na de pauzes te hebben afgetrokken, waargenomen toonhoogteniveau door de mediaan van de $\mathrm{F}_{0}$-distributie, en waargenomen toonhoogterange door het quotient van de standaarddeviatie en het gemiddelde van de $\mathrm{F}_{0}$-distributie De andere vier perceptieve parameters hebben meer dan een voorspeller: waargenomen schorheid wordt voorspeld door (ongecorrigeerde) $F_{0}$-perturbatie, twee spectrale hellingmaten en de duurmaat, waargenomen luidheid door twee hellıngmaten, waargenomen spanning door de $\mathrm{F}_{0}$-mediaan, twee hellıngmaten en (ongecorrigeerde) $F_{0}$-perturbatie; en waargenomen ontspannıng door twee hellingmaten. Tempo en toonhoogteniveau konden het gemakkelıjkst door akoestısche maten worden gerepresenteerd; ontspanning en schorheid het moellıkst, en toonhoogterange, luidheld en spanning namen een tussenpositie in Met de zeven meest geslaagde akoestische maten als discrimınerende varıabelen in een discrimınantanalyse werd $43 \%$ van de emotionele uitıngen correct geclassificeerd Ook uit deze analyse kwam een actıviteıtsdımensie naar voren.

In Hoofdstuk 6 wordt vastgesteld hoe goed de verschillende emoties door Nederlandse volwassenen kunnen worden herkend en in hoeverre de herkennıng wordt beınvloed door het geslacht van de luisteraars, het geslacht van de sprekers en de zinnen waarmee de uitıngen zijn geproduceerd. Dit is gebeurd door middel van een gedwongen-keuze-experiment waarın vierentwintig mannen en evenveel vrouwen op de stımulı reageerden door telkens uit tien emotıelabels de best passende te kiezen.

Hoewel alle emoties boven kans werden herkend, bleek de herkenning van sommige categorieen, zoals neutraal, boosheid en blijdschap, aanzienlijk makkelıjker dan die van andere, zoals walging, minachtıng en schaamte. De relatieve herkenbaarheid van de emoties zoals die uit het onderhavige onderzoek naar voren kwam, bleek weinıg overeenkomsten te vertonen met de volgordes die in andere studies zıjn gerapporteerd Dit gebrek aan overeen- 
stemming kan een gevolg zijn van verschillen in de opzet van de experimenten, maar het kan ook echte fluctuaties in de herkenbaarheid van de vergeleken emoties weerspiegelen.

Er was geen significant verschil tussen de mannelijke en vrouwelijke sprekers wat betreft de duidelijkheid waarmee ze de verschillende emoties realiseerden en er was ook geen significant verschil tussen de mannelijke en vrouwelijke luisteraars wat betreft de juistheid waarmee ze de verschillende emoties identificeerden. Beide uitkomsten zijn in overeenstemming met de meeste gegevens die eerder onderzoek heeft opgeleverd.

Tenslotte bleek ook dat gemiddeld genomen de emoties aanzienlijk minder vaak correct werden herkend wanneer ze werden geproduceerd met daar is ie weer dan wanneer ze met een van de andere drie zinnen werden uitgedrukt. Het is niet duidelijk waardoor dit afwijkende gedrag is veroorzaakt. Het lijkt in ieder geval niet te liggen aan het feit dat de verbale inhoud van daar is ie weer minder neutraal zou zijn of meer in de richting van een bepaalde emotie zou gaan.

De studie waarover in Hoofdstuk 7 wordt gerapporteerd, heeft betrekking op de herkenning van emoties door Nederlandse kinderen. Centrale vragen in deze studie zijn hoe de herkenning zich vanaf het vijfde levensjaar ontwikkelt, of er in dit opzicht een verschil is tussen jongens en meisjes, of de herkenning afhangt van de manier waarop de proefpersonen gevraagd wordt op de stimuli te reageren en of de fouten die door kinderen worden gemaakt, lijken op de fouten van volwassenen. Er werden expressies aangeboden van alle tien de emoties behalve neutraal. De proefpersonen (vijf jongens en vijf meisjes van 5, 7, 9 en 11 jaar en een controlegroep van vijf volwassen mannen en vijf volwassen vrouwen) reageerden op de stimuli door telkens te kiezen uit drie tekeningetjes waarop verschillende emoties stonden afgebeeld ("de tekeningtoets") of door te kiezen uit drie emotielabels ("de labeltoets"). Alle vijf de leeftijdsgroepen namen deel aan de tekeningtoets; de labeltoets werd alleen bij de drie oudste leeftijdsgroepen afgenomen.

Het aantal correcte identificaties bleek met het oplopen van de leeftijd gestadig toe te nemen. Deze toename bleek voor een groot deel het gevolg te zijn van het steeds beter kunnen herkennen van verrassing, schaamte, belangstelling en - in de tekeningtoets - minachting. Met betrekking tot de andere emoties deed de oudste groep het nauwelijks beter dan de jongste.

Er was geen significant verschil tussen de scores van de jongens en die van de meisjes en ook niet tussen de scores voor de twee toetsen. Waarschijnlijk zouden de scores voor de twee toetsen wel hebben verschild - ten voordele van de tekeningtoets - als het relatieve gedrag van de twee jongste groepen in de beschouwing was betrokken.

Tenslotte kwam ook nog naar voren dat kinderen hezelfde soort fouten maken als volwassenen: beiden hebben de neiging om verwarringen te laten plaatsvinden binnen de cluster van verrassing, belangstelling en blijdschap, die van schaamte, angst en verdriet en die van walging, minachting en 
boosheid. Als emoties uit verschillende clusters met elkaar worden verward, dan lijkt aan overeenkomst in mate van activiteit een wat $\mathrm{zwaarder}$ gewicht te worden toegekend dan aan overeenkomst in evaluatieve betekenis.

In Hoofdstuk 8 wordt een soortgelijk herkenningsexperiment met drie-eneen-half jaar oude kinderen (vijf jongens en vijf meisjes) beschreven. Er werden expressies aangeboden van zes emoties, namelijk walging, belangstelling, blijdschap, angst, verdriet en boosheid.

Als groep bleken de kinderen alle zes de emoties boven kans te kunnen identificeren. Dit is de laagste leeftijd die tot nu toe in verband met de herkenning van vocale expressies van emotie is gevonden. Er was geen significant verschil tussen het aantal correcte scores voor jongens en dat voor meisjes. Tenslotte bracht deze studie aan het licht dat de kinderen sterk geneigd waren om blijdschap en belangstelling met elkaar te verwarren, walging en boosheid, en verdriet en angst. Wanneer er andere verwarringen werden gemaakt, werden emoties die overeenkwamen in mate van activiteit eerder verward dan emoties die overeenkwamen wat betreft hun evaluatieve betekenis.

In Hoofdstuk 9 wordt er een vergelijking getrokken tussen de herkenningsscores van volwassenen uit Nederland, Taiwan en Japan. De Nederlandse groep bestond uit vierentwintig vrouwen en vierentwintig mannen (zie Hoofdstuk 6), de Taiwanese groep uit twintig mannen en twintig vrouwen, en de Japanse groep uit eenenveertig mannen. De proefpersonen reageerden op de stimuli door telkens uit tien emotielabels de best passende te kiezen.

Hoewel de Nederlandse proefpersonen het significant beter deden dan de buitenlandse proefpersonen, bleken de laatste toch nog in staat te zijn om bijna alle emoties boven kans correct te identificeren. Van alle emoties behielden boosheid, verdriet en neutraal in de cross-culturele setting hun herkenbaarheid het best en blijdschap het slechtst. De laatste uitkomst zou erop kunnen wijzen dat, in tegenstelling tot de kenmerken van gezichtsuitdrukkingen van blijdschap, die van vocale expressies van blijdschap voor een belangrijk deel cultuurspecifiek zijn.

De eerste dimensie waarlangs de uitingen werden gediscrimineerd was een activiteitsdimensie. Deze uitkomst komt overeen met het merendeel van de eerder gerapporteerde resultaten met betrekking tot de dimensionaliteit van vocale expressies van emotie. Het wijkt echter opnieuw af van wat de meeste onderzoekingen naar gezichtsuitdrukkingen hebben opgeleverd, namelijk een eerste evaluatieve dimensie.

Tenslotte kwam uit het onderzoek naar voren dat sommige responsiecategorieën veel vaker worden gekozen dan andere en dat veel verwarringen asymmetrisch zijn.

In Hoofdstuk 10 worden de descriptieve gegevens die zijn verkregen in de Hoofdstukken 3, 4 en 5 gerelateerd aan de herkenningsresultaten die zijn 
verkregen in de Hoofdstukken 6, 7, 8 en 9. Eerst wordt ingegaan op de vraag of de realisaties van discrete emoties in verschillende culturen bepaalde kenmerken met elkaar gemeen hebben. Zowel de decriptieve gegevens als de resultaten van onze herkenningsexperimenten met Japanse en Taiwanese luisteraars wijzen inderdaad in die richting. Alleen voor blijdschap kunnen met grote zekerheid naast een aantal overeenkomsten essentiële maar nog niet gedefinieerde verschillen worden aangenomen. Wat de aard en de functie van emotionele dimensies betreft, bevestigen de gegevens met betrekking tot descriptie en herkenning elkaar eveneens: uit beide blijkt het belang van vocale parameters die verband houden met de activiteitsdimensie en de ongrijpbaarheid van de evaluatieve dimensie. Verder blijkt een discriminantanalyse gebaseerd op de perceptieve scores voor vijf vocale parameters tot een bijna even groot aantal correcte identificaties te leiden als met menselijke luisteraars wordt bereikt. Het aantal correcte identificaties op basis van akoestische informatie is aanzienlijk kleiner. Naast deze inhoudelijke conclusies worden er in dit hoofdstuk nog enkele meer methodologisch gerichte conclusies getrokken, en worden er suggesties gedaan voor verder onderzoek. 


\section{Curriculum vitae}

Renée van Bezooijen werd op 21 mei 1952 in Kortrijk (België) geboren. In 1970 slaagde zij voor het eindexamen Gymnasium $\alpha$ aan het Johan de Witt Gymnasium in Dordrecht. In hetzelfde jaar begon zij met de studie Franse Taal- en Letterkunde aan de Katholieke Universiteit van Nijmegen. In 1974 behaalde zij het kandidaatsexamen Frans en in 1977 het doctoraalexamen Algemene Taalwetenschap (cum laude). Daarna is zij gedurende een half jaar lerares Frans geweest aan het Gemeentelijk Lyceum in Zutphen.

Van december 1977 tot december 1979 was zij uitvoerster van een door de Nijmeegse onderzoekspool gefinancierd project getiteld "Problemen met betrekking tot het onderwijs en de toetsing van luistervaardigheid in het mavo-havo-vwo". Van december 1979 tot december 1982 was zij uitvoerster van het onderzoeksproject "Intonatie en emotie", waarover in dit proefschrift wordt gerapporteerd. Ook dit project werd door de Nijmeegse onderzoekspool gefinancierd. Sinds april 1983 is zij uitvoerster van een door ZWO gefinancierd project getiteld "Het relatieve belang van uitspraak, prosodie en stemkwaliteit voor de attributie van sociale status en persoonlijkheidskenmerken". 



\section{STELLINGEN}

1. Het uitvoeren van het in dit proefschrift beschreven onderzoek is met naar verhouding weinig emoties verlopen.

2. Als emoties in het dagelijks leven net zo duidelijk zouden worden gerealiseerd als in deze studie (zie proefschrift), zou het aantal communicatiestoornissen aanzienlijk worden teruggebracht.

3. Als reactie op de resultaten van een langdurig onderzoek is niets dodelijker dan "dat had ik je zo ook wel kunnen vertellen".

4. Ook begeleiders hebben soms begeleiding nodig.

5. Het toestaan van een grotere vrijheid in de spelling evenals het stilzetten van klokken bij opnames zou het sociolinguistisch onderzoek naar taalvariatie ten goede komen.

6. Het vinden van ambigue zinnen ten behoeve van wetenschappelijk onderzoek is moeilijker dan men op grond van hun frekwentie van voorkomen in het dagelijks leven zou verwachten.

7. Het is verrassend hoe goed proefpersonen in semantische-differentiaalexperimenten lijken door te hebben welke schaal op welke factor hoort te laden.

8. Het objectief meten van geluidsoverlast in $\mathrm{dB}$ zou moeten worden vervangen door een subjectieve maat in termen van eenheden irritatie.

9. In het leven wordt de overgang van "al" naar "nog" vaak als pijnlijk ervaren, terwijl het passeren van gestandaardiseerde overgangstijdstippen als het zien van Abraham feestelijk wordt gevierd.

Stellingen behorende bij:

Characteristics and recognizability of vocal expressions of emotion, R.A.M.G. van Bezooijen. 




\section{dé}

Printed by ICG Printing, Dordrecht 